Paradoxes of Interactivity / The Co-Evolution of Humans and Machines: A Paradox of Interactivity / I. Rethinking Interactivity / Does the Body Disappear? A Comment on Computer Generated Spaces / Transparency and Opacity: Interface Technology of Mediation in New Media Art / Where the Action is: Distributed Agency between Humans, Machines, and Programs / Surface, Interface, Subface: Three Cases of Interaction and One Concept / Double Cross Playing Diamonds: Understanding Interactivity in/between Bigraphs and Diamonds / II. Interplay between Art, Science, and Technology / Where Art and Science Meet (or Where They Work at Cross-Purposes) / Time, Magma, Continuity: Some Remarks on In-Formation and the Fabrication of "Poiēsis" / Implications of Unfolding / UNORTKATASTER: An Urban Experiment Towards Participatory Media Development / Modelling and Analysing Expressive Gesture in Multimodal Systems / III. Interactive Media Performances: Past, Present, and Future / Interaction Computer Dance: The Resonance Paradigm 1900/2000 / Staging of the Thinking Space: From Immersion to Performative Presence / From Interactive Live Electronic Music to New Media Art / Extending the Musical Experience: From the Physical to the Digital and Back / Virtual Musical Instruments and Robot Music Performances / Perspectives for Media Theory, Human-Computer Interaction, and Artistic Investigations

\title{
[transcript] Cultural and Media Studies
}


Uwe Seifert, Jin Hyun Kim, and Anthony Moore (eds.) PARADOXES OF INTERACTIVITY 

Uwe Seifert, Jin Hyun Kim, and Anthony Moore (eds.)

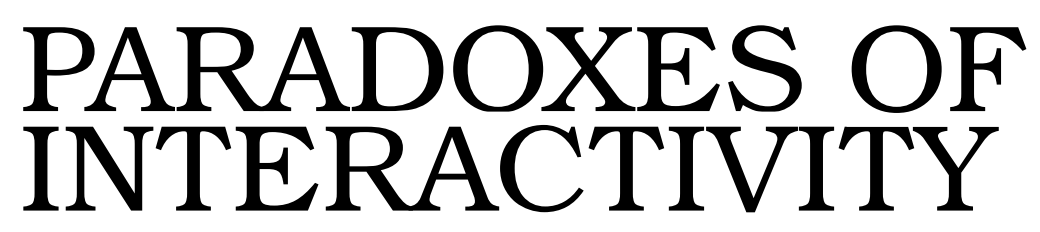

Perspectives for Media Theory,

Human-Computer Interaction, and Artistic Investigations

[transcript] 
This publication was financially supported by the German National Research Foundation (Deutsche Forschungsgemeinschaft) within the scope of the Collaborative Research Centre SFB/FK 427 "Media and Cultural Communication".

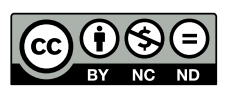

This work is licensed under a Creative Commons Attribution-NonCommercial-NoDerivatives 3.0 License.

\section{Bibliographic information published by the Deutsche Nationalbibliothek}

The Deutsche Nationalbibliothek lists this publication in the Deutsche Nationalbibliografie; detailed bibliographic data are available in the Internet at http://dnb.d-nb.de

\section{(C) 2008 transcript Verlag, Bielefeld}

Edited by: Uwe Seifert, Jin Hyun Kim, Anthony Moore Proofread by: Anthony Moore, Michael Kelly, Jin Hyun Kim, Jochen Arne Otto, Uwe Seifert

Typeset by: Jochen Arne Otto, Son-Hwa Chang Design: Carsten Goertz (www.farn.cc)

Layout: Jochen Arne Otto, Carsten Goertz, Rosie Placzek, Katja Schwemmer Printed by: Majuskel Medienproduktion $\mathrm{GmbH}$, Wetzlar ISBN 978-3-89942-842-1 



\section{CONTENTS}

Uwe Seifert

The Co-Evolution of Humans and Machines: A Paradox of Interactivity

\section{Rethinking Interactivity}

Sybille Krämer

Does the Body Disappear?

A Comment on Computer Generated Spaces

Ludwig Jäger and Jin Hyun Kim

Transparency and Opacity: Interface Technology

of Mediation in New Media Art

Werner Rammert

Where the Action is: Distributed Agency

between Humans, Machines, and Programs

Frieder Nake

Surface, Interface, Subface: Three Cases of Interaction

and One Concept

Rudolf Kaehr

Double Cross Playing Diamonds: Understanding Interactivity in/between Bigraphs and Diamonds

\section{Interplay between Art, Science, and Technology}

Hans H. Diebner

Where Art and Science Meet

(or Where They Work at Cross-Purposes) 
Time, Magma, Continuity: Some Remarks on In-Formation and the Fabrication of "Poiēsis"

Julian Rohrhuber

Implications of Unfolding

Georg Trogemann, Stefan Göllner, and Lasse Scherffig

UNORTKATASTER: An Urban Experiment Towards

Participatory Media Development

Antonio Camurri, Barbara Mazzarino, and Gualtiero Volpe

Modelling and Analysing Expressive Gesture

in Multimodal Systems

\section{Interactive Media Performances: Past, Present, and Future}

Martina Leeker

Interaction Computer Dance:

The Resonance Paradigm 1900/2000

Monika Fleischmann and Wolfgang Strauss

Staging of the Thinking Space:

From Immersion to Performative Presence

Jin Hyun Kim

From Interactive Live Electronic Music to New Media Art

Gil Weinberg

Extending the Musical Experience:

From the Physical to the Digital and Back

Suguru Goto

Virtual Musical Instruments and Robot Music Performances 


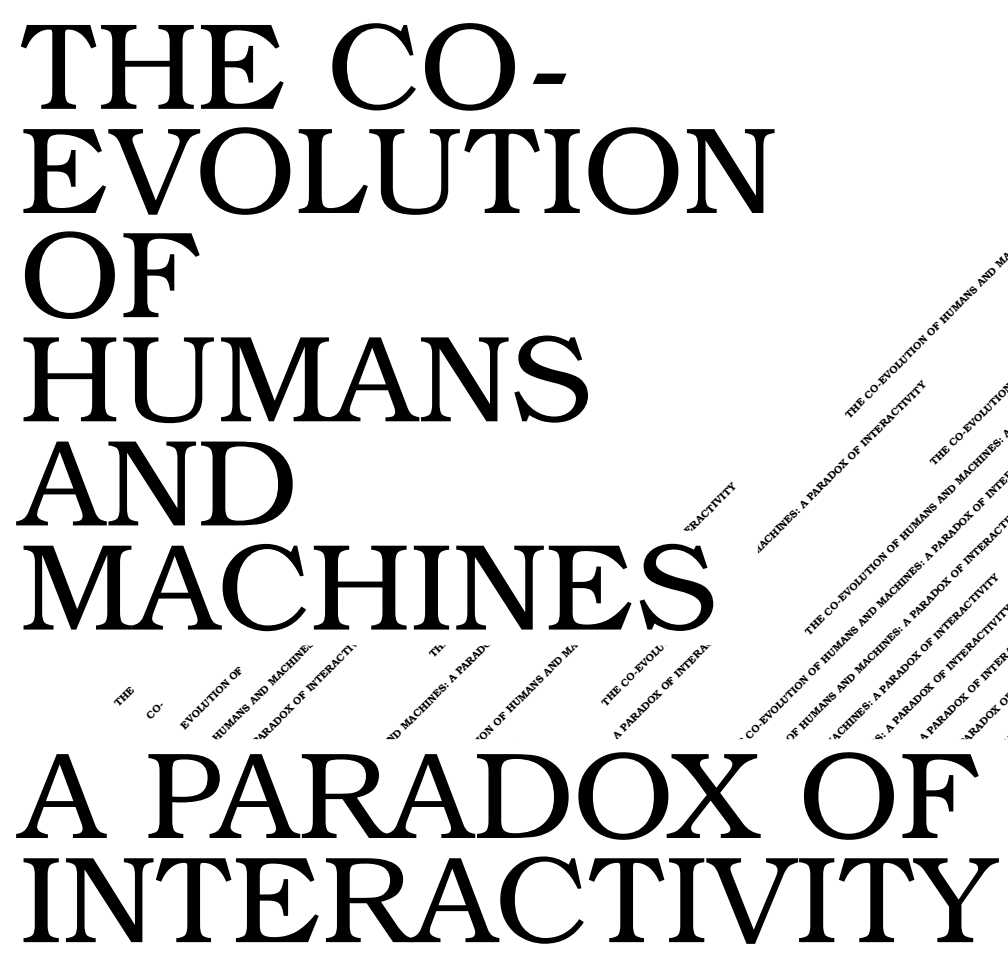

Uwe Seifert 


\section{The Meaning of "Paradox"}

What is meant by the expression "paradox" in "Paradoxes of Interactivity"? "Paradox" as used in the title of the book refers to the ordinary meaning of the word, and not to the well-known paradoxes of logic and mathematics such as Russell's ${ }^{1}$ set-theoretical paradox or Zeno's ${ }^{2}$ paradoxes of plurality and motion. The semantic field of the ordinary meaning of the word "paradox" derives from the ancient Greek word "parádoxos" consisting of "pará" meaning "contrary" and "dóxa" meaning "opinion". In Book V of his "Republic" Plato $^{3}$ speaks of "paradoxos logos". Used in this sense the meaning of "paradox" is "a statement contrary to expectation", "an incredible statement", "a statement contrary to accepted opinion", "against common sense or ordinary opinion", "provocative to accepted opinion or common sense", "contrary to generally accepted belief" or "something surprising". What are the provocative or incredible ideas associated with "interactivity"?

\section{Interactivity: A Semantic Field}

In general, an explanation of "interactivity" or "interaction" refers back to "action", and in the social sciences action is presupposed to depend on an active human subject intentionally acting upon an object or another subject. Interaction only takes place between humans, because objects, like machines, are incapable of intentionality. In the case of human action humans are ascribed agency. In sociology "agency" is often contrasted with "structure". But actually, structure is both an outcome of previous agency and a constraint upon it. Two semantic fields can be associated with "agency". First, in the social sciences the sense of "agency" is mainly judicial, political or economical. This meaning is related to authority and assignment of power or official duties to humans, e.g. "assignee" or "agent". The second meaning of "agency" and "agent" is mostly found in the natural sciences such as chemistry, biology, and physics. It is associated with effect, tool, activity, e.g. "protective agent". To summarise: There are two fields of meanings concerning "agency", "agent", "action", "interaction" and "to act upon". One semantic field concerns the social sciences, and strongly relates to the idea of an intentional being, a human. The other concerns the natural sciences and refers to the idea of effect.

Russell 1903, \& 101

Salmon 2001

3 Plato's "Republic" 472 a6; see Liddell/Scott 1996, p. 1309 
With the advent of computational technology and systems the situation has changed: machines are attributed the active role in human's use of machines. They become subjects of actions and agents. One speaks of humans interacting with computers.

Agent technology and social robotics are two important recent examples to illustrate that the difference between humans and machines is becoming increasingly blurred. The meaning of "agent" applied in the social sciences only to human agents is being transferred to software: Special tasks such as internet searches and communication on behalf of a human are assigned to personal software agents. ${ }^{4}$ In entertainment, therapy, e.g. autism therapy, and at home, e.g. as a robot companion, robots are interacting with humans. Furthermore, ubiquitous computing seems to make the computer "disappear" and at the same time be more and more entangled in day-to-day life: Things are beginning to talk. ${ }^{5}$

Furthermore, the asymmetric relation of human's use of machines is becoming symmetric. Machines are becoming the subject of interaction. Humans are interacting with machines, machines are interacting with machines and humans are interacting with humans via machines. In general, there are hybrid networks consisting of human and machine interacting with each other. The use of "interactivity" suggests that the difference between humans and machines evaporates. This is the main paradox associated with "interactivity"!

\section{Humans and Machines: An Evolving Discontinuity}

"Interactivity" indicates that at present a phase of fundamental change is being undergone in the ontological difference between humans and machines: This discontinuity is beginning to disappear. This important insight has been realised but articulated differently by many authors from different disciplines. ${ }^{6}$ Especially Bruce Mazlish developed this insight further and pointed out that in order to cope with emerging social and cultural problems humans must accept the continuity of humans and machines. His thesis is that "man is breaking past the discontinuity between himself and machines." Mazlish argues: Man is now becoming aware that "his own evolution is inextricably

\footnotetext{
4 Payr/Trappl 2004; Dautenhahn 2002

5 For instane, O'Sullivan/Igoe 2004 and Igoe 2007 introduce into physical computing in media art and design using Arduino, Processing and other "tools".

6 E.g. Robertson 1998, 2003; Ford/Glymour/Hayes 2006; Hubig/Koslowski 2008; Mazlish 1967, 1993

$7 \quad$ Mazlish 1967, p. 14; Mazlish 1993 elaborates the fourth discontinuity thesis.
} 
interwoven with his use and development of tools" , and that "the same scientific concepts help explain the workings of himself and of his machines."

At present the strongest scientific thesis in this sense is put forward by cognitive scientists: They argue that human beings are (logical) automata or that all natural human functions are best explained by (finite) automata in the sense of automata theory. ${ }^{10}$

Mazlish's claim is strongly supported by the emergence of new scientific disciplines, subdisciplines and research areas as well as art forms and cultural applications of computing, such as, to mention just a few, cognitive science, computational and cognitive neuroscience, techno- and biosciences, ubiquitous, physical and art computing, social and educational robotics, neuro-robotics, human-computer and human-robot interaction, interaction design, and interactive and new media art.

\section{"Action" and "Interaction": Some Definitions}

These developments require the social sciences, especially sociology, to interpret "interaction", "interactivity", "agency" and "agent" in the same manner as the natural sciences. Mario Bunge developed definitions of these terms whose meanings encompass both the social and the natural sciences. ${ }^{11}$

He considers "action" as a general (ontological) concept. The general idea captured in formalising the action relation is "What one thing does to another." ${ }^{12}$ In his formalisation ${ }^{13}$ Bunge uses concepts from set theory in order to define the action relation " $\mathrm{x}$ acts upon $\mathrm{y}$ " or "the action that thing $\mathrm{x}$ exerts on thing $y$ ". The expression " $x$ acts upon $y$ " is defined as a set-theoretic difference or relative complement of the history of $y$ in the presence of $x$, and the history of $\mathrm{y}$ in the absence of $\mathrm{x}$. The history of an object $\mathrm{x}$ is formed by the values $v$ of its state function $F$ for all time points $t$ over a time period $T$. A state function $F$ can be conceived of as a list of all known properties for some kind of objects. The concept of "interaction" is based on the definition of "to act upon": Two different things interact if and only if each acts upon the other. Human action appears as a special case of action. An action is a human action if and only if at least the agent of the action relation is a per-

\footnotetext{
$8 \quad$ Mazlish 1967, p. 14

$9 \quad$ Mazlish 1967, p. 14

10 E.g. Boden 2007; Burks 1972-73, 1990; Nelson 1988. Concerning the terms "machine" and "finite automata" see Minsky 1972, pp. 1-7 and pp. 11-31.
}

11 Bunge 1998, 2003

12 Bunge 2003, p. 9

13 Rudolf Kaehr's contribution takes a formalised approach to interaction. Kaehr's contribution should be viewed in the context of Gotthard Günther's ideas concerning the formalisation of the ideas of an "objective spirit/mind" and dialectic logic. 
son. A social action is an action, in which both relata, agent and patient, are persons or one of them is a social system or public good. Agent and patient are defined as relata of the action relation. In an action relation " $x$ acts upon $y$ " the relatum $\mathrm{x}$ is called "the agent" and $\mathrm{y}$ "the patient", if $\mathrm{x}$ acts upon $\mathrm{y}$. Both entities are said to "interact" in case the patient $y$ reacts back on the agent $x$ that initiated the process, i.e., $\mathrm{y}$ acts upon $\mathrm{x}$, and $\mathrm{y}$ becomes the agent and $\mathrm{x}$ the patient. In such a case, except for practical purposes, the agent/patient distinction disappears. Based on these definitions, Bunge defines several other concepts associated with "action" such as "consequence of an action" and "reaction".

As exemplified by Mario Bunge's definition of terms such as "action" and "interaction", the meanings of "action" and "interaction" encompass human and non-human actions. Human actions and interactions form a special case of the broader definition of "action" and "interaction" and, in general, for the relata of interactions no distinction is made concerning agenthood and patienthood.

\section{Interactivity and Interaction as Symmetrical Relations}

Even if it is not necessary for action and agency to be associated with humans, as shown by the definitions given by Mario Bunge, it can be seen in sociology and philosophy of technology that these terms are often only ascribed to humans and not to machines. For example, in the German synthetic or pragmatic philosophy of technology developed by Hans Lenk and Jürgen Ropohl, humans interacting with machines in order to achieve a goal are considered to form an integral system, a socio-technological action unit. ${ }^{14}$ In this view, even though a machine and a human form an integral action unit, the machine only concurs to the human action. A machine is not considered as an agent or actor, because it lacks intentionality and (human) purpose. This restriction implies that the action relation is thought to be asymmetric concerning the kinds of relata. Only a special kind of relata can be agents. Taking into account particularly recent directions of research into human-computer interaction (HCI) and human-robot interaction (HRI), concepts or theories that assign activity only to humans and passivity to machines seem dubious.

Bruno Latour's actor-network theory and socionics, ${ }^{15}$ an approach to sociology which combines computer science and sociology, are in contrast

14 The German term is "soziotechnisches System".

15 Werner Rammert's contribution addresses some implications of socionics for interaction. 
to Hans Lenk's and Jürgen Ropohl's synthetic and pragmatic philosophy of technology. ${ }^{16}$ These theoretical approaches propose considering the action relation between humans and machines to be symmetrical, and advocate a kind of anthropology, especially a symmetrical anthropology, which views the roles of machines in human-machine interaction in general to be equated with human roles.

The insight into the viewing of human-machine systems as integrated systems has been made by Arthur W. Burks for computers in connection with the most effective use of both humans and computers. He uses the term "human-computer combines" 17 and points out the importance of the social implications of their use: "Electronic computers are the first active or "live" mathematical systems. ... The most effective use of computer programs is to instruct computers in tasks for which they are superior to humans. Computers are being designed and programmed to cooperate with humans so that the calculation, storage, and judgment capabilities of the two are synthesized. The powers of such human-computer combines will increase at an exponential rate as computers become faster, more powerful, and easier to use, while at the same time becoming smaller and cheaper. ${ }^{18}$ The social implications of this are very important."

So far, our discussion of human-machine interaction has revealed two important aspects of the human-machine relationship: 1) In general, there is no logical necessity to associate "action", "interaction", and "interactivity" only with humans, especially not in the case of human-computer and human-robot interaction. 2) Furthermore, human-machine systems form an integrated system that increases the power of both human and machines.

Concerning the goals of artificial intelligence, the idea of thinking machines and the discussions about human and machine intelligence, Harel makes the following distinction: "Perhaps, instead of AI, "artificial intelligence", the emphasis should be on IA, intelligence augmentation, which is the development of computerized tools that enhance human intelligence and improve its functioning. Combining the best aspects of human and machine may bring about that neither can do in its own." 19

Burks describes this situation for scientific research on goal-directed and intentional systems for developing robots: "The ways in which models are used by goal-directed systems to solve problems and adapt to their environment are currently being modelled by human-computer combines. Since

16 See Maring 2008, p. 118.

17 Burks 1999, p. 167

18 This indicates the relevance of the human-in-the-loop.

19 Harel 2004, p. 400. Technologically speaking, at present most "interactive" systems for social or artistic interactions are reactive systems. See Harel 2004 for more information on reactive systems. 
computer software can be converted into hardware, successful simulations of adaptive uses of models could be incorporated into the design of a robot. Human intentionality involves the use of model of oneself in relation to other and the environment. A problem-solving robot using such a model would constitute an important step toward a robot with full human powers." ${ }^{20}$

Science, especially cognitive science and research on human-computer and human-robot interaction, uses interactive art as a test bed in order to study action, perception, and cognition. This idea is elaborated in the next paragraph.

\section{Interactivity and Cognition: Environments, Affordances, and Effectivities}

It is important to note that the internal model of oneself is used in relation to others and not only to an environment. Furthermore, it must be pointed out that human interaction and communication takes place in a social and cultural environment rather than in a biological or physical environment. A social or cultural environment differs in many respects from a natural environment. The most important difference seems to be the use of symbolisation in social and cultural interaction and communication.

An agent's situatedness and "interactions" with an environment are highly important for the study of cognitive and perceptual capacities. In the context of these studies the concept of "affordances" is essential. The idea of "affordances" plays an important role both in the study of human-computer interaction ${ }^{21}$ and interaction design and in neuro-robotics and cognitive and behavioural robotics. Gibson introduced the term "affordance" to the psychology of perception, and Norman to human-computer interaction and interaction design. For Gibson affordances are action possibilities available in an environment, independent of an agent's ability to perceive these possibilities. These are the actual possibilities of the environment. Affordances are conceived of as information in the sensory stream concerning opportuni-

\footnotetext{
20 Burks 1999, p. 168
}

21 The use of methods from human-computer interaction in non-technological context in combining HCI research and interactive art is a new emerging field, e.g. Höök/ Sengers/Andersson 2003. At first glance there seems to be a difference between actions in everyday life, at work and art. It is possible to think of a work of art as consisting of interacting objects that humans are passively experiencing. In such a case humans are patients, and the artwork is the agent acting upon humans. Another more general scenario is that humans play an essential role in interactive art and are acting upon the objects and machines: This is the human-in-the-loop. In interactive art the human is necessary for interaction. 
ties for action in and provided by the environment. Norman uses the term "affordances" for perceived possibilities, even if they may not actually exist. ${ }^{22}$

"Effectivities" is the concept complementary to "affordances". They "are the range of possible deployments of the organism's degrees of freedom," and "... the development of novel effectivities creates opportunities for the recognition of new affordances, and vice versa." ${ }^{23}$

In general, affordances provide cues to the operation of objects. They are the link between tools or objects and the knowledge of their use, i.e. the operational chains.

Effectivities expand the range of affordances. Gibson's and Norman's concepts of "affordances" neglect affordances in the case of social interaction, i.e. where the tools or objects are robots or humans. It might be a good idea to expand the ideas of "affordances" and "effectivities" to the study of social human-machine interaction, especially human-robot interaction and media art. To what extent are the actions of others in social interaction guided by affordances and effectivities? What the effectivities and affordances in social and emotional interactions with machines are remains an open question for research.

As noted previously, new media art and interaction art are increasingly being used as a test bed for scientific research. In interactive and media art human-computer combines can be used to enhance artistic productivity: artistic human-computer combines may form expression and structures that humans or computers alone can't achieve. At the same time, in applying theories and methods provided by the sciences, art explores the capacities of humans to sense, perceive, and act in unknown environments: Scientists become artists and artists become scientists. Therefore, in using humancomputer combines and robots in science and art, it seems that the boundary between science and art is increasingly being blurred. One claim concerning this development is that art and science - as in the Renaissance ${ }^{24}$ - are beginning to form a new alliance. ${ }^{25}$ The artistic use of current developments in robotics, ${ }^{26}$ artificial life, ${ }^{27}$ software algorithms, ${ }^{28}$ and agents ${ }^{29}$ as well as

22 For a detailed analysis of different theoretical uses of "affordance" in Gibson 1979 and Norman 1988 see Gaver 1991 and McGrenere/Ho 2000.

23 Arbib 2006, p. 6

24 See Douglas Robertson's analysis for science and everyday life.

25 Hans Diebner's contribution elaborates this idea.

26 On the artistic use of robots see the contributions by Suguru Goto; Gil Weinberg.

27 See the contributions by Jin Hyun Kim; Christoph Lischka.

28 See the contributions by Frieder Nake; Julian Rohrhuber.

29 See the contribution by Georg Trogemann, Stefan Göllner, and Lasse Scherffig. 
mixed, augmented and virtual reality ${ }^{30}$ makes the relocation of the relationship between humans and artefacts evident.

New Media Art is seen in this context as a field in which art, science and technology are interwoven. As media artists are not only involved in artistic practices, but also in investigations analogous to science, ${ }^{31}$ (artistic) creativity is needed in science to deal with new epistemological problems which come to focus through newer technologies. ${ }^{32}$ Technologies are not only a means to achieve a goal, but rather a mediator for artistic and scientific experiments, which serves as component of efficiency respectively effectiveness. ${ }^{33}$

\section{Interactivity and Media Theory}

The transformation of technical tools, which began with the advent of digital technologies and led to the so-called New Media, changes our habitual modes of media use, reshapes our experience mediated by media, and opens up the possibilities of new designs of artistic and scientific experiments. Especially, a Media Theory concerning New Media, and particularly New Media Art, needs an alternative view to traditional conceptualisations of agency and interactivity, within a conceptual framework in which humanmachine interaction can be based on an asymmetric relation and a co-active taking of effect during this interaction can be seriously investigated. In this context, rethinking "interactivity" opens a perspective for media theory from the point of view of cultural science and humanities which directs a research focus towards different themes related to New Media.

In Germany, there has been a paradigm shift within the humanities so that different conceptions of "Medien" (media) have come to the fore since the 1990s as a paradigm in contrast to "Geist" (spirit) and "Kultur" (culture). Contrary to media computing or psychology, media theory, oriented towards cultural science, surveys the operations of media which form and constitute the mediatised. Media act as preconditions for cultural semantics and psychological experiences. Research focuses were traditionally directed towards technical apparatuses or symbolic means, assuming some kind of "pure" meaning or intentional communication. Newer approaches of media theory do not assume that there is media-free and pre-medial meaning, information or intention that can be conveyed by media. Rather, media not only

30 See the contributions by Antonio Camurri, Barbara Mazzarino, and Gualtiero Volpe; Monika Fleischmann and Wolfgang Strauss; Ludwig Jäger and Jin Hyun Kim; Sybille Krämer; Martina Leeker.

31 See the contributions by Hans Diebner; Julian Rohrhuber.

32 See the contributions by Hans Diebner; Christoph Lischka.

33 See the contributions by Werner Rammert; Julian Rohrhuber. 
act as an indifferent means of conveying the mediatised, but also participate in its shaping. ${ }^{34}$ Therefore, "mediality" as the main operation of media which refers to the relation of a medium to the mediatised comes to the fore. The traditional concept of interactivity is not commensurable with the basic idea underlying this paradigm for media research within cultural science.

The main research interest of media theory related to interactive media is the question of how different media formats may have an effect on the meaning formation, information or experience generated co-actively by interactants. ${ }^{35}$ For instance, how the practices of virtuality offer the possibility of interactivity with symbol structures, makes clear the difference of computer-based media from literal media. ${ }^{36}$ Information technological and artistic experiments with HCI and HRI can therefore be investigated in respect of medial operations of newer technologies ${ }^{37}$ which can not only serve as an analysis of mediality of New Media, but also have an impact on information technological research on HCI and HRI and artistic practices.

\section{Interactivity and Emotion: Relational Artefacts}

So far, interactivity and interaction have been dealt with in connection with the logical problem of defining "action" and "interaction" and discussions in the philosophy and sociology of technology concerning its relational property as being either symmetric or asymmetric. Furthermore, the importance of considering human-machine combines as integrated systems and, especially for human-computer interaction, as augmenting the power of both humans and computers for scientific research and artistic projects was mentioned. The convergence of art and science in using interactive art as a test bed for testing scientific hypotheses was noted. The importance of distinguishing between a natural and a social or cultural environment was pointed out. This distinction is based on symbolisation for communication, information exchange and transmission in cultural or social environments. Media theory was introduced as a point of view from the humanities concerned with the role of media in symbolisation and meaning formation. However, so far, the importance of emotional and social interaction between humans and machines has been neglected.

In the epilogue of the 2004 twentieth-anniversary edition of her famous "The Second Self: Computers and the Human Spirit" from 1984 the psychoanalyst Sherry Turkle reflects on the present situation concerning the rela-

34 Tholen 2005, p. 166

35 See Martina Leeker's contribution.

36 See Sybille Krämer's contribution.

37 See the contribution by Ludwig Jäger and Jin Hyun Kim. 
tion between humans and machines. For her the main question is not about the real emotional and intellectual capacities of machines, but rather about human vulnerability and the human self-image. In analysing the present human-machine relationship and the current technological and social developments, she coined the term "relational artifacts" for artefacts that "ask their users to see them not as tools but as companions, as subjects in their own right" 38 that "... present themselves as sentient and feeling creatures, ready for relationship." 39 For Sherry Turkle "The new questions are not about whether relational artifacts will really have intelligence and emotions but about what they evoke in their users." ${ }^{40}$ The question concerns "..., what we will be like"41 and not what computers or robots can do. Furthermore, she points out that social and emotional interaction with machine is no longer science fiction, but rather social reality, and may affect the way humans think about themselves and their social relations: "The introduction of robotic helpers in nursing homes, ..., is now being presented in the United States as potential social policy. $[. .$.$] How will interacting with relational artifacts affect people's way of$ thinking about what, if anything, makes people special? The sight of children and the elderly exchanging tenderness with robotic pets brings science fiction into everyday life and technophilosophy down to earth. The question is not whether children will love their pet robots more than their real life pets or even their parents, ${ }^{42}$ but rather, what will loving come to mean." ${ }^{33}$

The last paragraphs show that, in interacting, human and computers form an integrated whole, and it seems appropriate to conceptualise the interaction of humans and computers as a symmetric relation. Computers are not only conceived of as tools; rather they are best conceptualised as "partners", because computers and robots are increasingly, just like humans, acting in social and cultural environments. Symbolisation was identified as important in order to distinguish interaction with natural environments from interaction in social or cultural environments. As a final step the current developments in technology and interaction are put into a historical and evolutionary perspective of humankind.

\footnotetext{
38 Turkle 2005, p. 289

39 Turkle 2005, p. 288
}

40 Turkle 2005, p. 294; Fellous / Arbib 2005 contains further information of the current state of the art in research on the human brain, emotions, and robots. Social humanmachine interaction based on detection and emulation of emotional states is treated e.g. in Picard 2002 and Breazeal 2002. Dautenhahn 2002, 2007 and Dautenhahn et al. 2002 treat social aspects of human-machine interaction in general.

41 Turkle 2005, p. 294

42 One may speculate that children will like their robot teachers more than their human teachers. For more information on learning, children, and robots, see Druin/ Hendler 2000.

43 Turkle 2005, pp. 295 


\section{Human-Machine Interaction: A Broader View}

A perspective which is not dealt with in this book but which underlies its conception is that tool use, symbolisation, and the evolution of the human mind are interwoven. ${ }^{44}$ It is hypothesised that humankind is in a new phase of its cultural evolution which started with tool use and language 100 thousand years BP, continued with the invention of script 4000 years BC, and printing in $1500 \mathrm{AD}$ and digital technology in the 20th century.

In Anthropology and prehistory the speciation of humankind has been associated with tool use, bipedality, increasing brain size and lateralisation, and symbolisation, to mention just a few of the proposed characteristics that distinguish humans from monkeys and apes. But now there is increasing evidence from primatology, anthropology and prehistory that social intelligence, interaction, and communication seem to be the causes of the differences that distinguish the hominin line from the other hominids. Since the advent of the human species, biological evolution has become more and more a cultural "evolution" in connection with symbolisation ${ }^{45}$ and the tools for communication, information exchange and cultural transmission. The invention of writing systems and the printing press are well-known examples. But one has to bear in mind that these tools serve to facilitate social purposes such as cultural communication, information transmission and exchange.

The important point to note is that digital information technology operates in social realms of interaction, intelligence, and communication. Furthermore, it is important to note that it is not the material culture, i.e. the physical objects, in itself that is of importance, but rather the procedural knowledge associated with their use: the techniques or (procedural) knowledge of their use. The ethnologist Edwin Hutchins uses the term "cognitive artifact" as a concept which "... points not so much to a category of objects, as to a category of processes that produce cognitive effects by bringing functional skills into coordination with various kinds of structures." ${ }^{46}$ Similarly in relation to tools and their use the French archaeologist Leroi-Gourhan pointed out the relevance of operational chains, i.e. operational sequences of technical actions. ${ }^{47}$

This may raise the question: What are the operational chains or cognitive artefacts which guide social interactions with machines, especially in

44 On human evolution, technology, and cognition see e.g. Audouze 1999; Gibson/ Ingold 1993; Washburn 1960.

45 Donald 1991, 2001; Lock/Peters 1996

46 Hutchins 1999, p. 127. There seems to be a close connection to some uses of "media" and the idea of mediality in media theory. Unfortunately this relation cannot be elaborated here.

47 Leroi-Gourhan 1964; the French term is "chaînes opératoires". 
an artistic context, and how do they change and develop in social humancomputer and human-robot interaction? In general, how are they identified? All these questions are open for further research.

\section{Conclusion}

Currently we are at a crossroad in the co-evolution of humans and machines. We have identified symbolisation or symbolic communication and social interaction as the core of this co-evolution. It is claimed that the arts, especially New Media Art, in connection with physical computing, social robotics, and human-robot interaction, are becoming an extended "laboratory" for scientific research on social interaction and the human mind and its underlying psychological and neuronal mechanisms, as well as the cultural origins of higher cognitive functions. At the same time they are exploring new effectivities and affordances in the social or cultural art environments. Furthermore, interactivity in human-machine interaction is no longer merely a technological issue, or one only for scientists and engineers. Neither is interaction a topic only for psychologists and sociologists. The effects and consequences of human-computer and human-robot interaction are becoming an issue concerning all aspects of social human life and existence. Especially concerning the design of robots and human-robot interaction, ethical topics must be urgently addressed. Because of its impact on social communication and structure and the importance for the human self-image, new conceptualisations for describing, analysing, and theory-forming, as well as empirical research methods, are urgently needed to study this development. Media art in connection with cognitive and media science, human-computer and human-robot interaction is one of the best ways to cope with this need. It is not false to predict that in the near future the importance of its social, educational, political, philosophical, and theological implication will become tremendous. For example, Sherry Turkle observes: "Both psychoanalysis and computation challenge common sense understandings of action and responsibility because they get people thinking of a 'decentered' self - a self that is not a unitary, intentional agent." ${ }^{8}$ The co-evolution of humans and machines will have essential effects on the human mind. Therefore, it is important to bear in mind Merlin Donald's assertion: “... the role of the individual mind is changing, not in trivial ways but in its essence. And these changes need watching." 49

\footnotetext{
48 Turkle 2005, p. 356

49 Donald 1991, p. 360
}

\section{0}


This book gives a bird's-eye view on the current situation and some hints on what to look for in order to watch. ${ }^{50}$

\section{References}

Arbib, Michael A. (2006): »The Mirror System Hypothesis on the Linkage of Action and Languages«. In: Michael A. Arbib (Ed.), Action to Language via the Mirror Neuron System, Cambridge: Cambridge University Press, 3-47.

Audouze, Françoise (1999): »Technology and Human Evolution«. In: Robert Wilson/ Frank C. Keil (Eds.), The MIT Encyclopedia of the Cognitive Sciences, Cambridge, MA: MIT Press, 828-829.

Boden, Margaret (2006): Mind as Machine: A History of Cognitive Science, Vol. 1/Vol. 2, Oxford: Clarendon.

Breazeal, Cynthia (2002): Designing Sociable Robots, Cambridge, MA: MIT Press

Bunge, Mario (2003): Philosophical Dictionary: Enlarged Edition, Amherst, NY: Prometheus Books.

Bunge, Mario (1998): Social Science Under Debate: A Philosophical Perspective, Toronto: University of Toronto Press.

Burks, Arthur W. (1999): „Computer Theory«. In: Robert Audi (Ed.), The Cambridge Dictionary of Philosophy, $2^{\text {nd }}$ Edition, Cambridge: Cambridge University Press, 164-168.

Burks, Arthur W. (1990): »The Philosophy of Logical Mechanism«. In: Merrilee H. Salmon (Ed.), The Philosophy of Logical Mechanism: Essay in the Honor of Arthur W. Burks, Dordrecht: Springer, 349-531.

Burks, Arthur W. (1972-73): »Logic, Computers, and Men«. In: Proceedings and Addresses of the American Philosophical Association 46, 39-57.

Clark, Andy (2003): Natural-Born Cyborgs: Minds, Technologies, and the Future of Human Intelligence, Oxford: Oxford University Press.

Dautenhahn, Kerstin (Ed.) (2002): Human Cognition and Social Agent Technology, Amsterdam: Benjamins Publishing.

Dautenhahn, Kerstin/Bond, Alan H./Cañamero, Lola/Edmonds, Bruce (Eds.) (2002): Socially Intelligent Agents: Creating Relationships with Computers and Robots, Dordrecht: Kluwer.

Dautenhahn, Kerstin (2007): »Socially Intelligent Robots: Dimensions of Human-Robot Interaction«, Philosophical Transactions of the Royal Society B 362, 679-704.

50 This book is in close relation to Sommerer/Mignonneau/King 2008 Interface Cultures: Artistic Aspects of Interaction, Lischka/Sicks 2007 Machines as Agency: Artistic Perspectives, Hubig/Koslowski 2008 Maschinen, die unsere Brüder werden, Svanæs 2000 Unterstanding Interactivity, Ford/Glymour/Hayes 2006 Thinking about Android Epistemology, and Clark 2003 Natural-Born Cyborgs. 
Donald, Merlin (2001): A Mind so Rare: The Evolution of Human Consciousness, New York: Norton.

Donald, Merlin (1991): Origins of the Modern Mind: Three Cultures in the Evolution of Culture and Cognition, Cambridge, MA: Harvard University Press.

Druin, Allison/Hendler, James (Eds.) (2000): Robots for Kids: Exploring New Technologies for Learning, San Francisco: Kaufmann.

Fellous, Jean-Marc/Arbib, Michael A. (Eds.) (2005). Who Needs Emotions? The Brain Meets the Robot, Oxford: Oxford University Press.

Ford, Kenneth M./Glymour, Clark/Hayes, Patrick (Eds.) (2006): Thinking About Android Epistemology, Cambridge, MA: MIT Press.

Gaver, William W. (1991): »Technological Affordances«. In: Scott P. Robertson/Gary M.

Olson/Judith S. Olson (Eds.), Proceedings of the ACM CHI 91 Human Factors in Computing Systems Conference, New Orleans, 79-84.

Gibson, James J. (1979): The Ecological Approach to Visual Perception, New Jersey: Erlbaum.

Gibson, Kathleen R./Ingold, Tim (Eds.) (1993): Tools, Language, and Cognition in Human Evolution, Cambridge: Cambridge University Press.

Harel, David (2004): Algorithmics: The Spirit of Computing. $3^{\text {rd }}$ Edition with Yishai Feldman, Essex: Pearsons Education Limited.

Höök, Kristina/Sengers, Phoebe/Andersson, Gerd (2003): »Sense and Sensibility: Evaluation and Interactive Art«. In: Proceedings of Computer-Human Interaction, April 5-10, 2003, Ft. Lauderdale, Florida, USA, Vol. 5(2), 241-248.

Hubig, Christoph/Koslowski, Peter (Eds.) (2008): Maschinen, die unsere Brüder werden - Mensch-Maschine-Interaktion in hybriden Systemen, München: Fink.

Hutchins, Edwin (1999): »Cognitive Artifact«. In: Robert Wilson/Frank C. Keil (Eds.), The MIT Encyclopedia of the Cognitive Sciences, Cambridge, MA: MIT Press, 126-127.

Igoe, Tom (2007): Making Things Talk: Projects and Ideas to Create Talking Thing Objects from Anything, Beijing: O’Reilly.

Leroi-Gourhan, André (1964): Le Geste et la Parole, vol. 1: Technique et Langage; vol.2 La Mémoire et les Rhythmes, Paris: Albin Michel.

Liddell, Henry George/Scott, Robert (1996): A Greek-English Lexicon: With a Revised Supplement 1996, Oxford: Clarendon.

Lischka, Christoph/Sicks, Andrea (Eds.) (2007): Machines as Agency: Artistic Perspectives, Bielefeld: transcript.

Lock, Andrew/Peters, Charles R. (Eds.) (1996): Human Symbolic Evolution, Oxford: Oxford University Press.

Maring, Matthias (2008): »Mensch-Maschine-Interaktion. Steuerbarkeit Verantwortbarkeit«. In: Christoph Hubig/Peter Koslowski (Eds.), Maschinen, die unsere Brüder werden - Mensch-Maschine-Interaktion in hybriden Systemen, München: Fink, 113-128.

Mazlish, Bruce (1993): The Fourth Discontinuity: The Co-Evolution of Humans and Machines, New Haven: Yale University Press. 
Mazlish, Bruce (1967): »The Fourth Discontinuity«. Technology and Culture 8(1), 1-15.

McGrenere, Joanna/Ho, Wayne (2000): »Affordances: Clarifying and Evolving a Concept«. In: Proceedings of Graphics Interface 2000 May 15-17, Montréal, Quebec, Canada, 38-41.

Minsky, Marvin (1972): Computation: Finite and Infinite Machines, London: PrenticeHall.

Norman, Donald (1988): The Psychology of Everyday Things, New York: Basic Books.

O’Sullivan, Dan/Igoe, Tom (2004). Physical Computing: Sensing and Controlling the Physical World with Computers, Boston, MA: Course Technology.

Platon (2005): »Der Staat«. In: Günther Eigler (Ed.), Platon - Werke in acht Bänden. Griechisch und Deutsch. Vierter Band: Platon - Der Staat. Bearbeitet von Dietrich Kurz. Griechischer Text von Èmile Chambry. Deutsche Übersetzung von Friedrich Schleiermacher. $4^{\text {th }}$ edition, Darmstadt: Wissenschaftliche Buchgesellschaft.

Payr, Sabine/Trappl, Hubert (Eds.) (2004): Agent Culture: Human-Agent Interaction in a Multi-Cultural World, London: Routledge.

Picard, Rosalind (1997): Affective Computing, Cambridge, MA: MIT Press.

Robertson, Douglas S. (1998): The New Renaissance: Computers and the Next Level of Civilization, New York: Oxford University Press.

Robertson, Douglas S. (2003): The Computer Revolution in Science and Mathematics, New York: Oxford University Press.

Russell, Bertrand (1903/ $\left.{ }^{2} 1996\right)$ : The Principles of Mathematics. Reprint of the 1938 $2^{\text {nd }}$ Edition, New York: Norton. Incomplete Online Edition: <http: / fair-use.org/bertrand-russell/the-principles-of-mathematics/index> (last access: March 2008).

Salmon, Wesley C. (Ed.) (2001): Zeno's Paradoxes, Indianapolis, Cambridge: Hackett Publishing.

Sommerer, Christa/Mignonneau, Laurent/King, Dorothée (Eds.) (2008): Interface Cultures: Artistic Aspects of Interaction, Bielefeld: transcript.

Svanæs, Dag (2000): Understanding Interactivity: Steps to a Phenomenology of HumanComputer Interaction, Trondheim: Norwegian University of Science and Technology (NTNU). Online available: <http://www.idi.ntnu.no/ dags/interactivity.pdf> (last access: March 2008).

Tholen, Georg Christoph (2005):»Medium/Medien«. In: Alexander Roesler / Bernd Stiegler (Eds.), Grundbegriffe der Medientheorie, Paderborn: Wilhelm Fink, 150-172.

Turkle, Sherry (2005): The Second Self: Computers and the Human Spirit. Twentieth Anniversary Edition, Cambridge, MA: MIT Press.

Washburn, Sherwood L. (1960): »Tools and Human Evolution«. Scientific American 204(6), 62-71. 


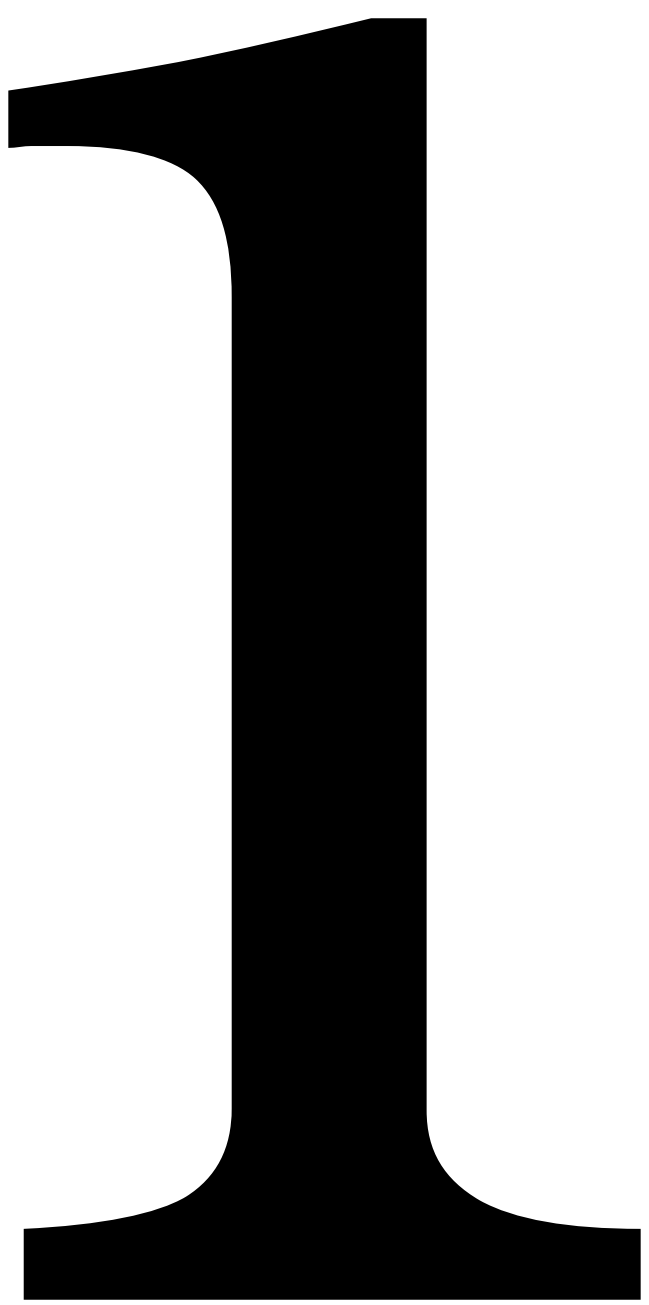




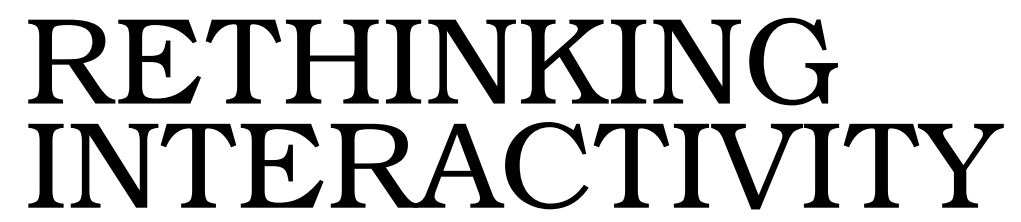




\section{DOES}

BODY

DISAPPEAR?

A

COMMENT

$\mathrm{ON}$

COMPUTER

GENERATED

SPACES
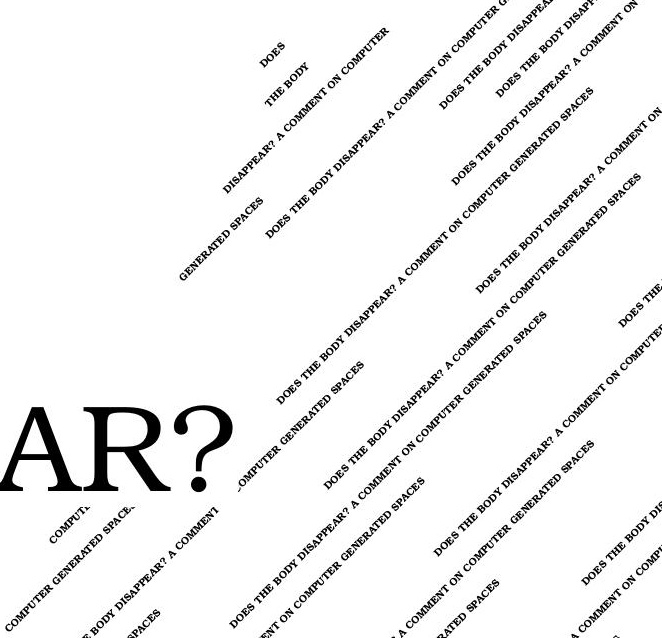


\section{Dematerialisation?}

The notion that the use of new media effects a dematerialisation constitutes a kind of lowest common denominator for the various designs of knowledge societies' perspectives: Norbert Wiener's distinction of information from matter and energy turned information into a quantifiable 'universal coin' that permitted all phenomena to be commensurable and transferable under the aspect of their telecommunicational coding, regardless of their spatiotemporal situatedness and their respective meaning. Marshall McLuhan's 'global village' proceeds from the assumption of an electronic shrinkage of spatial distance which enables the realisation of a form of telepresence that supersedes the principle of locality as a sine qua non of interactive communication. ${ }^{1}$ Hans Moravec's utopia of 'mind children' projects a transplantation of our mental capability into machines, so that intelligence and information become independent of biological embodiment, and that the body, just as the brain, may degenerate into the dross of an immortalised mind. ${ }^{2}$ Then JeanFrançois Lyotard's Parisian exhibition 'Les Immatériaux' (1985) staged this tendency towards immaterialisation as a topos in the borderland of science and art, significant for out time. ${ }^{3}$ The assumption that the informatisation of the lived-in world leads to a dematerialisation is accompanied by a rhetoric of disappearance: the unity-endowing narrations drop away, the senses dwindle, the signs lose their referents, reality evaporates into hyperreality. Our culture's unconcealed interest in soma - be it in terms of athletical stylisation of the body or as an endorphin-raising, extreme bodily experience - can then be interpreted as a compensation and counter movement to this apparent disembodiment which is supposedly brought about by new media: a fascination both confirmed and approved of simultaneously insofar as these bodily practices are only possible in spaces that are three-dimensional and hazardous, thus real and not virtual.

The following considerations should be taken as a critical comment of the presumption that new media brings about a dematerialisation and disembodiment of our civilisation. The idea of the disappearance of the body under conditions of virtualisation falls short. It is not the dissolution of the body taking place under these conditions, but rather its splitting into both a human body and a data body, its doubling into a physical and a semiotic body. Occurrences in virtual reality - we hereafter make use of the expression

McLuhan 1968, p. 43

Moravec 1990

Lyotard 1985

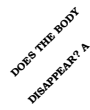


'virtual reality' as a technical term ${ }^{4}$ - are not simply due to a disembodiment, but to the sublime transformation of the body into flesh body and sign body. How can we trace this transformation? Spatiality and the principle of locality play a role. Descartes defined matter by spatial extension and therewith specified as the concept of body that which occupies a well-defined place in space. If now the conception of the body is subject to change, then this change must - in some way - have to do with a transformation of space. The metamorphosis of 'corporeality' under the condition of virtualisation relates to a metamorphosis of 'spatiality' itself.

\section{Vertigo in virtual space?}

There is a remarkable phenomenon which Jay David Bolter calls attention to: ${ }^{5}$ there is barely one sensation which more sustainably and radically asserts our having a body and its actual determination by its position in space than the phenomenon of vertigo. People who suffer from acrophobia are hardly capable of stepping on high balconies, climbing towers, or taking transparent lifts. Being exposed to such circumstances causes somatic afflictions which can assume the characteristics of pathological anxiety reactions: heart complaint, sweating hands, feeling of oppression, paralysis. ${ }^{6}$ Yet fear of heights is only perceived when one's body is actually placed in an elevated position. If virtual realities depend upon disembodiment, acrophobics who step on a virtual suspension bridge or enter virtual lifts via immersion techniques should not suffer from any physical discomfort. However, that is not the case. At Georgia Institute of Technology, three computer-generated virtual spaces were designed: a balcony situated high-up and providing a downward view, an open lift, and building-to-building suspension bridges. Acrophobia patients who entered these virtual installations did not only exhibit each somatic symptom but were even cured with the aid of these virtual spaces. Thus, the mere simulated presence of the body in the data world is perceived as a real presence and triggers physical, anxious reactions in one's own body.

How can that be? Bolter, with good reason, suspects this is not due to realistic depiction, for the phobia producing virtual realities are strongly simplified and at best coarsely operating with photo realistic, illusionary techniques. Rather, what counts is the fact that the setting 'stepped into' reacts to

4 By the term "virtual reality" we refer to technologies which allow for the integration of a user into computer generated environments, therewith enabling him to interact with the data universe. On this term, see Bühl 1996, p. 53.

5 Bolter 1996

$6 \quad$ Hodges et al. 1995

\section{8}


the user's respective body movements. Obviously, an interaction takes place between the user's physical body, fitted with a data suit and a head-mounted display (HMD), and his virtual body, situated on the suspension bridge. But this virtual body is only present in terms of the respective viewpoint from which the virtual environment presents itself to the user's eyes via HMD. Hence, in this case, the interaction is only present and embodied in terms of the perspectives which are constantly changed in respect to the movements of the user's physical body. Nevertheless, whilst the actual body only seems to be high up, the semiotic body - taken to be a point of view from a certain altitude - is actually present on the suspension bridge.

Let's have a closer look at this relation between virtuality, illusory placing and interaction.

\section{What does 'virtuality' mean?}

Elena Esposito originated the idea of associating 'virtuality' with illusory placing thereby introducing the well acquainted phenomenon of mirror images to explain 'virtual'. 'Virtual' is a term in optics which refers to images not based on light rays spreading linearly. Reflections, for instance, are virtual, insofar as they convey the impression of the mirrored objects as if they were actually located behind the mirror surface. Thus virtuality not only produces illusory objects, but provides real objects with illusory placings. In this way, seeing things from front and behind simultaneously, or seeing oneself with the eyes of the others, becomes possible. Even so we have no chance of stepping into the mirror image. We have to change the world in front of the mirror for the world in the mirror to be changed. Indeed, reflections are images, but not signs of the reflected objects. ${ }^{8}$ By extrapolating the mirror metaphor we can make a first attempt to grasp what 'computer generated virtualisation' means. Imagine that there is a technology (a) allowing us to step into the mirror world and to interact with the mirrored objects, whilst (b) what is mirrored is not simply things, but signs resp. symbol worlds. Computer generated virtual realities are immersive reflections of symbolic universes, in which a user can interact with symbol structures.

However, there is a bottleneck that must be passed through from the real to the virtual and vice versa, and this is only achievable by semiotisation. For the user this means that an immersion in the virtual world can only succeed provided a mapping of his corporeal body to a data body, which acts as an arbitrary symbolic (re-)construction of the viewpoint and movement of the physical body, is carried out. This can be accomplished by information

$7 \quad$ Esposito 1995

$8 \quad$ Eco 1988 
technologically upgrading the body with a data suit, helmet and gloves, or more subtly, via scanning or detecting the body by means of video cameras. What it comes down to is that positions and movements of body parts are recorded, digitised and made present by a "sign body" in virtual space as a representation of the physical body. This presence of the "sign body" can be implicit in terms of the viewpoint which determines the respective perspective under which a scenery appears as a function of the user's ocular movements. Or this presence can be explicit in terms of an arrow, a stylised hand, or any fictional figure which becomes part of the virtual scene. In any case the user's body exists as twofold, the physical body and the semiotic body. This duplication only succeeds since "flesh body" and "sign body" are connected by a bilaterally permeable, electronic umbilical cord.

But what gets interchanged by means of this "umbilical cord"? All virtual reality input technologies come down to the transmission of ocular, manual, facial and bodily movements. Usually, by "body" we mean something which has a specific position in space and time. Regarding bodies which change position we speak of movement. Movement, like the body itself, is determined by the principle of locality, insofar as movement invariably relates to a change in position of a body. Therefore, the user's presence in three-dimensional space and his performing actual movements - however limited the latter may be - is the condition for his semiotic body to become active in cyberspace at all. Thanks to the computer's computational power, the bodily movements are transferred to the data body so fast as to be unnoticeable to human perception, any sense of a transmission vanishes. Thus the user's semiotic body experientially does not figure as a counterpart to his physical body. The intimate spatiality, in which bodies relate to one another positionally by "here" and "there", does not hold anymore for the relation between physical and semiotic body.

It is exactly this exemption of the principle of locality concerning the data body which is the artistic message of the interactive computer installation The Trace, Remote Insinuated Presence, presented in 1995 at the Contemporary Art Fair in Madrid. ${ }^{9}$ Within this work, which is described by the artists as "tele-embodiment" ${ }^{10}$, participants who are located at separate places can, at their respective place, encounter the other's semiotic double. The physical body of one participant and the semiotic body of a second can approximate or evade one another, and eventually occupy the same place by the coincidental positions of the real person in three-dimensional space and the virtual person in telematic space. That way, real body and data body can "merge" resp.

9 This installation was presented by media artists Rafael Lozano-Hemmer and Will Bauer at Contemporary Art Fair in Madrid: Lozano-Hemmer 1996.

10 "Tele-embodiment is the technically supported act of being in spatial and temporal coincidence with other humans." Lozano-Hemmer 1996, p. 142. 
incorporate: they coincide. The principle (valid for physical reality) that two bodies cannot be in the same place at the same time does not apply anymore: The "flesh body" of one person, and the "sign body" of another are in the same "position". The imperative of distance does not hold. 'Illusory placing', which we introduced as a defining criterion of the virtual, consists of just this trick of zero distance.

But is that which is placeable in an illusory manner still a body at all? And is where it is placed, still a space at all? Differently put, if we continue calling the data body "body" and calling virtual spaces "spaces", how does the concept of body and the concept of space have to change for such designations not to become meaningless? Answering these questions remains a research task, but at least some directions emerge. For one thing, the conceptual transformations have to do with the altered relation between space and time - space here taken as three-dimensional, physical space - and for another thing, they have to do with the relation between space and person space here taken as social space.

\section{Space and time: The implementation of time into sign configurations}

What does the proficiency of computer generated virtuality consist of? The buzzword "interactivity" can show us a way. What "interaction" means is familiar from verbal conversation: 'Ego' and 'Alter' reciprocally refer to one another in what they say and how they say it. And we are just as acquainted with the intervention of writing to split up communication and interaction: wherever is written, the readers are absent, wherever is read, the author is. Indeed the symbolic register of literality (we can also say, cultural techniques) rests upon our ability to produce, look at, convert, interpret and erase signs. And yet they still elude interaction. We know about interacting with persons, with animals, too, and even with things on a limited scale, but an interactivity involving symbols is unknown to us. And that does not only bear on our dealing with texts, but likewise includes our relation to images. ${ }^{11}$

Is it by chance that spatiality is the fundamental medium of this symbolic register of literacy? A complex link between the culture of literacy and the

11 However, there is a misunderstanding to pre-empt: the dispension of interaction is not just lack and loss, but a culture conveying achievement which opens up new scopes to our cognitive and aesthetic world-relatedness. In conversational interaction among physically present persons, characterised and also blurred by asymmetries of charisma and power, it is just its interruption which in the form of solitary writing and reading profoundly promoted an individuality in the sense of the formation of an idiosyncratic perspective, critical faculties towards the truth claims of texts, as well as an insight into the difference and plurality of interpretational possibilities. 
medial order of spatiality is touched upon here. There are several phenomena which testify to such a connection:

(1) For one thing, there is the privileging of eyesight, dating back to Greek Antiquity. ${ }^{12}$ This, however, is a sense by which the manifold can be surveyed side by side, i.e. simultaneously, and whose capability furthermore increases, not diminishes, with the distance to the viewed object.

(2) For another thing, there is the - mostly remaining implicit - identification of language with script (Schrift): nearly all theories of language draw their idea of language from the model of script, which, however, is a language modality which makes use of the potential for representation of spatio-visual configurations by which language advances to an image, namely to a script figure (Schriftbild).

(3) And lastly, there is the fact that we even represent time itself only by means of visual schemes, be it a point of time, an arrow of time, or a space of time: whenever we indicate temporality, time is spatialised.

The use of computers presents a kind of cultural technique inasmuch as the cultural techniques of literacy such as reading, writing and arithmetic rest upon the exclusion of interaction with symbols, whereas that of computer utilisation, however, rests upon the inclusion of interacting with symbols. This ability to interact with symbol structures - and that is the supposition that it comes down to - becomes possible only by the implementation of time into symbolic configurations. Well, how is that to be understood?

Virtually all metaphors used to describe the forms and practices that have arisen in dealing with information called on conceptions of space (be it the "data universe", the "net", or the "desktop") as an organisational principle of a computer's user interface. Yet the assumption of this preference for the semantic field of spatiality does not account for the future impulses by which computers will shape our access to and handling of information - impulses which consist in the implementation of temporality into forms of symbols we traditionally organised as spatial relations. Writing, text and the book: of these media it is a characteristic - unlike the fluid word, barely uttered, already fading away - to display a time-resistant stability. Although each reading process creates a unique text for the reader and, during repeated reading a varied one as well, the texture conceived as a legible structure of significants remains stable. Therefore, if the transition ${ }^{13}$ from orality to literacy in medial

\section{Riedel 1984}

13 The word "transition" here does not mean a substitutional relation of orality by literacy, of which there is none in this naive form, but is meant in the sense of a systematic distinguishability. 
respects can be described as a spatialisation, then the transition from literacy to telematics lies in a temporalisation.

David Gelernter assumes that we are standing at the verge of a period in which computers mainly "deal with palpable time - with visualised, concrete time." ${ }^{14}$ There is something static and immanent to space, yet time flows (by). The fluent current, i.e. no longer the cabinet and the document kept therein, becomes the essential organisational principle of information. If temporality becomes inherent in data structures - this being Gelernter's speculation - information which is accessible and processable via computers does not remain in the shape of "documents" but takes on that of "cyber bodies". It is then organised like a "life stream". Approaches to a non-Cartesian conception of the body become visible here: a body is taken as an entity in flow which is not defined anymore by its position in space, but by its changing in time.

The basic idea of this speculation, that the implementation of time into data structures would make a significant difference between the "register of textuality" and the "register of digitality", already becomes unspeculatively evident with, for example, image-generating techniques in computer generated numerical simulations, which fulfill visualisational functions in the sciences. Gabriele Gramelsberger showed ${ }^{15}$ the basis of this kind of simulation to be a novel form and function of script, which she designated "digitised script". By means of digitised script it becomes possible to not just describe, as with phonetic script, nor to just calculate, as with formal, operational script, but to dynamise system flows in order to render temporal processes functionally representable, and computer animated images analogously presentable as well. This dynamisation of data structures in numerical simulation rests upon the fact that "points in time become real numbers which are defined by infinitesimal processes of approximation": ${ }^{16}$ thus, time becomes integrated in mathematical - and with this also symbolical - structures.

This simulation technique also allows for user movements - whether that involves eye movement, facial expression, gesticulation, or whole body movement - to be captured in a way that movement of the physical body becomes simulateable in virtual space by the data body. This data body is "corporeal" not only because it bears a relation of mapping to the user's body, but also because time is implemented into the symbolic structures of this representation as well as into the symbolic environment itself. The symbols have become dynamical, they are incarnations of time, and it is just that which enables novel interactivity with them.

14 Gelernter 2000, p. 59 (translated quoting)

15 Gramelsberger 2002, p. 75

16 Gramelsberger 2002, p. 71 (translated quoting) 


\section{Space and body: the constitution of corporeity by central perspective and virtual reality}

Through his distinction of "res extensa" and "res cogitans", Descartes did not only determine the "body" by its difference to mind, but at the same time he grasped the notion of matter as spatial extension, i.e. identifying the physical body with a geometric body.

Now if the data body in the web owes its corporeity to the implementation of temporality and motion, does that make virtual reality a phenomenon which undermines the Cartesian body conception? One might think so. Yet the situation is more complicated with regard to current techniques of virtual reality, since the virtual body is dynamisable only insofar as the real body, placed in three-dimensional space, gets reduced to a "Cartesian body par excellence". How is that to be understood?

At this point, a revealing analogy between virtual reality and the technique of central perspective lends a helping hand. With his conception of matter, Descartes philosophically realises that which central perspective effectuated with regard to the early modern body image. Usually, depiction employing central perspective appears as a "natural" representation resting upon an imitation of the laws of the visual process by the rules of artistic creation. But space in central perspective is an infinite, continuous, homogenous, and thus mathematical space which to no degree coincides with the psychophysical space of human corporeity, to which top and bottom, left and right, front and back are precisely not homogenous. Linear perspective creates a visual syntax, in the medium of which the body becomes determined and visualised in a new way: bodies - as well as their spacings, by the way - rank as incarnations of geometric proportions by which they are homogenised and rendered comparable. The body is embodied mathematics. Accordingly, the representation of the body in a picture exercising central perspective is not founded anymore on the significance and relevance of the depicted persons, but exclusively depends on their position in space put into an arithmetical relation to the immobile eye of an external observer.

Maybe the 'family likeness' between central perspective and virtual reality can now become clear. Regardless of the crucial difference that tableaux rest upon separation whereas virtual realities rely on the concurrence of representation and interaction, there is a similarity. It consists in the fact that what is considered a body does not only attain representation, but at the same time is constituted by techniques of representation. The doubling into the physical and the semiotic body as a condition for interacting with virtual realities means that the body of the user has to be transformed into a purely physical body. The coordinates of the moving physical body are transferred to its semiotic double in electronic space. The user "counts" as a mere body-in-motion. 
Like central perspective transforming the resting body into something fundamentally characterised by its spatial position, virtual reality transforms the mobile body into something ascribed by its digitisable motion grid. So, would it not be true to say that the clue to virtualisation also lies in disembodiment and depersonalisation, and not solely in the dissociation of physical body and data body?

\section{On the transformation of social space in telematic communication}

\subsection{Script as a medium of synchronicity: the "pseudonym-isation" of communication}

Now we have to focus our interest on another phenomenon concerning the use of computers. This time it is neither about virtual realities, in the sense of immersive data spaces, nor their relation to the user's three-dimensional, physical space. What it is about is the telematic, synchronous communication between spatially separated persons, taken as the genesis of a social space of virtual communities.

Social spaces come into existence through communication; what they are made of are all conditions whose 'being' rests upon 'being acknowledged'. From the perspective of acknowledgement relations, humans in social spaces do not just rate as a biological species, nor just as corporeal beings, but rather as persons. If computer mediated telecommunication leads into a virtualisation of social spaces, what does that mean, then, for the status of the communicators' personhood?

In order to find an answer to that, we have to look at which shape the semiotisation takes, i.e. the inevitable passing through the bottleneck when entering into online communication.

First, script becomes the medium of synchronicity. ${ }^{17}$ Usually, script disrupts communication: writing and reading disengage not only from the immediateness, spontaneity, irreversible fluidity of actual communication, but also from the communicators' entanglement. Between the production and reception of texts a time interval intervenes. And that even holds for email or partaking in Usenet ${ }^{18}$, both of which are kinds of asynchronous communication where the participants are not simultaneously involved in communication. This changes with telematic communication in which script - or more precisely the use of keyboard and display - obtains a new purpose, insofar as it

17 As to the altered role of writing, see Bolter 1997; Sandbothe 1997.

18 Usenet taken as an information exchange, where topic-specific articles are interchanged in newsgroups. 
enables the simultaneous exchange between spatially absent persons. With this, script enters into functions which, in face-to-face conversation, voicebound speech does fulfil. Yet unlike the indexically meaningful voice, which in speaking always asserts the trace of the body, the script figure (Schriftbild) entered via the keyboard draws on the potential of anonymisation that is linked to script as an option. Due to the relatedness of online communication to script, those partaking in it appear pseudonymously.

We can distinguish two facets of this "pseudonym-isation": (1) an arbitrarisation of the name, and (2) a staging of personal identity. As to (1): no nameno account! Having an addressable name is required for participating in web interaction. In ordinary life, our proper name is an attribute of our personality. It renders ourselves identifiable and gives us a singularity - before any biological, psychic or social development of individuality. The proper name assigns us a well-defined place in social space and social time. We do not create our proper name, but we receive it. And it endows personal identity just by virtue of that dimension in which it is not left to our power of disposition. This is quite different for the name under which we act in the web: it is chosen by ourselves, a product of a self-determined staging. This name provides an attributability which can permit remaining anonymous to the person connected to it. This name is a depersonalising one. As to (2): the choice of a name is complemented by an artificial identity. By the @describe me as command we project and produce an identity staged according to outer and inner attributes. The arbitrary character of these "self"-descriptions comes glaringly to light with the @gender command. Admittedly the web identity calls for a specification of gender all along, but which "gender-flag" is set is left to the participants.

\subsection{The division of person and persona}

So we can see from the start, the mode of writing in online communication facilitates the operation of what are no longer persons but rather arbitrary descriptions of persons, i.e. staged identities behind which all the same programmes can be concealed. We shall label this personification of arbitrary descriptions, 'persona'. 'Persona' is derivative of 'per-sonare', "to sound through" and originally means the mask through which the ancient actor speaks his role. So, does the splitting into a real person and a virtual persona imply a theatricalisation of computer generated communication? Does telematic communication become a procedure related to proceedings on a stage? 
Let us start from one popular case that has been frequently discussed in the debate on the usage of computers. ${ }^{19}$ Under the name of 'legba' a woman from Seattle acts in a MUD ${ }^{20}$ on the Internet. A participant going by the alias of 'Mr. Bungle' succeeds in cutting the real woman's control over her alias existence by use of a programming trick to then involve her in a violent pornographic interaction. For the public, reading along interestedly, the impression is conveyed that 'legba' does not only consent to her simulated rape but actively partakes in it. After another persona acting under the alias of 'starsinger' is driven to simulated self-mutilation by 'Mr. Bungle' using the same hack, a 'wizard' who has insight into the 'lower' code interrupts the affair and throws 'Mr. Bungle' out of the virtual scene. Then, via a mailing list the woman from Seattle makes the events known to the web public; further, the virtual community builds an ad-hoc government, which ostracises 'Mr. Bungle', whose personal identity of course remains unknown.

So what is the moral of the story? All acting in the web is acting by signs and thereby draws on the difference between 'word' and 'thing'. So the obnoxiousness of this episode does not lie in the description of rape - for the description of rape is not rape, at best a piece of pornography originated with this. The obnoxiousness lies in the violently interrupted influence of the real person over her virtual persona.

Yet if that is the case, a crucial difference shows up between a virtual communication environment and theatrical events on the stage. An actor is indeed responsible for good or bad acting, however, not for whether he plays the part of a good or bad character. But even so, a real participant in online communication stays in connection with his artificial identity in such a way that his responsibility for what he does in communicating, even in the web, is not suspended in principle. Nevertheless, the constitutional "pseudonymity" creates a particular state of affairs that results in the development of normative practices in virtual communities which exclusively refer to the web personae and by no means - see the treatment of 'Mr. Bungle' - to the real persons they are associated with.

\subsection{On universal pragmatic theory of communication}

Let us take a step towards contemporary speech act and communication theory. Universal pragmatic theory of communication as elaborated by Karl Otto Apel and Jürgen Habermas typically links John Langshaw Austin's idea to grasp speaking as a kind of doing, with the idea that a speaker can count as a personification of claims to be argued formal-rationally, i.e. a speaking

19 Dibbel 1994; Turkle 1995, pp. 250-254; Sandbothe 1998

20 It is the LamdaMOO created by Pavel Curtis (Xerox PARC). 
situation being of sublimely juridical resp. contractual structure. Basically, this subliminal juridification of communication in universal pragmatic communication theory is about explaining the detachment of the factual and the relational aspect in speech by the fact that in speaking we raise validity claims of truth, truthfulness and correctness, which the addressed can then - with good reason - accept or reject. The power of the better argument is the sole criterion for acceptance or rejection. And it is language itself which - by distinguishing 'communication' from 'discourse', by switching between these two levels, and by enabling an interruption of communication and entering into discourse - opens the possibility of rationally negotiating and deciding the validity claim at issue whenever disagreement arises in the spontaneous flow of communication. Discourse becomes the place where - after Habermas - abstaining from all corporeal, psychological or social differences, we are considered the mere personification of the uniformly distributed ability to defend or overthrow validity claims by arguing. ${ }^{21}$ Speaking under the idealised conditions of discourse becomes the possibility to be right. This is what is meant by the latently juridical structure of communication theory.

\subsection{Personae as participants in virtual communities}

Let us now come back to the question of the "de-personalisation" of web communication, by reinterpreting the separation of person and persona from the viewpoint of communication theory.

If the performative dimension of speech is grounded in an inter-subjective structure of approval by virtue of which participants in communication are personifications of rationally negotiable validity claims, then in anonymised web communication this performative dimension is effectively dispensed: in telematic communication, it is only spoken and not acted. This is not surprising, considering that the semiotisation constitutes the condition of the possibility of web presence resp. data existence. From this viewpoint, telematic interaction between personae indeed belongs to the type of communication which the originators of speech act theory excluded from their reflection as a form of 'parasitic communication', since here - as in speaking on stage - the very propositional-performative double structure of communication is suspended.

But at the same time, speech act and communication theory make use of idealisation assumptions which seem to be fulfilled exactly in virtual, not in actual life-world communication. Included in this, for example, is that in speaking we have to act as beings that are bared of all corporeal, gender-, social and geographic differences and who merely have to behave as par-

21 Habermas 1984, p. 353

\section{8}


ticipants provided with equal chances in communication. Where, if not in a disembodied, "pseudonym-ised", computer mediated interaction, is such a condition given? ${ }^{22}$

Regarding the form of communication emerging under such conditions, two things attract attention: (a) whereas in the theory of universal pragmatism, "to communicate" and "to argue" concur - at least in discourse - this is not the case in web communication. Communicating here is not arguing, but is a kind of reference to other personae, by responding to others and therefore becoming a participant. The maxim of web communication lies not in argumentative rationality, rather in interactive connectability. (b) Accepting 'the other as participant in interactive events' constitutes a kind of acknowledgment relation that entails the personae to actually form respective issueoriented virtual communities, which then develop their own kinds of rules. If, for instance, in order to attract attention a novice within a certain MUD is overly hasty with a 'whuggle' i.e. a virtual hug, this violates the web-etiquette and will lead to the novice being overlooked rather than integrated into the chat.

When relatability and 'being present' become the point of reference for communication, it has consequences for the modality of community-fostering possibilities of regulation and sanctioning. If - as Mike Sandbothe rightly emphasises $^{23}$ - it is correct that the internet does not constitute an anarchic space, but rather that proper methods of sanctioning misconduct have evolved, then the specificity of these methods consists just in influencing this very relatability and 'being present' and, if need be, in prohibiting it. The scale goes from being ignored by the participants to actual expulsion by a 'wizard'. At all times, however, it is a question of a persona being accepted as a playmate or not. Communicative interaction in virtual communities has the status of game moves. ${ }^{24}$

The question arises whether this phenomenon of our communication (aiming as it does, less at the defence of validity claims than at the avoidance of discontinuation of interaction), does not apply to any communication. Moreover, does the divergence of person and persona indicate that in our 'ordinary lives' too, our personality emerges from the sublime interplay of the person individuated by its proper name and the persona staged by us?

22 Unquestionably, other criteria of differentiation in terms of technical expertise arise.

23 Sandbothe 1998, p. 314

24 For an interpretation of virtuality in context of the conception of "game" see Adamowsky 2000. 


\section{Conclusion}

The starting point of our reflections was the question whether virtualisation leads to dematerialisation, disembodiment, and de-personalisation, a point about which we were decidedly sceptical. Our argumentation comprised of two steps: (1) virtualisation as a possibility of interactivity with symbol structures. The practices of literacy familiar to us rest upon our ability to create, transform, store, erase, but above all, interpret signs. Yet direct interaction with symbolic worlds is excluded by these same practices. Facilitating interactivity with semiotic structures is nevertheless possible through computermediated virtualisation. And (2) semiotisation of the user as a precondition of acting in cyberspace. The user, however, can enter into a synchronous reciprocity with signs if and only if he himself is subject to a semiotic metamorphosis. What this semiotic transformation amounts to has to be answered differently for the case of virtual reality on the one hand, and that of telematic communication on the other. (a) To plunge into virtual realities, the user has to split up into a physical body that 'counts' as an embodiment of a computable motion grid, and a data body which, in virtual reality, acts as a symbol structure. The movement of the physical body becomes the condition of the possibility of activating the data body. 'Flesh body' and 'sign body' are distinguishable but correlate and, by that interplay, introduce the question for the modification of our conception of the body. (b) To partake in telematic communication, the user has to assume a self-staged identity and split up into a real person and a virtual persona. Insofar as the personae depend on acknowledgment as participants in online communication, virtual communities develop their own standards and practices of sanctioning, which however assume the character of game moves, as the exclusion of a persona from the communicational events constitutes the most radical kind of sanctioning.

An edited re-working from Raum - Wissen - Macht, eds. Rudolf Maresch and Niels Werber, Frankfurt: Suhrkamp 2002, pp. 49-69 


\section{References}

Adamowsky, Natascha (2000): Spielfiguren in virtuellen Welten, Frankfurt a.M.: Campus.

Böhme, Hartmut (1996): „Vom Cultus zur Kultur(wissenschaft). Zur historischen Semantik des Kulturbegriffs«. In: Renate Glaser/Matthias Luserke (Eds.), Literaturwissenschaft - Kulturwissenschaft. Positionen, Themen, Perspektiven, Opladen: Westdeutscher Verlag, 48-68.

Bolter, Jay David (1996): „Virtuelle Realität und die Epistemologie des Körpers«. Kunstforum international 132, 85-89.

Bolter, Jay David (1997): "Das Internet in der Geschichte der Technologien des Schreibens«. In: Stefan Münker / Alexander Roesler (Eds.), Mythos Internet, Frankfurt a.M.: Suhrkamp, 37-55.

Bühl, Achim (1996): Cybersociety. Mythos und Realität der Informationsgesellschaft, Köln: PapyRossa Verlag.

Dibbell, Julian (1994): »A Rape in Cyberspace; or, How an Evil Clown, a Haitian Trickster Spirit, Two Wizards, and a Cast of Dozens turned a Database into a Society«. In: Mark Dery (Ed.), Flame Wars. The Discourse of Cyberculture, London: Duke University Press, 237-262.

Eco, Umberto (1988): Über Spiegel und andere Phänomene, München/Wien: Carl Hanser.

Esposito, Elena (1995): »Illusion und Virtualität: Kommunikative Veränderungen der Fiktion«. In: Werner Rammert (Ed.), Soziologie und künstliche Intelligenz. Produkte und Probleme einer Hochtechnologie, Frankfurt a.M./New York: Campus, 187-216.

Gramelsberger, Gabriele (2002): Semiotik und Simulation. Fortführung der Schrift ins Dynamische. Dissertation FU Berlin.

Habermas, Jürgen (1984): Was heißt Universalpragmatik? In: Jürgen Habermas, Vorstudien und Ergänzungen zur Theorie des kommunikativen Handelns, Frankfurt a.M.: Suhrkamp, S. 353-440.

Hodges, Larry F. / Rothbaum, Barbara O. / Kooper, Rob/Opdyke, Dan/Meyer, Thomas / Graaff, Johannes J. de/Williford, James S./North, Max M. (1995): »Presence as the Defining Factor in a VR Application: Virtual Reality Graded Exposure in the Treatment of Acrophobia«. American Journal of Psychiatry 152(4), 626-628.

Lozano-Hemmer, Rafael (1996): Televerkörperung in Trace. Kunstforum International 132, 141-144.

McLuhan, Marshal (1968): Die magischen Kanäle, Düsseldorf/Wien: Econ.

Moravec, Hans (1990): Mind Children. Der Wettlauf zwischen menschlicher und künstlicher Intelligenz, Hamburg: Hoffmann und Campe.

Münker, Stefan/Roesler, Alexander (Eds.) (1997): Mythos Internet, Frankfurt a.M.: Suhrkamp.

Rheingold, Howard (1995): Virtuelle Welten. Reisen im Cyberspace, Reinbek: Rowohlt. 
Riedel, Manfred (1984): »Logik und Akroamatik: Vom zweifachen Anfang der Philosophie«. Philosophisches Jahrbuch 91, 225-237.

Sandbothe, Mike (1997): »Interaktivität - Hyptertextualität - Transversalität. Eine medienphilosophische Analyse des Internet«. In: Stefan Münker/Alexander Roesler (Eds.), Mythos Internet, Frankfurt a.M.: Suhrkamp, 56-82.

Sandbothe, Mike (1998): »Der Pfad der Interpretation. Zur Medienethik des Internet«. Computer und Recht 5, 311-316.

Scherer, Georg (1998): »Person«. In: Joachim Ritter/Karlfried Gründer/Gottfried Gabriel et al. (Eds.), Historisches Wörterbuch der Philosophie, Bd. 7, Darmstadt: Wissenschaftliche Buchgesellschaft, 300-319.

Turkle, Sherry (1995): Life on the Screen. Identity in the Age of the Internet, New York: Simon \& Schuster. 



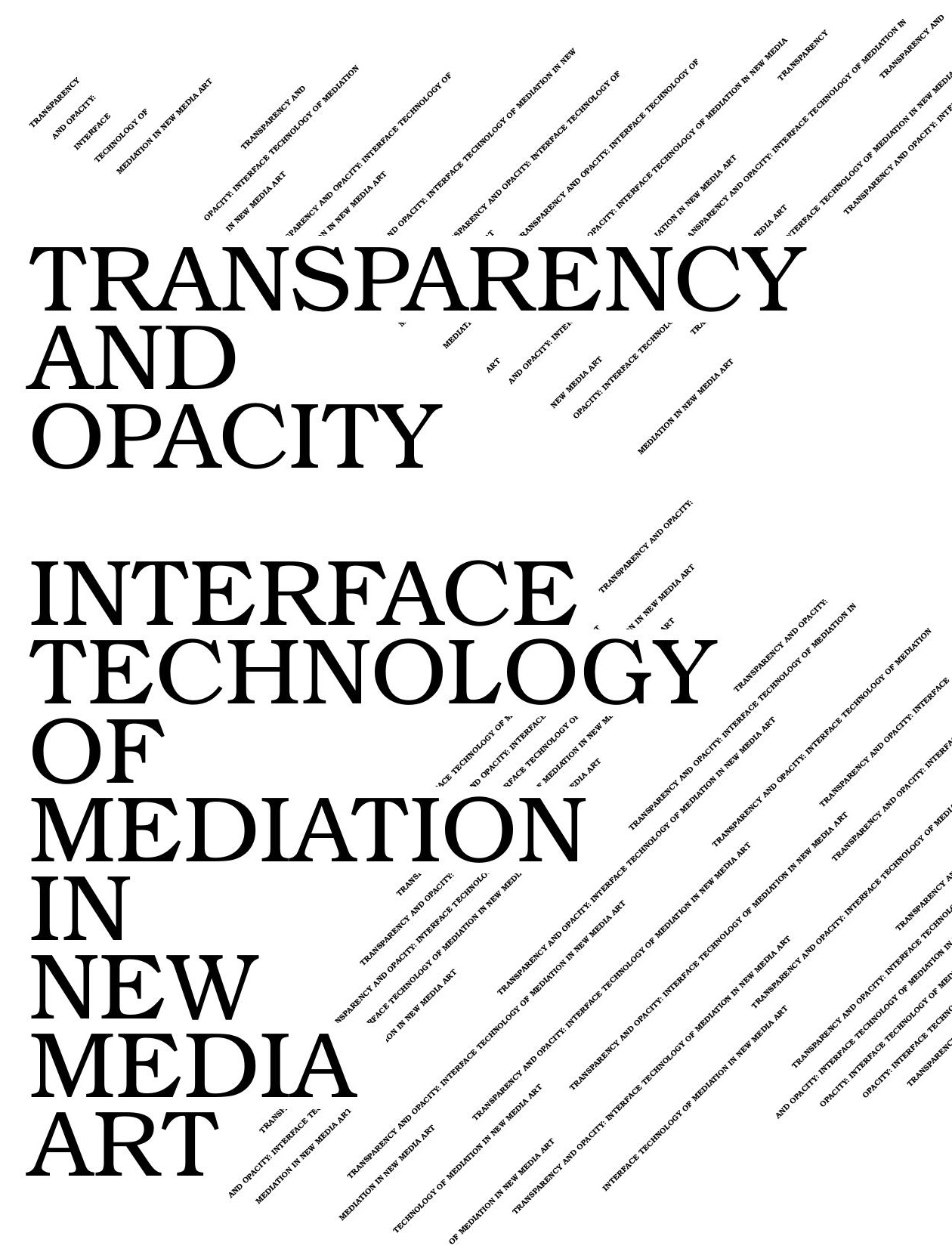

Ludwig Jäger,

Jin Hyun Kim 


\section{Mediality of artistic computer-human interfaces}

In recent decades, computer-human interfaces (CHIs) have increasingly served as a technological condition for New Media Art and at the same time as an artistic subject. The design of experimental CHIs, with help of which the observer/performer can explore a computer generated artistic environment, belongs to an essential part of New Media Art. An interface in the context of human-computer interaction (HCI) is defined as a part of the machine through which it 'communicates' with its environment. An interface mediates acts of sensory and motor processes of interacting entities. Input interfaces such as keyboards or diverse sensors make external symbolic activities accessible to a machine. The processes of machine observation in the form of 'seeing', 'listening' etc. focus on certain modalities of input data streams. A digital computer, which is capable of transforming one modality into any other, executes an action via output interfaces such as display monitors, loudspeakers, or other actuators in accordance with machine observation and interpretation of activities mediated by the input interfaces. Interface design does not only include hardware design, but also software-technological strategies of mapping from input data into output data. Mapping strategies are concerned with the question of how sensory and motor processes are related to each other by an arbitrary organisation of intermedial translation provided by digital technologies. Hence generally CHIs serve as media, not only in the sense of technical apparatuses but also in terms of performing intermedial translations which act as a condition for the emergence of meaning and/or experience. In New Media Art, CHIs can be seen in particular as media for an artistic experience. In other words, to design an artistic CHI is not only an information technological task, but also demands artistic and theoretical strategies of mediation, interfacing human and machine perception/action, which forms an artistic experience.

Starting from our thesis that an interface technology is a technology of mediation, we will investigate the mediality of artistic CHIs. In using the term "mediality" we deal with the question of how CHIs mediate 'meaning' and, in this way, shape an artistic experience while transferring signals produced by human beings and computer systems between real-worlds and computergenerated worlds. How then can a $\mathrm{CHI}$ act as a medium which is characterised in media theory as a blind spot: ${ }^{1} \mathrm{~A}$ medium fades into the background despite its material presence (e.g. the form of technical apparatus) so that the mediatised comes to the foreground, not the medium itself. Taking this into account, it is not surprising that "transparency" of a $\mathrm{CHI}$ has recently been a hot topic of interface design, even from the engineering point of view.

1 See for instance Krämer 1998, pp. 73-75.

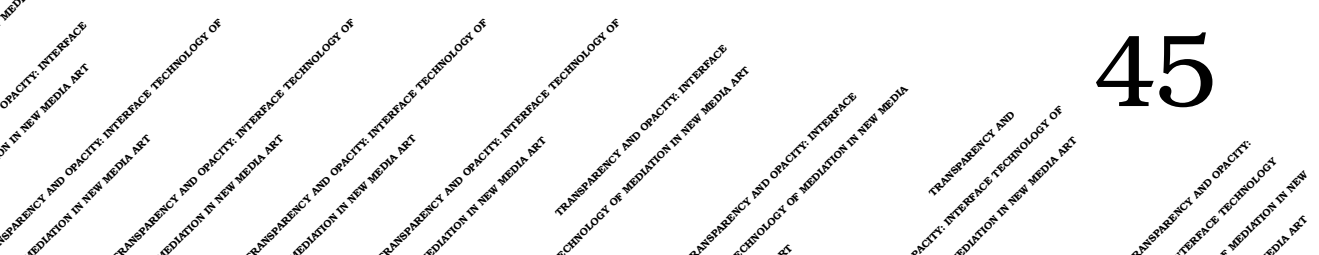


But some of the more recent arguments discussed in this context need to be reconsidered and called into question. The issue of "transparency", in the design of many of the new interfaces used for interactive music performances now take into account ideas about 'intent' which is virtually postulated as 'pre-existing' and might be ex-pressed (in terms of externalisation) by motor action in dealing with a (transparent) interface. ${ }^{2}$ In our view, dealing with 'pre-existent intent' is far from unproblematic. The core idea underlying the design of a transparent (musical) interface, which is however not related to intentionality, can be recognised in this early example posed by the composer and researcher of computer music, F. Richard Moore in 1988, on how to solve the distance between the input and output of a device mapping. He discusses this question, introducing the term "control intimacy":

Control intimacy determines the match between the variety of musically desirable sounds produced and the psychophysiological capabilities of a practiced performer. It is based on the performer's subjective impression on the feedback control lag between the moment a sound is heard, a change is made by the performer, and the time when the effect of that control change is heard. ${ }^{3}$

The recent discussions on "transparency", however, not only "provides an indication for the psychophysiological distance, $[\ldots]$, between the input and output of a device mapping", but also implies "the distance between the intent (or perceived intent, in the case of the audience) of the artist to produce some output, and fulfillment of that intent through some control action": ${ }^{4}$ the discussions focus on the design of a transparent interface, which offers a user practically no distance between intent and action. In this mode of a socalled transparent mapping, transparency is often considered an important property of an 'expressive interface', which is intended to allow a user/performer to mediate her or his (inner) artistic expressiveness. But this concept of transparency seems to deviate from the "control intimacy" developed by Moore. The latter is based on the notion that "the performer must receive both aural and tactile feedback from a musical instrument [including computeraided instruments utilising CHIs] in a consistent way - otherwise the instrumentalist has no hope of learning how to perform on it musically." ${ }^{5}$ Hence, control intimacy acts as a criterion for (musical) interfaces which are capable of responding "in consistent ways that are well matched to the psychophysiological capabilities of highly practiced performers." ${ }^{6}$ The idea of control inti-

2 Fels / Mulder 2002; Gadd/Fels 2002; Griffith et al. 2002; Marshall/Rath/Moynihan 2002; Moody/Fells / Bailey 2007

3 Moore 1988, p. 21

4 Gadd/Fels 2002

5 Moore 1988, p. 21 (supplemented by Jin Hyun Kim)

6 Moore 1988, p. 21 
macy does not take pre-existing intentions of the player into consideration, which should be conveyed by an interface designed as a transparent means. However, and crucially, the recent discourse on a transparent interface in which transparency is presumed to be one of the essential properties of an ex-pressive interface is hardly any different to a traditional concept of media which subsumes instruments, tools and devices as a transparent means of transmission of signals or representation of pre-existent entities. Should the transparency actually be seen as a property possessed per se by an interface which acts as a medium of an artistic experience? To address this issue, media theoretical discourses on transparency of a $\mathrm{CHI}$ will be surveyed.

\section{Transparency of a computer-human interface: From a New Media Theory point of view}

In different discourses on interface design and New Media Art, "transparency" has been discussed as a myth ${ }^{7}$ allowing the user to have a preferably natural experience as if it would be quasi non-mediated. In particular, the myth of transparency has been widespread with regard to virtual reality which is achieved through a three-dimensional representation providing the user a natural (three-dimensional) perspective of a computer-generated space. Immediacy is in this context a buzzword associated with transparency. Theorists on New Media, Jay David Bolter and Richard Grusin, place an emphasis on "the logic of transparent immediacy" underlying virtual reality. ${ }^{8}$ Immediacy is here related to the perceptual illusion of an immersive "experience without mediation" ${ }^{9}$ which is called forth through a "disappearing act" 10 of media. This myth of a non-mediated experience in computer-mediated communication also underlies research on virtual presence, ${ }^{11}$ the experience of being in a virtual environment, and on telepresence, the sense of being in a remote environment or virtual reality. Most research on presence is based on a realistic view of reality. Therefore the representation of a physical environment as closely as possible serves as a condition for the reality created in a technologically mediated environment. This realistic representation, (referred to by Jonathan Steuer with his term "vividity" meaning a high degree of representation or "the representational richness of a mediated environment as defined by its formal features, i.e. the way in which an environment presents

\footnotetext{
7 On this see Bolter/Gromala 2003, pp. 48-56.

8 Bolter/Grusin 1999, pp. 21-31

9 Bolter/Grusin 1999, p. 23

10 Bolter/Grusin 1999, p. 21

11 This term is adapted from Sheridan 1992 to make an explicit reference of "presence" discussed for a computer-mediated experience.
} 
information to the senses"), ${ }^{12}$ is considered necessary for transparency of a technical apparatus used for virtual reality. Transparency of a medium is constitutive for virtual presence or telepresence based on the immediacy the user attains to, which the researchers on presence, Matthew Lombard and Theresa Ditton, characterise as "the extent to which a person fails to perceive or acknowledge the existence of a medium during a technologically mediated experience." 13

Jay David Bolter, however, directs our attention towards different strategies of interface design which include not only transparency, but also its opposites such as hypermediacy. Hypermediacy offers multiple signs of representation or reflectivity reacting to the user her- or himself situated in her or his surroundings and in the context initated by compelling experience mediated by means of an interface. ${ }^{14}$ Transparency is considered a strategy which can be chosen along the continuum consisting of manifold scales of hypermediacy. Hence, transparency cannot be regarded as a property possessed per se by an interface. A property of an interface arises out of medial strategies of interface design which vary according to each artistic project and can be changed in the course of interaction.

As opposed to interface design from an engineering science point of view (which aims in general at the illusion of transparent immediacy an interface offers), media art tends to direct the user's / observer's focus towards the media designed especially for any artistic purpose along with the palette of multiple meanings of the media emerging during the user's / observer's exploration. For media art, a medium can be both a means of, and at the same time a subject for artistic projects. In New Media Art, in which interactivity comes to the fore, a CHI designed and used for an artistic purpose serves as a medium which, however, does not remain static. It is assigned a temporal dimension due to the character of works of art based on a temporally expanded interaction space. Therefore a status change of the same interface as a medium relies on interaction actively explored by the user/observer.

To make clear the idea that transparency of the media acting as a blind spot to bring the mediatised to the fore is not a property of a medium per se, in the following, some considerations on the performative logic of the medial are to be made, which are connected with the attempt to determine more precisely the procedures of media in the light of a theory of transcriptivity. ${ }^{15}$

\footnotetext{
12 Steuer 1992

13 Lombard/Ditton 1997

14 On the logic of hypermediacy see Bolter/Grusin 1999, pp. 31-44; on the strategy of interface design leading to reflexion see Bolter/Gromala 2003, pp. 62-65.

15 This theory has been developed by the first author within the scope of the German Collaborative Research Centre Media and Cultural Communication (=SFB/FK 427).
} 


\section{The performative logic of the medial: Disturbance and transparency}

The term transcriptive refers to all infra- and intramedial procedures of cultural semantics which generate meaning through the mutual co-reference of various media or symbolic means of the same system. ${ }^{16}$ In the following, the term "transcriptivity" is to be specified more precisely against the background of a media and communication theoretical model in the centre of which are the terms disturbance ${ }^{17}$ and transparency ${ }^{18}$. (At the same time, the term can certainly be understood against the background of some referential terms of New Media Theory, such as that of "cultural reconceptualisation", as used by Manovich ${ }^{19}$, or that of "remediation", as introduced by Bolter and Grusin. ${ }^{20}$ )

The model which I propose here assumes that communicative processes can be in at least two states of aggregation: (1) in that of non-disturbance, in which the medial (symbolic) means respectively used are not an issue as such, so that a direct "looking through"21 onto the semantics of the communicated is possible. A communicative state of this kind can be described as a state of medial transparency. A second state is to be distinguished from

16 Jäger 2002, in print (a) and (b).

17 Interference is not understood as a 'miscommunication', as in the communication theoretical approach of Shannon, but as the constitutive impetus of communication; on Shannon see Shannon 1949; on the history of the impact of the Shannon/Weaver "flow diagram of communication" see also Schüttpelz 2002; for a more detailed discusssion of a media theoretical concept of interference, see Jäger 2004.

18 The concept of transparency originates from the semiological discussion of the close of the 18th and the beginning of the 19th century (here see also Jäger 2004) and here is placed in a media and communication theoretical context. In the semiological tradition from which it comes, transparency does not mean that the medium becomes glass-like for the benefit of premedially existing contents, but that it virtually disappears with and in its function of constituting content. The concept of transparency therefore does not refer to the glassiness of the sign expression, but to the fact that the 'transparent' medium is dissolved in its content-constituting function, and so shifts receptive attention from the mediation to that which is being mediated. In an approximately analogous manner to our own use of the term, Bolter and Grusin speak of "transparent immediacy" (Bolter/Grusin 2001, p. 21).

19 Manovich 2001, p. 47: "In new media lingo, to "transcode" something is to translate it into another format. The computerisation of culture gradually accomplishes similar transcoding in relation to all cultural categories and concepts. That is, cultural categories and concepts are substituted, on the level of meaning and/or language, by new ones that derive from computer's ontology, epistemology, and pragmatics. New media thus acts as a forerunner of this more general process of cultural reconceptualization."

20 Bolter/Grusin 2001, p. 45: “(...) we call the representation of one medium in another remediation, and will argue that remediation is a defining characteristic of the new digital media." In the same vein, for instance p. 55: "It would seem, then, that all mediation is remediation. (...) No medium, it seems, can now function independently and establish its own separate and purified space of cultural meaning."

21 On the distinction between "looking through" and "looking at" see Bolter/Grusin 2001, p. 41. 
this, (2) the state of interruption of the transparency mode by an interactant for the purpose of fixing the attention on sign sequences communicated, and their mono- or interactive processing on a semantic stage for negotiating. A communicative state of this kind can bring about a "looking at" 22 of particular, topicalised sections of medial performance in their material presence, because these are detached from the communicative process and become the object of transcriptive attention. I would like to describe this communicative state as the state of disturbance. Disturbances mark those moments of medial communication in which the medium itself becomes the object of communicative attention.

Disturbance and transparency are to be understood as two polar functional states of medial performance which are constitutively inscribed in the process of transcription. Transcription could then be described as the transition from disturbance to transparency, from the decontextualisation to the recontexualisation of the signs/media on which the focus is placed. While disturbance as the respective point of departure actuates the transcriptive process of remediation and brings the medium as a (disturbed) operator of sense into the focus of attention, transparency can be seen as the state (in the process of medial performance) in which the respective medium disappears or becomes transparent with respect to the content which it mediatises (distributes, archives, constitutes). Disturbance and transparency therefore mark two modes of visibility which are in general mutually exclusive: the visibility of the medium and that of the mediatised. The invisibility (transparency) of the sign/medium virtually allows the undisturbed 'realism' of the mediatised, while the becoming visible of the medium, i.e. the irritation of the habitualised contexts of use, indicates the looming crisis of the ontological illusion which is then withdrawn from the mediatised objects. Realism is, as Goodman notes, relative to the media: “(...) it is determined by the system of representation standard for a given culture or person at a particular time," ${ }^{23}$ or by the medial dispositive in which the communication respectively takes place. The realism with which symbolic means carry out representation ${ }^{24}$ is higher, the more familiar (the more transparent) the means selected are. Medial displays seem realistic to us when "practice has rendered the symbols so transparent that we are not aware of any effort, of any alternatives, or of making any interpretation at all." ${ }^{25}$ In communicative states of this kind, the

22 Bolter/Grusin 2001, p. 41

23 Goodman 1976, p. 37

24 For Goodman representation does not mean "mirroring". (Pictoral) representation and (verbal) description are kinds of denotation: "Representation is thus disengaged from perverted ideas of it as an idosyncractic physical process like mirroring, and is recognized as a symbolic relationship that is relative and variable" (Goodman 1976, p. 43).

25 Goodman 1976, p. 36 
mediatisation of the real is "... obscured by our tendency to omit specifying a frame of reference when it is our own, ${ }^{26}$ or - formulated differently - when it is one of our own present (undisturbed) language games which is in use as a symbol system. Only "changes in representational practices", ${ }^{27}$ in other words effects of disturbances, only the erosion of habitual contextual frames, allow the medial relativity of the real and hence the symbolic representation system to become visible as a "way of worldmaking" 28 again. ${ }^{29}$ Crises of this kind are natural as disturbances of semantic equilibrium, not just epochal events which every once in a while distress cultural viewpoints and world views. They are first and foremost, common transitional stages which medial processes pass through according to their own logic of transcriptivity until communication in turn enters into phases of transparency.

Disturbance is then taken to mean that state in the process of a communication which has the effect that a medium (operatively) loses its transparency and is perceived in its materiality. Transparency in turn means that state in which communication is not 'disturbed', and so the medium is not in the focus of attention as a medium. Transparency can be understood for instance, in the sense in which Luhmann assumes that in the interdependent relationship of medium and form the form is visible and the medium remains invisible. ${ }^{30}$ If one were to transfer the disturbance-transparency model onto Luhmann's medium-form distinction, disturbance would be the state of a communication in which it is not the form which is observed through the (invisible) medium, but the "contingency of formations" 31 or "the free capacity of the medial substrate to make ever-new couplings" 32 that would be observed in the medium. One could also - following on from Edgar Rubin and Marshal McLuhan - say that the medial process in the state of disturbance brings the medium into the focus of attention as a figure, while it recedes into the background in the state of transparency: “(...). All cultural situations are composed of an area of attention (figure) and a very much larger area of inattention (ground). The two are in a continual state of abrasive interplay,

\section{Goodman 1976, p. 37 \\ 27 Goodman 1976, p. 39 \\ 28 Goodman 1978}

29 For a critical discussion of the media theoretical implications of Goodman's symbol theory, see Mitchell 1994, pp. 345-362.

30 On this see for example also Luhmann 1997a, pp. 190-202. For instance, the following is noted with respect to the perceptive media: "We do not see the light, but things (...). We do not hear the air, but noises." (Luhmann 1997a, p. 201; translated quoting); on this see also Krämer 1998. Following on from Luhmann, she formulates "that wherever we encounter media, we do not perceive media themselves, but only forms" (Krämer 1998, p. 76; translated quoting); also Krämer 2001, p. 157: "Moreover, the form is visible - the medium, on the other hand, remains invisible." (translated quoting)

31 Luhmann 1997b, p. 168 (translated quoting)

32 Luhmann 1997a, p. 200 (translated quoting) 
with an outline or boundary or interval between them that serves to define both simultaneously." 33 If Alfred Schütz were to be brought in, we could also describe the state of disturbance as the becoming relevant of the medium, and the state of transparency as its return to the mode of familiarity. ${ }^{34}$ What could be even more illuminative for the unravelling of the processual complementarity of disturbance and transparency than the terminological analogies provided by Luhmann, Rubin, McLuhan and Schütz is a pair of terms which has been talked about in the discourse of analytical philosophy in a context having to do rather with the logic of research, namely the terms implicit and explicit knowledge. ${ }^{35}$ In a broad sense transcriptive processes, insofar as they are understood as processes which move out of disturbance and into transparency, can then be understood as processes of "expressing”: “(...) as a matter not of transforming what is inner into what is outer but of making explicit what is implicit." ${ }^{36}$ If - as Brandom formulates - "what is expressed must be understood in terms of the possibility of expressing it," ${ }^{37}$ it is one of the constitutive conditions of implicit semantics that it - in the case of communicative disturbances - must be able to be made understandable in explicative (transcriptive) processes. Hence while in the state of medial transparency semantics is processed in the form of silent knowledge, explicative (transcriptive) actions are required when the implicit in one form or another becomes problematic or the subject of attention, and with this the medium as such comes to the fore. Transcriptions are therefore to this extent explications which under particular communicative discursive conditions are once again transferred into the status of implicit, i.e. silent knowledge. If this kind of precondition is assumed, then the medium is the mediator ${ }^{38}$ of something which - depending on the aggregate state of the communication - changes between figure and ground or between relevance and familiarity. And it is precisely this continuous changing which allows the medium to be more than an expression and a mediator of something internal: namely the explicator of something implicit which, by being explicated, changes its epistemic status to such an extent that one could say, in a sense that the implicit is not only expressed, but also constituted by its explication. The medium is then the (performative) place, the place of processing at which implicit semantics becomes explicit, only to - in the case of 'undisturbed' communication - enter

33 McLuhan/Powers 1989, p. 5; on this see also Fohrmann 2008.

34 Schütz 1971; on this see Jäger 2001.

35 On this see for example Hare 1974; Polanyi, 1966; Brandom 2000.

36 Brandom 2000, p. 8

37 Brandom 2000, p. 9

38 "Mediator" in the sense of Engell und Vogel, who understand the medium as "the middle and the median, the mediation and the mediator" (Engell/Vogel 1999, p. 9; translated quoting). 
into the state of the implicit once again, by which process the medium disappears behind the semantics which it helps to organise (without being absent). Explications are to this extent processes of (disturbance-induced) focusing and concentrating on media (and their implicit semantics) in the interest of creating (explicative) semantic effects which, when they occur, push the medium out of the focus of attention once again. They are processes of recursive self-processing, i.e. the application of communication to the effects of communication. ${ }^{39}$ I would like to call this feedback movement which operatively determines the processes of media systems recursive transcriptivity. ${ }^{40}$ It is this process of recursive transcriptivity through which the processes of disturbance and transparency are connected with each other, through which - under different medial and communicative conditions - the processes of cultural semantics are kept going, i.e. are updated through alternating stages of stabilisation and irritation in fragile states of equilibrium which can be disturbed at any time. In contrast to many media theoretical positions, the model proposed here does not assume that transparency can be seen as a constitutive property of the medial. That media tend to "become virtually imperceptible, anaesthetic" with respect to what they mediatise, ${ }^{41}$ that they remain invisible under certain conditions ${ }^{42}$, because - as Fritz Heiders formulated in 1926 - a true medium is only one through which "one can see through ... without obstruction", ${ }^{43}$ does not refer to a quasi-ontic feature of the medial, but to a particular stage which medial processes pass through in the process of recursive transcription. So the thesis which is advanced here is that the transparency of the medium is not a 'property' of the medium, but an aggregate state in which the mediatised semantics as silent knowledge is not communicatively 'disturbed'. Just as conversely, disturbance is not a parasitical defect of communication, but that aggregate, communicative state in which the sign/medium becomes visible as such, and hence semanticisible. In other words, disturbance is that state which is always connected with the need for remediation, i.e. transcription.

39 See here Luhmann's concept on the recursive "individual behaviour" of systems: "It [the term "individual behaviour"] refers to a stability reached in the recursive procedure of the application of procedures to the results of procedures." (Luhmann 1997a, p. 213; translated quoting)

40 Jäger 2004

41 Engell/Vogel 1999, p. 10; see in particular footnote 15.

42 Krämer 2001, p. 157

43 Heider 1959, p. 3 


\section{Transparency and opacity of an interface in New Media Art}

Applied to our research area of New Media Art, we can behold a performative logic of the media underlying an artistic interaction, which is based on the oscillation between disturbance and transparency of a computer-human interface. The act of recursive transcription which is constitutive for transparency of the medium emerging as an effect during a medial performance can be illustrated in cases of interaction between bodily movement and sound. In artistic interaction mediated by an interface translating bodily movement into sound, the interface operationally loses its transparency in favour of the processing of bodily-based expressions of generated sounds. In this state, the interface becomes - with its physical materiality - opaque. Every new kind of interface gives rise to partially or completely unfamiliar relationships between bodily movements and sounds. This allows a user to explore explicitly how motor and auditory feedback may be coupled. Dealing with a new interface hence includes a self-perceptive moment in the progress of artistic production. Each act within the interactive situation seems to begin with a kind of disturbance that redirects one's attention to the opacity of the interface. Opacity first comes to the foreground so that a user may generate expressiveness, appropriating the procedures of intermedial transcription, as an effect which, once achieved, shifts the interface - without making it become really absent - out of the focus of attention. In this state of transparency, the selfsignificance of an interface becomes absent. Thus, the mediating aspect of interface technology does not seem to consist in the function of an interface as a means of expressing or transferring a user's pre-existent intentions or states. Rather, the medial operation of oscillation between transparency and opacity renders an interface capable of problematising an implicit artistic intention or meaning and constituting it as an effect.

Let us have a look at some concrete strategies of the design of interfaces mediating bodily movements and sounds. One of them is oriented towards a model of physical-acoustic musical instruments. This aims to simulate or extend traditional physical-acoustic musical instruments by digital technologies. In these strategies, a bodily movement acts as a kind of trigger and controller for digital sound synthesis. The principle of digital (algorithmic) sound generation is characterised by a decoupling of the sound generation mechanism (synthesis algorithm) from its control device - this is substantially different from the mechanism of sound generation underlying a physical-acoustic, musical instrument in which sound generator - e.g. violin strings - and controller - e.g. violin bow - are closely coupled. In digital musical instruments, a mapping from the input parameter of bodily movements as a control parameter into an output parameter used for algorithmic sound synthesis, 
which is called gesture mapping (Fig. 1), therefore plays an important role for a (re-)coupling of sound control and generation mechanisms.

Such an artificially enacted relation between bodily movement and sound is constituted by transcription which may serve as a basis for mapping algorithms. Hereby, transcription relates to the inter-

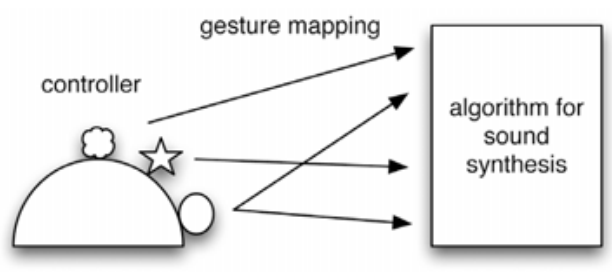

Fig. 1. Gesture mapping mediality between bodily movement and sound. When an interface is in a state of opacity, a user is required to ascertain this intermedial coherence which becomes evident in an action-perception loop taking place during an interaction.

At the outset of dealing with a musical CHI, a user/musician is able to recognise what strategies of remediation of a traditional musical instrument have been used in the design of the CHI: highlighting, refashioning or absorbing it. ${ }^{44}$ The design of the series of MIDI (Musical Instrument Digital Interface) controllers - for instance, MIDI keyboard, MIDI string and wind controller - simulating the controller of physical-acoustic, musical instruments in connection with synthetic instrument sounds, persues a strategy highlighting the musical instrument. Another group of musical interfaces called augmented musical instruments or instrument-inspired gestural controllers are designed with strategies of reshaping musical instruments.

Let us take the overtone violin developed by the composer Dan Overholt as an example of an augmented musical instrument. This controller is capable of preserving the traditions of violin bowing technique in dealing with the strings (albeit six strings instead of four). A set of embedded sensors added to this controller, however, afford extended or even new possibilities for musical control. Violin playing techniques captured for instance by optical pickups sensing the vibrations of the strings are used for the creation of expressive nuance of sounds. Other musically relevant gestures captured by diverse gesture-sensors are used to add new control dimensions to the instrument. ${ }^{45}$

As a result, musicians can play this augmented instrument with violin playing technique as is usual and at the same time explore a new playing technique. Such a musical interface might become transparent while musi-

44 The terms "highlighting", "refashioning" and "absorbing" borrow from the different strategies of remediation of older media in New Media developed by Bolter/Grusin 1999.

45 Overholt 2005 
cians are mastering the instrumental technique necessary for the combination of the usual and novel control dimensions. The "playing" of augmented musical instruments therefore requires an intensive time-consuming exercise, comparable to dealing with traditional musical instruments.

Instrument-inspired gestural controllers featuring a strategy of reshaping a musical instrument allow musicians to use a more or less traditional instrumental technique to generate and modify sound events. However, the sounds that actually emerge may likely have little to do with the instrumental gestures normally associated with their production. An example of instrument-inspired, gestural controllers is the violin-inspired controller BoSSA (Bowed-Sensor-Speaker-Array) devel-

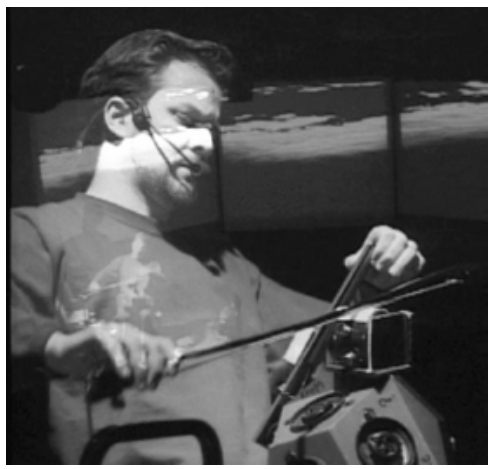

Fig. 2. Dan Trueman is playing the BoSSA. Courtesy of Dan Trueman oped by the composer Dan Trueman and Perry Cook (Fig. 2). This instrument consists of the violin's physical performance interface and its spatial filtering, audio diffusor, yet possesses neither the resonating body nor the strings. Instead it is equipped with a bow interface extended with pressure, force resistance and accelerometer sensors and virtual strings consisting of sponge divided into four parts which can be bowed. ${ }^{46}$ In this way, musicians can use a set of possibilities of bowing techniques familiar to them. But gestural data captured by different sensors while playing this interface can be mapped arbitrarily to any musical parameter so as to give rise to a change of loudness, vibrato or timbre, for instance.

As a consequence, musicians are confronted with the opacity of the controller at the outset far beyond their expectations which have been caused by the possibility of using traditional, gestural techniques. The novel relationship between instrumental gesture and sound events triggered by this gesture, however, challenges a musicians' available image underlying the playing of a musical instrument. This disturbance gives rise to a reprocessing of intermedial relationships between gestural activities used for instrument technique and auditory as well as tactile perception, in the course of which a new image of playing technique is constituted, replacing a previous one. This process of constitution of a (mental) image is due to intermedial transcription which is based on cultural semantics associated with instrumental techniques and

46 Trueman/Cook 1999 
used tone systems. Transparency of such an instrument-inspired gestural controller is achieved when the interaction renders a musician capable of becoming habituated and of feeling musical expressiveness in dealing with the interface.

Alternate controllers which do not have any affinity to a traditional musical instrument allow the user/performer to develop her or his own strategy which mediates bodily movements and sounds, since habitual behaviour adapted from an old medium cannot be applied. But some musicians tend to design such controllers

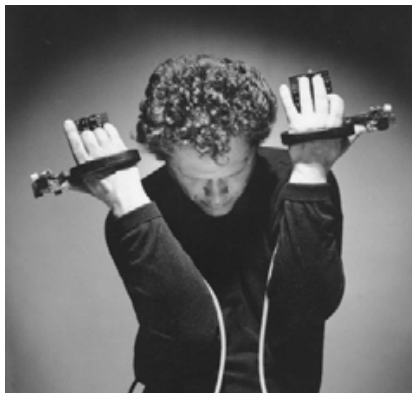

Fig. 3. Michel Waisvisz is playing the second prototype of The Hands. Courtesy of Michel Waisvisz

as instrument-like to offer a gesture mapping from musically meaningful gestures intentionally produced, into comprehensible and reproducible musical events. A well-known alternate controller The Hands (Fig. 3) developed at STEIM (Studio for Electro-Instrumental Music) since 1984 is a prominent example for instrument-like, alternate controllers. Although the shape of this interface does not have any similarity to a traditional musical instrument, it allows a musician to perform with virtuosity. A lot of alternate controllers which have been used in less musically oriented, media performances pursue a strategy of absorbing a musical instrument, so that each user - not only a musician - is required to (re)build her or his own image with regard to the intermedial relationship between bodily movement and sound. In the stage of getting to know such an interface, the medium is fully in the focus of attention. In this state of disturbance of an interface, a transcriptive process begins with problematising an implicit image and explicating it while dealing with a medial trace in the process of artistic interaction, in which the medium becomes ultimately transparent and an image explicitly explored becomes, in turn, implicit. An implicit knowledge of how to deal with each new interface is generated as an effect of embodied interaction guided by the match between bodily action on the one hand and auditory and tactile perception on the other. The user's / observer's active involvement in an unknown (artistic) world offered by New Media Art therefore acts as a condition for the genesis of an artistic experience which then comes into the focus of attention as a figure, pushing the interface into the background.

An artistic experience, which is often related to expression on the one hand and to impression on the other does not seem to be strictly separable into productive and receptive experience in New Media Art, where artists often act as observers of constituted works of art and vice versa at the same time. A person who enters into a computer-generated world acquires an artistic 
experience via a coupling of activity and passivity, production and perception, motor and sensory processes. In this way, coupled acts of sensory and motor processes have an impact on (completion of) implicit artistic expressiveness experienced by the interactant, while explicitly exploring the strategies of an interface, artificially mediating action-perception loops.

The mediality of an artistic CHI therefore consists in a reshaping and constitution of artistic experience which is initiated by a disturbed, opaque state of an interface as a medium. Each strategy of an interface mediating and modeling a relationship between bodily movement and sound proves to be based on recursive transcription which attracts one's attention towards explicating processes of artistic experience to attain to implicit aisthesis. In this way, interface technology of mediation, oscillating between transparency and opacity of the medium, accentuates the mediality of (intramental) aisthesis even by modeling intermental processes taking place in embodied and situated interaction such as artistic human-robot interaction, which require transcriptive remediation to constitute a cultural semantics of artistic experience. 


\section{References}

Bolter, Jay David/Grusin, Richard (2001): Remediation. Understanding New Media, Cambridge, MA: MIT Press.

Bolter, Jay David/Gromala, Diane (2003): Windows and Mirrors. Interaction Design, Digital Art, and the Myth of Transparency, Cambridge, MA: MIT Press.

Brandom, Robert B. (2000): Articulating Reasons. An Introduction to Inferentialism, Cambridge, MA: Harvard University Press.

Engell, Lorenz/Vogel, Josef (1999): »Foreword« [Vorwort]. In: Claus Pias et al. (Eds.), Kursbuch Medienkultur. Die maßgeblichen Theorien von Brecht bis Baudrillard, Stuttgart: Deutsche Verlags-Anstalt, 8-11.

Fels, Sidney/Gadd, Ashley/Mulder, Axel (2002): »Mapping Transparency through Metaphor: Towards More Expressive Musical Instruments«. Organised Sound 7(2), 109-126.

Fohrmann, Jürgen (2008): "'Schulinspektor”, 'binary digits' und “kulturelles Netzwerk”. Über einige Gemeinsamkeiten der Medien- und Kommunikationstheorie zwischen den 1930er und 1960er Jahren«. Modern Language Note (MLN) 123(3), 613-631.

Gadd, Ashley/Fels, Sydney (2002): »MetaMuse: Metaphors for Expressive Instruments«. In: Proceedings of the 2nd Conference on New Interfaces for Musical Expression. Online available: <http://www.nime.org/2002/proceedings/paper/gadd.pdf> (last access: October 2007).

Goodman, Nelson (1976 ): Languages of Art. An Approach to a Theory of Symbols, Indianapolis/Cambridge: Hackett Publishing Company Inc.

Goodman, Nelson (1978): Ways of Worldmaking, Indianapolis: Hackett.

Griffith, Niall et al. (2002): „Circles and Seeds: Adapting Kpelle Ideas about Music Performance for Collaborative Digital Music Performance«. In: Proceedings of the 2nd Conference on New Interfaces for Musical Expression 2002. Online available: <http:/ / www.nime.org/2002/proceedings/paper/griffith.pdf> (last access: March 2007).

Hare, Richard M. (1974): »Philosophische Entdeckungen«. In: Günter Grewendorf/Georg Meggle (Eds.), Linguistik und Philosophie, Frankfurt/M: Athenäum, 131-153.

Heider, Fritz (1959): Thing and Medium. In: Fritz Heider: On Perception, Event Structure, and Psychological Environment. Selected Papers. Psychological Issues 1(3), New York: International UP, 1-34.

Jäger, Ludwig (2001): »Zeichen/Spuren. Skizzen zum Problem der Zeichenmedialität«. In: Georg Stanitzek/Wilhelm Voßkamp (Eds.), Schnittstelle: Medien und kulturelle Kommunikation, Cologne: DuMont, 17-31.

Jäger, Ludwig (2002): »Transkriptivität. Zur medialen Logik der kulturellen Semantik«. In: Ludwig Jäger/Georg Stanitzek (Eds.), Transkribieren - Medien/Lektüre, Munich: Fink, 19-41. 
Jäger, Ludwig (2004): »Störung und Transparenz. Skizze zur performativen Logik des Medialen«. In: Sybille Krämer (Ed.), Performativität und Medialität, Munich: Fink, 35-74.

Jäger, Ludwig (in print (a)): »Transkriptive Verhältnisse. Zur Logik intra- und intermedialer Bezugnahmen in ästhetischen Diskursen«. In: Gabriele Buschmeier/Ulrich Konrad/Albrecht Riethmüller (Eds.), Transkription und Fassung. Bericht des Kolloquiums Mainz 2004, Mainz: Akademie der Wissenschaften und der Literatur.

Jäger, Ludwig (in print (b)): »Transkription. Überlegungen zu einem neuen interdisziplinären Forschungsparadigma«. In: Walter Bruno Berg/Rolf Kailuweit/Stefan Pfänder (Eds.), Migrations et transcriptions: Europe et Amérique latine de voies en voix, Freiburg.

Krämer, Sybille (1998): »Das Medium als Spur und als Apparat«. In: Sybille Krämer (Ed.), Medien - Computer - Realität. Wirklichkeitsvorstellungen und neue Medien, Frankfurt a.M.: Suhrkamp, 73-94.

Krämer, Sybille (2001): Sprache, Sprechakt, Kommunikation, Sprachtheoretische Positionen des 20. Jahrhunderts, Frankfurt a.M.: Suhrkamp.

Lombard, Matthew/Ditton, Theresa (1997): »At the Heart of It All: The Concept of Presence«. Journal of Computer-Mediated Communication 3. Online available: <http://jcmc.indiana.edu/vol3/issue2/lombard.html> (last access: November 2007).

Luhmann, Niklas (1997a): Die Gesellschaft der Gesellschaft, Frankfurt a.M.: Suhrkamp.

Luhmann, Niklas (1997b): Die Kunst der Gesellschaft, Frankfurt a.M.: Suhrkamp.

Manovich, Lev (2001): The Language of New Media, Cambridge, MA: MIT Press.

Marshall, Mark T./Rath, Matthias/Moynihan, Breege (2002): "The Virtual Bodhran -

The Vodhran«. In: Proceedings of the 2nd Conference on New Interfaces for Musical

Expression 2002. Online available: <http://www.nime.org/2002/proceedings/ paper/marshall.pdf> (last access: July 2008).

McLuhan, Marshall/Powers, Bruce R. (1989): The Global Village. Transformations in

World Life and Media in the 21st Century, New York: Oxford University Press.

Mitchell, W. J. Thoman (1994): Picture Theory. Essays on Verbal and Visual Representation, Chicago/London: The University of Chicago Press.

Moody, Niall/Fells, Nick/Bailey, Nicholas (2007): »Ashitaka: An Audiovisual Instrument«. In: Proceedings of the 7th Conference on New Interfaces for Musical Expression 2007, 148-153.

Moore, F. Richard (1988): »The Dysfunction of MIDI«. Computer Music Journal 12/1, 19-28.

Overholt, Dan (2005): »The Overtone Violin«. In: Proceedings of the 5th Conference on New Interfaces for Musical Expression, 34-37.

Polanyi, Michael (1966): The Tacit Dimension, New York: Doubleday \& Company, Inc. 
Schüttpelz, Erhard (2002): »Eine Ikonographie der Störung. Shannons Flußdiagramm der Kommunikation in ihrem kybernetischen Verlauf«. In: Ludwig Jäger/Georg Stanitzek (Eds.), Transkribieren - Medien/Lektüre, Munich: Fink, 233-280.

Schütz, Alfred (1971): Das Problem der Relevanz, Frankfurt a.M.: Suhrkamp.

Shannon, Claude (1949): »Communication in the Presence of Noise . Proceedings of the Institute of Radio Engineers 37, 10-21.

Sheridan, Thomas B. (1992): »Musings on Telepresence and Virtual Presence«. Presence: Teleoperators and Virtual Environments 1(1), 120-126.

Steuer, Jonathan (1992): »Defining Virtual Reality: Dimensions Determining Telepresence«. Journal of Communication 4(2), 73-93.

Trueman, Daniel/Cook, Perry R. (1999): „BoSSA: The Deconstructed Violin Reconstructed«. In: Proceedings of the 1999 International Computer Music Conference, San Francisco: International Computer Music Association 1999. Online available: <http://silvertone.princeton.edu/\%7Edan/BoSSA/icmc99.pdf> (last access: January 2008). 


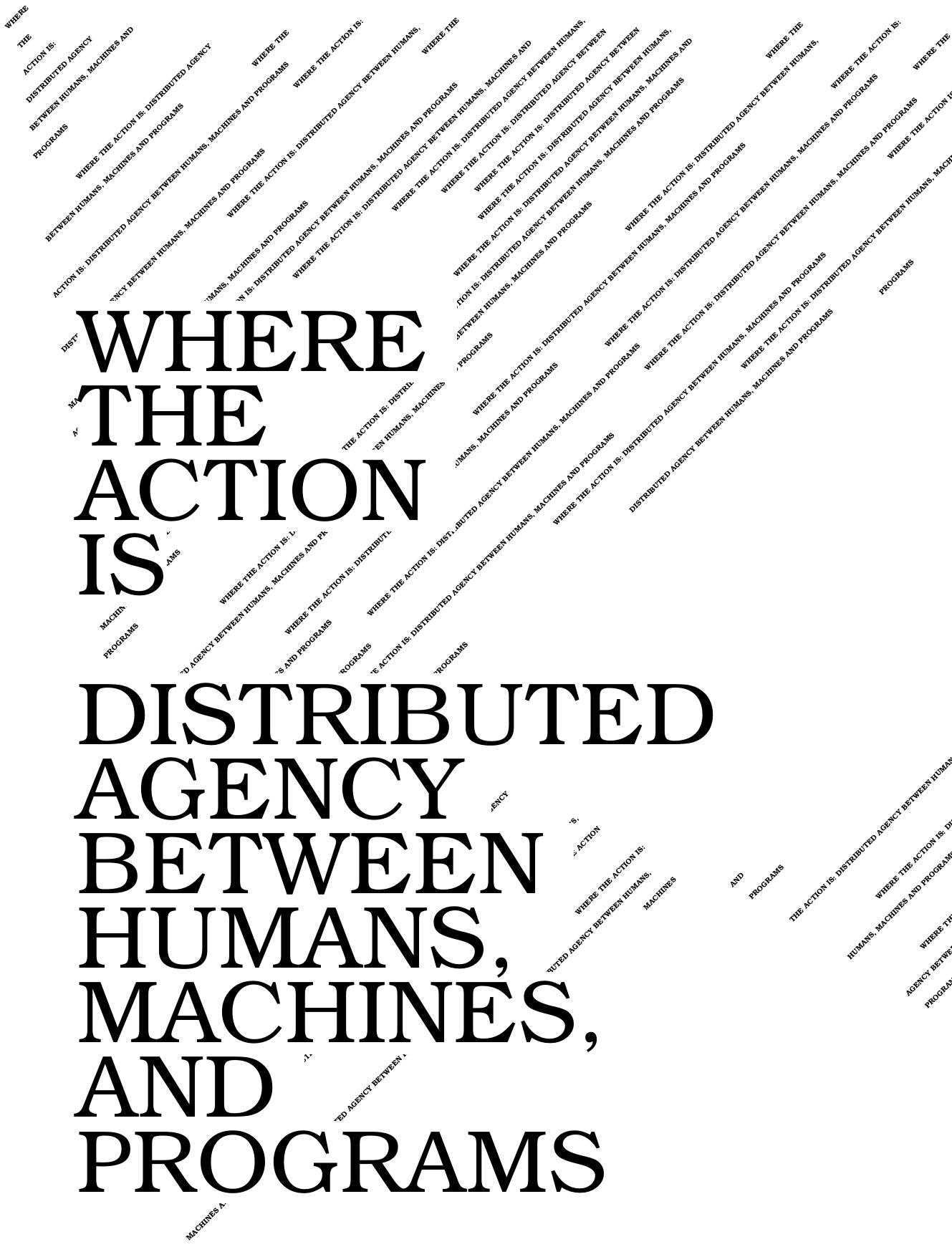

Werner Rammert 


\section{Distributed agency:}

\section{A concept beyond human action and technical means}

Usually, the action is where the humans are. Action means moving the body, making something, showing initiative, bringing about an alteration by force, and expressing oneself thereby. Action becomes particularly visible when there is unexpected reaction to something or resistance to somebody's will. In the humanities and social sciences, action is closely associated with the anthropological concepts of man the artist and tool-maker or the speaker and symbol-communicator. ${ }^{1}$ Human action - defined to be intentional and creative - is often sharply distinguished from animal behaviour, which is characterised as instinct-driven and only tool-using, and from machine operation that is described as a repetitive and pre-programmed activity. If we continue to define action by the demanding features of intentionality, rationality or reflexivity that are attributed to humans only, then - no wonder - all other uses of the term "action" in everyday life and actual technological developments would be only metaphors or even categorical mistakes. In this case we would miss and misunderstand the massive changes in intelligent machine design and interactive media use that open up Pandora's box filled with thousands of agents. These software or hardware agents equipped with belief, desire and intention algorithms are able to take part in manifold actions and even to change their action programs by case-based learning. Certainly, they are different from human actors, but they are also different from classical machines and media. Both features, their particular capacities of being active and interactive and their growing population in everyday gadgets and in the worldwide web of the internet, justify the undertaking which has been made in the following, to develop a more symmetrical and sophisticated concept of agency.

What are people talking about when they use the word 'action' in everyday life? Do the youngsters still mean good old human action, when they are acting in videogames inducing an avatar to follow and fight other creatures only by button-pushing? It is evident that button-pushing in this case is not the one single and simple instrumental action of fighting with swords, but one activity under many others: It activates a cascade of programs which themselves activate characters that show contingent action in a virtual action environment.

The players surely know the fundamental difference between the other human actors and the artificial agents in the game; but they are more interested in the interactivity and the particular high level of agency that they

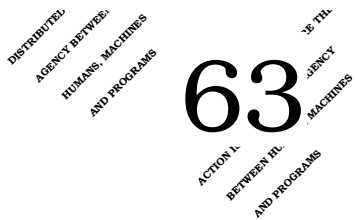


experience during their interaction with both kinds of partners or adversaries: the humans and the agents.

We learn something about the meaning of action when we listen to people talking about the genre of action films. They do not only mention the human actors who are in states of super-activity like running, jumping, or shooting and who are entangled in highly interactive situations like chasing one another or fighting with one another. Action includes more than human bodies in interaction. It is closely connected with the activities of high-speed vehicles, explosives and firing weapons as we know so well from James Bond films. Action of this special kind emerges from accelerated sequences of action of all kinds of acting units. The impression and fascination of action is finally produced by the many interactivities between the mixed agencies, not by the human interaction alone.

Actually, computer and media scientists use the vocabulary of human action when they describe the features of new technologies. Are software agents, for instance, really acting like human actors, when they ask the user for tasks, when they cooperate and compete with one another in the artificial society of agents, and when they assist persons in their daily actions of sorting out e-mails, searching for optimal traffic connections, looking for best prices, booking tickets and buying investment papers? Is it correct when interface designers speak about Human-Computer Interaction (HCI) and students of Distributed Artificial Intelligence call their programs 'agents' or 'multi-agent-systems' because they are constructed with the explicit intention to act like a person who is acting in the name of an other person? Action can be composed of different acts, and some can be delegated. Collective actions can be unified in a corporate actor like an organisation. They can be divided between principal and executive agents. ${ }^{2}$ If the actions are distributed between human actors and nonhuman agents, ${ }^{3}$ why should we not treat this 'hybrid constellation' as a particular kind of a collective actor?

Answering the main question 'where the action is' actually seems more complicated than before. The introductory considerations have alluded to four relevant changes in the sphere of human-technology relations that call for some conceptual revisions:

- The number of acting units and the kinds of action are increasing for the first time since modernity and enlightenment successfully diminished it by banning moving objects and talking trees, inviting nymphs and punishing gods, speaking oracles and helpful angels out of the sphere of action into the world of fetish and fiction.

2 Coleman 1990

3 Latour 1988 
- Instrumental actions between active people and passive objects are turned more and more into relations of interactivity between two heterogeneous sources of activities. The analysis and design of these relations require a more balancing approach of interactive contingency than a hierarchical one of instrumental causality.

- Actions are fragmented in many pieces and delegated to myriads of pro-active and cooperative agents on the back stage where they perform parts of the action by mimicking human agency and interpersonal interaction.

- Actions emerge out of complicated constellations that are made of a hybrid mix of agencies like people, machines, and programs and that are embedded in coherent frames of action. The analysis of these hybrid constellations is better done with a gradual concept of distributed agency than with the dual concept of human action and machine's operation.

In this paper it is argued that the advanced technologies take part in the course and constellation of human action and that they do this with real effects, not only metaphorically. The first part starts with the search for a useful concept of agency that enables the researcher to describe and classify all activities that contribute to the performance of an action. The concept shall include different levels of human agency as well as different levels of technologies in action (2). The following chapter treats the consequences that these activations of technologies have for the human-technology relation. If technologies change their role from passive means into agents and mediators, then the narrow concept of instrumental action should be replaced by a broader concept of inter-agency (3). This part of the paper culminates in the presentation of a gradual model of agency that can be used to describe and distinguish between different levels and grades of action without any regard to the ontological status of the acting unit, be it human-like or machine-like (4).

In the second part of the paper the question 'What is the adequate unit of action?' is answered. It starts with a thought experiment about the question: Who is really flying the Airbus? We learn from both views, the humanist's and the technologist's one, that what is usually called action, such as flying 240 tourists to Tenerife airport, consists of many distributed actions that have to be coordinated by social organisation or technical configuration (5). The concept of distributed agency is spelled out in three steps: It presupposes many loci of agency, not one actor (5.1). It declares the hybrid constellations made of the mixed human and material agencies to be the adequate research unit, and not solely the homogeneous social organisations or the technical configurations (5.2). Finally, a third mode of integration called 'framed interactivity' is 
elaborated that may emerge between the hierarchical mode of master-slaverelation and the open mode of autonomous systems (5.3).

\section{Technologies in action: From artifacts to agents}

Human action and technological operation belong to two different worlds: the realm of freedom and the realm of forces. Following Kant's definition, human action is characterised by its moral autonomy from external forces and laws. Although humans are subjected to these forces, they have the capacity (free will) to give themselves the rules of action that may become the general maxims for others, too. Referring to Reuleaux's definition, machines follow the very idea of forced movements. Heteronomy is the characteristic of von Foerster's "trivial machines" that are completely determined systems. ${ }^{4}$ The dichotomy of tool-maker and artifact is completed by the dichotomy of rule-making and rule-following.

This fundamental dichotomy may be helpful to divide between the ontological spheres of morality and causality. But it should not be applied to our questions of empirical changes and practical consequences. If we want to analyse the gradual changes of advanced technologies, the qualitative changes of the interaction between people and technologies, and even more, the re-configurations of the hybrid constellations from which action emerges, then we have to overcome this dual concept of action and operation. Thus we start with a symmetrical concept of agency that permits us to describe and classify what could be meant by the feature 'in action'. On this low level, we look for features of self-movement, activeness and self-acting.

How can we decide whether advanced technologies have changed and in which aspects? Let us take the five aspects that are often used in the engineering literature: technology as a motor/driver ("Motorik"), as an actuator ("Aktorik"), as a sensor system ("Sensorik"), as an information processor ("Informatik"), and as a communicator ("Telematik"). With respect to the aspect of motion, we can state that the gadgets and machines have gained higher degrees of self-movement: from one central stationary steam engine towards distributed systems of many engines powered by electric drive, from externally driven carts and coaches to self-driven vehicles, called automobiles. Under the aspect of acting and working, we make out a strong drift from crafted tools through mechanical machines to automatic systems.

The next three aspects seem to be of critical importance for the level of technologies which are subsumed under the label of "smart machines", "intelligent systems", "new electronic media", or "high technology". ${ }^{5}$ Regarding the

\footnotetext{
4 von Foerster 1985

5 Rammert 1992
} 
aspect of context-sensitivity, we actually realise a strong tendency away from systems that are completely blind to ones that are equipped with a feedback mechanism, all the way up to highly sensitive systems that are able to realise situations and to adapt their action to changing environments. The greatest steps in the direction of activating technical objects have been made with respect the aspect of information-processing: Looking backward, we reconstruct the movement as a loop from hard-wired tools and machines whose activity plans are incorporated in the design of the artifact, via flexible machines that are programmed by cards and records towards highly autonomous systems that strongly self-control their activities by nested systems of computer programs. Last, but not least, the aspect of communication between objects has emerged. Communication about the state of the machines' activity has been the task of people observing them at the work bench or in the office of the factory supervision. The direction is now inverted: The machines, the gadgets and even the products themselves observe the states, places and times of their actual activity and communicate them to people and also to one another via cable (Internet) or radio frequency (RFID).

\begin{tabular}{|c|c|c|c|}
\hline ASPECTS & FROM & CHANGE & TOWARDS \\
\hline Motor & stationary gadget & $>>$ & mobile agent \\
\hline Actuator & passive instrument & $>>$ & pro-active agent \\
\hline Sensor & blind machine & $>>$ & context-sensitive agent \\
\hline Processor & hard-wired artifact & $>>>$ & programmed agent \\
\hline Communicator & single apparatus & $>>$ & cooperating agent \\
\hline
\end{tabular}

Fig. 1. Aspects of technological change

The current advanced technologies show signs of increased self-activity within each aspect. As they are human-made technologies, they remain artifacts. However, they loose their passive, blind, and dumb character and gain the capacities to be pro-active, context-sensitive and co-operative. Insofar as the technical artifacts have been put into action by these changes, it is justifiable to define them as agents.

What are 'agents'? From a technological view, agents are particular computer programs. They are written with the intention that software agents can execute actions like human agents. This means that actions are delegated to them. The agents divide and delegate the action among other agents. They cooperate with one another, thereby moving, taking the initiative and addressing others. They coordinate the cooperation themselves and communicate the result of their activities to the human user. In a seminal text on intelligent agents, the main characteristics are presented as relative "autonomy", a par- 
ticular "reactivity" to the environment, "pro-activeness", and "sociability". ${ }^{6}$ From a sociological view, agents are persons who act in the name of a principal, e.g. the owner of an enterprise or as an informant of a party in a strategic spy game. ${ }^{7}$ The business and the secret service agent are bound to the general aims of the principal, but they are free to choose the adequate actions. Their actions are not blind executions of the principal's will. Agent-oriented programming and the design of architectures for multi-agent-systems follow this social concept of an agent and take over other mechanisms of society like cooperation, competition, trust or community in order to establish more flexible systems of distributed artificial intelligence. The up to now dominant design of a master-slave architecture is slowly being replaced by open systems of distributed and cooperating agents. The higher grade of activeness given to the software agents motivates the software engineers and the system designers to transfer those social and sociological concepts which have been proven as successful mechanisms of coordination. ${ }^{8}$

Technologies are changing on the level of technical systems, not only as concrete tools, machines, media, and sign processors. They show higher levels of complexity, they are more heterogeneously combined, and they are more complicatedly nested with one another. A review of the advanced technological and media systems reinforces the impression of a radical change in quality, not only in quantity and diffusion of technical objects. The Airbus is highly complex in a different way than a cathedral that is also made of millions of stones, glass pieces, and thousands of fixed relations between them, or than a Cadillac car in the fifties that is assembled out of thousands of exchangeable parts and has hundreds of variable relations between them. The cathedral and the Cadillac, however, combine heterogeneous materials and technologies, but the construction of an Airbus requires the integration of much more diverse technologies in an incomparable way. Especially, the embedding of so many different programmed physical and information systems in one plane produces the system's opacity that favours the interpretation of being confronted with an autonomous being. Stanley Kubrick clearly demonstrated this strange feeling in his "Odyssey in Space" when the computer system HAL, which was a part of the automated space ship, had to cope with contradictory rules in his program, then resisted human control and started to follow its own rules of action.

It is precisely to escape such fantasies of autonomous action on the one hand and the stubborn notion that technologies do not show any sign of agency on the other hand, that a more differentiated approach to the problem

6 Wooldridge/Jennings 1995

7 Goffman 1969

8 Schulz-Schaeffer 2002 
of technology in action should be developed. A scale with five levels of agency is presented here which may be seen as a first step on this route. The principle of its construction refers to the performance of technical objects and systems, not to their function. It also refers to the above-mentioned aspects and their interrelatedness. Examples from different technological domains are given for reasons of understanding. This scale is designed to raise awareness about different levels of agency and can be used for descriptive and classificatory reasons.

\begin{tabular}{|c|c|c|}
\hline LEVEL OF AGENCY & DESCRIPTION & EXAMPLES \\
\hline Passive & $\begin{array}{l}\text { Instruments completely } \\
\text { moved from outside }\end{array}$ & Hammer; Punching card \\
\hline
\end{tabular}

Semi-active

Re-active:

Pro-active:

Co-operative:
Apparatus with one aspect of self-acting

Systems with feedback loops

Systems with selfactivating programs

Distributed and selfcoordinating systems
Machine tool; Record-Player

Adaptive heating system

Car stabilisation;

Help agent

Mobile robots;

Smart Home

Fig. 2. Levels of agency for technical objects

It is not so easy to give examples that are typical of the particular level. The position in the scale depends on the precise description of the equipment and the connectedness between its parts. A brake can be a simple tool that functions mechanically. It can also be activated by a little motor; then it changes to the level of a semi-active hydraulic machine. When the brake is connected with a feed-back measurement instrument, it then operates on the level of re-activeness. Actual brake systems in the ICE or TGV trains are to be allocated on higher levels: They are pro-active, because they start their action themselves after having monitored and computed critical dates of inner and outer states. When there is also communication between the brake systems at the different wheels, then we can speak of a distributed and cooperative system. What can be learnt from this example? New insights cannot be gained from talking about agency on the first two or even three levels. It is completely sufficient to use the mechanical vocabulary of operation and determined movements. When the parts of a technical system, however, can behave not only in one pre-fixed way, but more flexibly, when the interaction with other parts or the interaction with the environment changes the behaviour, and when some parts actively search for new information to select their behaviour and even more to change their pre-given frame of action, then and 
only then does it make sense to use the vocabulary of agency and interaction in the world of objects.

\section{Types of Inter-Agency: From instrumentality to interactivity}

What makes it happen that a move, a behaviour or any other activity is recognised as a significant gesture or a meaningful action? How do we know whether a winking eye is only a body reflex or an intended signal to be willing to flirt? How do we know whether a flashing sign on our screen of our PC is a mechanical mistake, a routine recommendation to continue writing at this point or the triggered sign of an unexpected spy software? If one follows the social theory of pragmatism, the answer would be: One has to observe the sequence of three acts and relate them with one another as a circle of interaction. It is only at the end of this threefold interaction process that one can attribute the label causal effect, instinctive behaviour or meaningful action to the initial move.

Act 1: Winking eye $\gg>$ Act 2: Winking back $\gg>$ Act 3: Winking and laughing

Act 1: Winking eye $\gg>$ Act 2: Winking back $\gg>$ Act 3: Looking away

Act 1: Winking eye >> Act 2: Looking away ashamed $\gg>$ Act 3: Also looking away

These three sequences demonstrate that the meaning of the winkling eye in act 1 can only be ascertained after the next two acts: In the first line, the additional laughing completes the interaction circle and makes the first winking into a significant part of a social interaction called flirting. Act 3 in the second line constitutes the same meaning, but a different attitude to it, namely not being interested in flirting. The two consecutive acts of looking away in the third line seem to constitute a different meaning to the winking eye in act 1: It is an illness of nerves for which one does not want to stigmatise the person. What is important to note in the context of our argumentation, which is the central message of pragmatical interactionism is the following: The interactions observed and practised between the units of agency are what make critical differences and constitute the relevant meanings, not the individual act. 
This approach is usually applied to interpersonal interactions between human actors only, as in the case above. However, one can find some hints in the literature that the approach can also be transferred to relations between people and objects. ${ }^{9}$ For systematical reasons and for our particular purpose, three types of inter-agency should then be distinguished:

- Interaction between human actors,

- Intra-activity between technical agents, and

- Interactivity between people and objects.

Interpersonal interaction constitutes the social world of 'inter-subjectivity'. It is populated by human actors, expectancies and communications; it is structured by institutions, social systems and cultural meanings; it is the classical subject of the social sciences. Technical objects are principally excluded from this sphere of pure sociality; they figure either as neutral means for purposeful action, or as irritating objects from outside the society, or alternatively they are interpreted as mere carriers of meanings.

Intra-activity is quite an unusual term: In analogy to the relations between people it can be confined to the relations between objects, especially between technical agents. It constitutes the material world of 'inter-objectivity'. ${ }^{10}$ In so far as the objects show low levels of agency - according to our scale - and in so far as they are strongly coupled in linear, sequential or otherwise aggregated ways, one does not need to open the black box to study the internal operations. If, however, they display higher levels of agency and show more loosely coupled relations between the units, as in the cases of complex and high-risk systems or in cases of distributed and multi-agent systems, one should also follow the activities of the objects and describe their intra-activities. Otherwise one could not understand the differences which come up when people get into use relations with these kinds of technical systems. For it makes a difference whether people encounter an encapsulated system or a cooperating ensemble of agents, a hierarchical fixed order or an open network with case-based learning.

Interactivity is the term that is reserved for the cross-relations between people and objects. It belongs to the hybrid world of "interfaces", "humancomputer interaction" and "socio-technical systems". This boundary territory is widely occupied by the engineering sciences and their techno-morph approaches, such as the ergonomic models of the user as a body machine and a sensory mechanism, or the psychological models of "human factors" and "adaptive organism". It seems that the social sciences have given up this

$9 \quad$ McCarthy 1984

10 Latour 1996; Rammert 1998 
terrain at the limits of the social sphere, supposedly because they fear the contagious contact with "objectuality"11 and "materiality"12. Exceptions from this theoretical withdrawal can typically be discovered in the cultural media and science and technology studies. Bruno Latour has developed the most ambitious approach to re-present the things in the polity and to "re-assemble the social", including human and nonhumans. ${ }^{13}$ In my view, his actor-network methodology succeeds very well in bringing the "missing masses" 14 into the collective play, but the semiotics of actants ${ }^{15}$ cultivates a certain blindness towards observable actions and interactions and underrates processes of sense-making. Basing social theory in pragmatism may perhaps help to overcome such weaknesses. ${ }^{16}$

Pragmatism's social theory of interaction has been shown to be fruitful in explaining the production of social meaning by interpersonal interactions. This approach can also be used to analyse the relations of interactivity between people and physical objects. Georg Herbert Mead is famous for his comparative interaction analysis of two dogs fighting with one another and of two men boxing and faking against one another. ${ }^{17} \mathrm{He}$ has developed a not so well-known, but remarkable piece of theory about human interaction with physical objects: Children start to draw distinctions between different kinds of objects (own body, outside objects, moving, and living objects), after they have learnt the interpersonal role-model of social interaction. ${ }^{18}$ They analyse the activities and attributes of physical objects by taking over the role of them, as they have learned it by playing mother's or sister's role. Being heavy, flexible, moving, having an outer surface and an inner kernel, making noises and behaving in an unanticipated way, all these features of objects are experienced in children's play with stationary, mobile and interactive objects. Socialisation encompasses both processes: the interpersonal interaction between people, but also the interactivity with physical and symbolic objects.

This integrated view on inter-agency has implications for our own enterprise here, namely the inquiry into the changing character of advanced technologies and its consequences for human-technology relations. As long as technologies, such as simple tools and machines, can be characterised as passive or semi-active means, they are used in an instrumental mode: People

\footnotetext{
11 Knorr Cetina 1998

12 Pickering 1995

13 Latour 1994; Latour 2005

14 Latour 1992

15 Akrich/Latour 1992

16 Rammert 2007

17 Mead 1963

18 Joas 1989; Mead 1932
}

\section{2}


take and handle them to attain their goals at work or in other everyday life situations. The effective action of a tool or a machine is incorporated in its design, like the hammer's long shaft and heavy weight at the end or the engine's encapsulated explosion and the spark generated by the turn of the key. Therefore, the user can integrate these objects as mere instruments into his action. One can immediately begin using this kind of technology, and one can rely on the fixed function and the repetitious operations. One neither has to choose options out of a menu of options, nor is one involved in a dialogue with the machine. The only resistance or unexpected re-action of the technology would appear if the machine is out of order or the user is completely incompetent. When sociologists speak of "instrumental action", then they refer to this kind of unmediated instrumental relation between a man or a woman and a machine or a tool.

Gadgets and machines with higher complexity must be instructed before they start their efficient and useful activities. Simple versions of instruction can be already found on the classical tool machines: The craftsman instructed the machine by turning wheels and tuning measurement instruments. What started as a slow specialisation of instructing machines by Jacquard cards, paper stripes or record play-back was revolutionised by the invention of computer control and software programming from the 1940s onwards. The instruction of the machines' activities became a separate domain. In the long run, the devices were miniaturised and integrated into nearly every machine and gadget. They were turned into programmable machines, interactive media or smart objects. The instrumental use was changed: It was the beginning of an instructive-communicative relation between people and objects.

The rise of a third kind of relation can be observed when the machine asks back: Can I help you? Do you really want to delete the document? Please, tell me what makes you so sad? What looks like a dialogue between a woman at the reception and a guest, an assistant and his boss or a doctor and a client was the beginning of a new kind of relation between people talking to the machine on the one hand, and software programs that took over the roles of communicators, coordinators and agents of all kinds on the other hand. Weizenbaum's ELIZA program showed only marginal changes on the program's side. ${ }^{19}$ The program's reaction was restricted to take up some key words of the client's answer and to integrate them in a set of pre-given question sentences. Nowadays, the software agent technology has developed a much wider range of capacities to show higher levels of agency. The agents can deviate from the standard expectations. They can choose an activity out of a bundle of activities. They can assimilate their behaviour to the personal user. They can normalise their behaviour by drawing from statistics, and

19 Weizenbaum 1977 
they can change their behaviour by case-based reasoning. These features of agency force the user to conceive the relation as if an intelligent agent or partner were acting on the other side. Under these conditions of contingency and interagency, interactive-communicative relations are emerging.

Human-technology relations change when technologies are turned into more active agents and agencies. The instrumental relation that is typical for using tools in craft work and using machines like a tool is fading or only stage-managed as an illusion. The push on the button, the foot on the brake, and the click with the mouse trigger the activities between several agencies that more or less guide the machine, delegate the information-finding to Google's search algorithms, or confront the user with unexpected offerings and assistance because the profiling programs have made the user into an object. The user of this type of advanced technologies is neither the master of the machine nor the slave of the technological system, neither the sovereign of his action nor the victim of media's manipulation. A different concept is needed to decide the question of mastery or manipulation, case by case. The wider concept of inter-agency replaces the narrow one of instrumental use and of the perversion of means and goals. The more precisely both activities, the agency of objects and the inter-agency between objects, can be observed, the more the human-technology relation shows features of complex and contingent interactivity. Then the instrumental relation is only one particular case of an interrelationship. Relations of instructive and communicative interactivity are the other cases. They will dominate in the future, because nearly all kinds of technical objects will be equipped with programmed agency and will be made able to communicate with their environment. ${ }^{20}$

\section{A gradual model of agency: Analysing humans, machines, and programs in action}

The level of human agency is not necessarily always higher than the agency of machines and programs. Now we will bring together the two lines of argumentation that have been presented before separately. When people are in action, their level of agency is not always the highest possible one. They may act routinely, like handing over five 100 Euro notes at the bank counter. Or they may even do something without any intention, because they follow a hidden curriculum of a repressed desire. Reflexive action takes place when problems arise or irritations emerge in the course of action. Then people can switch from subconscious or routine action over to the next higher level of agency, searching for alternative courses of action or reflecting on the moral meaning. If one were to count the activities of people, only five percent could

20 Adelmann/Floerkemeier/Langheinrich 2006 
be classified as actions with reflected intentions. The rest follow practical reasons that could be mostly explained if asked for, or they follow everyday routines that often lack even practical reasons. ${ }^{21}$

When machines and programs are in action, their level of agency can be higher than usually perceived. Cash machines hand over the money like the human actor, at the same time examining the client's identity and credit line, varying the number of notes, signaling misuse, and stopping its activities. Even more, video surveillance cameras can be combined with pattern recognition software, interactive data-banks, and programs that process and mail notices of payment dues. They execute and coordinate actions a lot of police men on the street and employees in the offices would be needed for. Very simple dispositions are inscribed in this really existing London City law enforcement system. One can imagine multi-agent-systems to assist space flights or financial brokering whose software agents are equipped with even higher ranges of belief, desire and intention capacities in order to learn from reactions of other agents and from changing environments.

When the fundamental duality is to be be overcome of giving all of the action to the people and no parts of the action to the objects, then a concept of agency is required which also works with lower qualifications of the case of what an action is, on the one side. At the other side, it has to be more sophisticated about the question of what kind of action do we observe. Thus a gradual, three-level model of agency was developed, thereby referring to and distancing oneself from Giddens' three-level model of action and Latour's flattened concept of agency. ${ }^{22}$ Giddens distinguishes three levels of an action: a first one where a difference of state is produced, a second one where a difference of options is possible, and a third one where actors can give an explanation for their action if asked. ${ }^{23}$ We do not understand these levels as a necessary condition of action, but we interpret them as different levels of agency. We call these three levels "causality", "contingency", and "intentionality". Latour, however, pleads for a methodological and ontological symmetry and reduces all action to his flattened concept of agency. ${ }^{24}$ We share his antidualistic methodology, but we insist on levels and degrees of agency.

On the first level of causality, we start with a weak term of action. Agency of this kind means an efficient behaviour, a behaviour that exerts influence or has effects, as in the Latin term "agere" or in Latour's term "actant" or Callon's term "translation" 25 . Under the performative aspect on this level, it doesn't make any difference whether humans, machines or programs execute

21 Kaufmann 2008

22 Rammert/Schulz-Schaeffer 2002

23 Giddens 1984

24 Latour 1988; Latour 2005

25 Callon 1986 
the action. The money is handed over either by cash machines or by bank employees. The situation changes when greater irritations and more options come into play.

On the second level of contingency, the criterion of contingent action is required, which means the capacity to act in a different way and to choose between options. When the environment changes, the routine action program has to be changed and adapted to it, by people as well as by programs. Another possibility arises, when one's own action program is changed in such a way that its consequences are not immediately transparent and accountable for the others. When technologies reach this level of contingency, they cannot be used as immediate instruments any longer, and do not follow the paradigm of command and execution, as has been demonstrated in the previous chapter. Instrumentality is replaced by relations of interactivity. Dialogical inter-faces and internal user-modeling increase the action level. Interactive videogames create spaces of high virtual contingency ${ }^{26}$ that simulate human user's action. These technologies function like a Turing test: ${ }^{27}$ they make it nearly impossible to discriminate between human-enacted and computerenacted characters in the play.

On the third level of intentionality, the species of reflexive and intentional action is allocated. As long as intentionality is by definition ascribed to conscious and knowledgeable human actors only, this level is the domain of meaningful action that is oriented to the supposed meaningful action of other actors. Chessplaying programs cannot literally have the intention to win a game, but they can be constructed as if they had an intentional structure the philosopher Dennett calls this "from an intentional stance"28. Software agents cannot cooperate with others in a bodily manner and trust them under the explicit belief of augmenting their chances to reach a common goal. However, they can be equipped with an intentional vocabulary by which they really coordinate and communicate their activities as human actors do, with similar semantics. On this level, we plead against a substantial definition of action that excludes inquiries into agency. Instead we follow pragmatism, which means following all kinds of agencies and focussing on the observable practices in which cases the vocabulary of intentionality is used for the control or interpretation of activities of people as well as of technical objects. ${ }^{29}$

26 Esposito 1995

27 Turing 1950

28 Dennett 1987

29 Rammert/Schulz-Schaeffer 2002; Schulz-Schaeffer 2007

\section{6}


III.

Intentionality:

II.

Contingency:

I.

Causality:
>> up to guidance by complex semantics >> from ascription of simple dispositions

>> up to self-generation of actions > from selection of pre-selected options

>> up to permanent re-structuring of action >> from short-time irritation

Fig. 3. Levels and grades of agency ${ }^{30}$

This gradual and multi-level model of agency gives us the possibility to escape the dilemma of having to either reserve agency up to the humans or to flatten the concept of agency unnecessarily. Neither are we forced to claim that the activities of humans, machines and programs are substantially the same kind of behaviour. Nor do we have to stick to the conception that human action and technical operation are fundamentally different from one another. This gradual concept of agency opens up a wide range of possibilities to identify and to classify kinds and intensities of agency without regards to the substantial character of the unit that is in action. Thus the question of where the action is can be transformed into an empirical question.

\section{Distributed agency: The very idea}

The question of where the action is cannot be answered unless the answer to a second question has been clearly decided: What is the adequate unit of action? Conventionally, we suppose a single human actor to be the adequate unit of action: the philosopher who thinks and ergo knows that he exists, the employee who hands over the bank notes, the pilot who flies two hundred tourists to Tenerife airport, and so on. But let us look more precisely at the streams of actions from which an action arises. It arises as a distinct action, because it is sectioned off, retrospectively emphasised, and ascribed to a single unit, an actor or an author.

We would have never heard of Descartes' thought act if he had not written down his famous sentence with a pencil on paper. Even more, the working actions of dozens of printers were needed to distribute the phrase in hard-covered editions. Additionally, the teaching of hundreds of philosophy professors was necessary to diffuse the message under many thousands of

30 Rammert/Schulz-Schaeffer 2002 
students. Perhaps this thought act never took place as a single action at one place. Descartes was connected with a lot of thinkers whose arguments he received and whose papers he read. Perhaps the foundational thought act that is ascribed to him could have been discovered at many loci in that time, as if it would be very much "in the air". ${ }^{31}$ The act of writing interrupts this continuous chain of acts and turns it into the unique philosophical thought action that changed the world or at least the world view. The act of writing the sentence down by one single actor is emphasised, but both, the flux of thought acts before and the sequences of actions afterwards, such as printing, distributing, reading, teaching and learning, were put into brackets and neglected. It is an efficient strategy of teaching and tradition-building to attribute a thought act to one author because it reduces cognitive and social complexity. However, if we are doing research and inquire into the places, faces and activities where the action really is, we should follow all possible actors and agencies to the many loci of agency. ${ }^{32}$

\subsection{Distributed agency I: From a single actor to many loci of agency}

A thought experiment will be used to introduce the second part of the paper: Let us answer precisely the question: Who or what is acting in the case of flying the tourists to Tenerife?

PEOPLE

Pilot?

Co-pilot?

Radio operator?

Flight-controller?

Tourist office?

Airline company?

Aviation industry?
MACHINES

Jet engine?

Elevator, Rudder?

Radio equipment?

Radar unit?

Booking machine?

Aviation technology?

Air traffic system?

\section{PROGRAMS}

Auto-pilot software?

Navigation card and system?

Radio signals and codes?

Radar screening?

Reservation software?

Technological R\&D plans?

Roadmaps for infrastructure?

Fig. 4. List of actors and agencies in the flight case

31 Merton 1957

32 Latour 1987; Rammert/Schubert 2006; Schubert 2007 
Humanists and social scientists focus on the people's side in the list. Their first and most plausible answer will be that the human pilot is the acting unit that flies the tourists to Tenerife. He is conscious of the goal, the methods and instruments. He reflects on possible interventions into the path of the aircraft and deviations. Finally, he can be made responsible for the flight because he has the power of command and control. But a first uncertainty appears when one is confronted with the question: Doesn't the captain have at least one radio-operator at his side? We know from some cases of accidents that the communicative actions between pilot and co-pilot or between pilot and flight-controller have been critical for the flight action: the consequence can entail escaping a collision or not. So we learn that agency can be divided between several human actors. The acting unit, then, is either the team on board or the locally dispersed assembly of people on board and at several control centres on earth. A further question raises other doubts about the single heroic actor: Does the captain or this group of navigators and controller really plan the flight action? No, it was the air line which planned the route, the time and the final departure. It needs more than 200 paying passengers so that the action can take place. In comparison to this powerful principal agent the other actors fall back in the role of executing agents. The company is the so-called collective actor which plans, decides, and controls the flight action to Tenerife. In sum, four different units of action can be distinguished on the people's side: a single human actor, a social group or team, a dispersed association of people being in interaction by a division of work, and a collective actor that coordinates activities towards a goal. Certainly, human agency is multiplied, divided, distributed, and connected.

Encouraged by our gradual concept of agency, one may dare to insist on a more precise answer to the question of what actors and other agencies contribute to the flight action. Engineers and scientists probably would emphasise the role of machines and programs. Their first and most plausible answer would be: No pilot and no flight without up-currents or artificial drivers, like propellers or jet propulsion! Elevators and rudders give the air plane the direction, and the radio and radar equipment enables the plane to find its position and to correct its route. As we have discussed earlier, the agency on this low level of causality doesn't really add new explanatory power. But the situation changes completely when these machine technologies and communication media are in close intra-activity with the agencies that are enlisted on the programs' side. For the most time of the flight, the flight action and the many sub-actions are delegated to the auto-pilot. This is a combination of many different software programs that are continually measuring, monitoring, and computing, but also actively correcting the height, tempo and direction of the flight. The automatic landing system sometimes even restricts the human pilot from intervening into the action. In sum, the unit of technical 
agency is constantly changing and growing towards a highly combinatory and relatively autonomous technological system. It starts with wings and rudders. It develops into an aggregated technological system integrating many sub-units such as propulsion, navigation, and communication systems. A qualitative shift in the level of agency is achieved at the end, when advanced computer programs take over the planning, control and navigation activities, especially their intelligent coordination, and even more when the flying plane itself is turned into one agent in a more extended and self-regulating air traffic system.

In the end, we see that it is not so easy to define a human and, in particular, a social action. Philosophical and sociological textbooks may help to think about the criteria. The authors usually start with a concept of action that is isolated from the stream of other actions and that is idealised in a certain way. The "ego" is the unit that creates changes, and chooses and defines the situation, like God the creator. One can call this concept of action "agency ex nihilo" and contrast it with an alternative one, "agency in medias res" 33 , that reconstructs action out of the many activities before and around the focused action. Flying 200 tourists to Tenerife is not the instrumental action of a pilot navigating the plane to Tenerife airport. It is one activity that is combined with other activities of controlling and communication. It is additionally integrated in the commercial activities of an airline company. Finally, it is also nested in the activities of a highly complex organised system within air traffic, the aviation sector and the tourist industry. Looking at the activities from this perspective, one discovers many loci of agency instead of one single actor. One can reconstruct the flight action as the commercial action of a collective actor or even a network of organisations ${ }^{34}$ which hire people, invest in new planes, lobby for public support, advertise cheap charter flights, and organise the flight route.

Looking at the technical side of the list, the talk of gadgets and machines as simple means of action underrates both the complexity of aggregated technical systems and the self-activeness of programmed and nested systems. The collection of many devices and the compilation of different types of technologies cannot be handled like bigger tool-boxes with an increasing number of instruments in it. These interrelated parts build highly complex systems, with many planned intra-activities and some unforeseen interferences, ${ }^{35}$ so that they lose the clear transparency of an instrument and require strategies of interactivity for their control. The combination of nearly all parts with computing and communication capacities converts them into pro-active

\footnotetext{
33 Fuller 1994

34 Teubner 2003

35 Perrow 1986
} 
agents that often are connected in relatively autonomous systems on a higher level, like the automatic landing system or the internet-based reservation and booking system. As the advanced technologies mostly simulate human actions, the different tasks, roles and competencies and actually also the social mechanisms of coordination, it makes sense to describe these activities and intra-activities with the vocabulary of action and inter-agency. It is the adequate way to discover the many loci of technological agency.

\subsection{Distributed agency II: From homogeneous agency to hybrid constellations}

In the predominant dualist tradition of thought, the social and cultural world of human action, and the material and artificial world of technological operation are separated from one another. On the one hand, social scientists focus on the motives and expectations of people, such as pilots and flight ticket sellers, and on the kinds of social organisation. They reconstruct a homogeneous world of symbolic interaction and communication purified of physical objects. On the other hand, engineering scientists are preoccupied with questions of setting something going, such as air planes or software programs, and of improving the effectiveness or safety of technological configurations. They construct a homogenous world of forced movements and functioning technological systems purified from social interests and human users. Facing the growing interrelatedness of problems of nature and of society, such as man-made climate change or artificial stem cell growing, and facing the co-construction of socio-technical systems made of people, machines and programs, one may, however, ask whether a non-dualist conceptual approach could help to make these hybrid constellations a sound subject of research.

At the borders between the two academic cultures, we already observe regular border traffic and even conceptual bridge-building. From research in technology and organisations, approaches are being pushed forward that respond to the strong interdependency between the material and the social, such as the Tavistock approach of socio-technical systems, ${ }^{36}$ the concept of large technical systems consisting of people, organisations, material and symbolic artifacts, ${ }^{37}$ and comparative analysis of high risk systems screening them along aspects of complexity and interaction between human and nonhuman elements. ${ }^{38}$ The most influential approaches took research in science and technology as their point of departure. Some researchers of this area

36 Trist 1981

37 Hughes 1987; Mayntz/Schneider 1988

38 Perrow 1984 
argue against bridging and proposed a radical change of perspective, ${ }^{39}$ such as particularly the adherents of the ANT approach, but also of the concepts of "objectuality"40, "socio-technical agency"41, and of "material agency" Research in media and culture is actually a growing third branch where hybrid constellations are the new subjects, like being a "cyborg", "technoscience" or living in "virtual life". ${ }^{43}$ Bridge-building and trans-disciplinary concept-development can be also observed at the science side of the border. Particularly the engineering sciences cross the border and take up concepts of the humanities and the social sciences. Interface designers integrate psychological concepts of cognition and sociological concepts of routine-building and role specialisation. Designers of software agents apply philosophical concepts of mind, belief and intention. And the architectural designers of multi-agent systems use sociological concepts of trust, contractual, and market relations.

From the dualist point of view it makes sense to keep the two territories separated. A lot of arguments can be mobilised for this decision, such as the ontological differences between people and machines, ${ }^{44}$ the epistemological differences between the disciplines, the institutional differences between social organisations and technical configurations and so on. But these differences lose their relevance under certain conditions: When human actions, machine operations and programmed activities are so closely knit together that they form a "seamless web" 45 , then it makes sense to analyse this hybrid constellation as a heterogeneous network of activities and interactivities. When a human action such as flying an Airbus or searching for a certain piece of information in hundreds of libraries, millions of books, and trillions of files can only be executed with the assistance and intervention of hundreds of other agencies, then it is urgent to develop a concept of agency that acknowledges all these agencies, though they are heterogeneous in substance. And finally, when programmed machine operation is developed such that it should execute delegated actions under conditions of contingency, and when it is implemented in open systems that are constructed by the interactions between the software agents, then one should integrate these agencies into the framework of analysis. Therefore a concept of distributed agency is argued for here only under these conditions of advanced technologies and instituted hybrid constellations.

\footnotetext{
39 Callon/Latour 1992; Collins/Yearley 1992

40 Knorr Cetina 1998

41 Girard/Stark 2007; Preda 2006

42 Pickering 1993

43 Haraway 1991; Haraway 1997; Ihde/Seliger 2003; Turkle 1995

44 Collins/Kusch 1998

45 Hughes 1986
} 
Coming back to our flight action example, the answer to the question of what the adequate unit of action is can now be given: It is the hybrid constellation of people, machines, and programs. It is the mode in which the agencies of the heterogeneous instances are distributed and connected with one another and the level of agency that is given to them in certain situations. It is neither the single or the collective human actor, nor the technical artifact alone, nor the combined technical system. It is the mixed ensemble made of all elements on both sides of the border. One can call it a collective agency, alluding to the term "collective actor". This collective is constituted by the distributed activities of heterogeneous units in comparison to what is referred to by the other term, which is built out of the homogeneous stuff of human actions.

\subsection{Distributed Agency III: From hierarchy to framed interactivity}

Two modes can be distinguished in which actions can be divided and integrated: a hierarchical mode in which specialised activities are strongly integrated, and an interactive mode in which distributed modal units are weakly coupled. In the sociology of organising, they are often referred to when distinctions are made between bureaucratic and organic models or between strongly or weakly coupled systems. ${ }^{46}$ Observing complex organisations, one learns very quickly that hierarchical integration is only the most effective mode for divisions with fixed inputs, routine processing and stable environment, like the mass-production of things. Units that are confronted with changing inputs, many variations in the process and dynamic environments require a more interactive, flexible and open mode of organising, like $R \& D$ departments or creative industries. Most of the modern organisations show a mix of both modes of integration, mechanising the routine parts and learning by interactivity with the environment.

It was taken for granted up to now that the hierarchical mode was the only and the best way to specialise and integrate technologies. It was the paradigm for the first machine made of the forced movements of people working based upon a division of labour to build the pyramids, and also for the ongoing process we call mechanisation. ${ }^{47}$ Technologies are defined by their capacity to force different activities into a mechanical form that is reliable, accountable and usable as a mean to solve particular problems in an effective and expected way. ${ }^{48}$ Tasks are divided between many specialised parts

46 Perrow 1986; Weick 1976

47 Giedion 1948

48 Rammert 2001 
and integrated by linear chains of operations and hierarchical schemes of processing. However, this dominant mode and its supposed universality are now being challenged. Some technical configurations and socio-technical constellations can be observed that are integrated in a different mode that resembles the above mentioned interactive mode.

One can already observe small deviations from the strong mechanical mode when looking at the feed-back loops of cybernetic systems. The sandwich architecture of the computer also shows a loosening of point-to-point determination between its physical machine level and the logical level and the program language level. ${ }^{49} \mathrm{~A}$ further milestone on the path of breaking the linearity was the concept of "distributed computing" 50 . It started with the simple problem of distributing computing time, but gained its momentum when a new generation of software programs were developed that used fuzzy logic, distributed artificial intelligence, agent-oriented programming, and models of socionics in order to admit distributed activities and parallel processes. Particularly in social computing ${ }^{51}$ and in socionics ${ }^{52}$, many modes of interactive integration were developed that were in opposition to the hierarchical mode.

Another milestone was the development of the concept of "distributed cognition"53. The psychologist Hutchins criticises the dominant model of individual problem-solving in the cognitive sciences that supplies the artificial intelligence community with a construction plan. It presupposes separated and functional specialised activities that can be easily aggregated. Also being an ethnographer, Hutchins observed the techniques of navigation "in the wild" and "in medias res": He studied precisely how the Polynesian longdistance sailors performed navigation in the wide Pacific ocean though they had no sophisticated nautical instruments, and how a navigation team on a warship maneuvered their long ship into the small harbor entrance of San Diego though its nautical system was damaged. In this way he discovered a mode of self-organised integration between distributed processes of cognitive activities. The cognitive action of positioning was organised as a distributed process that was performed by some people with different practices, natural objects and technical instruments. The critical point for our argumentation here is his observation that these distributed processes did not require any planning, functional specialisation or hierarchical integration. Their mode of integration was described as a natural process of loose coupling, overlapping

49 Winograd/Flores 1986

50 Rumelhart/McClelland 1986

51 Hewitt 1977; Star 1989

52 Malsch 2001; Meister et al. 2007; Rammert 1998

53 Hutchins 1996 
activities, experimental adaptation, and a step-by-step stabilisation of a common frame for the interactions. ${ }^{54}$

The concept of "distributed agency" that is presented in this paper follows the lines that were started by those concepts of "distributed computing" and "distributed cognition". The first step towards constructing this concept of distributed agency has been to demonstrate that human action is distributed between many loci and instances that plan, control, and execute the activities. Distributed action means that someone searches for significant marks, someone else measures the angles, a third person plots by drawing a line, and others count, communicate and correct the data. All these interactions between them constitute an observable unit of action called navigation. This kind of distribution can also be transferred to computer operations. The action of sending a message to a certain person can be broken down into many activities at different places, such as encoding, packaging, addressing, transporting, and reading TCP/IP protocols at the $\mathrm{PC}$, at the server, at the local area network, or at one of the knots of the worldwide web.

The second step has been to cross the Rubicon between the two homogenous spheres of human action and technological operation: distributed agency then refers to hybrid constellations made of heterogeneous units of agency. Moving objects such as the sun and the currents of water, measuring instruments, counting tables, and carved records participated in the action of navigation. As we have argued before, objects participate more actively and on a higher level of agency when the nautical pilot program and the automatic navigation system are in action and in close intra-activity with one another.

The third step now emphasises the two modes of integration. They differ in how the units are divided, how they are processed, and how they are connected with one another. The dominant hierarchical mode of integration prolongs the traditional line that allows us to treat even complex technologies and hybrid constellations as reliable means and robust mechanisms. The mode of framed interactivity is rarely implemented because it deviates from the well-known and trusted master-slave relation. The technological units are given more freedom of choice and higher levels of agency in order to enrich their capacity of assistance and to strengthen their role as relatively autonomous agents.

54 Hutchins 1998 


\section{Type of}

Differentiation

\section{Type of \\ Organisation}

Type of

Connection
Division of work

Functional specialisation

Mechanical

Bureaucratic

Linear sequences

Strongly coupled

Fixed and general rules

Pre-Programmed
Distributed activities

Fragmented units

Organic

Open System

Parallel processes

Loosely coupled

Flexible, situated, and specific rules

Framed Self-adaptation

\section{Fig. 5. Two modes of integration}

Though the mode of framed interactivity has rarely been implemented up to now, this mode may become a new paradigm for the design of future constellations. It is currently sought after in many different places: in laboratories of distributed intelligence, in research and development clusters on robotics, man-machine interfaces and new media design, as well as in the studios of interactive artists, in the media labs of the entertainment industry, and at the software benches of videogame developers. This mode of framed interactivity will get its chance to be diffused when the next generation of technologies is consciously designed and implemented from the perspective of distributed agency, when the frames of heterogeneous agencies are balanced and tuned to each other, and when a new generation of users is coming up that is used to the new experiences with interactivity. 


\section{References}

Adelmann, Robert/Langheinrich, Marc/Floerkemeier, Christian (2006): »Toolkit for Bar Code Recognition and Resolving on Camera Phones - Jump Starting the Internet of Things«. In: Christian Hochberger/Rüdiger Liskowsky (Eds.), Informatik 2006 Informatik für Menschen, Band 2, Beiträge der 36. Jahrestagung der Gesellschaft für Informatik e.V. (GI), 2.-6. Oktober 2006 in Dresden, 366-373.

Akrich, Madeline/Latour, Bruno (1992): »A Summary of a Convenient Vocabulary for the Semiotics of Humans and Nonhuman Assemblies«. In: Wiebe E. Bijker / John Law (Eds.), Shaping Technology - Building Society, Cambridge: MIT Press, 259-264.

Callon, Michel (1986): »Some Elements of a Sociology of Translation: Domestication of the Scallops and the Fishermen of St. Brieux Bay«. In: John Law (Ed.), Power, Action, and Belief, London: Routledge, 196-229.

Callon, Michel/Latour, Bruno (1992): »Do not throw out the baby with the Bath school! A reply to Collins and Yearley«. In: Andrew Pickering (Ed.), Science as Practice and Culture, Chicago: University of Chicago Press, 343-368.

Coleman, John S. (1990): Foundations of Social Theory, Cambridge: The Belknap Press of Harvard University Press.

Collins, Harry M./Yearley, Steven (1992): „Epistemological Chicken«. In: Andrew Pickering (Ed.), Science as Practice and Culture, Chicago: University of Chicago Press, 301-326.

Collins, Harry M./Kusch, Martin (1998): The Shape of Actions. What Humans and Machines Can Do, Cambridge: MIT Press.

Dennett, Daniel (1987): The Intentional Stance, Cambridge, MA: MIT Press.

Esposito, Elena (1995): »llusion und Virtualität: Kommunikative Veränderungen der Fiktion«. In: Werner Rammert (Ed.), Soziologie und künstliche Intelligenz. Produkte und Probleme einer Hochtechnologie, Frankfurt a.M./New York: Campus, 187-216.

Foerster, Heinz von (1985): Sicht und Einsicht. Versuche zu einer operativen Erkenntnistheorie, Braunschweig: Vieweg.

Fuller, Steve (1994): »Making Agency Count. A Brief Foray into the Foundation of Social Theory«. American Behavioral Scientist 37, 741-753.

Giddens, Anthony (1984): The Constitution of Society: Outline of the Theory of Structuration, Cambridge: Polity Press.

Giedion, Siegfried (1948): Mechanization takes command: a contribution to anonymous history, New York: Oxford University Press.

Girard, Monique/Stark, David (2007): »Socio-technologies of Assembly: Sense-Making and Demonstration in Rebuilding Lower Manhattan«. In: David Lazar/Viktor MayerSchoenberger (Eds.), Governance and Information: The Rewiring of Governance and Deliberation in the 21st Century, Oxford: Oxford University Press, 145-176.

Goffman, Erving (1969): Strategic Interaction, New York: Routledge.

Haraway, Donna J. (1991): Simians, Cyborgs, and Women: The Reinvention of Nature, New York: Routledge. 
Haraway, Donna J. (1997): Modest_Witness@Second_Millennium. FemaleMan_Meets_

OncoMouse. Feminism and Technoscience, New York: Routledge.

Hewitt, Carl E. (1977) »Viewing Control Structures as Patterns of Passing Messages«. Artificial Intelligence 8, 323-364.

Hughes, Thomas P. (1986): »The Seamless Web: Technology, Science, Etcetera, Etcetera«. Social Studies 16, 281-292.

Hughes, Thomas P. (1987): »The Evolution of Large Technological Systems«. In: Wiebe E. Bijker/Thomas P. Hughes/Trevor. J. Pinch (Eds.), The Social Construction of Technological Systems, Cambridge: MIT Press, 51-82.

Hughes, Thomas P. (1998): »Learning to Navigate. Understanding Practice. Perspectives on Activity and Context«. In: Seth Chaiklin/Jean Lave (Eds.), Unterstanding Practice. Perspectives on Activity and Context, Cambridge: Cambridge University Press, 35-63.

Hutchins, Edwin (1996): Cognition in the Wild, Cambridge: MIT Press.

Ihde, Don/Seliger, Evan (2003): Chasing Technoscience: Matrix for Materiality, Bloomington: Indiana University Press.

Joas, Hans (1989): Praktische Intersubjektivität. Die Entwicklung des Werkes von G. H. Mead, Frankfurt a.M.: Suhrkamp.

Kaufmann, Jean-Claude (2008): Was sich liebt, das nervt sich, Konstanz: UVK-Verlag.

Knorr Cetina, Karin (1998): "Sozialität mit Objekten. Soziale Beziehungen in posttraditionalen Wissensgesellschaften«. In: Werner Rammert (Ed.), Technik und Sozialtheorie, Frankfurt a.M.: Campus, 83-120.

Latour, Bruno (1987): Science in Action: How to Follow Scientists and Engineers Through Society, Cambridge: Harvard University Press.

Latour, Bruno (1988): »Mixing Humans and Nonhumans together: The Sociology of a Door-Closer«. Social Problems 35, 298-310.

Latour, Bruno (1992): »Where are the Missing Masses? The Sociology of a Few Mundane Artifacts $«$. In: Wiebe E. Bijker/John Law (Eds.), Shaping Technology - Building Society, Cambridge: MIT Press, 225-258.

Latour, Bruno (1994): On Technical Mediation: The Messenger Lectures on the Evolution of Civilization«. Common Knowledge 3, 29-64.

Latour, Bruno (1996): »On Interobjectivity«. Mind, Culture, and Activity 3, 228-245.

Latour, Bruno (2005): Reassembling the Social. An Introduction to Actor-NetworkTheory, Oxford: Oxford University Press.

Leroi-Gourhan, André (1980): Hand und Wort. Die Evolution von Technik, Sprache und Kunst, Frankfurt a.M.: Suhrkamp.

Malsch, Thomas (2001): »Naming the Unnamable: Socionics or the Sociological Turn of/ to Distributed Artificial Intelligence $\ll$. Autonomous Agents and Multi-Agent Systems 4, 155-186.

Mayntz, Renate/Schneider, Volker (1988): "The Dynamics of System Development in Comparative Perspective: Interactive Videotext in Germany, France, and Britain«. 
In: Renate Mayntz/Thomas P. Hughes (Eds.), The Development of Large Technical Systems, Bolder: Westview Press, 263-298.

McCarthy, E. Doyle (1984): »Toward a Sociology of the Physical World: Mead on Physical Objects«. Studies in Symbolic Interactionism 8, 105-121.

Mead, George H. (1932): The Philosophy of the Present, Edited by Arthur E. Murphy, La Salle: Open Court.

Mead, George H. (1963): Mind, self and society, Chicago: University of Chicago Press.

Meister, Martin/Schröter, Kay/Urbig, Diemo/Lettkemann, Eric/Burkhard, HansDieter / Rammert, Werner (2007): »Construction and Evaluation of Social Agents in Hybrid Settings: Approach and Experimental Results of the INKA Project«. Journal of Artificial Societies and Social Simulation 10 [electronic journal], <http://jasss.soc. surrey.ac.uk/JASSS.html> (last access: July 2008).

Merton, Robert K. (1957): »Priorities in Scientific Discovery: A Chapter in the Sociology of Science«. American Sociological Review 22, 635-659.

Perrow, Charles (1984): Normal Accidents: Living With High Risk Technologies, Princeton: Princeton University Press.

Perrow, Charles (1986): Complex Organizations. A Critical Essay, New York: Random House.

Pickering, Andrew (1993): »The Mangle of Practice: Agency and Emergence in the Sociology of Science«. American Journal of Sociology 99, 559-589.

Pickering, Andrew (1995): The Mangle of Practice: Time, Agency and Science, Chicago: University of Chicago Press.

Preda, Alex (2006): »Socio-technical Agency in Financial Markets: The Case of the Stock Ticker«. Social Studies of Science 26, 753-782.

Rammert, Werner (1992): »From Mechanical Engineering to Information Engineering: Phenomenology and the Social Roots of an Emerging Type of Technology«. In: Meinolf Dierkes/Ute Hoffmann (Eds.), New Technology at the Outset. Social Forces in the Shaping of Technological Innovations, Frankfurt a.M.: Campus/Bolder: Westview, 193-205.

Rammert, Werner (1998): „Giddens und die Gesellschaft der Heinzelmännchen. Zur Soziologie technischer Agenten und Systeme Verteilter Künstlicher Intelligenz«. In: Thomas Malsch (Ed.), Sozionik. Soziologische Ansichten über künstliche Sozialität, Berlin: Sigma, 91-128.

Rammert, Werner (2001): »Relations that Constitute Technology and Media that Make a Difference: Toward a Social Pragmatic Theory of Technicization«. In: Hans Lenk/ Matthias Maring (Eds.), Advances and Problems in the Philosophy of Technology, Münster: Lit Verlag, 271-290.

Rammert, Werner (2007): Technik - Handeln - Wissen. Zu einer pragmatistischen Technik- und Sozialtheorie, Wiesbaden: VS Verlag.

Rammert, Werner/Schulz-Schaeffer, Ingo (2002): »Technik und Handeln - Wenn soziales Handeln sich auf menschliches Verhalten und technische Artefakte verteilt«. 
In: Werner Rammert/Ingo Schulz-Schaeffer (Eds.), Können Maschinen handeln? Frankfurt a.M.: Campus, 11-64.

Rammert, Werner/Schubert, Cornelius (2006): Technografie. Zur Mikrosoziologie der Technik, Frankfurt a.M.: Campus.

Rumelhart, David/McClelland, James (1986): Parallel Distributed Processing. Vol. I. Foundations, Cambridge: MIT Press.

Schubert, Cornelius (2007): »Risk and safety in the operating theatre. An ethnographic study of socio-technical practices«. In: Regula V. Burri/Joseph Dumit (Eds.), Biomedicine as culture. Instrumental practices, technoscientific knowledge, and new modes of life, London: Routledge, 123-138.

Schulz-Schaeffer, Ingo (2002): »Innovation durch Konzeptübertragung. Der Rückgriff auf Bekanntes bei der Erzeugung technischer Neuerungen am Beispiel der Multiagentensystem-Forschung«. Zeitschrift für Soziologie 31, 232-251.

Schulz-Schaeffer, Ingo (2007): Zugeschriebene Handlungen. Ein Beitrag zur Theorie sozialen Handelns, Weilersvist: Velbrück.

Star, Susan L. (1989): »The Structure of Ill-Structured Solutions: Boundary Objects and Heterogeneous Distributed Problem Solving«. In: Michael Huhns/Les Gasser (Eds.), Distributed Artificial Intelligence, Menlo Park: Morgan Kauffman, 37-54.

Teubner, Gunther (2002): »Hybrid Laws. Constitutionalizing Private Governance Networks«. In: Robert Kagan/Kenneth Winston (Eds.), Legality and Community, Berkeley: California University Press.

Trist, Eric (1981): The Evolution of Socio-Technical Systems: A conceptual framework and an action research program, Toronto: Ontario Quality of Working Life Centre.

Turing, Alan (1950): »Computing Machinery and Intelligence«. Mind 59, 433-460.

Turkle, Sherry (1995): Life on the Screen. Identity in the Age of Internet, New York: Simon \& Schuster.

Weick, Karl (1976): »Educational Organizations as Loosely Coupled Systems«. Administrative Science Quarterly 21, 1-19.

Weizenbaum, Joseph (1977): Die Macht der Computer und die Ohnmacht der Vernunft, Frankfurt a.M.: Suhrkamp.

Winograd, Terry/Flores, Fernando (1986): Understanding Computers and Cognition. A New Foundation for Design, Reading: Addison-Wesley.

Wooldridge, Michael/Jennings, Nicholas (1995): »Intelligent Agents: Theory and Practice«. The Knowledge Engineering Review 10, 115-152. 


\section{Acknowledgements}

I thank Cornelius Schubert for critical comments and Kristina Brandt for technical assistance. This paper would not have been written without the DFG-support "Technik in Aktion" and the invitation to the $\mathrm{ZiF}$ research group "Science in the Context of Application" at the University of Bielefeld. 
Jürgen Habermas' essay "Arbeit und Interaktion" first appeared in an anthology in 1967. One year later, it was put together with four more of his texts and re-published under the title "Technik und Wissenschaft als 'Ideologie". ${ }^{1}$

When I first read it in 1972, it was because of the word "labor" in its title. I don't remember a thing from the fist reading, and the habitual underlinings and marginal remarks on the pages of the book don't indicate from when they are. I guess I put the book away on my bookshelf where it remained for more than a decade, perhaps untouched. When I read it the second time, probably in the 1980s, it was more or less by accident. I was browsing my bookshelves in search of something by Marcuse, when out of some intuitive reason I grabbed the thin Habermas volume and only then became aware of the other word, "interaction", in the title "labor and interaction". At the time (in the 1980s) I was teaching a four semester cycle in computer graphics whose specific topics were foundations, geometric modeling, graphic rendering, and techniques of interaction. Clearly, my bookshelf discovery forced me to immediately sit down and study what Habermas had to say on interaction. Why was he using the term?

Imagine the timely context of the situation! Charles P. Snow had delivered his "The two cultures and the scientific revolution" in Cambridge in 1959. I had then been a student of mathematics at the University of Stuttgart. A year before, during a two month internship at IBM in their Böblingen Computing Centre, I had had my first encounter with a computer. Max Bense, the provocative philosopher at Stuttgart, had surely referred to Snow's talk and concept of two cultures. I found myself caught in a great melting pot: studying mathematics, the queen of all mental efforts, experiencing the grandeur and joy of strict axiomatics, formal concepts, theorems, and proves. From this comfortable centre to the left were Bense's thrilling lectures about aesthetics, ontology and, particularly, semiotics; to the right was theoretical and experimental physics, or the theory of electrical engineering, and more.

What a time, what a storm! We were a group of friends, trying to understand what the engineering types told us as well as what came from those in the humanities. We felt more and more at home in mathematics and, soon enough, in its rather trivial offspring, computing. But now we were confronted with Snow's claim that no communication was possible across the boundaries between the two types of disciplines we liked so much, because they were both exciting in their own way: the scientific and the literary cultures. It

1 The German original I am working with is Habermas 1969. It contains on pp. 9-46 the essay "Arbeit und Interaktion. Bemerkungen zu Hegels Jenenser 'Philosophie des Geistes". It is available in English as "Labor and interaction: comment on Hegel's Jena 'Philosophy of Mind”, which appeared on pp. 142-169 in Habermas 1973.

2 The text is easily available, e.g. Snow 1993, which contains the original lecture of 1959 plus The two cultures: a second look, written five years later. I use Kreuzer 1987. 
must have been puzzling to the young student who in the early morning was listening to a great electrical engineer, did his mathematics around noon, a bit of programming after lunch, and went to hear about Peircean semiotics in the late afternoon, just before rushing away to the opening of some artist's exhibition.

But now he had to swallow the fact that there was no communication, no interaction!? How could that be possible? Wasn't he himself doing exactly this: moving back and forth between those areas that - to be sure - had their own ways of expressing their findings, certainly, but wasn't communication happening through his own activities, even if restricted to himself and his friends? A decade later, in 1968, the great outburst of the youth's revolution against the father's generation shook the country. First readings about work and exploitation and suppression followed - a lot of Marx some years after this, and then, a strange sort of revelation.

Against the largely mathematical background of computer graphics, the open and heated discussions about human-computer communication were pure excitement. I had published a paper in 1984 on the impossibility that humans could ever communicate with computers, if the term "communication" was to be taken seriously. ${ }^{3}$ Unix was ruling, Silicon Graphics machines were great, but the Apple Macintosh had appeared on the market. We were beginning to interact with the computer as never before. The researchers at Xerox PARC had done tremendous things that made beautiful surprises in the classroom. C. P. Snow's verdict was still present in my thinking. But now came the discovery of that second word in the title of Habermas' essay: interaction!

Reading, on page 9 of this collection of supposedly critical texts by Habermas, the word Interaktion not only meant that you were, perhaps, no longer restricted to saying "HCI" when you were talking about humans and computers and their intricate relations. It meant that you could, perhaps, add a totally different perspective than the one usually offered in computing circles. So what did I learn, how did I read Habermas?

In a nutshell, Habermas, in my reading and restricted conclusion, said the following. The mediation of subject and object is what constitutes "Mind" (Geist). There are three ways how the subject and the object may be related. Three categories mediate between subject and object. The three categories are language, tool, and family. These terms stand for three patterns of dialectic relations, the patterns of symbolic representation, labour process, and reciprocal interaction. 
The tool and labour process stand for that mediation of subject and object, where the subject changes the state of the object. The tool-relation transforms the object into a state better fit for the subject's needs.

The language or symbolic representation stands for that mediation of subject and object, where the subject observes and describes the state of the object. The language relation creates a semiotic layer that stands for the object.

The family or reciprocal interaction stands for that mediation of subject and object, where the subject accepts the object as of the same kind and capacity as the subject itself. The interaction relation leads to cooperative, communicative exchange between subjects that are equal.

Mind you, this is the simple, naive, and immediate interpretation of a probably difficult reflection. The interpretation was by a computer scientist who was quite happy to find something in the other faculty which he thought could help him. The writing was by a philosopher and social scientist who, by the time of his writing, was developing his theory of communicative action, which played an important role in his attempt to reconstruct, as he said, dialectical materialism. Undoubtedly, dogmatists must have fiercely attacked him for working on such a project, whereas more liberal representatives of the left may have accepted the premise that Marxism also has the right to evolve when social reality changes. ${ }^{4}$ My naïve view, however, quite happily suggested to me that a systematic approach could be applied in order to introduce an interactive mode of using computers. I may have, of course, grossly misunderstood, nevertheless, the threefold distinction may serve a purpose here.

In the rest of this essay, I will describe three cases of using computers. Each case involves two persons in varying positions relative to the machine. In each case, we will see the open surface of the computer periphery, which will be commented on. We will also see the hidden and more or less inaccessible subface, and will gain some insight into how, and why, the two are necessarily related to each other. We will discover what the reader may already have been aware of all the time: computer things come in pairs. We will briefly give a semiotic interpretation of this claim by introducing the concept of an algorithmic sign. In conclusion, we will point to the current importance of digital media as those media that explore the dialectics of algorithmics and aesthetics.

$4 \quad$ Keane 1975 , pp. $82-100$ 


\section{Scene 1: Automaton. Two persons interacting, waiting for the computer}

The year is 1963 or 1964 . Two men are sitting in front of a computer. The size of the room may be 15 by 6 meters. No-one else is in there. They are not really sitting in front of the computer. The racks and cabinets that make up the computer create a system way too large to be sitting in front of. Besides, the two are sitting inside a separated space surrounded by glass walls. They see the metal cases, lined up in a long row of several meters length, and the tape drives next to the cases. Inside the glass room, temperatures are a bit more agreeable for humans. Outside, the climate must be closely monitored to stay within a small margin to prevent frequent failure of operation. The peripherals of the machine share the glass-walled space with the two men; a paper tape reader and a tape printer next to a large computer console.

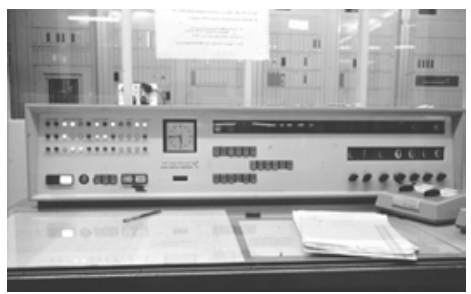

Fig. 1. The operator's interface. Computer SEL ER56 at Computing Centre, University of Stuttgart 1965. We see dials (lower right) and push buttons to get at specified data. Through the glass in the background some of the hardware

One of the men has recently graduated in mathematics. The other one is a researcher in mechanical engineering. His theoretical investigations have led him to describe the behaviour of a metal sheet as a non-linear fourth order differential equation with boundary conditions. The equation can only be solved numerically.

When the researcher approached the computing centre for help, the young mathematician was given the job of cooperating with the senior person. At their first meeting the engineer explained what he was doing, why he was interested in getting the solution, even an approximate one, and that he hoped the younger man would do all the programming since he himself had no clue of what might be involved and could see no chance of changing this. Why should he learn to program? He would rather agree on meetings and the admittedly arduous work of cooperation.

A great chance for the young mathematician. He did not take much interest in the details of the mechanics. For him, it was enough to accept the differential equation as his starting point. To which extent it described the vibrations of the sheet was the engineer's responsibility. As a mathematician, he was responsible for the selection of a modern numerical method for numerical integration (he chose a Fehlberg algorithm). Fine tuning for efficiency in those old days was an important and creative task. You could come 
up with improvements week after week. You did the improvements yourself, no compiler could take the burden from you (in fact, there was no compiler on this machine). But sooner or later the program worked and production could begin.

Since this was a boundary value problem, another difficulty had to be tackled: how to satisfy the boundary conditions? Such conditions require that a valid solution starts and ends at specified locations. It must leave and arrive there under specified directions (making a total of four conditions).

The rather simple approach was trial-and-error: Start from the prescribed position at the left end; solve an "initial-value" problem by arbitrarily assuming two more initial conditions; compare the calculated endpoint to the right with the prescribed goal. If there is a difference (which will, most likely, be the case), adjust the arbitrarily chosen initial condition such that, hopefully, the discrepancy is diminished, and repeat. This shoot-and-run approach will, under not too heavy circumstances and after some systematic attempts, come close enough to a realistic solution.

In those days, computers were incredibly slow when you compare them with their performance now, fourty years later. The difference in efficiency must be 6 to 10 degrees of magnitude, if not more. The two men, after having started the next shot, had to wait for several minutes (up to five), before the machine presented the few numbers they needed to judge how close the shot had come to the goal. While waiting, they had plenty of time to talk about mechanics, mathematics, programs, artificial intelligence, philosophy, politics, university gossip, the latest jazz concert, and a dozen more topics.

The situation thus indicated is heavily interactive! The two intellectuals were cooperating to solve a tough problem (they actually spent about two or three months before enough data had been generated from the virtual experiments in the algorithmic laboratory). Enough data was achieved when the engineer decided that his theoretical model was now backed up by enough empirical evidence. The cooperation between the two men allowed them to interact in the most complex and interesting ways. No procedures, no methods prevented them from journeying down any of the myriads of alleys open to the mind. The two actually became friends.

Embedded into the human-human interaction were short moments of very low-level human-computer interaction. When the result of the last calculation had become visible, the two discussed it and decided how to proceed. Proceeding was defined by a choice of new initial values. The process of choosing could have been automated since the goal was well-defined. But the amount of extra calculations would then, very likely, increase much more rapidly than with personal inspection. Inspection and discussion took advantage of the human capacity to detect patterns, consider context, and be aware of the situation and its changes. 
The machine they were using, an early transistorised decimal (!) computer was good enough for the purpose. Its interface displayed current numerical values of data stored in memory cells. The two friends, in order to read the coordinates of the final destination, had to dial knobs to get at those memory locations (given by their absolute addresses), and then lock up the numerical contents of that location.

At the machine level, only a tiny bit of interaction was happening. Really, this wasn't more than looking up and reading some signals - ridiculously lowlevel when compared to what became standard twenty years later. Slow times those days, you would say. Two men engaged in watching the computer come up with the result of a calculation. They had to wait because the machine was used as an automaton. They fed the automaton with data, hit the start button for the automaton to do its work, and were thrown back to talking, becoming bored, or listening to the radio.

\section{Scene 2: Tool. One person using the computer, the programmer far away}

The year is 2002, nearly forty years later. In terms of technology, the world has turned upside down. The technical infrastructure of all processes, in the private, economic, administrative, or political realm, is determined by data processing. In parts of the world, there is virtually no room that does not contain at least one computer. The art historian is sitting at her desk at home. Her current field of interest is an area not well known, certainly not mainstream, but slowly and steadily gaining interest. Already in her Ph.D. thesis a few years ago she started to seriously study the phenomenon that in the mid 1960s was called "computer art". She now generally prefers to use the term "digital art".

She is preparing for a meeting with her students. Recent work by Manfred Mohr will be the topic. He is the German artist who first got access to a computer controlled drawing machine (often called a "plotter") in 1969 in Paris. He gave up painting in favour of programming, stopped using colour in favour of black and white and, later, some grey and silver. He gradually became a recognised artist who could make a living from selling his art. He had discovered his topic in the early 1970s: the cube and its symmetries. In order to gain complexity, the cube had become the hypercube of four or five or even more dimensions. Like other artists before him - say, e.g., Paul Klee, Josef Albers, the concrete artists - he had become a researcher as well. 
The sensation happened in 2001 when he exhibited large canvasses in bright colours. ${ }^{5}$ Our art historian was trying to understand those pictures. They had again been exhibited under the title space.color in October 2001 at the Museum für Konkrete Kunst in Ingolstadt, Germany. Ever since, Mohr had been using coloured fields in his paintings (and, later, computer installations). He needed six- or eleven-dimensional space and colour to increase complexity by orders of magnitude.

The art historian at her home desk, not being acquainted with geometric spaces of higher dimensions, tried to understand the algorithmic process that generated some of the pictures in front of her. The algorithm behind the canvas had been Mohr's secret for all his productions. In his catalogues he had been friendly enough to publish brisk and sober definitions of the algorithmic behaviours of the generative processes. But now, with colour reappearing in Mohr's works, she felt lost. She had been studying the catalogue of the Ingolstadt exhibition: reading statements, analysing pictures. She was unable to grasp how the remark that something was going on in six dimensions could be helpful. She turned on her laptop computer. Someone had
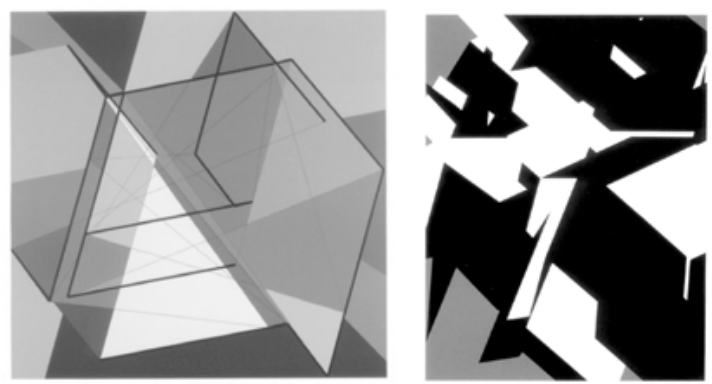

Fig. 2. Left: Manfred Mohr: P-107-f(1999). Right: P-1011-z2 (2004). Six dimensions behind the left, eleven dimensions behind the right image given her a program called deviceX. It was supposed to help develop some kind of understanding of the space.color period of Mohr's art.

The name, deviceX, rang two bells in her mind: tools and X-rays. Tools are instruments we use to more easily change the state of some material; X-rays are dangerous but helpful in looking into the human body. DeviceX could, perhaps, be a tool to look into the structure of those paintings.

Mohr's paintings of the space.color variety appear - just like any other painting does - as a configuration of coloured forms. The configuration of the forms corresponds to the geometry of the painting. We may derive from the geometry of a painting a more abstract rendition of the same content. This abstract rendition may be called the painting's topology. The abstraction gives

5 For the first time, Mohr showed these pictures in June 2001 at Galerie Wack in Kaiserslautern, Germany. 
up the particular form of an area, its size and, to a large extent, its location. Of the geometry, the topology keeps only one relation: that of neighborhood. If two areas are neighbors in the geometry, they must also be neighbors in the topology. So if they have a common edge in geometry, their topological neighbor-relation is an edge-neighborhood.

Topology is abstract. It gets described in formal symbolism. We can, however, use a minimal visualisation by using squares as the only form features. DeviceX makes use of this. The art historian started the program. Soon she found out how to use it. Among some other features not of prime interest here, the most prominent one is the following (Fig. 3). There is a small replica of one of the possible Mohr paintings. It can be grabbed with the mouse device and shifted horizontally, left and right. As it is moved further to the right, the intricate geometric forms untangle more and more into an arrangement of coloured squares.

We detect, as squares, the same colours as in the original picture. We also detect some that were hidden before. DeviceX is like a slider, i.e. an instrument we use to set one parameter to a certain value. The slider's relative position usually indicates the parameter's value along a scale from 0 to 1 .

DeviceX also functions according to this scheme, with one important difference, however: it does not indirectly indicate the parameter's value. It rather shows it directly. The device is loaded with the contents it controls. By looking at the slider we look into the picture. This is its X-property: the property of looking into (or even through) an invisible material.

The art historian, when applying deviceX to some data content of which she doesn't really know where it exists, considers herself a "user" of the software. The mode of use is usually called "interactive". The interaction is between her and the software, deviceX. It is quite clear to her that she is not shifting deviceX but the mouse in her hand. But it appears to her as if she was directly (and not indirectly) shifting the graphic rendition on her laptop's screen. The interaction between her and the computer has become so fast

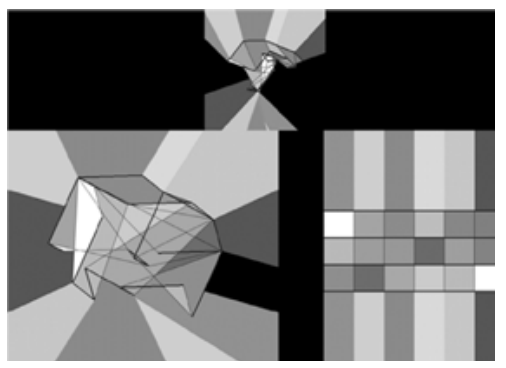

Fig. 3. deviceX. Top: Geometry of an image (lower left), topology (right) and intermediate state of slider (above). Bottom: a series of states of an image in transformation from geometry (left) to topology (right)

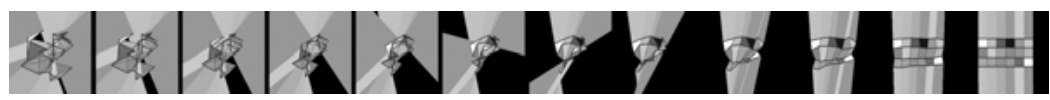



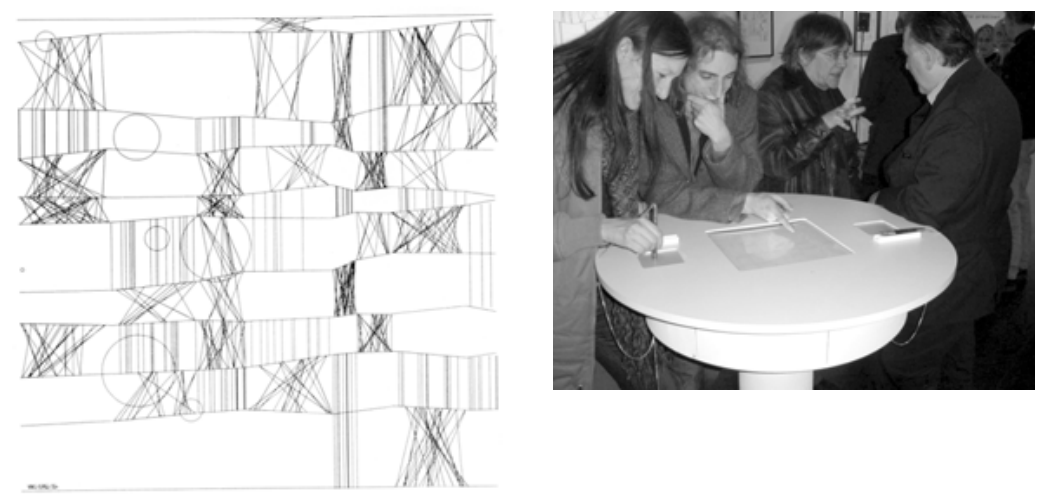

Fig. 4. Left: Frieder Nake, Homage à Paul Klee. Computer drawing, 1965. Right: Frieder Nake, Susanne Grabowski, Matthias Krauß: Spannung. Interactive installation. Picture taken during the opening of my exhibition at ZKM Karlsruhe: to the far right, Peter Weibel in discussion

that she can ignore the tremendous amount of calculations going on at every single moment.

The art historian probably also knows that the immediacy on the screen is caused by a programmer. He may now be living far away, working on something new. ${ }^{6}$ But once in the past, he wrote that program that now effectly appears like a tool in the art historian's hand. In some metaphorical way, it is a tool.

\section{Scene 3: Medium. Two persons having fun, others watching, the computer: where?}

It is now 2004. We enter the Korbakow room at Kunsthalle Bremen on our tour of Die präzisen Vergnügen 7 ("precise delights"). We observe a couple having fun at a bistro table (Fig. 4 right). A screen is mounted into the tabletop. Two small graphics input panels can be operated by using a pressure sensitive pen. The screen in the middle displays a line drawing belonging to the well known "Homage à Paul Klee" program (Fig. 4 left). There is a tremendous difference between the old algorithmic drawing of 1965 and the interactive installation of 2004 .

6 The programmer to be credited here is Matthias Krauß; see Nake/Krauß/Grabowski 2007, pp. 137-144.

7 The retrospective show of algorithmic works of Frieder Nake from the 1960s was put up under the condition that he could also present four new interactive installations. They were the result of collaborations with a group of students. 
The algorithmic drawing displays a complex structure of straight lines. Some run horizontally across the entire format. Others build bundles of parallel verticals. They are much shorter, and run from one of the horizontals to the next or next-but-one. There are also substructures of oblique lines building rhizome-like groups. A number of circles seem to be floating on or above the straight-line structure.

Each line and each structure of the static, algorithmic drawing is kept in its place by some mechanic force. All the hundreds of lines stick together keeping a graphical balance. This observation became the starting point for a dynamic and, in fact, interactive adaptation: each line was interpreted as a force, as a spring.

A system of springs is kept together by attachment points. These springs are not visible. They are a metaphor for an invisible property of each of the lines. The visible lines are a graphic interpretation of the spring system.

When a visitor puts the tip of one of the two available pens onto one of the graphics input panels, some line or point or cell is highlighted in colour. This feedback tells the visitor, which one of the objects he picked. Depending on the kind of objects, the visitor can perform a number of possible actions.
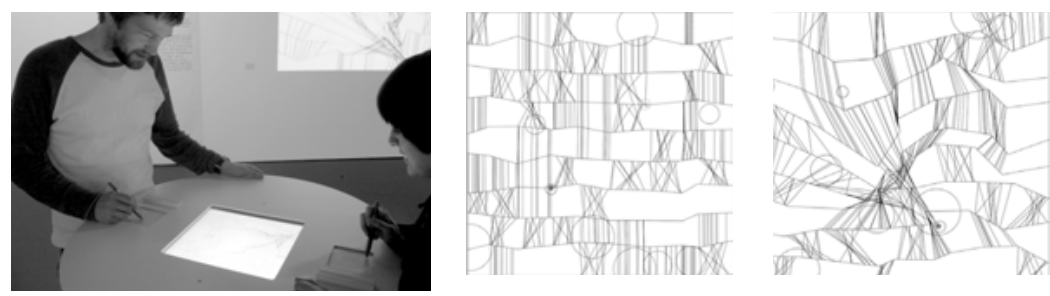

Fig. 5. The interactive installation Spannung. Left: Two visitors operating (display, two panels, pens; projection visible in the background) Centre: Display image with visitors' identified vertices (coloured dots). Right: Distorted image

He may move the contents of one of the horizontal bands of lines. By their spring property, they remain attached to the horizontals at all times (Fig. 5 for two visitors in action).

The visitor may also change the contents of a cell (from obliques to verticals to empty). Or he may grab one of the vertices, and pull it to a new location. This creates the most exciting effect because the entire structure must follow exactly, keeping its attachments as they were before.

The effect of applying an outside force to test the tensions inside the drawing becomes even more dramatic when two visitors grab vertices simultaneously and pull in different directions. This is an illustration of the basic 
characteristic of computing: objects are always double. We will describe this in different ways in the next section.

Here we should add how the field of forces influences the slow motion of the circles mentioned above. They have displayed a very calm and slow motion at all times even when the lines were static. The circles thus invited the visitor to think about the picture in dynamic terms.

The circles possess another unique property. Two visitors may grab them simultaneously. With one circle attached to the interactive tools of the visitors, they can move the circle around or change its size by pulling in different directions.

When no one is operating the installation, it displays a static minimal picture: a black square and a black circle, slightly overlapping, somewhat reminiscent of Malevich's suprematism. As soon as one of the pens is applied, one of the Homage à Paul Klee drawings slowly unfolds out of the square-andcircle. At the same time the image is projected onto the wall.

Pictures in a museum or gallery are silent witnesses of their artist's work, of their epoch, and of systematic and historic contexts. When we walk the halls of the museum, we may not get anything from the rich context. When we read a book, listen to a guide, or engage in conversation with friends, the situation changes, and the paintings also.

At first sight the interactive installation seems to be similar. But it is ready to tell us more about itself if we interact not only mentally, but manually as well. Interactive use of a program - today the ubiquitous mode of use - belongs to the characteristic view of the computer as medium. The exhibition in 2004 had installations that were to be used without tools. A camera detected passers-by and a minimalistic static projection was set into motion.

The message is: I am waiting here for you to do something; you don't have to be instructed, just do what you see fit, and I react. The interchange between you and me may tell you something, but what that could be, is totally up to you. My aesthetics is the unfinish. I am finished as far as I am a technical product. But I am obeying the law of unfinish ${ }^{8}$ (a funny kind of activity, isn't it?) by going on and on with no end, no goal.

This is exactly the identity of digital media. The computer has disappeared. Where is it, we may wonder? Hidden somewhere, having become a medium. Media are ubiquitous and unobtrusive.

8 The term is introduced in Lunenfeld 1999, see his introductory essay Unfinished business, p. 7. 


\section{Surface \& Subface or the Algorithmic Sign}

The three scenes described above stand for stages in interaction related to computing machinery. Its acknowledged history tells us that, without doubt, the computer started as an automaton. This does not only apply to the mathematical term automaton as used in formal theory. It applies much more to the historic evolution of the work of machines that had no choice, under given economic and political circumstances, but to give rise to automatic machinery. The concept of algorithm and the paradigm of computability stand for this.

The computability feature got wrapped into a tremendously beautiful and successful adorning layer of pseudo-tools. They are programs which no one must think of as programs. You start and stop them, specify their input parameters, and observe their outputs in the most joyful way. Never has machinery made use of its own capabilities so inventively, humbly, progressively, and aesthetically as computing technology. From the first bold steps by Alan Kay and his group at Xerox PARC in 1971 to Apple's first Macintosh and its software in 1984, only a dozen years passed. Ever since no other use had any chance, no other mode of existence had any relevance, but the tool perspective.

The economy of the time required the transformation of the computer into a market commodity. The concept for this was the invention of algorithmic tools; the mental paradigm was interactivity. As it evolved, and as miniaturisation continued at a breath-taking speed, algorithmic tools disappeared into the general environment. The third phase of interactivity brought back the original situation. In the first scene, we had seen two men interacting with each other - and, in the true sense of the word, there is no interaction other than between humans. The two, in their deliberations, occasionally turned to the computer to get answers to certain well-defined tasks.

The tool phase pushed groups of humans into the background in order to generate the false ideology of human-computer interaction. Human interaction had to be brought back artificially by inventing CSCW - computersupported cooperative work. But the tool phase was necessary and of the utmost importance. Without mercy, it caused everybody to use computers as an absolute given. Nobody can now work or live anymore without a computing machine.

The art world has widely accepted interactive applications of computability. It has provided another layer of wrapping paper: the transformation of the automaton into a medium. A great story of only forty or fifty years: The algorithmic revolution (Peter Weibel)! It depends on a very simple technical circumstance. I want to mention it at least briefly, giving it two names. Whatever 
thing you may address, choose, pick, apply, use on or in a computer, it comes as a sign of a special character. I call it the algorithmic sign.

The second name I want to attach to computer things is the pair of surface and subface. Let me explain this use of language before I take up the algorithmic sign, which is the more theoretical twist.

When we use a computer, we use a program running on the computer. A program - called software - is running on a computer, when a machine called hardware - is executing a code. The code is a sign for the program. When the program is running, it generates images on the computer display monitor, its main output device. Those images change in extremely rapid sequences. Each movement of the mouse or hit of a button changes the current image.

Screen images are visible to us. The program exists for us by its name and the world of images it generates. We anthropomorphise the operation of the program. We tell each other things like: "you should see what the new version of $\mathrm{X}$ is doing! It can now take your pictures and organise them such that it becomes much easier for you to ..." Thinking twice about such talk reveals a false conception. The program is really behaving just like any other machine: it is carrying out exactly what we want it to do, or at least, what our parameter settings force it to do.

A metaphorical way of talking about the program's behaviour is, nevertheless, justified. It is justified because the program is manipulating in its innermost organs what we only see as the current state of affairs. From an outside perspective, we may collapse this into one observation. Whatever is to become an utterance of the program for us to perceive, must first be stored in the display buffer to which the image on screen is tied in a one-to-one correspondence.

The screen is the surface, the display buffer is the subface of the algorithmic thing that the two of us - we ourselves and the program - are engaged in. The algorithmic thing comes as a visible appearance for us. At the same time, it comes as a computable appearance to the program. Without both being present and being tied to each other, nothing would work the way we want it to work. It does not make any sense to talk about the computer image without keeping in mind its visibility and computability, i.e. its computable visibility and its visible computability.

The computer thing is a double insofar as it is not only visible, nor is it only computable. It is visible in a new meaning of the word, and it is computable in a new meaning. Our thinking needs an understanding of the old meanings of those two concepts. Computer images are more than visualisations of a computation, and they are more than computations of an image.

The world of the surface and the subface that cannot but appear together is apt to catch exactly this: the inherent duplicity of anything happening on 
the semiotic machine. "Semiotic" is the correct word to characterise this. The algorithmic sign, which I am now going to introduce, is the theoretical concept for this semiotic perspective.

To recall from Charles S, Peirce, a "sign, or representamen, is something which stands to somebody for something in some respect or capacity. It addresses somebody, that is, creates in the mind of that person an equivalent sign, or perhaps a more developed sign. That sign which it creates I call the interpretant of the first sign. The sign stands for something, its object."

Of the many definitions of the sign by Peirce, this may be the most lucent one. To repeat in my own words, Peirce introduces the sign as a triadic relation, which wraps up the old dyad of something standing for some other. The sign, as a relation, cannot be perceived. But it must possess the feature of being perceivable. This feature is called the representamen. To be perceivable by our senses, it must be material. In some way, the representamen carries the sign. It carries it insofar as it gives rise to the relation that the perceiver is creating upon her perception. She is creating the object and the interpretant. The two together constitute what traditionally is called the meaning of the sign.

In Peirce's great analysis, the meaning of a sign depends on a culture, on a context, on a community. That community makes it possible by all of its conventions, history, habits, etc. for us to use the sign in the interests of communication. This general or public component of the meaning of the sign is the object of the sign.

Each subject perceiving a representamen and trying to make sense of it, also creates a particular or private component of the meaning of a sign: the interpretant. If the object is the long-lasting and generally accepted meaning of the sign, the interpretant is its short-term, situational and individually generated meaning.

Peirce thus gives us a dual way of talking about the sign: perceiving the red light of the traffic sign with the general and enforced interpretation of "Stop!" as well as the particular and deviating interpretation of "Proceed with great care!". The interpretant, by the way, is itself a sign. This introduces the sign as a recursive concept, as a process without end. Only the pragmatics of a given situation force us to interrupt the infinite sign process of interpretation.

Let us now take a look at what the computer does when it receives an input signal. The signal corresponds to the representamen of a possible sign. Of course we expect the computer to function well and to do exactly what the input signal "tells" it to do. With the rare exception of a malfunction, our expectation comes true. Nevertheless, the computer performs an act that 
formally is of an interpretive nature even if the computer is not capable of any interpretation. It is programmed in a definite, and precise way. The program "interprets" the input signal, i.e. it determines the one, and only one, interpretation of the line of code, or the command that it is then forced to execute.

The formal act of interpreting a unit of code, in the case of the program, reduces to a determination. Determination is the limit case of interpretation: finding out the one and only meaning. Thus algorithmic signs are signs in the usual (Peircean) sense of the word, but with an extra interpretant. We call this the causal interpretant to distinguish it from the intentional interpretant. The latter one is what the human creates. ${ }^{10}$

We now close the ring. The surface of any object on the computer corresponds to the intentional interpretant of the computer sign. The subface corresponds to the causal interpretant. I am not saying that the subface is the causal interpretant. For my intention here is to point at a correspondence between two perspectives.

The components of digital media, of semiotic entities on a computer, or of things we are interested in when using a computer, can only be understood in the world of relations, not in the world of things. This is my message in this essay. What is usually called the interface between human and machine, appears as the coupling of surface and subface. Both are machine-bound. Both are faces at which one process ends, and another process starts. The human places rather trivial components onto the surface (like mouse positions, or menu selections). He interprets what the program delivers in a rich way, influenced by his intentions, interests, situation, and context. Once the surface is transformed into the subface, the program starts its signal processes, which consist of chains of determinations like any other process on a machine.

The miracle of human-computer interaction is that it impossible as interaction in a true sense of the word. It is happening nevertheless. This is possible because human acts of interpretation correspond in a rich (but computable) way to machine operations of determination. The miracle is that humans were bold and intelligent enough to establish this. The miracle is not that machines were so intelligent to do it.

10 This concept is further elaborated in a chapter of the book by Peter Bøgh Andersen and the author (forthcoming). 


\section{Algorithmics \& Aesthetics}

A very brief remark in conclusion. Since about the mid 1990s, some aspects of computing science have been collected under new programmes of study. They are often called Digital Media, or something similar. These programmes carry the heavy burden of striking a balance between a serious and appropriate study of algorithmics up to the point of script programming, and of aesthetics of 20th century art. They need a bit of art history as well as the history of computing. Their questions should be directed towards an understanding of the algorithmic sign in as many ways as possible - aesthetic, educational, and cultural. 


\section{References}

Andersen, Peter Bøgh/Nake, Frieder (in preparation): Computers and Signs. Prolegomena to a Semiotic Foundation of Computing Science, Heidelberg: Synchron Wissenschaftsverlag der Autoren.

Habermas, Jürgen (1969): »Arbeit und Interaktion. Bemerkungen zu Hegels Jenenser >Philosophie des Geistes«. In: Jürgen Habermas, Technik und Wissenschaft als »Ideologie«, Frankfurt a.M.: Suhrkamp, 9-46.

Keane, John (1975): »On tools and language: Habermas on work and interaction«. New German Critique 6, 82-100.

Kreuzer, Helmut (Ed.) (1987): Die zwei Kulturen. C. P. Snows These in der Diskussion, Stuttgart: Klett-Cotta.

Lunenfeld, Peter (1999): The digital dialectic. New essays on new media, Cambridge: MIT Press.

Nake, Frieder (1984): »Schnittstelle Mensch - Computer«. Kursbuch 75, 109-118.

Nake, Frieder/Krauß, Matthias/Grabowski, Susanne (2007): »The sound of silence in spaces of many dimensions«. In: Biennale of Electronic Arts Perth: CADE: Computers in Art and Design Education Conference, 12-14 September 2007. Conference Proceedings, 137-144.

Peirce, Charles Sanders (1955): Philosophical Writings of Peirce. Selected and Edited with an Introduction by Justus Buchler, New York: Dover Publications.

Snow, Charles Percy (1993): The Two Cultures, Cambridge: Cambridge University Press. 


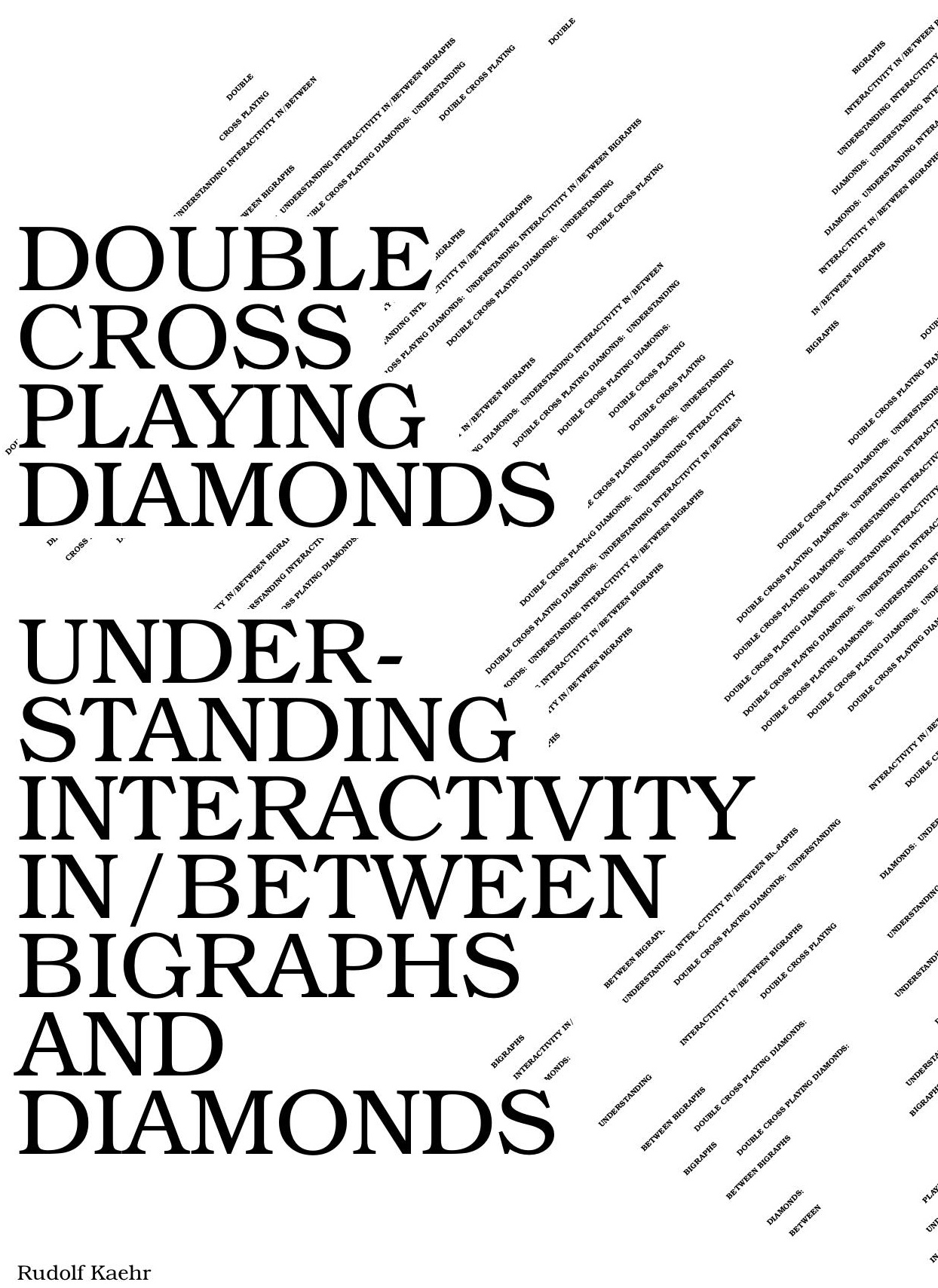



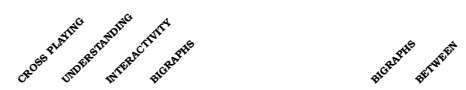

\section{Models of Interactivity between flows and salti ${ }^{1}$}

"Interactivity is all there is to write about: It is the Paradox and the Horizon of Realization."

Grammatologically, the Western notational system is not offering space in itself to place sameness and otherness necessary to realise interaction/ality. Alphabetism is not prepared to challenge the dynamics of interaction directly. The Chinese writing system in its scriptural structuration is able to place complex differences into itself, necessary for the development and design of formal systems and programming languages of interaction. The challenge of interactionality to Western thinking, modeling and design interactivity has to be confronted with the decline of the scientific power of alphanumeric notational systems as media of living in a complex world. ${ }^{2}$

The challenge I see for media artists is not only to develop interactional media constellations but also to intervene between the structures and dynamics of interactional systems as international corporations, governments, military and academia force them on us. ${ }^{3}$

\subsection{Comparison of two approaches to interactivity}

This paper takes the risk to compare two fundamentally different approaches to interaction and reflection in computational systems: Milner's bigraphs and diamond theory. Milner's bigraph model and theory of interaction is highly developed, while the diamond model applied to this interactional scenario and confronted with the bigraphs model is presented here for the first time.

The Milner model is presupposing a world-view (ontology, epistemology) of homogeneity and openness. Its basic operation is composition in the sense of category theory. Composition is associative and open for infinite iterability. Milner's model is a model of interaction in a global sense but it is not thematising formally the chiastic interplay of local and global aspects of interaction. Its merit is to have developed a strict separation of topography (locality) and connectivity for a unifying theory of global and mobile interaction (ubiquitous computing) surpassing, in principle, the limits of Turing computability.

1 Thanks to Marianne Dickson, Edinburgh, for bridging the corrections and correcting the bridges of this composition.

2 Kaehr $2006 a$

3 Kaehr 2003a, b
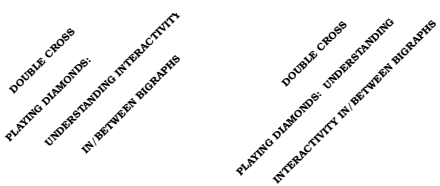
In contrast, the diamond model, which is just emerging, ${ }^{4}$ is based on an antidromic and parallactic structure of combination of events in an open/ closed world of a multitude of discontextural universes. In such a pluri-versal world model, each composition is having its complementary combination. With that, iterability for diamonds is not an abstract iterativity but interwoven in the concrete situations to be thematised, and determined by iterative and accretive repetitions, involving their complementary counterparts, without a privileged conceptual initial/final object.

This leads to a theory of diamonds as a complementary interplay of categories and saltatories (jumpoids) with the main rules, globally, of complementarity and locally, of bridging. Diamonds are involving bi-objects belonging at once to categories and to saltatories, ruled by composition and saltisition (jump-operation). ${ }^{5}$

\subsection{Interactionality as interplays between categories and saltatories}

In less technical terms, the polycontextural approach of diamond theory is supporting three new features:

First, it supports the idea of irreducible multi-medial contextures and their qualitative incomparability. That is, different media like sound, video, picture, text, graphics, etc., are conceived as logically different and as organised and distributed conceptually in a heterarchical sense. To thematise media as a digital contexture is not more than to emphasise their informatical and physical aspect, which is as such a contexture, too.

Second, it supports the possibility of mapping the (outer) environment of a contexture (media) in itself, i.e., to offer an inner environment for reflectionality. Contextures, to be different from systems, have to reflect their environment into their own domain. Hence, a contexture has to be understood as being involved into interplays of inner and outer environments.

Third, it supports the possibility of simultaneously realising movements (actions) and complementary counter-movements on a basic level of conceptualisation and formalisation. If composition of events inside a contexture, and mediation of different contextures to a compound contexture, polycontexturality, are characterised by the rules of combination, i.e., identity, commutativity and associativity, a new feature of composition is discovered by the diamond approach, which is antidromic and parallax, corresponding structurally to the otherness of the categorical system.

\footnotetext{
4 Kaehr 1996

5 Kaehr 2007a
} 
Therefore, the questions of interactionality in a diamond framework are not primarily, how do we globally move, physically and informatically, from one topographic place to another, but how do we move by interaction from one medium to another medium of a complex knowledge space. With the appearance of the semantic web and knowledge grid $^{6}$ such developments are unavoidable. Obviously, the polycontextural diamond approach is not opting for a principally homogeneous global field of informatical and physical events but for a discontexturality of different media, situations, contexts of meaning.

The Milner Model is well based, principally, on category theory, the diamond model has to develop its own new formalism, risked here as a diamondisation of category theory. Hence, both theories are in a constellation, which offers a reasonable possibility for comparisons.

Because the bigraph model is based on category theory and its concept of composition with its abstract iterability, the diamond model also has to develop a distinct concept of composition (combination), one which involves a complementarity of at least two different concepts of composition, i.e. the categorical and the saltatorical, and which is opening up the operativity of an open/closed concept of iter/alterability.

Even if only metaphorically and still vague, what is common to both models is their dichotomous, dual, complementary and orthogonal approach to interaction and interactionality. The Milner model is focused on message passing, flow of informatic objects, the diamond model on agents and their reflectional/interactional activities with an emphasis on intervention.

\section{Milner's bigraph model of interaction}

Out of his cloud of keywords to ubiquitous computing and interactivity, Milner chooses at his Beijing 2005 performance 3 leading features: locality, mobility and connectivity. ${ }^{7}$

\subsection{Locality and connectivity}

\section{Locality}

"Programming the digital computer ramifies the use of space and spatial metaphor, both for writing programs and for explaining why they work. This shows up in our vocabulary: flow chart, location, send and fetch, pointer,

\footnotetext{
6 Kaehr 2004b

7 Milner 2005, p. 49
} 
nesting, tree, etc. Concurrent computing expands the vocabulary further: distributed system, remote procedure call, network, routing, etc.

We are living with a striking phenomenon: the metaphorical space of algorithms - graph, array, and so on - is mixed with the space of physical reality."

\section{Physical and virtual space}

"Informatic objects flow in physical space; physical objects such as mobile telephones manipulate their informatic space."
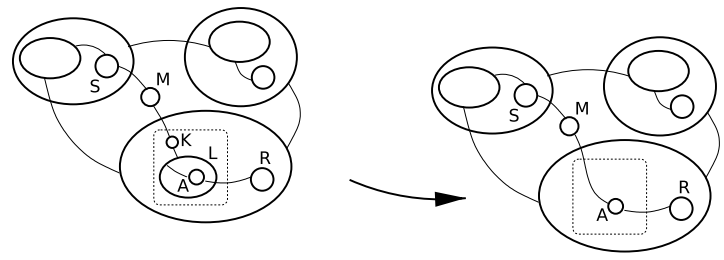

"The picture illustrates how physical and virtual space are mixed. It represents how a message $M$ might move one step closer to its destination. The three largest nodes may represent countries, or buildings, or software agents. In each case the sender $\mathrm{S}$ of the message is in one, and the receiver $\mathrm{R}$ in another. The message is en route; the link from $\mathrm{M}$ back to $\mathrm{S}$ indicates that the messages carries the sender's address. $\mathrm{M}$ handles a key $\mathrm{K}$ that unlocks a lock $\mathrm{L}$, reaching an agent $\mathrm{A}$ that will forward the message to $\mathrm{R}$; this unlocking is represented by a reaction rule that will reconfigure the pattern in the dashed box as shown, whenever and wherever this patterns arises."9

"Bigraphical reactive systems are a model of information flow in which both locality and connectivity are prominent. In the graphical presentation these are seen directly; in the mathematical presentation they are the subject of a theory that uses a modest amount of algebra and category theory. A bigraph may reconfigure both its locality and its connectivity. The example pictured above shows how reconfiguration is defined by reaction rules; in that case, the rule may be pictured thus:

The mathematical structure of bigraphs allows concepts to be treated somewhat independently; for example, connectivity and locality are treated orthogonally." 10

8 Milner 2007, p. 1

9 Milner 2007, p. 1

10 Milner 2007, p. 2 


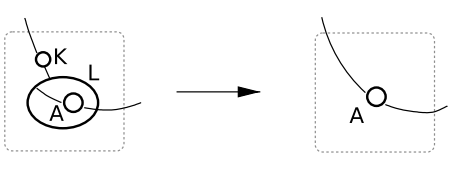

"So the challenge to bigraphs is to provide a uniform behavioural theory, allowing many process calculi to be expressed in the same frame while preserving their treatment of behaviour." 11

\section{The aim of a new design}

"The challenge for global ubiquitous computing is to devise theories and design principles in close collaboration, ..."12

"The long-term aim of this work is to provide a model of computation on a global scale, as represented by the Internet and the World Wide Web. The aim is not just to build a mathematical model in which we can analyse systems that already exist. Beyond that, we seek a theory to guide the specification, design and programming of these systems, to guide future adaptations of them, and not to deteriorate when these adaptations are implemented. [...]

This will only be achieved if we can reverse the typical order of events, in which design and implementation come first, modelling later (or never). For example, a programming language is rarely based thoroughly upon a theoretical model. This has inevitably meant that our initial understanding of designed systems is brittle, and deteriorates seriously as they are adapted.

We believe that the only acceptable solution, in the long run, is for system designs to be expressed with the concepts and notations of a theory rich enough to admit all that the designers wish." ${ }^{13}$

\subsection{Strategies of orthogonal simultaneity}

"So our strategy here is to tackle just two aspects - mobile connectivity and mobile locality - simultaneously. In fact this combination contains a novel challenge: to what extent in a model should connectivity and locality be interdependent? In plain words, does where you are affect whom you can talk to? To a user of the Internet there is total independence, and we want to model the Internet at a high level, in the way its connectivity appears to users. But to the engineer these remote communications are not atomic, but

11 Milner 2007, p. 2

12 Milner 2005, p. 64

13 Milner 2004b, p. 7 
represented by chains of interactions between neighbours, and we should also provide a low-level model, which rejects this reality. So we want to have it both ways; furthermore, we want to be able to describe rigorously how the high-level model is realised by the low-level one." ${ }^{14}$

\section{Milner's Model of bigraphs ${ }^{15}$}

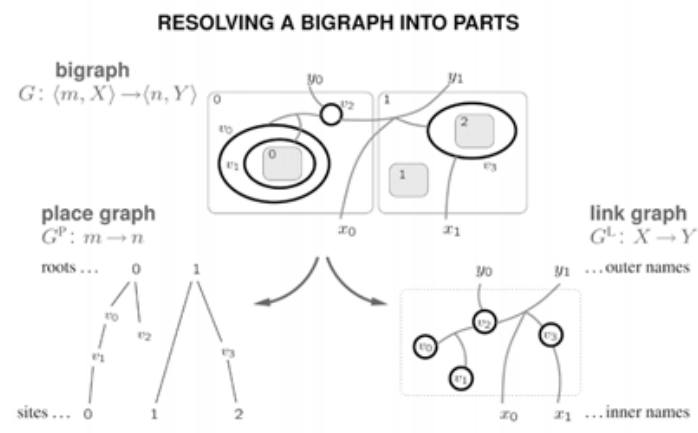

21

\subsubsection{Statics of interaction: Categorical framework}

"Abstract. This paper axiomatises the structure of bigraphs, and proves that the resulting theory is complete. Bigraphs are graphs with double structure, representing locality and connectivity. They have been shown to represent dynamic theories for the pi-calculus, mobile ambients and Petri nets, in a way that is faithful to each of those models of discrete behaviour. While the main purpose of bigraphs is to understand mobile systems, a prerequisite for this understanding is a well-behaved theory of the structure of states in such systems. The algebra of bigraph structure is surprisingly simple, as the paper demonstrates; this is because bigraphs treat locality and connectivity orthogonally." 16

\subsubsection{Dynamics of interaction: Labeled process calculi}

"Let us repeat: in a pure bigraph $\mathrm{G}:<\mathrm{m}, \mathrm{X}>-><\mathrm{n}, \mathrm{Y}>$ we admit no association between its outer names $\mathrm{Y}$ and the roots (regions) $\mathrm{n}$, nor between the

14 Milner 2004b, p. 7

15 Milner 2006, p. 21

16 Milner 2004a, p. 1 
inner names $\mathrm{X}$ and the sites $\mathrm{m}$. It is this dissociation that enables us to treat locality and connectivity independently, yielding a tractable theory." 17

The dynamics of bigraphs is formalised by labeled process calculi:

"The challenge from process calculi is to provide a uniform behavioural theory, so that many process calculi can be expressed in the same frame without seriously affecting their treatment of behaviour. We now outline how research leading up to the bigraphical model has addressed this challenge.

It is common to present the dynamics of processes by means of reactions (also known as rewriting rules) of the form $r \rightarrow r$, meaning that $r$ can change its state to $r$ ' in suitable contexts. In process calculi this treatment is typically refined into labelled transitions of the form $a \rightarrow^{1} a^{\prime}$, where the label 1 is drawn from some vocabulary expressing the possible interactions between an agent $a$ and its environment. These transitions have the great advantage that they support the definition of behavioural preorders and equivalences, such as traces, failures and bisimilarity. But the definition of those transitions tends to be tailored for each calculus." 18

\subsubsection{Formalisation of interaction: Bigraphs as tensor categories}

"This chapter establishes place graphs, link graphs and bigraphs as arrows in certain kinds of category. Any kind of category is concerned with operations upon arrows, especially composition." 19

"Note that this combination is quite distinct from the categorical composition used to insert one bigraph into another (e.g. an agent into a context). But it is simply related to them; to compose two bigraphs categorically, we first resolve them into their respective place graphs and link graphs, then compose these, and finally combine the results into a new bigraph." 20

\subsubsection{Axiomatics of bigraphs}

"The topic of this paper is to axiomatise the resulting structure of bigraphs. The justication for such a specific topic is threefold.

First, the work already cited gives ample evidence that a graphical structure combining topography with connectivity has wide application in com-

17 Milner 2004b, p. 20

18 Milner 2005, p. 8

19 Milner 2007, p. 13

20 Milner 2004b, p. 19 
puter science; for as we have seen it brings unity to at least three models of discrete dynamics, each of which has already many applications.

Second, it appears that the algebraic treatment of such dual structures has not been previously addressed; yet the behaviour of systems whose connectivity and topography are both reconfigurable may be so complex that their dynamics cannot be properly understood without a complete and rigorous treatment of their statics. Bigraphs are just one possible treatment of such dual structure, but it is likely that their static theory can be modified for other treatments.

Third, as we shall see, dual structures seem to require a novel kind of normal form which is essential to a proof of axiomatic completeness." ${ }^{11}$

\section{Axiomatics (Table 1)}

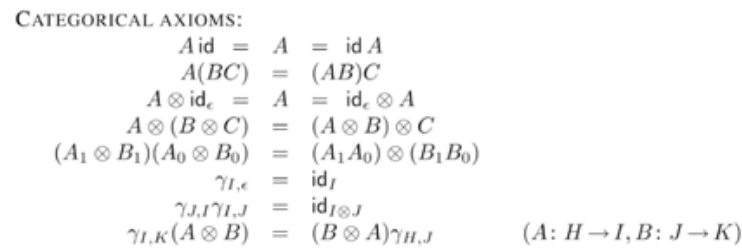

LINK AXIOMS:

$$
\begin{aligned}
/ y \circ y / x & =/ x \\
/ y \circ y & =\mathrm{id}_{\epsilon} \\
z /(Y \uplus y) \circ\left(\operatorname{id}_{Y} \otimes y / X\right) & =z /(Y \uplus X)
\end{aligned}
$$

NODE AXIOMS:

$$
\left(\mathrm{id}_{1} \otimes \alpha\right) \mathrm{K}_{\vec{x}}=\mathrm{K}_{\alpha(\vec{x})} .
$$

"In other words, the axioms are both sound and complete. They say simple things: The place axioms say that join is commutative, has a unit and is associative; the link axioms say that the formation of links obeys obvious rules; the node axiom says that we can name ports arbitrarily." 22

\subsubsection{Completeness of the axiom system}

"The completeness of the axiom system in Table 1 depends primarily on two things: first, that all linking can be exposed at the outermost level of an

\footnotetext{
21 Milner 2004a, p. 4

22 Milner 2004a, p. 23
} 
expression; second, that we have a strict symmetric monoidal category of bigraphs, with a tensor that is partial on objects. Crucial to the tensor is that it is bifunctorial, i.e. $\left(A_{1} \times B_{1}\right)\left(A_{0} \times B_{0}\right)=\left(A_{1} A_{0}\right) \times\left(B_{1} B_{0}\right)$; this axiom underlies most of our manipulations. Thus the discrete normal form, DNF, has been crucial for the proof of completeness." 23

\subsection{Orthogonality of topography and connectivity}

\subsubsection{Underlying world model}

The bigraph model of interaction is highly flexible and is liberating further research from unnecessary fixations. Bigraphical reactive (re-writing) systems as models of information flow are dealing with locality and connectivity as orthogonal events, distributed over two dimensions. Such a separation of structural locality and behavioural connectivity enables a clear modeling and an effective formalisation as a bigraph or bipartide system. Spaciality is conceived as static, formalised by category theory and behaviour as dynamic, formalised by process calculi (pi-calculus).

The bigraph model of interaction seems to belong to a world model with the characteristics of: "Everything in this world is changing but the world in which everything is changing doesn't change." ${ }^{4}$ Ubiquitous and global computing is presupposing an epistemologically uniform, homogeneous and unique world of physical and informatical events.

Diamond theory can be set in some kind of a correspondence with a bipartide model but it is turning to a world model where there are many worlds in which things are changing and in which worlds themselves are changing too. Diamond Theory is involved not in a new super-stable world but in the game of interactionality/reflectionality between worlds and events, hence enabling system designers and media artists to intervene in and between those worlds guided by the metamorphic dynamics of polycontextural diamonds.

Messages in the diamond model are conceived as polycontextural and as belonging simultaneously to different contextures of irreducible kinds of meaning. Message passing in such a model is not done by the metaphor of key/lock/unlock/agent in a location/connectivity setting because a key in this pluriversal world-model appears always as necessarily polysemic and its acceptance has to be negotiated by reflectional and interactional activities. If such complex transactions are becoming stable in their usage, a reduction to the mono-contextural key-model can be introduced by reducing complexity.

23 Milner 2004a, p. 21

24 Kaehr 2007d 


\subsubsection{Chiastic transition metaphor}

Hence, in a chiastic metaphor, we can state that statics in the bigraph model becomes dynamics in the diamond model; and dynamics becomes statics in the diamond setting because its dynamics is bracketed and moved into a multitude of process-structures wherein the dynamics of the different behavioural systems have an arena in which to act. Therefore, category theory as formalism for interaction has to be dynamised towards diamond theory. That is, category theory has to be diamondised towards a dynamic structural formalism, which is an operational structuration.

\subsubsection{Opting for an interventional design}

The British Grand Challenge project for computing is not touching the principle hierarchy between mathematics and informatics. Since the Greeks time has changed and a reversion and displacement of this hierarchy might be the grand challenge of a new understanding of global computing. ${ }^{25}$

From a model of interactions to a design of interactionality, the transitions to be risked might be:

From the global, ubiquitous and universal web of computation, to the kenomic grid of pluriversal contexturality, containing the chiasm of global/ local scenarios.

From the locality in the Actor model of informatical events to the positionality of contextures in the kenomic grid, positioning informatic localities.

From the mobility in the Actor model of informatical flows between ambients (context, locality) of the same contextural (ontological, logical, semiotic) structure to a metamorphosis between contextures, augmenting complexity/ complication of contextural scenarios implementing clusters of informatical ambients and mobility.

From the operations between actional ambients to the operationality in polycontextural situations realised by the super-operators (identity, replication, permutation, reduction, bifurcation) placing ambient operations into the grid.

From the connectivity of actions at a locality of message passing, using a key to unlock a lock of an agent, to different kinds of mediation between contextures containing informatical connectivity. 
These transitions seem to record a catalogue of minimal conditions to be fulfilled to realise interactionality/reflectionality and interventionality in such complex constellations as the emerging knowledge grid. ${ }^{26}$

\section{Diamond theory of interactionality}

\subsection{Diamond Strategy}

\section{Encounter}

Diamond strategies are sketching transitions from the mail model of interaction in bigraphs to the encounter model of interactionality/reflectionality and intervention.

Before we can play the bipartide game of locking and unlocking (by passing a key in a structure of orthogonal locality and connectivity to reach an agent capable of passing the message to another agent), the otherness of the actors involved has to be acknowledged and accepted by all the interactional activities of the actors involved.

It can be described as the action of addressing an addressee, which is able to accept the addressing by offering its own addressable structure. After having been addressed and having the addressing accepted by the addressed and after the addresser has recognised the acceptance of being addressed and the addressing is thus established, information can be exchanged between agents in the sense of communication. ${ }^{27}$

Interactivity in the encounter-model, therefore, is conceived as a mutual action of acceptance and rejection between different agents. Only on the basis of this interactional agreement can information exchange happen. ${ }^{28}$

Therefore, the structure of interaction is always complex: at once realising the addresser and the inner environment of the addressee. This simultaneity of inner and outer environments of agents involves a kind of structural bifurcation and mutual actions of acceptance and/or rejection of the involved agents based on the complexity of their architectonics. That is, the addressee has to give space (einräumen) to the addresser to be addressed. To address and to accept to be addressed is a mutual action of at least two agents in a common co-created environment. Hence, the actional structure of interactionality is not only bipartide but antidromic, too. This phenomenon forces a

26 Kaehr 2006b

27 Kaehr 2004a

28 Kaehr 2004a 
formalisation paradigm beyond mathematical category theory, which finds a very first attempt to a realisation in the proposed diamond theory. ${ }^{29}$

\title{
Intervention
}

An interaction of an agent, including reflections on the behaviour of a partner agent, which is intended to change the meta-rules of the partneragent can be called an intervention. An agent is intervening into an interaction in attempting to change the meta-rules of the agent. An intervention takes place if an agent is interacting with another agent in a way that the agent is forced to change his meta-rules to stay in the game of computation and interaction. ${ }^{30}$

\begin{abstract}
The aim is not just to build a mathematical model in which we can analyse systems that already exist. Beyond that, we seek a theory to guide the specification, design and programming of these systems, to guide future adaptations of them, and not to deteriorate when these adaptations are implemented. There is much talk of the vanishing ubiquitous computer of the future, which will obtrude less and less visibly in our lives, but will pervade them more and more. Technology will enable us to create this. To speak crudely, we must make sure that we understand it before it vanishes. ${ }^{31}$
\end{abstract}

Diamond strategies are not only asking for an understanding of such trends, like the vanishing of computational challenges for users by ubiquitous computing, but for the possibility of intervention by computer designers, scientists and users into such trends. Thus, opening up interplays between users and general computation, avoiding any kind of regression into euphoria, criticism and luddism of humanistic self-defence.

\subsection{Towards Diamond Theory}

\subsubsection{From categorical composition of morphisms to diamonds}

Actions from A to B can be considered as morphisms, symbolised by an arrow from $\mathrm{A}$ to $\mathrm{B}, \mathrm{A} \rightarrow \mathrm{B}$. In this sense, morphisms are universal, they occur everywhere. But morphisms don't occur in isolation, they are composed together in interesting complexions. The composition of morphisms (arrows) is defined by the coincidence of codomain (cod) and domain (dom) of the morphisms to be composed, called the matching conditions (MC). That is, (f, g)

29 Kaehr 2007c

30 Kaehr 2005, 2006c

31 Milner 2004b, p. 7 
is composed ( $\mathrm{f} \circ \mathrm{g}$ ) iff $\operatorname{cod}(\mathrm{f})=\operatorname{dom}(\mathrm{g})$. This highly general notion of morphism and composition of morphisms is studied in Category Theory. ${ }^{32}$

A general descriptive explication of the concept of composition of morphisms is given by the following diagram. It contains the table of the matching conditions. Here, the distinction between objects, A, B as domain and codomain properties of morphisms, and the alpha $(\alpha)$ and omega $(\omega)$ functionality of morphisms are included.
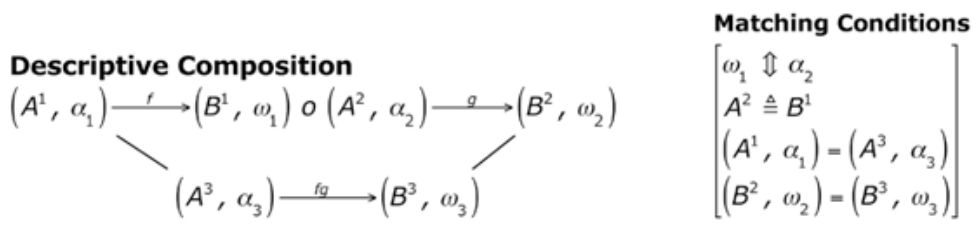

Hence, not only the codomain $\mathrm{B}^{1}$ and the domain $\mathrm{A}^{2}$ as objects have to coincide, but also the actional domain "alpha ${ }_{2}$ " $\left(\alpha_{2}\right)$ and the actional codomain "omega ${ }_{1}$ " $\left(\omega_{1}\right)$ as functional properties of the morphisms $f$ and $g$, have to match. Obviously, the commutativity of the diagram has to fulfill, additionally, the matching conditions for $\left(\mathrm{A}^{1}, \alpha_{1}\right)$ with $\left(\mathrm{A}^{3}, \alpha_{3}\right)$ and $\left(\mathrm{B}^{2}, \omega_{2}\right)$ with $\left(\mathrm{B}^{3}, \omega_{3}\right)$, defining the composition ( $\mathrm{o}$ g).

First, without the actional alpha/omega-notation we get the matching conditions, coincidences, for categorical composition based on the objectional distinction of domains and codomains.

Second, stripped off of the set-theoretical or objectional content of the domains and codomains of morphism, the functionality of beginnings $(\alpha)$ and endings $(\omega)$ remain. Composition then means an exchange relation between the ending of a morphism and the beginning of another morphism, i.e., between $\left(\omega_{1}\right)$ and $\left(\alpha_{2}\right)$. Both founded in the coincidence relation between the actional domain of the first and the actional codomain of the second morphism, establishing the commutativity of "object-free" categorical composition, i.e., the morphism between $\left(\alpha_{3}\right)$ and $\left(\omega_{3}\right)$, i.e., $\left(\alpha_{3}\right) \rightarrow\left(\omega_{3}\right)$.

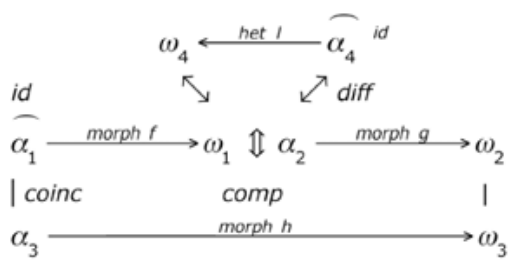

32 Kaehr 2007a 
Such a chiastic approach, emphasising the pure functionality of composition uncovers the possibility of a new relationship involved in the definition of actional composition: the complementarity of the commutative morphism between the beginning $(\alpha 2)$ and the ending $(\omega 1)$ involved in the categorical composition, building the "antidromic and parallax" hetero-morphism between $(\alpha 4)$ and $(\omega 4)$, i.e., $(\alpha 4) \rightarrow(\omega 4)$.

Hence, functional composition of morphisms, which are represented by order relations, is based on the functional matching conditions, $\mathrm{MC}$, of two types of relations: exchange and coincidence relation building together with the order relations, a chiastic pattern in form of a diamond. Obviously, this singular diamond is occupying a place and is localised in a grid of diamonds and thus ready to be disseminated.

Third, both thematisations together, the objectional and the actional, with morphisms and hetero-morphisms, define the diamond composition of morphisms.

\subsubsection{Diamond model of system/environment}

Some wordings to the diamond system/environment relationship might be listed:

What's my environment is your system.

What's your environment is my system.

What's both at once, my-system and your-system, is our-system.

What's both at once, my-environment and your-environment, is ourenvironment.

What are our-environments and our-systems is the environment of otherssystem.

What's our-system is the environment of others-system.

What's neither my-system nor your-system is others-system.

What's neither my-environment nor your-environment is othersenvironment.

$$
\text { Diamond-Obj }^{(4)}: \text { Obj }^{(4)} \rightarrow \text { Obj }^{(4)}
$$

system my envm

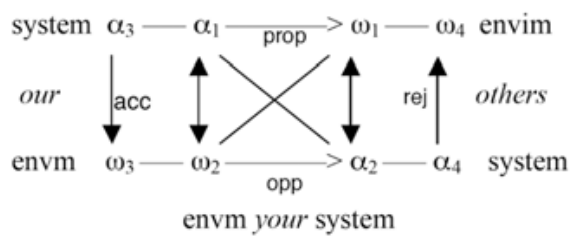




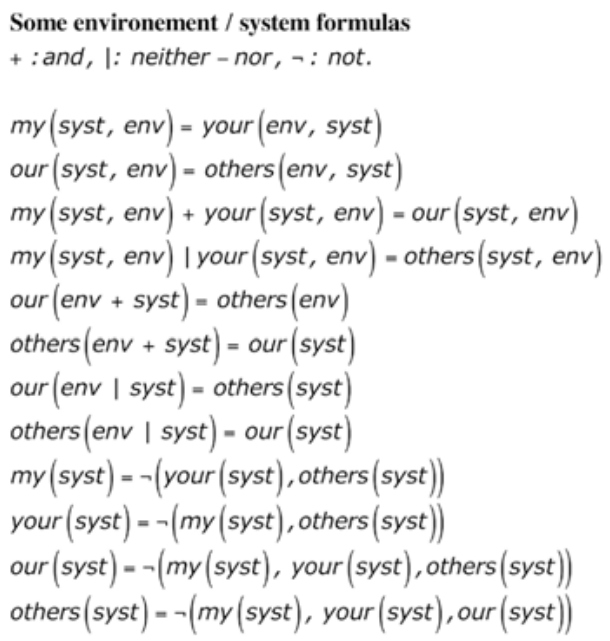

The diamond modeling of the otherness of the others incorporates the otherness into its own system. An external modeling of the others would have to put them into a different additional contexture. With that, the otherness would be secondary to the system/environment complexion under consideration. The diamond modeling is accepting the otherness of others as a "firstclass object", and as belonging genuinely to the complexion as such.

In another setting, without the "anthropomorphic" metaphors, we are distinguishing between a system and its internal and its external environment. The external environment corresponds to the rejectional part, the internal to the acceptional part of the diamond. Applied to the diamond scheme of diamondised morphisms we are directly getting the diamond system scheme out of the diamond-object model.

Much work has been done on interactionality/reflectionality and interventionality/interlocutionality on the basis of polycontextural notions and formalisms. ${ }^{33}$ Despite its chiastic and proemial approach, this work did not yet include the others-system of the diamond model.

33 Kaehr 2005, 2006d 


\subsection{Diamond Structuration}

Diamonds in this sketch are conceived as interplays between categories and saltatories based on morphisms and hetero-morphisms with their compositions, saltisitions and bridgings. Saltatories are the complementary concept of categories.

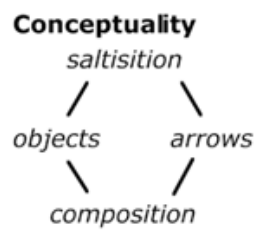

The conceptuality of diamond theory is introduced by an application of the diamond strategies to the basic concepts of category theory: objects and morphisms (arrows). Objects are understood in this setting as propositions, arrows as oppositions. Compositions appear as the both-at-once of objects and arrows, and saltisitions as the neither-nor of objects and arrows. Composition and saltisitions, hence, are complementary concepts.

\section{saltisition, saltatory}

salto mortale: jump from the apriori to the empirical (Immanuel Kant).

diamond strategies: double salto mortale from the theoretical to the hypertheoretical.

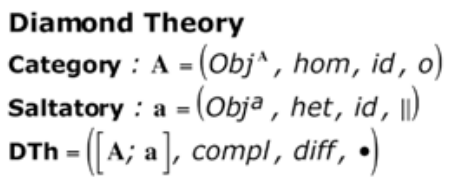

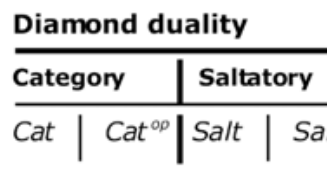

Categories are dealing with composition of morphisms and their laws. Saltatories are dealing with the jump-operation (saltisitions) of hetero-morphisms and their laws. Diamonds are dealing with the interplay of categories and saltatories. Their operation is interaction realised by the bridging operations. 
The laws of identity and associativity are ruling compositions, as well as saltisitions. Complementarity between categories and saltatories, i.e., between acceptional and rejectional domains of diamonds, are ruled by difference operations. Duality operations are applicable to both, categories and saltatories.

\section{Commutativity and associativity}

\section{Commutativity Condition}

If $f, g \in M C, l \in M C$ :

then

$g \Delta f=(g \circ f) \| I$

with $\left(\begin{array}{l}\omega(f) \Uparrow \alpha(g) \\ \operatorname{diff}(\alpha(g))=\alpha(l) \\ \operatorname{diff}(\omega(f))=\omega(l)\end{array}\right)$

such that

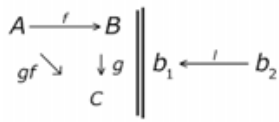

bi-commutes.

\section{Associativity Condition}

If $f, g, h, k \in M C$ and $l, m, n \in M C$ :

then

$(k \diamond h \diamond g \diamond f)=$

$\left[\begin{array}{l}k \circ(h \circ(g \circ f))=k \circ((h \circ g) \circ f) \\ ((k \circ h) \circ g) \circ f=(k \circ(h \circ g)) \circ f \\ (k \circ h) \circ(g \circ f)=k \circ(h \circ g) \circ f\end{array} \mid I\|(m \| n)=(I \| m)\| n\right]$

\subsubsection{Identity and difference}

"This shift becomes even more apparent if one examines the foundational concepts Nishida develops later in his career, the 'self-identity of the absolute contradiction' and the 'many in one, one in many' (tasokuitsu, issokuta); the former can be paraphrased as the 'identity of absolute difference' and the latter as 'plurality in oneness, oneness in plurality'." 34

34 Kopf 2004, p. 80 


\section{Identity and difference morphisms}

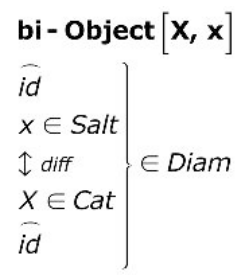

Identity is a mapping onto-itself as itself.

For each object $\mathrm{X}$ of a category an identity morphism, $\mathrm{ID}_{[\mathrm{X}, \mathrm{X}]}$, which has domain $\mathrm{X}$ in the category and codomain $\mathrm{X}$ in the same category exists. Called $\mathrm{ID}_{\mathrm{X}}$ or id $\mathrm{x}$ for $\mathrm{ID}_{[\mathrm{X}, \mathrm{x}]}$.

For each object $\mathrm{x}$ of a saltatory an identity morphism, $\mathrm{ID}_{[\mathrm{x}, \mathrm{x}]}$, which has domain $\mathrm{x}$ in the saltatory and codomain $\mathrm{x}$ in the same saltatory exists. Called $\mathrm{ID}_{\mathrm{x}}$ or $\operatorname{id}_{\mathrm{x}}$ for $\mathrm{ID}_{[\mathrm{x}, \mathrm{x}]}$.

Difference is a mapping onto-itself as other.

For each object $\mathrm{X}$ of a category a difference-morphism $\operatorname{DIFF}_{[\mathrm{X}, \mathrm{x}]}$, which has domain $\mathrm{X}$ in the category and codomain $\mathrm{x}$ in the saltatory exists.

For each object $\mathrm{x}$ of a saltatory a difference morphism, $\mathrm{DIFF}_{[\mathrm{x}, \mathrm{X}]}$, which has domain $\mathrm{x}$ in the saltatory and codomain $\mathrm{X}$ in the category exists.

This wording is a strict paraphrase of the common wordings of category theory. It also emulates its architectonics: from objects to morphisms to isomorphisms and to natural transformation, etc. Nevertheless it is not yet reflecting the reversed architectonics of the diamond way of thinking, where objects occur last and not first.

\section{Identity and difference composition}

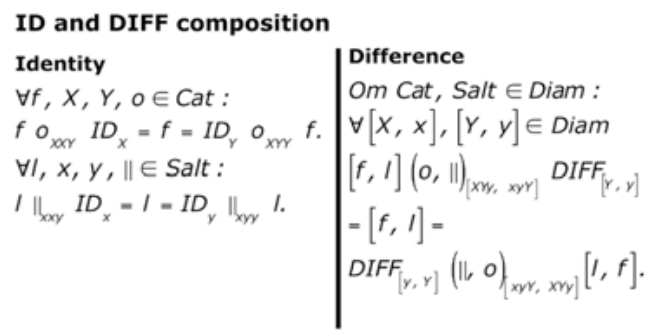




\subsubsection{Diamond concepts between iso- and xenomorphism}

"One philosophical reason for categorification is that it refines our concept of 'sameness' by allowing us to distinguish between isomorphism and equality." 35

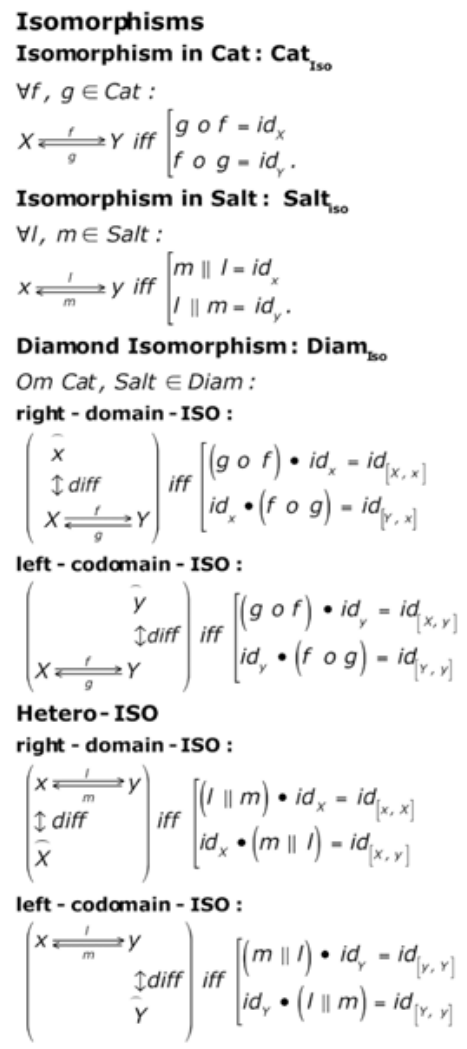

Category theory is studying, at first, isomorphisms between objects as domains and codomains of morphisms, then the trip goes on with functors, natural transformations and so on. Their basic element, thus, is an elementary, single morphism and their basic operation is a single identity morphism. Diamond theory is dealing with the interplay between categories and saltatories, hence, the elementary situation is not a single morphism but the interaction of the selected morphism and its two corresponding, i.e., interacting hetero-morphisms based on identity and difference operations. That is, the 
domain and the codomain of the selected morphism has to consider the corresponding domain and codomain of the hetero-morphisms involved. This is ruled by the difference operation.

Hence, the isolated objects as domains and codomains have to be supplemented by their own counter-parts, codomain and domain, to build their hetero-morphisms. In other words, the full interplay of morphisms, identity and difference mappings, has to be involved to realise proper diamond isoand xenomorphisms.

Full combined isomorphisms between morphisms and hetero-morphisms are naturally constructed out of the partial iso- and xenomorphisms. ${ }^{36}$

\subsubsection{Diamond concept of transversality}

A difference-philosophical interpretation of transversal isomorphisms could be found in the classical formulations of "The identity of oppositions, i.e., the identity of difference and identity." and "The difference of identity and difference". Both formulations are in some sense dual.

Further, more complex isomorphisms are easily composed by a combination of right- and left-isomorphisms.

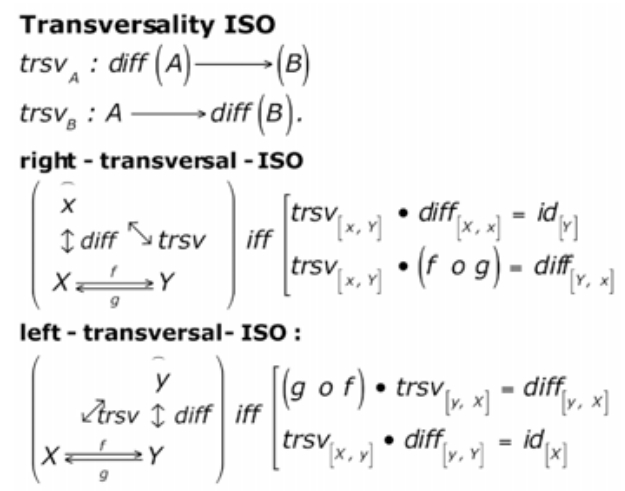

\subsubsection{Facets of diamond isomorphisms}

The concept of diamond isomorphisms is not solely dynamising the realm of sameness, as is the aim of category theory, but it is also inter-wined with the differentness and strangeness of otherness. ${ }^{37}$

36 Kaehr 2007a

37 Kaehr 2008a 


\section{Sameness (up to isomorphism)}

$$
\begin{aligned}
& \forall f, g, X, Y \in \text { Cat : } \\
& \widehat{X} \underset{g}{\stackrel{t}{\rightleftarrows}} \widehat{Y} \text { iff }\left[\begin{array}{l}
g \circ f=i d_{[x, x]} \\
f \circ g=i d_{[Y, Y]}
\end{array}\right.
\end{aligned}
$$

2. Differentness (up to xenomorphism) $\forall f, X, Y \in C a t, \forall I, x, y \in$ Salt :

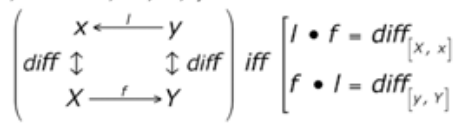

3. Strangeness (up to heteromorphism)

$$
\begin{aligned}
& \forall f, g, X, Y \in C a t, \forall I, m, x, y \in \text { Salt : } \\
& \left(\begin{array}{c}
x \underset{m}{\rightleftarrows} y \\
\downarrow d \text { diff } \\
X \underset{g}{\rightleftarrows} Y
\end{array}\right) \text { iff }\left[\begin{array}{l}
(g \circ f) \bullet(m \| I)=i d_{[x, x]} \\
(f \circ g) \bullet(I \| m)=i d_{[Y, y]}
\end{array}\right.
\end{aligned}
$$

\subsection{Interactionality as interplays in diamonds}

Interactionality of diamonds studies the interaction between disseminated categories and saltatories of polycontextural diamond systems. Given contextures in isolation, topics like duality and complementarity in diamonds are interactional, but they do not yet considering the inter-twining and intervening properties of interactivity as it happens with bridging. Thus, interactionality as an intra-contextural interplay occurs in elementary diamonds in forms of duality, complementarity, bridging and distributivity.

\section{Duality for Categories: "two for the price of one"}

The Duality Principle for Categories states

Whenever a property $P$ holds for all categories,

then the property $P^{o p}$ holds for all categories.

The proof of this (extremely useful) principle follows immediately from the facts that for all categories $\mathrm{A}$ and properties $\mathrm{P}$

(1) $\left(\mathbf{A}^{\mathrm{op}}\right)^{\mathrm{op}}=\mathbf{A}$, and

(2) $\mathrm{P}^{\mathrm{op}}(\mathbf{A})$ holds if and only if $\mathrm{P}\left(\mathbf{A}^{\mathrm{op}}\right)$ holds. ${ }^{38}$

Duality is defined for diamonds as duality of categories and duality of saltatories.

38 Adamek/Herrlich 2004, p. 27 


\section{Complementarity of formal languages}

The general principle underlying these limitations was called the linguistic complementarity by Loefgren. It states that in no language (i.e. a system for generating expressions with a specific meaning) can the process of interpretation of the expressions be completely described within the language itself. In other words, the procedure for determining the meaning of expressions must involve entities from outside the language, i.e. from what we have called the context. The reason is simply that the terms of a language are finite and changeless, whereas their possible interpretations are infinite and changing. ${ }^{39}$

The double meaning of diamond objects, bi-objects, is complementary and in their orientations they are not parallel but antidromic and deferred regarding the complementary system.

\section{Bridging categories and saltatories}

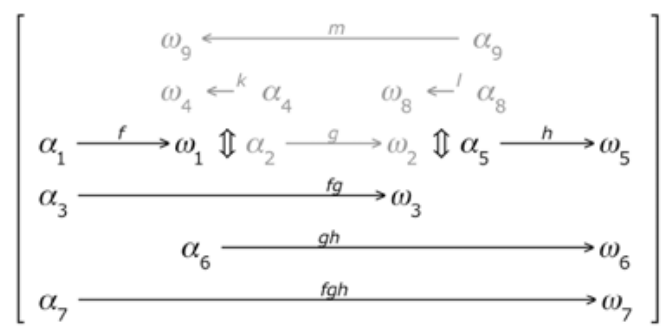

Bridging is not an operation of mediation or switching of and between diamonds or acceptional and rejectional actions in diamonds, but an operation to knot the two realms together, the categorical and the saltatorical. In the diagram, between the hetero-morphism $k, l$, the morphism $g$ is offering a bridge, marked in red, and thus interacting between the saltatorical and the categorical domain of the diamond. Complementarily, the two bridge pillars of the bridge are offered by the two hetero-morphisms $l, k$ defining the bridgework $g$. Thus, bridge and bridging are complementary actions, too. Both are reflecting the complementarity between categories and saltatories.

\section{Distributivity of composition, saltisition and bridging}

Because diamonds are based on interplays between categories and saltatories, which are involved with two fundamental operations: composition (o) and saltisition (||) with bridging $(\bullet)$ too, it is reasonable to find interactive 
laws as distributivity between those basic operators inside the very definition of the conception of diamonds.

\subsubsection{Duality in diamonds as duality in categories and saltatories}

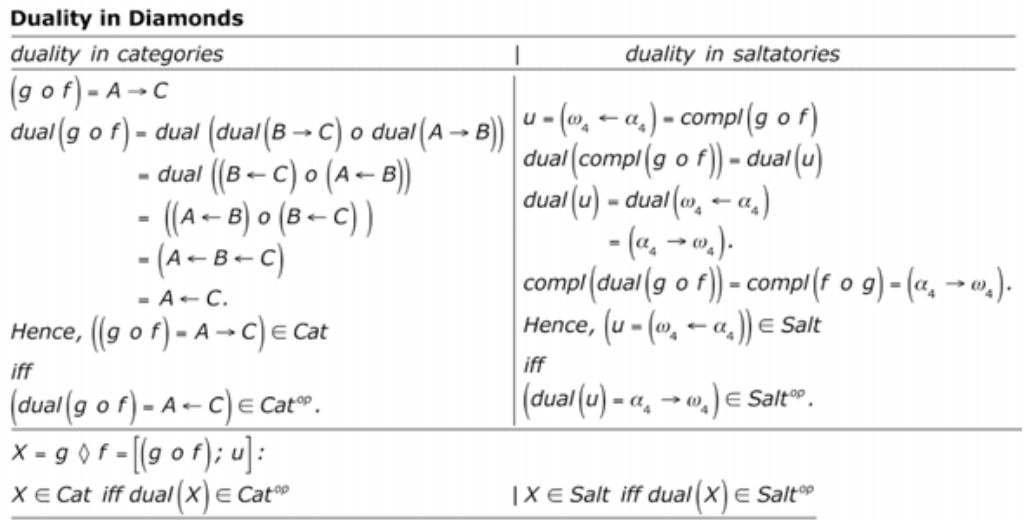

\subsubsection{Complementarity of categories and saltatories}

\section{Complementarity of Acc and Rej}

$x \in \operatorname{Acc}$ iff $\operatorname{compl}(x) \in R e j$

$X=g \circ f$ :

1. $x \in A c c$ if $\operatorname{compl}(x) \in R e j$

$\operatorname{compl}(g \circ f)=\operatorname{compl}(\operatorname{compl}(g) \circ \operatorname{compl}(f))$

$$
\begin{aligned}
& =\operatorname{compl}(\operatorname{diff}(\operatorname{cod}(f)) \circ \operatorname{diff}(\operatorname{dom}(g))) \\
& =\operatorname{compl}\left(\left(\overline{B_{c o d}}\right) \circ\left(\overline{B_{\text {dom }}}\right)\right)=\omega_{4} \leftarrow \alpha_{4} .
\end{aligned}
$$

$\left(u: \omega_{4} \leftarrow \alpha_{4}\right) \in R e j$

Hence, $(g \circ f) \in A c c$ if $\left(u: \omega_{4} \leftarrow \alpha_{4}\right) \in R e j$

$$
(g \circ f) \in A c c \text { if }(\overline{g \circ f}) \in R e j \text {. }
$$

2. compl $(X) \in \operatorname{Rej}$ if $X \in A c c$

$$
\begin{aligned}
\operatorname{compl}\left(\omega_{4} \leftarrow \alpha_{4}\right) & =\operatorname{compl}\left(\operatorname{compl}\left(\omega_{4}\right) \leftarrow \operatorname{compl}\left(\alpha_{4}\right)\right) \\
& =\operatorname{compl}\left(\left(A_{d o m} \rightarrow B_{c o d}\right) \leftarrow\left(B_{d o m} \rightarrow C_{c o d}\right)\right) \\
& =\left(\left(A_{d o m} \rightarrow B_{c o d}\right) \circ\left(B_{d o m} \rightarrow C_{c o d}\right)\right) \\
& =(f \circ g) .
\end{aligned}
$$

3. Hence, $x \in A c c$ iff $\operatorname{compl}(x) \in R e j$. 


\subsubsection{Bridging between categories and saltatories}

This new feature of bridge/bridging is ruling concrete intrinsic interactions.

\section{Bridging conditions and associativity for interactions}

\section{Bridge and Bridging Conditions BC}

1. $\forall k, l, n \in H E T, \forall f, g, h \in M O R P H$ :

a. composition

$g \circ f, g \circ h$,

$(h \circ g) \circ f, h \circ(g \circ f) \in M C$,

b. saltisition

$I\|k, n\| I$,

$n\|(I \| k),(n \| l)\| k \in \overline{M C}$,

c. bridges

$g \perp k, l \perp g$,

$(l \perp g) \perp k, l \perp(g \perp k)$ are in $\widehat{B C}$.

d. bridging

$g \cdot k, l \cdot g$,

$(l \cdot g) \cdot k, l \cdot(g \cdot k)$ are in BC.

2. $(g \bullet k) \in B C$ iff $\operatorname{dom}(k)=\operatorname{diff}(\operatorname{dom}(g))$,

$(l \bullet g) \in B C$ iff $\operatorname{cod}(I)=\operatorname{diff}(\operatorname{cod}(g))$,

$(l \bullet g \bullet k) \in B C$ iff $(g \bullet k),(l \bullet g) \in B C$.

3. $(g \perp k) \in \widehat{B C}$ iff $\operatorname{diff}(\operatorname{dom}(k))=\operatorname{dom}(g)$,

$(l \perp g) \in \widehat{B C}$ iff $\operatorname{diff}(\operatorname{cod}(l))=\operatorname{cod}(g)$,

$(l \perp g \perp k) \in \widehat{B C}$ iff $(g \perp k),(l \perp g) \in \widehat{B C}$.

\section{Bridging}

Assoziativity :

If $k, g, l \in B C$, then $(k \cdot g) \cdot l=k \cdot(g \bullet l)$,

Bridging :

bridging $_{(9,1, k)}: \operatorname{het}\left(\omega_{4}, \alpha_{4}\right) \bullet \operatorname{hom}\left(\alpha_{2}, \omega_{2}\right) \bullet \operatorname{het}\left(\omega_{8}, \alpha_{8}\right) \rightarrow \operatorname{het}\left(\omega_{9}, \alpha_{9}\right)$.

Bridge

Assoziativity :

If $k, g, l \in \widehat{B C}$, then $(k \perp g) \perp l=k \perp(g \perp l)$,

Bridge :

bridge $_{(0,1, k)}: \operatorname{het}\left(\omega_{4}, \alpha_{4}\right) \perp \operatorname{hom}\left(\alpha_{2}, \omega_{2}\right) \perp \operatorname{het}\left(\omega_{8}, \alpha_{8}\right) \rightarrow \operatorname{het}\left(\omega_{9}, \alpha_{9}\right)$.

Bridges vs. Bridging vs. Jumping

$(I \perp g \perp k) \triangleq(l \cdot g \cdot k) \triangleq(I \| k)$,

$(I \perp g \cdot k) \triangleq(l \cdot g \perp k) \triangleq(I \| k)$,

$(l \cdot g \perp k) \triangleq(l \perp g \cdot k) \triangleq(I \| k)$.

$\operatorname{diff}(\perp)=(\bullet),(\perp)=\operatorname{diff}(\bullet)$. 


\section{Bigraphs in diamond webs}

Instead of labelling transitions of the behavioural calculus, the whole system of bigraphs could be labeled (disseminated), i.e., distributed and mediated. Reflectionality between disseminated bigraphs, then might be realised by the "double-character" of diamonds. The possibility to disseminate bigraphs would open up a chiastic chain of connectivity and locality graphs, of statics and dynamics, as a new play of interactionality/reflectionality between bigraphical systems.

\subsection{Disseminated Diamonds}

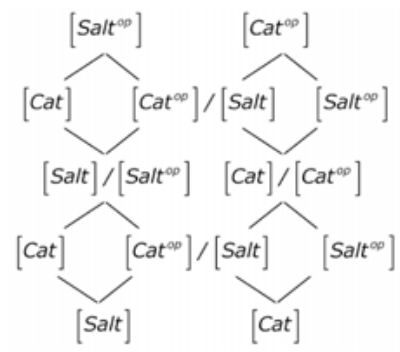

Diamonds, in this possible dissemination, are mapped as categories and saltatories with their dualities.

Mediation between diamonds happens horizontally, by complementarity and accretion from dual-categories to saltatories. And vertically, by duality and iteration from one diamond to another diamond of the grid. ${ }^{40}$

\subsection{Towards a diamond web of bigraphs}

In this setting we would have to introduce first the dual theory of bigraphs, which are themselves incorporating the dual structure of topography and connectivity. The more intriguing step would be to develop the complementary system to bigraphs and its duality, placed in saltatories. Both together are building the diamond of bigraphs, which then could be disseminated to model and design interactionality and reflectionality in a polycontextural system of interaction including the chiasm of global and local situations. Such a diamond web would not be restricted to informatic and physical global

40 Kaehr 2007c 
interactions like bigraphs but would be open to offer a framework for knowledge related semantic and pragmatic aspects of pluriversal computation and communication. Dissemination of diamonds might offer a scheme for a distribution and mediation of the orthogonality of connectivity and locality in bigraphs, which are themselves thematised as dualities. ${ }^{41}$

From a more futuristic vision, also with not much theory, Hai Zhuge (Beijing) develops the idea and sketches some steps towards a methodology of a knowledge grid, which is to "foster worldwide knowledge creation, evolution, inheritance, and sharing in a world of humans, roles and machines." ${ }^{42}$

\footnotetext{
41 Kaehr 2008b

42 Zhuge 2004, p. 1; see also Kaehr 2007e, f
} 


\section{References}

Adamek, Jiri/Herrlich, Horst/Strecker, George E. (2004): »Abstract and Concrete Categories. The Joy of Cats«. Online available: <http://katmat.math.uni-bremen. de/acc/acc.pdf> (last access: October 2007).

Baez, John C. / Dolan, James (1998): »Categorification«. Online available: <http: / arxiv. org/pdf/math.QA/9802029> (last access: October 2007).

Cheng, Eugenia/Lauda, Aaron (2004): »Higher-Dimensional Categories: an illustrated guide book«. Online available: <http://www.cheng.staff.shef.ac.uk/guidebook/ index.html> (last access: October 2007).

Cheng, Eugenia (2007): <http: / /www.youtube.com/TheCatsters> (last access: October 2007).

Heylighen, Francis (no year): »Making Thoughts Explicit: advantages and limitations of formal expression«. Online available: <http://pespmc1.vub.ac.be/Papers/Making Thoughts_Explicit.pdf> (last access: October 2007).

Kaehr, Rudolf (1996): Overview. <http://www.thinkartlab.com/pkl/PDF-Overview/ PDF-Overview.html>; <http: / / rudys-diamond-strategies.blogspot.com> (last access: October 2007).

Kaehr, Rudolf (2003a): »Strukturationen der Interaktion«. Online available: <http:// www.thinkartlab.com/pkl/media/SKIZZE-0.9.5-medium.pdf> (last access: October 2007).

Kaehr, Rudolf (2003b): »Zur Verstörung des (H)ortes der Zerstörung«. In: Kümmel, Albert/Schüttpelz, Erhard (Eds.), Signale der Störung, München: Wilhelm Fink Verlag, 119-138.

Kaehr, Rudolf (2004a): »Information is Information«. In: Variant 2(20), 25.

Kaehr, Rudolf (2004b): »Derrida’s Machines«. Online available: <http://www.thinkartlab.com/pkl/media/DERRIDA'S MACHINES.pdf> (last access: Ocotber 2007).

Kaehr, Rudolf (2005): »ConTeXtures. Programming Dynamic Complexity«. Online available: <http: / / www.thinkartlab.com/pkl/lola/ConTeXtures.pdf> (last access: October 2007).

Kaehr, Rudolf (2006a): »The Chinese Challenge: Hallucinations for Other Futures«. In: Variant 4(27), 37-38.

Kaehr, Rudolf (2006b): »From Ruby to Rudy. First steps ... to Diamondize Ruby«. Online available: <http://www.thinkartlab.com/pkl/lola/From Ruby to Rudy.pdf> (last access: October 2007).

Kaehr, Rudolf (2006c): »From Dialogues to Polylogues«. Online available: <http: / www. thinkartlab.com/pkl/lola/Games-short.pdf> (last access: October 2007).

Kaehr, Rudolf (2007a): »Steps Towards a Diamond Category Theory«. Online available: <http:/ /www.thinkartlab.com/pkl/lola/Diamond-Category-Theory.pdf> (last access: October 2007).

Kaehr, Rudolf (2007b): »Towards Diamonds«. Online available: <http:/ /www.thinkartlab.com/pkl/lola/Towards_Diamonds.pdf> (last access: October 2007). 
Kaehr, Rudolf (2007c): »How to Compose«. Online available: <http: / /www.thinkartlab. com/Chinese Challenge Pool/How_to_Compose.pdf> (last access: October 2007).

Kaehr, Rudolf (2007d): »Actors, Objects, Contextures, Morphograms«. Online available: <http: / /www.thinkartlab.com/pkl/lola/Actors+Objects.pdf> (last access: October 2007).

Kaehr, Rudolf (2007e): <http://www.youtube.com/watch?v=nOKj1yk9O4E> (last access: October 2007).

Kaehr, Rudolf (2007f): <http: / www.youtube.com/watch?v=jCNcFmPl-9E > (last access: October 2007).

Kaehr, Rudolf (2008a): »Which Equality? How equal are equal human beings?«. Online available: <http://www.thinkartlab.com/pkl/media/Equality/Equality.html> (last access: July 2008).

Kaehr, Rudolf (2008b): »Diamond Web 2.0? How social is social networking?«. Online available: <http://www.thinkartlab.com/pkl/media/Diamond_Web2.0/Diamond_ Web2.0.html> (last access: July 2008).

Kopf, Gereon (2004): »Between Identity and Difference. Three Ways of Reading Nishida's Non-Dualism«. Japanese Journal of Religious Studies 31(1), 73-103.

Milner, Robin (2004a): Axioms for bigraphical structure, Technical Report UCAMCL-TR-581, University of Cambridge, Computer Laboratory.

Milner, Robin/Jensen, Ole Høgh (2004b): Bigraphs and mobile processes (revised), Technical Report UCAM-CL-TR-580, University of Cambridge, Computer Laboratory.

Milner, Robin (2005): »Bigraphs: A tutorial«. Online available: <http: / /www.lix.polytechnique.fr/Labo/Robin.Milner/bigraphs-tutorial.pdf> (last access: October 2007).

Milner, Robin (2006): »Bigraphs: coordinating mobile computation«. Online available: <http: / /www.comp.leeds.ac.uk/siglac/Abstracts / leedstalk-milner.pdf> (last access: October 2007).

Milner, Robin (2007): »Pure bigraphs«. Online available: <http://www.cl.cam. ac.uk/ rm135/tutorial-7.pdf> (last access: October 2007).

Zhuge, Hai (2004): The Knowledge Grid, Singapore: World Scientific Publishing Co. 



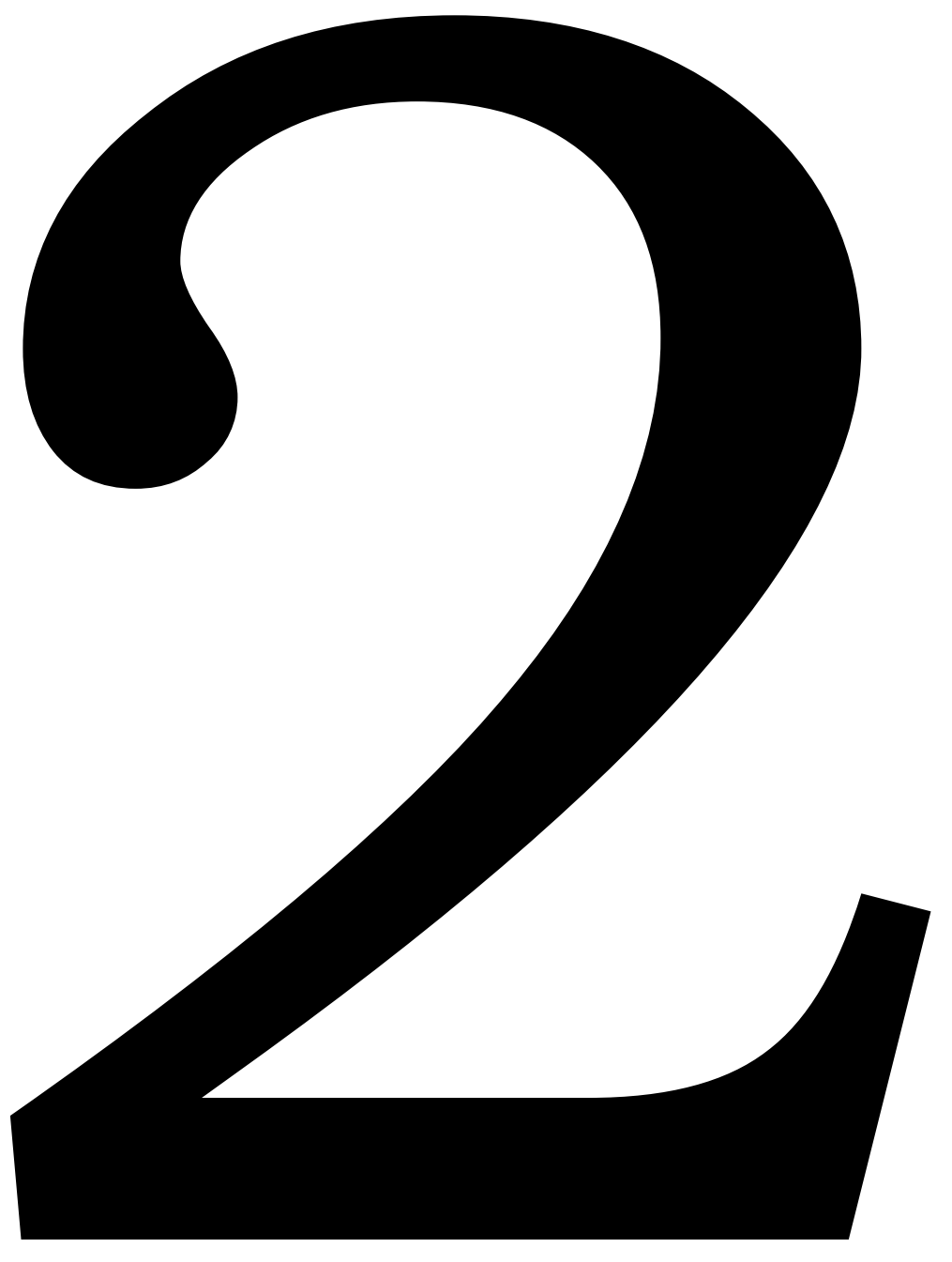




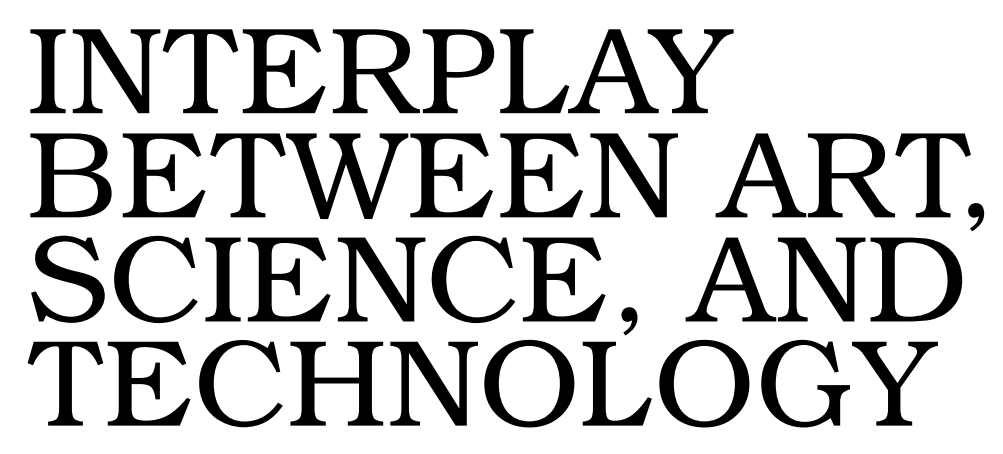




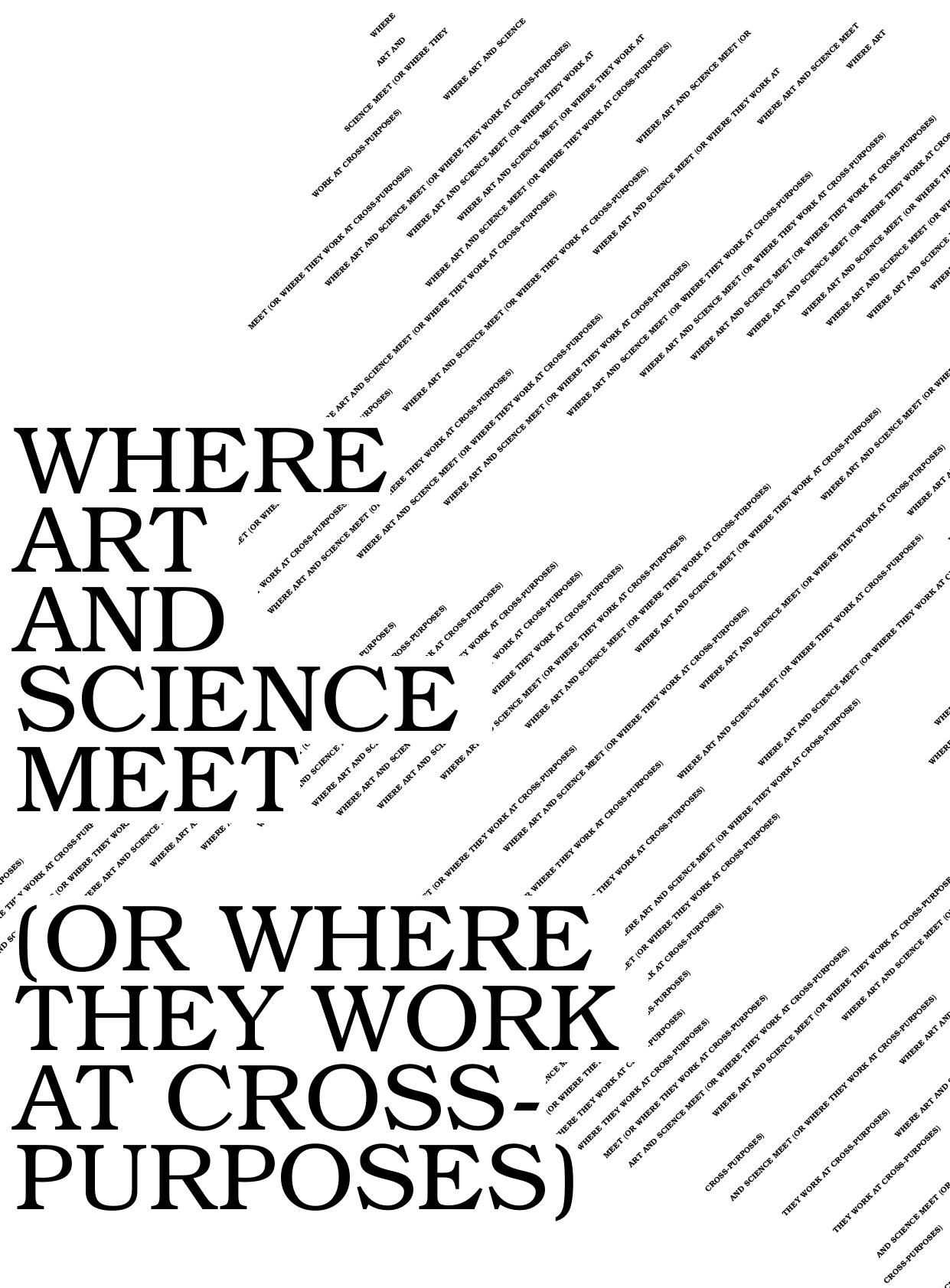

Hans H. Diebner 


\section{Attunement}

So-called new media art, occasionally referred to as "science art", undoubtedly creates paradoxes. It has repeatedly been pointed out that one of the main causes for these paradoxes lies in the "ontologische Indifferenz"1 of new media art as indicated by the oxymoron "science art". Is it science or art, both or neither of them? If a scientist (who without doubt is socialised differently from an artist) gets caught up in the maelstrom of media art and is keen on the new possibilities it offers, then she or he is at risk of being squelched under the wheel of the discourse as a result of the prevalent ambiguities. The convergence of art and science stimulates a kind of an immune system whose antibodies can be called destruction or deconstruction.

Interactive media art is systemic per se. With regard to this cybernetisation of art and science, a growing "affirmative negation logic" can be observed. System criticism becomes constitutive for knowledge and social systems that are criticised. Any escape seems difficult. On the one hand, art history predominantly wants to see the overcoming of the avant-garde's definition of art as general criticism. If, on the other hand, an artist addresses a scientific issue without explicitly criticising or satirising, then she or he is reproached for having an anachronistic recourse to a romantic concept of nature. Science in turn takes up avant-garde art's self-referentiality as essential for its own concerns and attempts to develop synergetic models of creativity from it that, in a sense, are composed of two antagonistic poles. ${ }^{2}$ This indicates exactly how the negative logic leads to the absorption of art into the system.

Annette Hünnekens and Claudia Giannetti are among the first who worked on a summary of hypotheses and a derivation of a theory for interactive art, respectively. ${ }^{3}$ Whilst Hünnekens discusses different artistic and theoretical positions and explains the underlying paradigms, Giannetti outlines an endo-aesthetics as part of digital aesthetics, which itself can be conceived of as paradigmatic. The endo-aesthetic concept directly follows Otto E. Rössler's endophysics, which is a natural scientific theory that radically renounces the subject-object distinction. ${ }^{4}$ Endophysics and its proximity to interactive art is controversially debated and serves as an instance of how transgressions between art and science can create enormous tensions in the

1 Hünnekens refers to a lack of distinction between art and science and speaks in this context of a "crisis of ontology" (Hünnekens 1997, p. 16). Mersch and Ott discuss the "historical differences and indifferences between art and science" (Historische Differenzen und Indifferenzen zwischen Künsten und Wissenschaften) (Mersch/Ott 2007, p. 9). I have chosen the term "ontological indifference" in order to refer to Heidegger's notion of "ontological difference".

2 Tröndle 2007, Tschacher / Tröndle 2005

3 Hünnekens 1997, Giannetti 2004

4 Rössler 1992

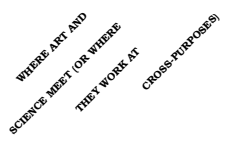


natural sciences, too. As a rule, however, border-crossers are barely recognised in the natural sciences. "Artists-in-Labs" 5 or similarly called programs have no corresponding "Scientists-in-Studios" programs or the like. Here the meta-level of reflexion is obviously attributed to art.

Within the science enterprise there does not seem to exist a larger need to reflect on the interrelation of art and science and thus about paradoxes, except for rhetorical reasons, as in: "we are also creative, so somewhat artistic after all." Often, a rudimentary understanding of aesthetics settles the matter, reducing art to the production of the "beautiful", since all the same "beauty" underlies scientific motives too. A second component of the scientific referring to art is its attempt to explain art as an emergent phenomenon within the scope of complexity theory, supporting its reduction to the "beautiful." Finally, art is seen in its functional role as a generator of creativity. ${ }^{6}$

In the following, starting out from existing perceptions amongst scientists of what the role of art is for science, I will try and work out a hypothesis on a paradox of interactivity based on Heidegger's use of the concept of reification (Verdinglichung). ${ }^{7}$ I will also pick up on some philosophical positions which attest the avant-garde stream to have contributed to their own absorption by science. According to Axel Honneth's interpretation, which comes close to Heidegger's application that I adopt here, Verdinglichung is a failure of Being (Seinsverfehlung). ${ }^{8}$ The ontological notion that "the world is a differential equation" is rarely expressed in such an explicit way, however, de facto implied in the scientific practice to a large extent. This can be considered as only one possible manifestation of Verdinglichung. The represented gets equated with the representation and, therefore, deprived of its existential quality.

Hünnekens has already pointed towards the difference which is reduced or missing due to interaction and by which some theoreticians even conclude that interactive installations cannot be art. I myself interpret this missing difference as an increase of Verdinglichung. This would abrogate Heidegger's distinction between science, that shows a tendency toward Verdinglichung, and art, which possesses a potential to wriggle out of Verdinglichung. This trend presents a certain consequence of the historical evolution of art. Above all, media art's proximity to technology and to topics of the natural and social sciences has convincingly been associated with a culmination of two tendencies that have been laid out by the avant-garde: its definition as a pure negativity as well as the integration of life into art. The result of this integration is precisely the indifference which should be conceived as a chance for the

5 Scott 2006

6 Tröndle 2007; Tschacher/Tröndle 2005

7 Objectification or reification are possible translations. I nevertheless prefer to keep the German expression in order to avoid blurring the meaning (Jahraus 2004).

8 Honneth 2005 
emergence of new cultural cornerstones from the perfect mixture, rather than as an occasion for polemics or perplexity.

The scientific tendency towards Verdinglichung - although not introduced as an essential concept in a Marxist context until Georg Lukacs ${ }^{9}$ - was critically addressed by philosophers of life in particular. ${ }^{10}$ Simply put, science reduces nature to its essences in terms of measurement or observational values. The technical applications of Shannon's information concept sharpened this tendency. In my judgement, Shannon's mathematical definition of information has commonly been generalised in an ill-considered way. One can speak of "datafication" or "cybernetisation" of life. As the complement of the essences ${ }^{11}$, the existences, can hardly be specified, the philosophy of life was often testified to have had a mystic character. Those scientists (e.g. Ilya Prigogine), operating on the edge of that mysticism, who want to dispense with the narrow systemic corset yet define themselves exact scientists rather than vitalists, are at a loss for explanations. ${ }^{12}$ This is mainly due to science's lack of a conception of time. Introducing the concept of the "existential", Heidegger created a philosophical framework within which that which (almost) defies discourse can nonetheless be thematised in a performative way. Jahraus speaks of an "auto-performance" 13 with respect to Heidegger's philosophy and relates it to the hermeneutic circle. A result of the effort not to fully detach science from Being is the incorporation of artistic degrees of freedom into the system theoretical approach. Exactly this, however, contributes to a kind of systemic conditioning of $\operatorname{art}^{14}$ and to the aforementioned indifference. The path of a performative science proposed here from the scientific perspective, attempts to abandon the representationalist reduction of art and rather highlight its performative power. What originates as a result of the adoption of performative concepts is not per se conceptualised as art, but merely an attempt, in the fashion of art, not to disregard existence (Dass-sein, cf. footnote 4). Contemporary art is no longer dominated by the paradigm of the avant-garde. According to my hypothesis that an increase of Verdinglichung results from the application of the avant-garde's conception of art especially with respect to its relation to science, this renunciation is welcome. However,

9 Honneth 2005

10 Amongst them, Henri Bergson $(1911,1948)$ counts to the most prominent because the importance of his work for contemporary system theory has repeatedly been stressed by Nobel prize winner Ilya Prigogine (1985).

11 "Essence" is a vexed issue. Scientists speak of grasping the "essence" when they attribute measures to something. Some philosophical streams regard "existence" as the "essence" of Being. I follow Heidegger who discriminates being in "Was-sein" and "Dasssein", i.e. "Wesen" and "Existenz" or essentia and existentia (Jahraus 2004, p. 193). In other words, "existence" is what fails when taking measures.

12 Holzhey 2004

13 Jahraus 2004, p. 193

14 Dammbeck 2007 
my provocative supposition is that the subversive avant-garde paradigm is not obsolete when being incorporated into scientific methodology.

\section{Retroactive Systems}

Hünnekens mentions, in passing, Hans-Peter Schwarz' suggestion of calling the new stream, "retroactive art". ${ }^{15}$ From a system-theoretical point of view the disappearance of this notion is regrettable. Systems are called "retroactive" if they include components that are capable of modelling those systems in order to enable the derivation of an intervention strategy from a simulation of that model.

Retroactive systems are thus subject to change exactly because we model them. They are dealt with in psychology, ecology, economics and sociology, to name but a few. In the course of their theoretical pervasion such systems create problems of self-referentiality that are the subject of both second order cybernetics and endophysics. It is retroactive systems in particular that render performative methods almost inevitable and provide an excellent reason to make methodological borrowings from retroactive arts. Being a disciple of Otto Rössler, my own transgression has been evoked through endophysics as well. The subversive idea of the brain's thermic noise entailing an uncertainty that projects onto the outer world is only one instance of a figure of thought that can be encountered in media art. For many years now, the media artist Bill Seaman has drawn explicitly on endophysics and has been cooperating with Otto Rössler. ${ }^{16}$

\section{Remarks on Verdinglichung}

Interactive media art draws per se on a cybernetic world-view. Recipients' measurable state variables are used to control the rest of the "machinic eigenworlds." Such an art is at a risk of increasing the degree of cybernetic Verdinglichung. Paradoxically, an interactive media installation offers science the possibility of reducing Verdinglichung. The integration of life into art or vice versa leads to a balancing act between contingency and habituation, between performativity and repetition.

Verdinglichung is seen here as a gradual property. ${ }^{17} \mathrm{I}$ assume that an absolute absence of Verdinglichung does not exist but can only be approxi-

\footnotetext{
15 Hünnekens 1997, p. 15

16 Seaman 2007

17 Note that Axel Honneth (2005) applies Verdinglichung only to extreme lapses of Being.
} 
mated. Heidegger operates with the notions of "present-at-hand (vorhanden; Vorhandenheit)" and "ready-at-hand (zuhanden; Zuhandenheit)" in order to emphasise the necessary detachment from ordinariness with respect to the appraisal of art. ${ }^{18}$ Interactive art is not only present-at-hand but also readyat-hand - in accordance with the avant-garde's demand to integrate life, but at the same time increasing the inclination towards Verdinglichung. I identify an entire Verdinglichung with the complete abdication of man's (capability of his) freedom of choice towards the machine. These are cases where - to put it crudely - the human is condemned to being a machine that merely nods things through.

\section{EyeVisionBot}

By means of "EyeVisionBot" (Fig. 1), an interface for image search, the above introduced line of thought can be demonstrated in an exemplary way. ${ }^{19}$ From a technical point of view the device consists of an eye-tracking unit, a database (potentially the www), a visual display and several computers that host the control software. The latter controls and analyses the gaze tracking, accesses the database, and steers the visual output. Simply put, the eye-tracking device detects the viewing direction of the user. With this it is possible, given a display of twentyfive images arranged in a $5 \times 5$ matrix, to determine which images are looked at and for how

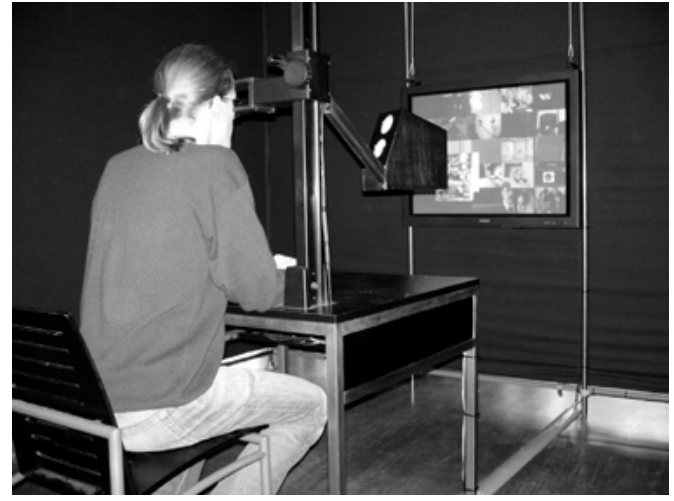

Fig. 1. EyeVisionBot long (Fig. 2a-b). Those images being momentarily looked at become slightly magnified in order to give visual feedback. After a certain time, five times five new images are retrieved from the database and shown on the display. This

18 Jahraus (2004, p.79) summarises Heidegger's thoughts on art as following: "Umgekehrt aber sieht er in der Kunst einen ästhetischen Ausdruck dessen, was die Technik gerade vergessen macht: die Teilhabe am Sein. [...] Während Heidegger an der Technik Verdinglichungstendenz metaphysischen Ausmaßes herausarbeitet, soll die Auseinandersetzung mit der Kunst gerade dazu dienen, diese Verdinglichungstendenz zu überwinden."

19 Fischer et al. 2005 
time, instead of a random selection (as was the case with the initial access), the detected gazing-durations are used to pre-estimate the most desired categories, which are then preferentially accessed. A category is defined by potential classifications applied to the database and structural similarities of the images. Given a non-classified database, the search can be made on the basis of structural relations alone. The structural comparison is conducted with the open source software GIFT (GNUImage Finding Tool).

In a museum installation (Fig. 1) we used the image database of the "media art net" project. ${ }^{20}$ If, for example, out of the first 25 presented images, a photograph of a media installation is looked at for a notably long time, then the user may be interested in this style of installation, have a particular interest in the corresponding artist or may be looking for another image that resembles the one she or he singled out. Several models that can be used to control the subsequent search are possible. The assumption underlying those models is that the momentarily presented 25 images' competition for the user's attention would eventually lead to a distribution of gazing durations which reflects the user's priority distribution with regard to the corresponding categories. A universal algorithm for modeling and simulation of such subjective weighting in decision-making processes is based on Bayesian inference. It involves continuous re-weighting of possible hypotheses on the basis of given observations. Since it is usual, during each 'turn', for more than one image to be regarded for different periods of time, under certain conditions one can quite robustly estimate the desired categories within one cycle. The quota of the subsequent turn's categories is calculated proportionally to the gazing durations. A precondition for an efficient adaptation is a definitely fixed task such as, for example, the search for a specific image whose appearance is roughly memorised but for which neither the artist nor the style can be recalled. The structural resemblance then leads quickly to success.

We originally proceeded on the assumption that the interface, along with further developed software, would constitute a creative tool for establishing dynamic user-generated database ontologies. However, it became evident that user modeling merely maps the set of prejudices onto itself. In other words, the interface as originally conceptualised only functions in a satisfactory way if a relatively precise aim governs the search.

Assuming a perfect adaptation of the system to the user's preferred categoryin interaction with the user, the system will always stay in this category and without a special interference - like randomly adding images out of arbitrary image categories - a change to another category will be impossible. For considerably more complex decision-making processes like in medical diagnostics for which the user's preferences are automatically anticipated by the

20 Frieling/Daniels 2004; 2005 


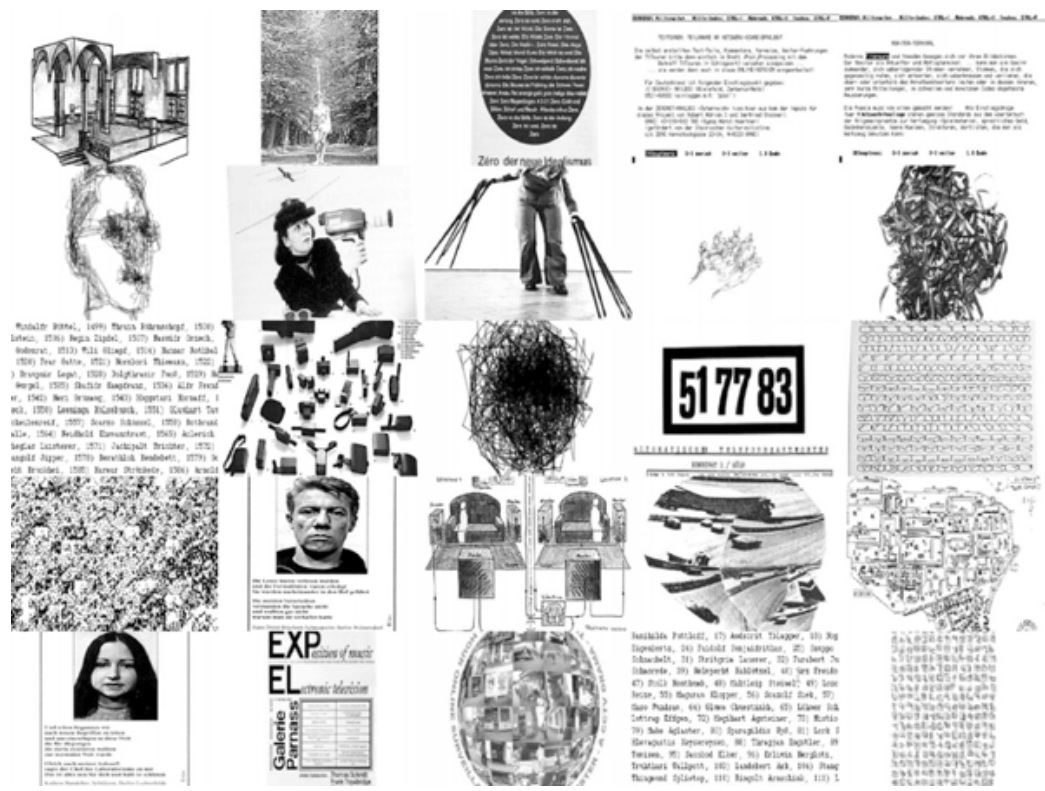

Fig. 2a. First array of presented images. The momentarily watched image is slightly magnified.

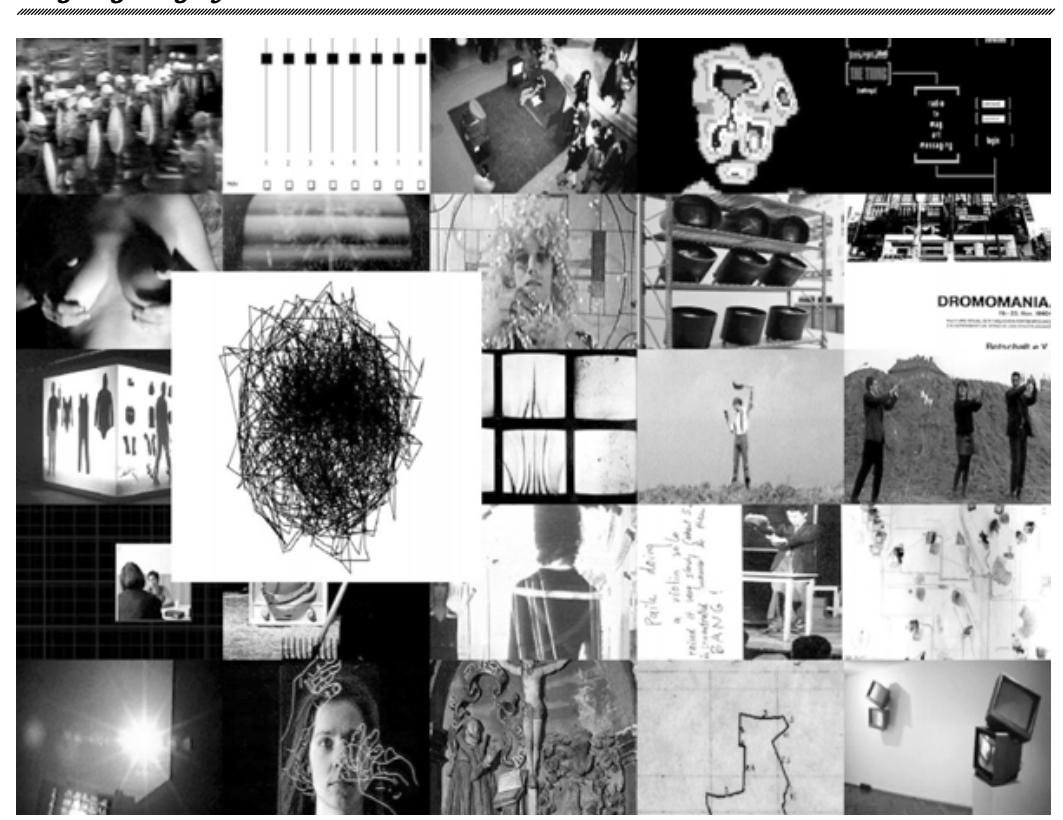

Fig. 2b. Second array of presented images indicating structural and taxonomic similarity. 
algorithmic system, suspicions could be raised that a kind of "self-imposed nonage" of the user sets in as a result of taking pleasure in the release of cognition. If, in addition, the decision-making process is one of high responsibility ${ }^{21}$ then - according to my suspicion - the people will surrender themselves to the "objective" algorithmic decision for convenience. In other words, what was intended as backing for decision-making ends up in transferring the decision along with the "responsibility" to the algorithm. Human error turns into technical failure. This would then be high-degree Verdinglichung.

Therefore, with regard to creative applications it seems to make more sense to investigate different deviations from the optimal user model. However, there are no rules as to how to achieve this. Only a causally open system can be creatively utilised. In my opinion, however, it is possible to generate an understanding of the mechanisms behind the user's handling by means of performatively approached "museum field studies" without being able to directly translate these mechanisms into a definite mathematical model. Furthermore, a museum installation functions as a critical interface..$^{22}$ One might, for example, employ EyeVisionBot to scrutinise one's own habits by uncovering the normally invisible algorithmic decision processes. The lack of a significance value certifying the tool to be more efficient than other methods for particular tasks so far hindered any publication in a professional journal. Therefore, the museum seems to be both the genius loci for performative scientific studies and a means for its publications, something which EyeVisionBot is intended to give an example of. A crucial point is the physical presence of the person whose cognition is to be understood partially. What is being modeled becomes part of the model.

One could have the suspicion that due to inevitable user-modeling, interactivity has a tendency toward Verdinglichung, but can at the same time avoid complete Verdinglichung as long as the users are allowed to be physically involved.

\section{Remarks on the Bayesian Algorithm}

Until recently the aforementioned Bayesian algorithm was mainly used in medical diagnostics. This method for the estimation of the validity of hypotheses integrates current investigation results and prior knowledge. It is heavily criticised by some statisticians because the recourse to prior knowledge is equated with a dependency on prejudices. Subjective degrees of reliability are described by it. For only the last few years, however, the estimation of subjective probabilities is emphasised as an advantage of the method, namely in

21 E.g., the correct categorisation of an X-ray image through a physician.

22 Pold 2005 
cases of anticipating human decisions. With it, subjective decision making processes can be quasi objectified. Software that we use on a daily basis contains context-sensitive, cognition-supporting algorithms that are identical or very similar to the Bayesian method. Examples are junk mail recognition or the Office assistant. Computer-based surveillance and control processes contain these algorithms. "Semantic" search engines are based upon related methods. Brain physiologists even claim that during our decision-making processes a Bayesian algorithm is executed. ${ }^{23}$ Therefore, the method has already been compared with the hermeneutic circle $^{24}$ giving rise to the formulation of a "Bayesian Epistemology". ${ }^{25}$ In my opinion, this is a categorical mistake. With the aid of the Bayesian inference principle each decision-making process can be approximated, and this can be carried out more effectively the more it is based on invariants. New ideas, however, mostly originate from "irrational" decisions that the algorithm is unable to describe and cannot therefore simulate. The power of simulations lies in the description of stationary systems rather than in contingent ones.

Recently, a further step in objectification has been discussed in medicine as well as numerous other disciplines (Law, History, Economics, and others) under the heading of "evidence-based medicine". There are database projects which allow for the retrieval of all accumulated previous decisions for the purpose of obtaining comprehensive and robust estimations of a priori probabilities. Occasionally, for reasons of objectivity, relinquishing the decisions based on the database content and its algorithmic evaluation to the algorithm itself becomes necessary. In this way, artificial intelligence enters a causally closed sphere, thereby degrading human decision-making, in much the same way as an epiphenomenon, to a nodding-through farce. The modelling of retroactive systems in such a way (that allows for an anticipation of decisions respective of the activities of agents so efficiently that the latter readily accept the results), would mean a high degree of Verdinglichung. In the following, I wish to argue that with respect to works that are motivated by a putative emancipation of society, media art is at risk of co-designing such an "evidence-based" society.

23 Rao 2005

24 Mallery et al. 1987

25 Bovens/Hartmann 2003 


\title{
Integration of Avant-garde into the System
}

As mentioned above, retroactive art (science art) and the convergence of cultures that is generally discussed under the label of "Art \& Science" is considered a logical consequence of the avant-garde movement. Boris Groys (2005) elaborates on this:

\begin{abstract}
Since the 1970s we have been living and functioning in a post-revolutionary system of art. According to G.W.H. Hegel (1770-1851), all post-revolutionary societies are characterised by the fact that they prescribe rational goals, procedures and strategies to their members, and demand explanations, justifications and precise plans from them. It is obvious that our present art system functions precisely according to these rules. The claim of a single artist that his or her work is an unpredictable, creative act, seems obsolete, and is not taken seriously by today's art world. [...] it was precisely the radicalisation of the notion of creativity by the revolutionary avant-garde that has historically led to its integration into the 'system'. The avant-garde art saw itself as the embodiment of the pure negativity, as the medium of destruction and annulment of all traditional, mimetic, naturalistic art forms.
\end{abstract}

The basic statement of "integration into the system" is affirmed by Dieter Mersch and Michaela Ott (2007) as well as Gerhard Gamm (2007). The aforementioned authors emphasise the role of cybernetics in this context. A system theory that pretends to include epistemological processes in its models and simulations almost necessarily presents an attractor for artists who have always been endophysicists in their self-conception long before the notion of endophysics was coined. In the year 2003, Lutz Dammbeck (2007) who is an artist-scientist and hence, like nearly everyone, a theoretician too, with his documentary "Das Netz" (The Net), began to discuss the role of art within the cybernetic world conception on a meta level. In a recent article entitled "Re-Reeducation or: Art and Conditioning" he speaks alternately of a "digital dictatorship" resp. a "systemic dictatorship." He repeats the position (already mentioned a number of times) that the avant-garde contributed to its own absorption into the system through its categorical system criticism. Dammbeck conceives the assimilation of art into the system in such an extensive way that having read his article one has to wonder whether there was any art after World War Two that was not engrossed in the system or found its legitimation exclusively from the system.

Dammbeck fears a global brainwashing, and Pavlovian conditioning in which artists only have to play the "criticising class clown". He says that it can be clearly seen

that the idea of an 'outside' from which the 'inside' can be changed is naive in the face of patterns and structures designed by cybernetics and system theory, because each point at the periphery is at the same time the center and an 'outside' no longer exists. And we also know: the mere thought of 
a possible change produces an energy that can be used by the system in the same way as every attack or perturbation as an intake of energy for further perfectioning. (...) Therefore, it would be meaningless to take action against it, since each critique not only preserves the system's life but even strengthens it. Metaphorically speaking: Those who touch the machine are already part of it and its codes.

In other words, after the cybernetic conception of nature, a systemic role was assigned to everything. I do not regard it as impossible that we are dealing with a kind of brainwashing paranoia which was called "cybernetic irony" by Peter Sloterdijk in a conference on the film "Matrix" (Sloterdijk 2000). What is noteworthy in this context is the video installation entitled Psych|OS, belonging to the distinguished actionist collective "Übermorgen". The entirely confusing recordings were made by one of the members of the actionist group, Hans Bernhard, during his stay in a psychiatric hospital due to a serious psychosis. One should know that Übermorgen belong to the most effective system critics. Hans Bernhard, for whom the quarrel with "the net" is a kind of a self-therapy, writes about himself and "the net": ${ }^{26}$

\begin{abstract}
Hans Bernhard's neuronal networks are connected to the global network, and his mental illness - the bipolar affective disorder that in March 2002 sent him to a mental hospital - is the network's illness. The video called Psych|OS (2005) sums up this experience, in which those two levels - digital and real, bio \& tech, nervous system and operative system - merge. This nervous system, infected by the hi-tech, needs a treatment, and the hi-tech society prescribes its remedies, bio-chemical 'agents' which control the internal information flow. [...] The Psych|OS Generator (2006) is the literal application of this kind of control: a piece of software that asks the user about the symptoms of her disease and provides her with a remedy, in the form of a 'forged original' medical prescription.
\end{abstract}

\title{
Viral Dynamics
}

Within the area of "street-art" Julia Reinecke affirms the ontological ambiguity as a consequence of the avant-garde. ${ }^{27}$ Here, it is the indifference between art and commerce. The relation this bears to the topic discussed is closer than it might be expected.

Street-art is a form of actionism and in some respect is comparable with hacktivism, i.e. Internet actionism. Most street-art activists do not call themselves artists, yet locate themselves within the tradition of situationism and other streams of avant-garde art which dedicated themselves to the integration of life into art. Street-art explicitly locates itself between system critique and system conformity. This leads to a continuous innovation with respect to commercial trends, but this innovation is itself subsumed in commercial

26 Übermorgen 2005

27 Reinecke 2007 
trends once again at a tearing speed. Interestingly, the activities are often fully detached from content. Similar to situationism activities are undertaken purely for their subversive or provocative impact. The whole art of actionism consists only in the strategy. Unsurprisingly, the relatively young commercial movement called Guerilla marketing evolved directly from street-art whose main concern is attention economy, and where product information is pushed to the subliminal border. Guerilla marketing has recently re-implemented itself into street-art as Guerilla art, where quite frequently the artistic content is crowded out to the periphery in an analogous way. Many activities in Guerilla art and Guerilla marketing are conceptionally indistinguishable. For instance, within the "go public" actionism of Michael Bielicky there is, in the first instance, no talk of the content of the art emerging in a Guerilla-like way in public space. ${ }^{28}$

The mechanisms of the propagation of "signifiers without signified" that underlie Guerilla marketing led to the related concept of viral marketing. ${ }^{29}$ This concept assumes the viability of modelling word-of-mouth propaganda as epidemiological dynamics. In this way, two previously autonomous currents in dynamic models of cultural evolution converge: memetics and marketing. Memetics claims to generalise Darwinian theory of evolution and to be capable of describing cultural evolution by virtue of comparable mechanisms. ${ }^{30}$ Corresponding to genes, the basic units of culture are memes, which spread and survive according to the laws of selection of the fittest. The concrete propagation dynamics is equivalent in its form to the proliferation of viruses, giving rise to the name "viral marketing". Similar to genetic engineering, the concept of viral marketing assumes that specific phenotypes can be designed. This is particularly easy to do on the Internet, because easily accessible information (tags, newsfeeds, access statistics, memes detectable via data mining) on bloggers' habitus in the subcultural field, the "blogosphere", can be used to monitor, model and design this part of society. Here, epidemiological dynamics are coupled with graph theoretical models from network theory. By now, it is possible in some simple cases to calculate optimal conditions for meme propagation. Great efforts are being made to improve this analysis of structures and life-cycles of memes by employing pseudo-hermeneutic Bayesian statistics.

Similar to tags and stencils in street-art, viral marketing (with the collaboration of artists) inoculates the virtual world of the Internet with memes (videos, flash animations, games, etc.) that tout for attention. The strategy of viral marketing makes itself the subject of discussion, and so it is, to an 
amazingly high degree, effectively self-promotioning. This is a typical "line of argument" corresponding to "cybernetic irony."

One noteworthy system is the monitoring system "Blogviz"31 with which the flow of memes in the blogosphere can be monitored. The corresponding Master's Thesis contains a detailed chapter on previous artistic achievements. As a particular example, I'd like to mention the prizewinning installation "Listening Post" by Mark Hansen and Ben Rubin. The following can be read online on this installation: "Listening Post is an art installation that culls text fragments in real time from thousands of unrestricted Internet chat rooms, bulletin boards and other public forums. The texts are read (or sung) by a voice synthesizer, and simultaneously displayed across a suspended grid of more than two hundred small electronic screens." This work is mentioned in several other publications on memetics (which cannot be listed here) as an important ground-preparing work. As an example it may suffice to show how memetic research and avant-garde art and, for that matter, "datafication" and "cybernetisation" of Being are interwoven, especially since this art is not only the subject of memetic modeling, but also, as in the case of "Listening Post", develops memetic models by itself and designs them in a sensual way.

\section{Remarks on Cybernetic Irony}

The works of net activists often are of an intensely paradoxical form. In the case of "Amazon Noir”, Übermorgen hacked online bookseller Amazon's web presence in order to apply an efficient algorithm providing access to sample pages that could be combined to complete books. Manually, this would take month or years. Amazon became aware of the hack and put Übermorgen under pressure. Eventually, Übermorgen sold the algorithm to Amazon and signed a non-disclosure agreement. The campaign is now exhibited in a purely symbolic way. Übermorgen (Ü) was interviewed on this matter by the online journal Telepolis (T): ${ }^{32}$

Ü: Our projects are purely about experimenting: Amazon Noir is not a statement on copyright and even less an attack on the online trader. There is no specific goal behind it; the matter simply arose. I call it freestyle basic research. We build a setting and observe what happens sociologically, with relation to mediation, and technologically. We didn't have a fixed plan of an outcome at the time. The sell-off arouse as a new solution, and so we opted for the agreement with Amazon.

$\mathrm{T}:(\ldots)$ there is no comment at all by Amazon on the alleged sale of the software. De facto the whole action might just as well not have happened and be merely merged. (...) Wouldn't this be the "next level" in media hacking: coverage of actions that have never happened at all?

31 Lima 2004

32 Pettauer 2006 
Ü: Of course we already did such things and we experiment with it, but in our big projects like Google Will Eat Itself and Amazon Noir it is essential that the technological part functions (...). We are lazy-bones and it is tedious having to make up everything!

One can find an almost indefinite number of similarly absurd performances, which leave the matter unclear as to whether they are viral marketing activities, hacking or simply cybernetic irony. Incidentally, in the case of Dammbeck's prognosis, the question of whether one should regard the spectre of a global cybernetic brainwashing as similarly being an artistic concept or as being a serious contribution to media theory, is virtually irrelevant with respect to its ironic impact.

\section{Back to Earth}

According to Mersch and Ott avant-garde, which to a great extent referred to Nietzsche, is a "reciprocal radicalization that accepted the challenge, not only to hold it's own ground with respect to the sciences but to eventually imbibe them." ${ }^{33}$ According to Nietzsche, Mersch and Ott elucidate, artistic practice is the "Ereignung von Ex-sistenz" ${ }^{34}$ itself. "Of a higher sense than any discourse, art literally reaches down to the abysms of Being.", they further explicate. Actually one is inclined instead to diagnose art has having been merged into science, albeit in a manner that is just opposed to the "Enowning of Ex-sistence". I therefore take Dammbeck's diagnosis of the artist as a "critical class clown" very seriously, but I do not share his pessimistic stance. It is now important not to repeat the mistakes made by the Frankfurt School, (who took a generally pessimist stance), but rather to undertake a critical but constructive approach.

In my opinion, the necessity almost inevitably follows from this to provide sciences with an understanding of the "existential" and the attempt to transfer the original avant-garde criteria to sciences, namely to integrate life, i.e. to create a causally open structure. It should be made possible for "agent causality" to be brought into the system. It obtains performativity, the enowning of existence is enabled.

The lack of understanding among scientists usually created by recourses to existential philosophy such as "Enowning of Ex-sistence" usually came to light in the dispute between Ilya Prigogine and Jean Bricmont. Prigogine, who based his ideas on Henri Bergson's process philosophy, was accused first by Bricmont (1995) and then additionally by Alan Sokal (Sokal/Bricmont 2001)

\footnotetext{
33 Mersch/Ott 2007, p. 17

34 "Enowning of Ex-istence", following Heidegger.
} 
of abusing science. Not many attempts to change scientific methods and the conception of time such as that of Prigogine come from within natural science. Therefore, it makes sense to demand an intake of the concept of the existential into a science which is restricted to essentials. It is exactly this domain where participative, interactive, retroactive, performative or however described media installations abundantly endow a great deal of sense. Science receives corporeality, and reality becomes rehabilitated in a certain sense. I suggested that such a repertoire of methods, enriched with the existential, should be called "performative science". ${ }^{35}$ From this perspective it follows that art is not reduced to a functionality in commission of science, indeed not to a functionality at all, and that the development of art in emancipation from science can even be advocated. However, it is mandatory to accept a new episteme that settles between traditional science and art.

\section{References}

Bergson, Henri (1911): Creative Evolution, London: MacMillan \& Co.

Bergson, Henri (1948): Denken und schöpferisches Werden, Meisenheim am Glan: Westkulturverlag Anton Hain.

Bielicky, Michael (2007): »Go Public / Guerilla Screening«. Online available: <http:// solaris.hfg-karlsruhe.de/hfg/inhalt/de/Presse/Presseinformationen /Februar\%20 07/06_2007> (last access: October 2007).

Bovens, Luc/Hartmann, Stephan (2003): Bayesian Epistemology, Oxford: Clarendon Press.

Bricmont, Jean (1995): »Science of chaos or chaos in science?«. Physicalia Magazine $17,159-208$.

Dammbeck, Lutz (2007): »Re-Reeducation oder: Kunst und Konditionierung«. Online available: <http://www.heise.de/tp/r4/artikel/26/26380/1.html> (last access: October 2007).

Dawkins, Richard (1996): Das egoistische Gen, Reinbek: Rowohlt.

Diebner, Hans H. (2006): Performative Science And Beyond - Involving the Process in Research, Wien: Springer.

Fischer, Sebastian/Scherffig, Lasse/Diebner, Hans H. (2005): »EyeVisionBot«. In: Bruno Latour/Peter Weibel (Eds.), Making Things Public - Atmospheres of Democracy, Cambridge: MIT Press, 1017.

Frieling, Rudolf/Daniels, Dieter (2004): Medien Kunst Netz 1: Medienkunst im Überblick, Wien/New York: Springer.

35 Diebner 2006 
Frieling, Rudolf/Daniels, Dieter (2005): Medien Kunst Netz 2: Thematische Schwerpunkte, Wien/New York: Springer.

Gamm, Gerhard (2007): »Vom Wandel der Wissenschaft(en) und der Kunst«. In: Dieter Mersch/Michaela Ott (Eds.), Kunst und Wissenschaft, München: Fink, 35-51.

Giannetti, Claudia (2004): Ästhetik des Digitalen - Ein intermediärer Beitrag zu Wissenschaft, Medien- und Kunstsystemen, Wien: Springer.

Groys, Boris (2005): »The Mimesis of Thinking«. In: Donna De Salvo (Ed.), Open Systems - Rethinking Art c.1970, London: Tate Publishing, 50-63.

Hermann, Frederik (2004): Virales Marketing. Diploma Thesis, University of Karlsruhe.

Holzhey, Christoph (2004): »Self-Organization at the Edge of Mysticism«. Online available: <http://www.mystik.uni-siegen.de/Texte/Holzhey2004a.pdf> (last access: June 2008).

Honneth, Axel (2005): Verdinglichung, Frankfurt a. M.: Suhrkamp.

Hünnekens, Annette (1997): Der bewegte Betrachter - Theorien der interaktiven Medienkunst, Köln: Wienand.

Jahraus, Oliver (2004): Martin Heidegger - Eine Einführung, Stuttgart: Reclam.

Lima, Manuel (2007): »blogviz - Mapping the dynamics of information diffusion in blogspace«, Master's Thesis. Online available: <http://www.blogviz.com> (last access: October 2007).

Mallery, John C./Hurwitz, Roger/Duffy, Gavan (1987): »Hermeneutics: From Textual Explication to Computer Understanding?«. In: Stuart C. Shapiro (Ed.), The Encyclopedia of Artificial Intelligence, New York: John Wiley \& Sons, 596-611.

Mersch, Dieter/Ott, Michaela (2007): »Tektonische Verschiebungen zwischen Kunst und Wissenschaft«. In: Dieter Mersch/Ott, Michaela (Eds.), Kunst und Wissenschaft, München: Fink, 9-31.

Pettauer, Ritchie (2006): »Geistiges Eigentum rekombinieren. Ein Gespräch mit Hans Bernhard von Ubermorgen.com über die Aktion >Amazon Noir«. In: Telepolis, <http: / / www.heise.de/tp/r4/artikel/24/24034/1.html> (last access: October 2007).

Pold, Søren (2005): »The critical interface«. In: Olav W. Bertelsen/Niels O. Bouvin/ Peter G. Krogh/Morten Kyng (Eds.), Critical Computing - Proceedings of the 4th Decennial Conference on Critical Computing: Between Sense and Sensibility, Aarhus, Denmark/New York: ACM Press, 109-112.

Prigogine, Ilya (1985): Vom Sein zum Werden, München: Verlag Piper \& Co.

Rao, Rajesh P. N. (2005): »Hierarchical Bayesian Inference in Networks of Spiking Neurons«. Advances in Neural Information Processing Systems 17 [= Neural Information Processing Systems, NIPS 2004, December 13-18, 2004, Vancouver, British Columbia, Canada], 1113-1120.

Reinecke, Julia (2007): Street-Art: Eine Subkultur zwischen Kunst und Kommerz, Bielefeld: transcript.

Rössler, Otto E. (1992): Endophysik: Die Welt des inneren Beobachters, Berlin: Merve. Scott, Jill (Ed.) (2006): Artists-In-Labs: Processes of Inquiry, Wien: Springer. 
Seaman, Bill (undated): »Endophysics and The Thoughtbody Environment - An Outline for a Neo-Computational Paradigm«. Online available: <http://digitalmedia.risd. edu/billseaman/pdf/tb_endoNeo-1.pdf> (last access: October 2007).

Sloterdijk, Peter (2000): »Kybernetische Ironie - Die Philosophie der Matrix«. Online available: <http://www.societyofcontrol.com/library/theory/sloterdijk_matrix.txt> (last access: October 2007).

Sokal, Alan / Bricmont, Jean (2001): Eleganter Unsinn - Wie die Denker der Postmoderne die Wissenschaften mißbrauchen, München: Dtv.

Tröndle, Martin (2005): »Das Orchester als Organisation: Exzellenz und Kultur«. In: Timo Meynhardt/Ewald J. Brunner (Eds.), Selbstorganisation managen - Beiträge zur Synergetik der Organisation, Münster: Waxmann, 153-169.

Tschacher, Wolfgang/Tröndle, Martin (2005): »Die Funktionslogik des Kunstsystems - Vorbild für betriebliche Organisation?«In: Timo Meynhardt/Ewald J. Brunner (Eds.), Selbstorganisation managen - Beiträge zur Synergetik der Organisation, Münster: Waxmann, 135-152.

Übermorgen (2005): Psych|OS. Video-Installation. Online available: <http://www.resqualia.net/view_projecte.php?id=441\&PHPSESSID=6fd735da692d7cfbe $11340 \mathrm{f} 643$ acc81d> (last access: October 2007). 


\section{TIME,}

MAGMA

\section{CONTINUITY}

\section{SOME \\ REMARKS}

IN-FORMATION

AND

THE

FABRICATION
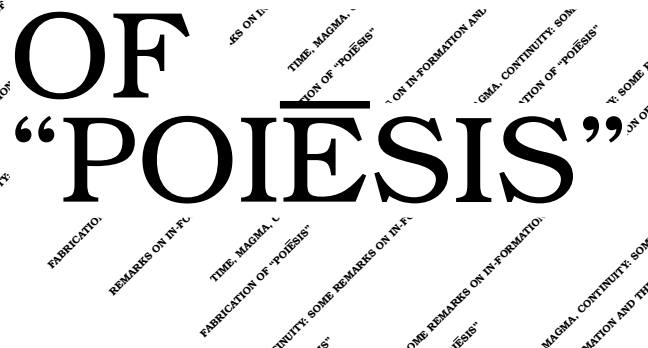

Christoph Lischka
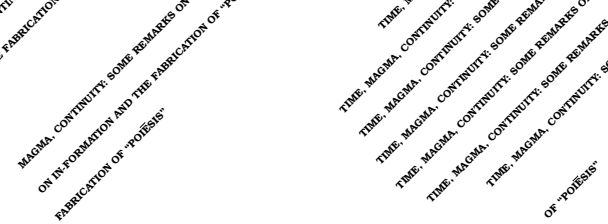
The Fabrication of Life is probably one of the most ambitious, but at the same time one of the most controversial research fields within so-called converging technologies. ${ }^{1}$ Whether Synthetic Biology ${ }^{2}$, Artificial Life ${ }^{3}$, or Bio Art ${ }^{4}$ - all these fields are concerned either way with the manipulation or synthesis of living beings.

It is well known, that organisms can be manipulated to some extent by altering their gene expression and this sort of research is already placed under quite heavy, political control. What is still less well known, and long since lurking behind the scenes, is artificial abiogenesis - the synthesis of life "from scratch". This type of research - strangely enough - is done almost completely unnoticed by the general public. Bio Art, on the other hand, seldom bothers with all those technologies - it just "applies" them, from a more or less critical stance.

A crucial question which soon comes to mind, though, is: what could "fabrication of life" actually mean? Of course - the less precise the definitions, the easier we could describe beings as "alive". Thus, for the following, we assume that a "living being" is a "being-for-itself" ("subject"), that it is a being with its own proper world ("Umwelt / Innenwelt"), and that "life" is creative: that there is an emergence of "otherness". This very process of emergence of something new - other - will be called "poiesis" or "in-formation" in the following. And we mean explicitly "fabrication": bringing life into existence (what has been referred to already as artificial abiogenesis). We do not talk about "manipulation".

Is such a project imaginable? Can we expect to become "life engineers" in the near future, building "Living Machines"?

A lot of people think it is. Some of the most advanced projects in this respect are those which are collected under the umbrella of the 6th Framework Program of the European Union, as there are SynthCells, PACE, Uniroma3, and Protocell Assembly for instance. All these projects are focussing on single cell organisms which, as general "building blocks" of more complex multicellular organisms, should, nonetheless, fit the aforementioned definitional "requirements".

Now, in order to discuss the problem of "life engineering" we want to exclude from the very beginning any framework which relies on some sort of "vital force" or a specific "bio-substance"; we don't want to introduce any

1 Bainbridge/Roco 2006; Roco/Bainbridge 2004

$2 \quad$ ETC Group 2007

3 Bedau passim

4 Kac 2006

5 Hasslacher/Tilden 1995 
type of transcendental dualism either, e.g. phenomenology. We want to stay as close as possible to contemporary scientific approaches. Having said that, however, we don't think that mainstream reductionism works. Reductionism's underlying assumption is that living beings are machinic (in the sense of a mechanism). This assumption is a consequence of a much deeper reductionist belief which is that biology has to be anchored within contemporary physics; and - usually - physics is here confined to Newtonian physics. This reductionism is not feasible for at least two reasons: first of all, sciences cannot treat "creativity" appropriately, and secondly, sciences cannot deal properly with subjectivity. As it will turn out, the ultimate cause for this failure is a profound inability to theorise time. In physics, there seems to be no notion of time at all. ${ }^{6}$ And insofar as the "sciences" in general are desperately fixated on physics, they are suffering from the same problem, too. The humanities, on the other hand, are unable to bring their concepts of "subjective time" (and/or "social-historical time") into any consistent contact with those "sciences". As a result, the dialogue between sciences and humanities either stops, or creates amusingly bizarre "discourses" - most often, for example, in the neurosciences with its perennially recurring debates about the problem of, e.g., freewill. ${ }^{7}$

One could thus get the impression that this very problem of fabrication of life indicates a paradox - it would be simply impassable. Even within biology, doubts exist about whether this reductionist strategy will work. Robert Rosen, e.g., argues, that physics "is inherently inadequate to accommodate the phenomena at the heart of biology. No amount of sophistication within these limitations can compensate for the limitations themselves." 8 In particular, since Newtonian-style physics produces analytic knowledge (i.e. knowledge, how a system works), and this knowledge does "not entail how it is created", ${ }^{9}$ the problem of fabrication simply cannot be solved.

Stuart Kauffman also considers the possibility, that contemporary physics has to be changed to become appropriate for biology. His main concern is that for principle reasons we "cannot finitely pre-state the configuration space of a biosphere." ${ }^{10}$ What he is speculating about is "glimmers ... of something like a fourth law, a tendency for self-constructing biospheres to enlarge ... the dimensionality of their adjacent possible." ${ }^{11}$ We can't go into the details

\section{Barbour 1999}

7 There is no doubt that the life sciences can produce results of some (limited) use, despite their dubious conceptual and methodological premises, e.g. in medicine with its often spectacular progress in diagnostic and therapeutic technologies, particularly in the neurosciences, see Hagner 2006.

8 Rosen 2000, p. 256

9 Rosen 2000, p. 258

10 Kauffman 2000, p. 135

11 Kauffman 2000, p. 244 
here, but we just mention in passing that Kauffman expects a "new physics" - which eventually respects biological phenomena - to be a physics carrying on the ambitious efforts tackling the challenges of quantum gravity. ${ }^{12}$

Both Rosen and Kauffman, in a way, suggest that we have to change the underlying conceptual framework of mainstream reductionism, and, most important, have to explore alternate ontologies, the main emphasis of which is on being-for-itself, creativity, and "becoming". We can go even further. Why should we conceptualise nature in terms of a "state space", introducing a "mind-body-problem" - just to "reduce" it to "materialism" or "idealism"? And why should we continue rendering the "ego" as a spectator of an external "world" - mirroring predictable trajectories and desperately trying to stay the course? There is no compelling reason at all to believe in these somewhat accidentally sedimented clichés, acting - at the best - as a common-sense "doxa".

We should be aware, though, that taking non-reductionist frameworks into consideration often means to be accused of "vitalism", "speculative thinking", et cetera - as we already mentioned above. Authors like Spinoza, Nietzsche, and Bergson are usually condemned as belonging to the "bastard line of philosophers" (Deleuze), and there is rarely a chance to engage mainstream "scientists" in a profound debate. ${ }^{13}$ An exception might be A. N. Whitehead, whose "process philosophy" happens to be discussed as an alternate ontological framework for quantum physics. ${ }^{14}$ But Whitehead is still seen as quite an esoteric thinker.

Among the (maybe) less suspicious philosophical authors who are deeply concerned with the problem of "creativity" Castoriadis comes to mind. A reading of Castoriadis seems to be rewarding from at least two points of view. As is well-known, ${ }^{15}$ he advocates the crucial role of "radical imagination" in human subjectivity. And, additionally, he develops an ontology of the "magma", which - as will hopefully be shown in this paper - allows a rethinking of "creativity" in such a way that it sheds a new, interesting light on "fabrication of life".

12 Kauffman 2000, p. 243ff

13 See the pubertal and amazingly ignorant "discussion" by Sokal/Bricmont 1998.

14 See, for instance, Hättich 2004.

15 Castoriadis 1986, 1997, 1998, 2007; Curtis 1997 
Castoriadis' philosophy, and especially his ontology, remained unfinished. It always had a strong momentum, most notably in his late writings, ${ }^{16}$ which, as I would suggest, can even be read as constituting first "building blocks" for a proprietary process philosophy.

From its very beginning his philosophy is centreed around the concept of autonomy. Autonomy is the result of a process of self-constitution or selfcreation, both on the individual and the social-historical level. We will only briefly touch on this topic here, although it opens many opportunities for criticising current approaches in neuro-, brain and cognitive sciences, first and foremost in neuropsychoanalysis.

The genealogy of autonomy is driven by imagination, "radical" as well as social-historical. Imagination as radical turns out to be the "differentia specifica" of human beings, compared with animals in general, which exhibit imagination in a functional organic context only. Humans, in contrast, have their imaginative capacities detached from any functionalisation - imagination becomes free floating: radical. During individuation - a process of psychical "sense- or meaning-making" - radical imagination evolves into both an "interior" (psychical) and an "exterior" (social-historical) equilibrium of representational pleasure, implying a compossible coupling with the underlying organic functions as well as a proper embedding into social-historical imagery. This "individuation" might fail - in the worst case resulting in psychosis. ${ }^{17}$

Thus, subjectivity of humans is anything but a fixed, rationally behaving "agency"; this might be the case, according to Castoriadis, with animals and their "hard-wired" (yet still representational!) pleasure, entwined with organic functionalities. Whereas with human beings, it's just the opposite: subjectivity is the felicitous result of an emergent creative process of radical imagination, susceptible to failure, but also open to revolutionise the world by creating "other" imageries.

The first lesson we can learn from Castoriadis, therefore, concerns the processual character of subjectivity, thwarting the mainstream caricature of rational agency. If at all, the latter turns out to be a (cynical) zoomorphism, turning the creative capacities of human imagination into pre-determined sensor-actor-circuits, receptible for computational or dynamicist models. And Castoriadis even gives us arguments against a naive adaption of the Freudian project. Whereas the latter confines psychoanalysis to the private context of the doctor's couch, Castoriadis emphasises the role of the socialhistorical mediation of imagination. That which happens at the border of

16 Adams 2003

17 Castoriadis 1997 a 
"Unconscious" and "Consciousness" is not just the "personal" fluctuation of imageries; both its genealogy and its actual virulence are deeply entangled with the exterior, social-historical. The subject is always "a bastard construct, combining in various proportions elements of the psyche, of the social-historically instituted understanding and reason, and of the self-reflecting activity of the social individual at a certain stage of history."18

As mentioned already, Castoriadis' philosophy needs to be thought of "in terms of a shift from a regional ontology of the social-historical towards a transregional ontology of physis", as Suzi Adams puts it. ${ }^{19}$ What becomes the main concern throughout Castoriadis' later writings is the logical as well as ontological difference between determinacy and indeterminacy. In order to unfold the complex interplay of both these "dimensions" of being, we first have to become aware of the stratified character of his "transregional" ontology. Physis subsists as a dynamic multiplicity of (strata of) being, which "is an irreducible, primary datum." ${ }^{20}$ As such multiplicity "formally entails unity". ${ }^{21}$ Without unity, multiplicity would cease to be multiplicity, and would become an "in itself dispersed and disconnected Infrachaos". ${ }^{22}$ Now, there are actually two ways how multiplicity exists - as difference, and as otherness. This distinction belongs definitely to the core of Castoriadis' conceptual apparatus, and it is immediately entangled with determinacy and indeterminacy, respectively.

Let us start with an example: a square is different from a rectangle, but Kafka's "The Castle" is not different from the Rolling Stone's "Satisfaction" they are other. According to Castoriadis, two forms are different "if there is a set of determinate transformations ('laws') allowing the deduction or production of this form." ${ }^{23}$ "Determination" has to be taken in its most general reading, as being an identitary element of an ensemble - i.e. set-theoretically. Because of its overarching importance Castoriadis coined a new term for this "ensemblistic-identitary" logic: ensidic. This logic is "hard-wired" into our language; it is the basis for all mathematical constructions, and is the underlying logic of our sciences. ${ }^{24}$ Theorising along ensidic lines results in a construction of hierarchies of sets, equipped with relations and rules of deduction. Ensidic thinking "spatialises" multiplicities insofar as it constructs unities by identifying elements and collecting them as an ensemble. It neglects any intrinsic

\footnotetext{
18 Castoriadis 1997b, p. 377

19 Adams 2003, p. 106

20 Castoriadis 1997 b, p. 400

21 Castoriadis 1997b, p. 399

22 Castoriadis 1997b, p. 399

23 Castoriadis 1997b, p. 392 (with the author's emphasis)

24 Castoriadis 1997 c, p. 295
} 
characteristics and figures these elements simultaneously, yet coexisting as different ones just by external organisation.

There is no depreciation of ensidic descriptions (in contrast, e.g. with Heidegger's "Vorhandenheit"), rather the opposite is the case - they constitute the dominant form of our world representation. Because the "first natural stratum" itself allows for ensidic constructions, social-historical imagination, individual humans are capable of instituting viable representations of their respective worlds. The problem arises if we exclusively turn our attention to ensidic narratives, reducing the multiplicity of being to simply a differential one. A world made up only out of differences wouldn't change anymore, and nothing new would happen. All is determined, only differences exist: the repetition of the same. But the "new is not the unforeseeable, unpredictable, nor the undetermined." ${ }^{25}$ The (unpredictable) next number in roulette, for instance, still remains "the trivial repetition of a form", as does the undetermined, "sheer repetition of a given form" in quantum mechanics. ${ }^{26}$ The "new" requires the indeterminate, the magma, which allows for the emergence of new determinations, of new laws; this "is the meaning of form - eidos." 27

How does this in-formation (poiesis), the emergence of the "other", arise? We already mentioned the second way of how multiplicities exist: as otherness. Otherness cannot occur out of ensidic space. Ensidic space only knows of differences, forms, where each form can be derived or produced from other forms, by determinate laws. No new forms emerge. Hence, we might consider time. New forms emerge in time, don't they? - It depends.

Castoriadis' extensive analyses first show us why "creation", the emergence of "otherness", can't be described by physics and related sciences. The reason is simple: they see "time" exclusively as ensidic time - social identitary time, which leans on the ensidic dimension of the first natural stratum. ${ }^{28}$ This, in turn, implies the spatialisation of time in the sciences, and results in the reduction of temporal multiplicities to differential ones.

Therefore, time in general does not really help. We have to take into account the magmatic dimension of time. The emergence of forms (in-formation) is the ultimate character of time. The "before" and "after", the irreversibility of poietic time, is "given through the scansion of creation and destruction." 29 Poietic time forces a self-deployment of new forms in ensidic space and time as receptacles of the first natural stratum, where they become organised through subjective - both social-historical and individual - constructions. Forms as forms are not caused by something, in the sense of determinate necessary and

25 Castoriadis 1997 b, p. 392

26 Castoriadis 1997b, p. 392

27 Castoriadis 1997b, p. 392

28 Castoriadis 1997b, p. 387

29 Castoriadis 1997b, p. 397 
sufficient conditions; they emerge - given appropriate (innumerable, but only necessary) conditions. "The conditions allow the emergence of the form - but the converse is meaningless." ${ }^{30}$ In-formation is ex nihilo, which does not mean in nihilo, or cum nihilo. The magma allows for in-formation, but it cannot be exhausted; the ensidic is indefinitely "dense" in the magmatic.

3.

As previously suggested, we could start reading the late Castoriadis as process philosophy. In particular, if we focus on his text "Time and Creation", ${ }^{31}$ we will detect a clear prominence of time over space - the two "receptacles". When Castoriadis asks whether there is "a possibility for an essential distinction between time and space" ${ }^{32}$, in the end he gives priority to time: without time there would be "no thing (nothing)" 33 . Nonetheless, time and space are intimately entangled for multiplicity exists both as difference and as otherness, and "otherness entails difference" ${ }^{34}$. This, in turn, implies that every form - in order to be - has to be "identical to itself", it has to persist for a while, qua pure repetition in ensidic time - differing with itself "only by being placed in a different (identitary) time" 35 . Thus, every form has "necessarily an ensidic dimension". And Castoriadis' ontology establishes a clear priority: being is time. "The fullness of being is given - that is, simply is - only in and through the emergence of otherness which is solidary with time." 36

Finally, then, in-formation (or poiesis) - the "surging forth" of otherness as characteristic for being - forces the fragmentation and stratification of being. Qua self-deployment, being forces the proliferation of otherness, dispersing new forms both in poietic and ensidic space and time. As poietic receptacles, space and time ensure alterity; as ensidic, they establish the Being of being at all. Thus, the emergence of otherness, in-formation, does not contradict determinism; it rather contradicts "the paradoxical, if not absurd, idea of a homogeneous universal determinism that could reduce level or strata of being (and their corresponding laws) to a single ultimate and elementary level." ${ }^{37}$ Creation ruptures the smoothness and continuity of being, it foils reductionism.

30 Castoriadis 1997b, p. 397

31 Castoriadis 1997b

32 Castoriadis 1997b, p. 397

33 Castoriadis 1997b, p. 399

34 Castoriadis 1997b, p. 400

35 Castoriadis 1997b, p. 400

36 Castoriadis 1997b, p. 401

37 Castoriadis 1997b, p. 393 
It might be worthwhile looking at contemporary sciences with respect to their concept of emergence. Emergence - as is well-known - is currently often seen as a new "weapon" in the hand of reductionists. Teeth-gnashing, physicists are beginning to accept that there are "levels" or "strata" of being - probably forced by their very problems with thermodynamics and quantum mechanics. Yet ideally, these strata are communicated as hierarchically ordered, with a one-way determinism from the bottom up. For example, the (phenomenological) variables of thermodynamics (like "temperature"), which constitute a "higher level" of description, are reduced to the movement of molecules - a "lower" descriptive level, more "fundamental", and thus explaining the macroscopic phenomenon. This example illustrates exactly what Castoriadis complains of as "homogeneous universal determinism". Admittedly, more advanced conceptualisations of "emergence" are "emerging"38 - it might be promising to relate them with Castoriadis' "Logic of Magmas"39.

Castoriadis tried several times to elucidate his concept of "magma", the most detailed attempt probably is his paper "The Logic of Magmas and the Question of Autonomy". ${ }^{40}$ I don't want to comment on this paper here explicitly; rather I would like to emphasise his reference to quantum mechanics. In this paper he mentions Mugur-Schächter, a physicist, talking about her reflections during theory-building in quantum mechanics. In the end, she finds herself within a "semantic mud", and "it is only here, in this mud, and when we force our gaze to make out the moving forms, that we can perceive the contrasts between what is not done and what is partially done and thus initiate something anew." ${ }^{41}$ Mugur-Schächter alludes to their problems with the vanished subject-object separation in quantum physics, and the difficulties of handling these problems semiotically.

This reminds us - and that is probably the reason why Castoriadis quotes her - of his account of subjectivity. Again, in his paper "Time and Creation" ${ }^{2}$, Castoriadis develops his concept of space and time, and how they relate to subject and object. Just remember: the world is socially constituted (via imaginary institutions), and it "appears as the deployment of two receptacles, social space and social time, filled with objects organized according to relations, etc., and vested with meaning." ${ }^{43}$ Receptacles appear to a subject. But they lean on the first natural stratum with "respect to [their] ensidic dimension" ${ }^{4}$. Every living being (being-for-itself) "know(s) ... at least some-

\footnotetext{
38 Bishop/Atmanspacher 2006

39 Castoriadis $1997 \mathrm{c}$

40 Castoriadis $1997 \mathrm{c}$

41 Castoriadis 1997c, p. 303

42 Castoriadis 1997b

43 Castoriadis 1997b, p. 386

44 Castoriadis 1997b, p. 387
} 
thing of the world." This implies that the world "is knowable"; but it has to be "constructible" as well. ${ }^{45}$ The world "must contain the ... equivalent of an identitary dimension." 46 We don't have a chasm between "subject" and "object", however - as is still the case in mainstream thinking, as long as it relies on Newtonian physics. It is rather a chiasmus (Merleau-Ponty) of subject and object; their respective parts of these constructions cannot be disentangled. Yet, our effort to separate them is not "... meaningless, on the contrary; but it is bound to be interminable." ${ }^{47}$

We might understand better now, why Castoriadis was seduced by quantum physics: the latter turns out to be the reference for our interaction with the world. The fundamental interactional pattern between subject and object is quantum mechanical, and not Newtonian. Our world is a world of "Zing!" 48 Jean-Yves Girard is one of the first logicians who strongly emphasises that we have to stop imposing an ensidic logic (to use Castoriadis' term) in theorising about nature (as so-called quantum logic does); rather we should take noncommutativity seriously, and create a new logic which picks up the insights of quantum physics, and develop a logic along the lines of the principal imbrication of observer and system. ${ }^{49}$ One of the most fascinating results of this approach is the relativisation of set theory - it simply becomes "local", a subjective "viewpoint" of an observer. If we recall Castoriadis' attempt to describe the interplay between the magmatic and the ensidic, the emergence of "new" determinations during the interaction of being-for-itself and its proper world - maybe with Girard's "Geometry of Interaction" 50 we have found a promising departure for the conceptualisation of the "everywhere dense" ensidic within the magmatic - in-formation.

\section{4.}

If we now turn back to our very question - How is fabrication of life possible? - we should first stress the fact that Castoriadis' philosophy/ontology in general seems to be an attractive, competitive framework for theorising about life and technology - from single cell organisms up to the attempts of the neurosciences to model human behaviour. Unlike Heidegger, or other philosophers in the phenomenological tradition - with their often exposed technophobic attitude - , Castoriadis has a brilliant background both in mathemat-

45 Castoriadis 1997b, p. 387

46 Castoriadis 1997 b, p. 389

47 Castoriadis 1997 b, p. 389

48 Fuchs 2002

49 Girard 2007a, 2007b

50 Girard 2006, 2007b 
ics and in the sciences, which often allows him to avoid bizarre conclusions and misinterpretations.

Secondly, there exists an explicit examination of Varela's approaches to biology, ${ }^{51}$ focussing mainly on the concepts of autonomy and being-foritself. The crucial question is whether we can think "the living being as a fully ensemblistic-identitary automaton"; an automaton, that "has in itself the principles of its generation and corruption as well as of its alteration."52 Castoriadis simply does not know. Yet he doubts that it could be possible, for the following reasons. Even if it would be possible to create a complete ensidic description (and construction) of a dog - including an isomorphism between the dog's own significations and constructions within its proper world, and the external ensidic description of the automaton - even then, this "artificial" dog wouldn't be "new", it would be just a replica of an already existing system, whether this is "in the head" of the engineer, or a natural "template". And this, according to Castoriadis, seems to be implicit in ensidic logic: we would never have the reason, nor the criterion for fabricating the dog, if "the dog did not already exist." 53

We could go beyond Castoriadis (and Varela), and might consider implicit fabrication. This would generally imply the ensidic determination of a "parameter space" - whether discrete or continuous. It would need a (determinate) "quality measure" as well as a (determinate) "procedure", driving the system through the parameter space. Eventually, a being-for-itself might "evolve". Two cases can be considered. First, the system "emerged", it worked as intended, and nothing "extra" happened. This wouldn't change the scenario at all, the same arguments as before would still be valid. In the second case, though, we could imagine that this being-for-itself does not match the "target requirements" (or perhaps there were no requirements in the first place), but beyond exhibiting its proper world, it would also exploit the magmatic dimension of the world during the construction of this very world - due to the (potential) exploitation of this magmatic dimension during "evolution". Yet, what we have got now is a completely different concept of fabrication. There is almost no control anymore - neither of the "result", nor of the schedule of the process itself. And this leads us to the last issue.

Populating the world with beings-for-itself is just a special, though very prominent, case of the emergence of otherness - poiesis. Thus, the question of the fabrication of life entails the question of the fabrication of poiesis, and as we have just seen, this implies a change of the concept of fabrication. With fabrication we have actually two choices: we can lean on the ensidic, or we

51 Castoriadis 1997d, pp. 337-339, 1997c, pp. 308-310

52 Castoriadis 1997c, p. 309

53 Castoriadis 1997 c, p. 310 
adhere to the magmatic dimension of being. In the first case we start creating ensidic constraints, determining "primitives", production rules, and try to minimise alterity - producing forms by repetition and difference. We try to occupy the magmatic dimension of the world, so to speak, and substitute it by our own radical and social-historical imagination, eventually blowing out the poiesis of the world. We should be honest: as cowardly as we are (as a species), this world would end up in an eternal return of the ever same - boredom.

The second choice we have would be to exploit the poiesis of the world. We would "listen" to the world - intensifying the emergence of otherness, enjoying the fecundity, and subversively reinforcing the overwhelming proliferation of different strata of being, disrupting continuity and thereby undermining the totalitarian pretense of the ensidic.

Fabrication of poiesis, then, means keeping open the surging forth of physis: alloiōsis. It works out to be simply waiting for the right moment, the kairos - with Gelassenheit.

If you want - an ethics of in-formation. 


\section{References}

6th Framework Program of the European Union (2008). Online available: <http: / cordis. europa.eu/fp6/> (last access: June 2008).

Adams, Suzi (2003): „Castoriadis' Shift Towards Physis«. Thesis Eleven 74, 105-112.

Bainbridge, William Sims /Roco, Mihail C. (Eds.) (2006): Managing Nano-Bio-Info-Cogno Innovations: Converging Technologies in Society, New York: Springer. Online available: <http://www.wtec.org/ConvergingTechnologies/3/NBIC3_report.pdf> (last access: June 2008).

Barbour, Julian B. (1999): The End of Time, New York: Oxford University Press.

Bedau, Mark A. (Ed.) (1993ff.): Artificial Life (Journal), Cambridge: MIT Press.

Bishop, Robert C./Atmanspacher, Harald (2006): »Contextual Emergence in the Description of Properties«. Foundations of Physics 36(12), 1753-1777.

Castoriadis, Cornelius (1986): Crossroads in the Labyrinth, Cambridge: The MIT Press.

Castoriadis, Cornelius (1997): World in Fragments: Writings on Politics, Society, Psychoanalysis, and the Imagination, Stanford: Stanford University Press.

Castoriadis, Cornelius (1997a):»The Construction of the World in Psychosis« In: Cornelius Castoriadis, World in Fragments: Writings on Politics, Society, Psychoanalysis, and the Imagination, Stanford: Stanford University Press, 196-210.

Castoriadis, Cornelius (1997b): „Time and Creation«. In: Cornelius Castoriadis, World in Fragments: Writings on Politics, Society, Psychoanalysis, and the Imagination, Stanford: Stanford University Press, 374-401.

Castoriadis, Cornelius (1997c): »The Logic of Magmas and the Question of Autonomy«. In: David Curtis (Ed.), The Castoriadis Reader, Oxford: Wiley-Blackwell, 290-318.

Castoriadis, Cornelius (1997d): »Phusis and Autonomy«. In: Cornelius Castoriadis (1997): World in Fragments: Writings on Politics, Society, Psychoanalysis, and the Imagination, Stanford: Stanford University Press, 331-341.

Castoriadis, Cornelius (1998): The Imaginary Institution of Society, Cambridge: MIT Press.

Castoriadis, Cornelius (2007): Figures of the Thinkable, Stanford: Stanford University Press.

Curtis, David (Ed.) (1997): The Castoriadis Reader, Oxford: Wiley-Blackwell.

ETC Group (2007): Extreme Genetic Engineering: An Introduction to Synthetic Biology. Online available: <http:/ /www.etcgroup.org/upload/publication/602/01/synbioreportweb.pdf> (last access: June 2008).

Fuchs, Christopher A. (2002): Quantum Mechanics as Quantum Information (and only a little more). Online available: <http://lanl.arxiv.org/abs/quant-ph/0205039vl> (last access: June 2008).

Girard, Jean-Yves (2006): Le Point Aveugle: Tome 1. Cours De Logique, Vers La Perfection, Hermann. Online available (english): <http://iml.univ-mrs.fr/ girard/ coursang/coursang0.pdf.gz> (last access: June 2008). 
Girard, Jean-Yves (2007a): »Truth, modality and intersubjectivity«. Online available: <http: / /iml.univ-mrs.fr/ girard/truth.pdf> (last access: June 2008).

Girard, Jean-Yves (2007b): Le Point Aveugle: Tome 2. Cours De Logique, Vers Limperfection, Hermann. Online available (english): <http://iml.univ-mrs. $\mathrm{fr}$ / girard/coursang/coursang0.pdf.gz> (last access: June 2008).

Hagner, Michael (2006): Der Geist bei der Arbeit: Historische Untersuchungen zur Hirnforschung, Göttingen: Wallstein.

Hasslacher, Brosl/Tilden, Mark W. (1995): »Living Machines«. Robotics and Autonomous Systems 15(1-2),143-169.

Hättich, Frank (2004): Quantum Processes. A Whiteheadian Interpretation of Guantum Field Theory, Münster: agenda.

Kac, Eduardo (2006): Signs of Life: Bio Art and Beyond, Cambridge: MIT Press.

Kauffman, Stuart A. (2002): Investigations, New York: Oxford University Press.

PACE. Online available: <http://www.istpace.org/> (last access: June 2008).

Protocell Assembly. Online available: <http://protocells.lanl.gov/> (last access: June 2008).

Roco, Mihail C./Bainbridge, William Sims (Eds.) (2004): Converging Technologies for Improving Human Performance: Nanotechnology, Biotechnology, Information Technology and Cognitive Science. Berlin/New York: Springer. Online available: <http: / /www.wtec.org/ConvergingTechnologies/1/NBIC_report.pdf> (last access: June 2008).

Rosen, Robert (1991): Life Itself, New York: Columbia University Press.

Rosen, Robert (1999): Essays on Life Itself, New York: Columbia University Press.

Sokal, Alan/Bricmont, Jean (1998): Fashionable Nonsense: Postmodern Intellectuals' Abuse of Science, New York: Picador.

SynthCells. Online available: <http:/ / www.synthcells.org/> (last access: June 2008).

Uniroma3. Online available: <http://www.plluisi.org/grl_res_index.html> (last access: June 2008). 


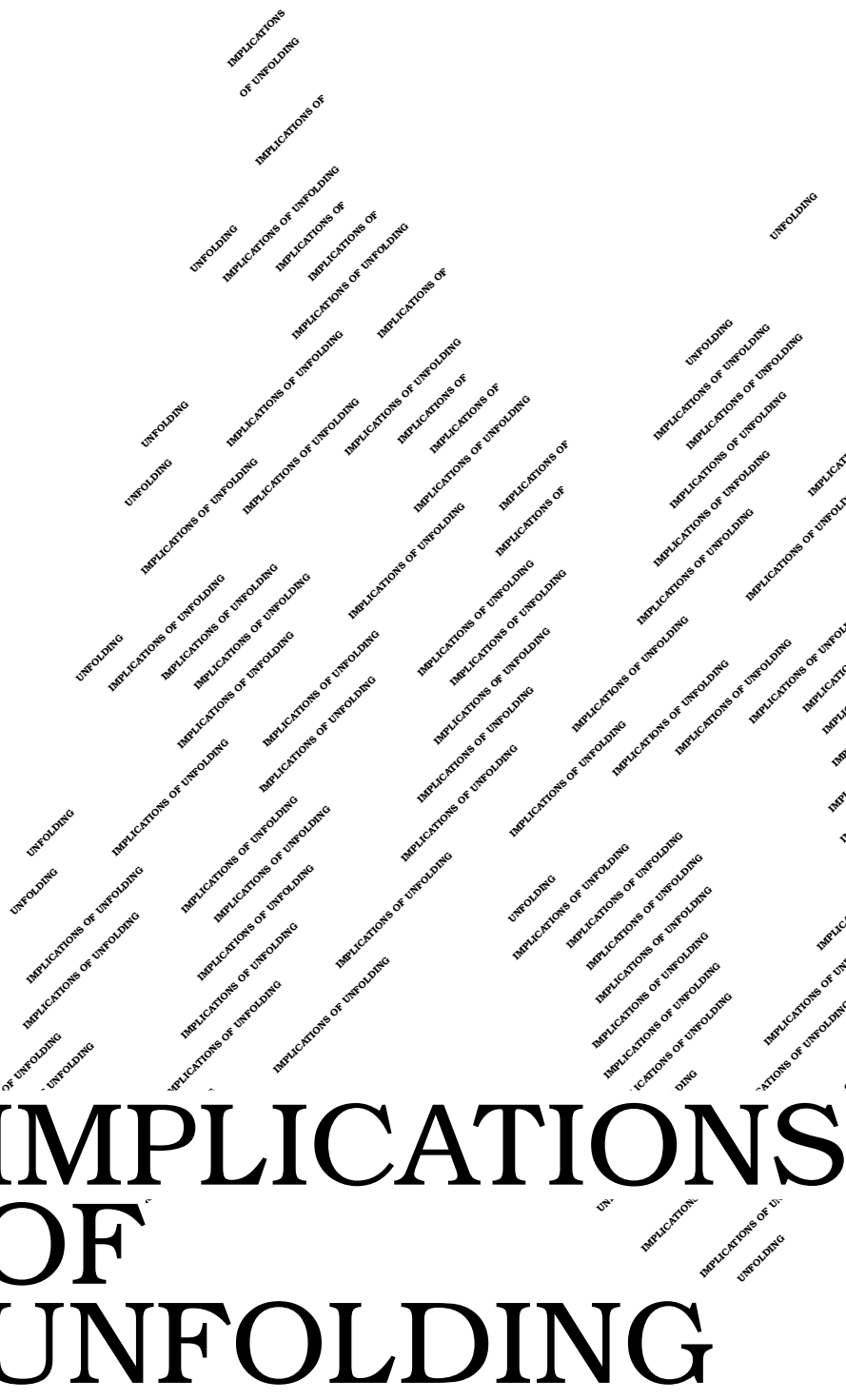

Juilian Rohrhuber

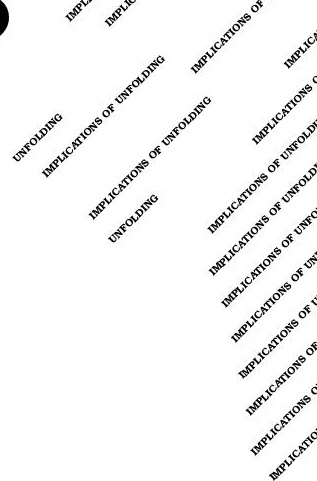


In spring 2002, the scattered fragments of a meteorite entered the earth's atmosphere and plunged to the ground close to the 19th century Neuschwanstein Castle. The impact did not cause any damage, but was noticed by local observers and its trace recorded by astronomical camera networks. ${ }^{1}$ After a reconstruction of the trajectory from the photographic data and some systematic search in the field, two pieces were retrieved on German territory. Some time later, following a corrected model of the expected shape, a third and largest fragment was found by a German physicist across the nearby Austrian border. Since these meteorites (of the enstatite chondrite type) are well recorded and of relatively high value, their material presence immediately caused a conflict between potentially rightful owners, such as the mayor of the small town of Reutte (by proxy), and the finder. The court case turned out to be intractable - the most heterogeneous categories of law had to be taken into consideration; after all, the laws that govern the interaction between heavenly bodies do not regulate the accumulation of wealth.

Seen from a distance, all that had happened was a minor extension to the planetary material. However, according to extant law, the situation could not be easily decided. Is a meteorite like snow (which 'falls' under the responsibility and property of the landlord), is it like apples from neighbouring premises, or like flotsam (which is regulated by specific laws)? Is it to be considered a natural monument? A report by the Bavarian state lawyer Kristine Faust discussed these issues circumspectly; she clarified that a meteorite is not material fallen from neighbouring premises, and that the ground it has fallen on has not produced it either. ${ }^{2}$ Only something that is lost, can be found, and as the state had not acquired the meteorite in the moment of impact, it was not lost property either: despite the fact that gravity may be enough to juridically bind a thing to its premises, the meteorite was still light enough to be easily removed without the application of "disproportional effort". Yet with the first fragment, Faust came to the conclusion that the case was analogous to the discovery of hidden treasure, a solution that led to simply cutting the stone in two halves of equal weight, one for the state, one for the discoverers, who divided their half and sold the fragments. In the second case, the claim by the Austrian town was delivered a rejection, culminating in the statement "there is no earthly right to heavenly goods", and ownership was granted to the finder. ${ }^{3}$

1 The fish-eye camera propeller of the European Fireball Network scans a complete night sky every night. The photographic observation was published in Spurný et al. 2003, pp. 151-153.

2 Faust 2003, pp. 28-31

3 It remained unclear whether the then rightful owners had to pay income tax for their new possession.

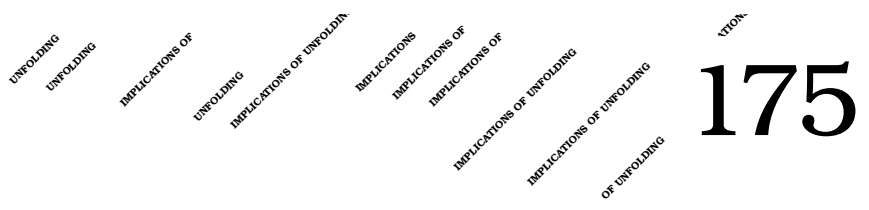


Affiliations between things and living beings take the most diverse forms; this theme could hardly be more quotidian - everyday life is occupied with houses, tools, vehicles, but also with the material reality of weather and gravity, and trying to separate environment from inhabitant may at any moment turn out to become a knotty issue. Also, nothing stops us from extending the realm of things to languages, signs and symbols, which can forcefully turn out to condition an umwelt, just as they can assume the place of implements. So when it comes to the origin of actions and intentions, this context dependency makes it necessary to consider interactivity as fundamental to any investigation. There remains a certain dichotomy here though: while objects, structures, rules, or laws depend on a particular timelessness, movements, processes, interventions are almost exclusively temporal.

As the observation of scientific and artistic practices shows, it would be a mistake to locate the origin of attributes like intentionality or initiative in the human mind alone. Social, material, structural circumstances force decisions, just as they are subject to modification and investigation. It is possible to avoid a foundational choice between a social constructivist and a realist view by a different account of objectivity. This objectivity is the result of interaction. Such "situated knowledges", as Donna Haraway writes, "require that the object of knowledge be pictured as an actor and agent, not as a screen or a ground or a resource, never finally as slave to the master that closes off the dialectic in his unique agency and his authorship of 'objective' knowledge." 4 In this context, it is significant that many theories seem to have shifted agency toward objects instead of looking for it in processes. In Alfred Gell's anthropology of art, for instance, the main agent is the material art object. ${ }^{5}$ For Donna Haraway, and also for Michel Callon and Bruno Latour it is the various hybrid, yet material coalescences that defy categorisation as either natural or social, animate or inanimate; they take the shape of collectives, 'agencements', which, despite their multiple forms, tend to crystallise in matter. ${ }^{6}$ Hans-Jörg Rheinberger proposes an intermediate form between concept and object, the epistemic thing. ${ }^{7}$

Other than simply resisting common preconceptions, there are also good reasons for applying the notion of autonomy to material objects rather than to processes merely because the latter appear closer to 'being alive'. Firstly, objects imply specific actions and inherent necessities - they can be

\footnotetext{
$4 \quad$ Haraway 1988

5 Gell 1998

6 Haraway 1988; Latour 1993; referring to Donna Haraway, Callon writes, "These agencies, like Hobbes' Leviathan, are made up of human bodies but also of prostheses, tools, equipment, technical devices, algorithms, etc. The notion of a cyborg aptly describes these agencements." (Callon 2005)

7 Rheinberger 1997
} 
described in terms of constraints and resistance, both physical and social. In a sense, things implicitly encode processes. A ball in a game is a classic case. ${ }^{8}$ Secondly, an object occupies a place, may be attached to owners, can be passed on, and is thus able to transport action patterns between nodes in a network of relations. It may itself become a node, being equally the subject of, and subjected to, new formations. Not to mention that things can be traced, and sometimes collected. Nevertheless, according to agency theories, it would be wrong to treat objects (as well as subjects), as primary to their relationships with each other. Rather, it is the association between agent and patient that results in the resistance, the stubborn, 'objective' ignorance toward change that causes objects to exist, somewhat like the apparent stasis of an eddy or vortex in the flow of a stream. These linkages, which French sociology termed operation chains ${ }^{9}$ are transactions of potential action. ${ }^{10}$ In this capacity, they are agents with social leverage. Because they result from these chains, objects are inherently political.

The decision to put aside the essential polarity between the agency of persons and the agency of things allows us to treat the collective situation as existing logically prior to subject, action and object, and to render them conceptually indistinguishable. Deliberately creating a mode of observation with a blind spot for these distinctions causes new differences, subjects, and situations to appear. Areas of thought that tend to suffer when actions are merely considered as transmission from internal intention to external expression can be better explored by not presupposing objects to which intentions and actions of subjects can be moored. Thus, issues of intentionality in art and the intricate relation between discovery and fabrication that constitutes objectivity in the sciences cease to disturb investigation. In what way, for instance, does an artwork participate in the possibility of its own formation or condition its own becoming as it unfolds? How does a discovery turn out, after the fact, to constitute the very place it must have been part of already? In such issues, rather than a hindrance, paradox and undecidability turn out to be the driving force that opens previously inconceivable possibilities. "Experimental systems," Rheinberger writes, “[...] allow researchers to arrive at unprecedented, surprising results. In this sense, such systems are

8 For Michel Serres, the football is a good example for what he calls a 'quasi-object'. His figure ground reversal illustrates how agency and patienthood may swap places: according to this perspective, in a football game, it is not the players who control the object. Conversely, is it the ball that is the subject of circulation between stations, and the players follow after it. A quasi-object is only an object insofar as its movement binds a collective (Serres 1987; Roßler 2008).

9 The term operation chain (chaîne opératoire) was introduced by Leroi-Gourhan in 1964. It has its origin in archeology, where it had been developed out of the research on the action patterns in the fabrication of Stone Age tools, traceable from raw material, completed artifacts, and their chippings on production sites (Leroi-Gourhan 1964).

10 Schüttpelz 2008 
'more real,' if you will, than ordinary reality. The reality of epistemic things is their resistance, their resilience, their capacity, as 'jokers' of practice, to force us to abandon preconceptions and anticipations." ${ }^{11}$ In the following, I will discuss agencies that force the formation of a series of conjectures, of open hypotheses. Especially I will do so with respect to a kind of inconsistent hybrid between representation and unfolding, which may be called a 'model'. A detour into a specific praxis of computer programming, interactive programming, will provide evidence for a specific kind of interactivity typical for experimental systems; it can be traced back to an agency in the formal. Here, interactivity will turn out to imply a rather simple, temporal paradox; instead of looking for it in an immediate 'presence' of coupling, this observation will help to show how the unfolding of an investigation implies interactivity between its own history and future.

\section{Program, model, trap}

Taking operation chains as causes underlying the formation of objects does not imply that these objects explicitly represent actions. Art objects, for instance, may be cunningly prepared in a way to cause a certain impression, and to inhibit others; they may function to impress the audience, or a patron; tools, or other objects usually imply certain actions, but are not self-explanatory; such things are a part, or a trace of an operation chain, but do not give access to the chain itself. Nonetheless, there are also many cases in which operativeness is combined with its description e.g. calenders, maps, plans, recipes, algorithms. A program is one example of a thing with such a double nature: it explicitly provides a plan together with a method to actualise it. The text of a program represents two processes at the same time: in the context of a given computer language, it activates a computational process, which may (or may not) produce results. Second, and this is what is supposed to qualify a 'good' program, it causes a human reader to understand, in one way or another, this process. A program can be regarded as 'operative writing"12, as an assemblage of a possible process and its description.

As in any language though, one should not expect a transparent translation from formula to meaning, or a complete reflectiveness between process and description. The hybrid assemblage of operation-representation is necessarily incoherent. An example of a process that produces a description may illustrate this; since we have to describe this process of description-making, we can think of the 'simplest' case as a description which describes nothing but its own making. Such programs whose output coincides precisely with

11 Rheinberger 1997, p. 246

12 Krämer 1993 
their own source code (quines) are often fairly hard to understand; in order to write a program that is a description of how to type out its own text, one usually has to construct a maze of means and ends, of 'use' and 'mention', of quoting and unquoting of quotes. This may be a clue that there are good reasons for the fact that the description of an operation chain resists superposition with the chain it produces. Here, the semantics of such a program is its code in a literal form. ${ }^{13}$ Yet from a different perspective, it describes the process of producing this very code; in other words, it is because objectand meta-language interrelate that makes a quine difficult; in less reflective programs, where means and ends are more separate, this difficulty is not so obvious.

In a formal language, the semantics of a sentence, its meaning, is called a model. Also, a model of a whole language is all that can be 'expressed' in it. The model as such is a purely mathematical concept that usually refers to an abstract domain; yet at the same time, algorithms are, in a sense, the mechanical equivalent of a part of mathematical praxis. So it is justified to ask what a given program-text really means from a formal point of view what is its model? With respect to our train of thought, three possibilities are obvious. Does it express (1) its result, which is the effect and endpoint of its execution? Does it express (2) the process that leads to this effect? This is not obvious at all. In fact, looking at the details of specification, this will always remain a slightly ambiguous issue; ${ }^{14}$ what seems like a description of a result (a domain) may take on a more operative aspect (a process) in another situation; in other words, operation chain and its effect can never entirely be disentangled. And (3), we may have to take the program literally and see in it an inscription of the programmer's thought rather than a direct description of either process or result. ${ }^{15}$ Usually, these three levels are arranged in an

13 Note that this may be any kind of representation, which need not be 'text' formatted in ASCII code. A quine in a visual programming language, for instance, would have to compute its own visual code as an image, without re-using parts of this representation.

14 This difference can be formalised in computer languages, but most languages do not do this. Even with a formalised semantics, semantics remains a matter of decision (For a thorough discussion on algorithmic equivalence, see Blass et al. 2008). Formal systems like pi-calculus explicitly encapsulate semantics into the system, which is passed around between agents. Computer semiotics, on the other hand, emphasises the process of meaning production as interactive coupling between cultural and algorithmic processes (Andersen 2003; Nake 2003).

15 Alternatives (1) and (2) correspond to two different understandings of formal computer language semantics: operational semantics refers to the computational steps of its process, whereas for denotational semantics (this term is a bit misleading) the process does not matter, as it refers only to its eventual outcome. Note that this outcome may, in turn, be a process, such as an interactive application. In most discourses, the meaning of a program for the programmer (3) is not considered separately. Nevertheless, emphasising the human reader and the communication of ideas, the concept of literate programming brought forth by Knuth (1992) and Iverson (1979) requires code to be taken more literally. Andersen (2003, p. 190) even takes formal semantics to be "the rules that we employ ourselves to read a piece of program," and the compiler a "machine execut- 
instrumental relation; code simply expresses its result, not unlike a pocket calculator. This is because when a computation is fast and leads to some sort of unchanging entity, such as a number or the data of an image, the process can be thought to implode in the blink of an eye.

The situation is a little bit different as soon as the program is to describe a process that unfolds over time. In the above case we can say that a reckoner's operational agency (and labour), calculating by hand, is replaced, and hidden, in the rules of the formal system. This is not so obvious when the program's result consists in processes, possible behaviours, actions. Here, it is easy to lose the distinction between the program as a process that leads to another process, and this latter process (which is a program too). The 'application' replaces the 'program'; possible interactions with the application are identified as the 'behaviour of the program', so that now the assemblage between plan and process is solidified in a thing, such as an interactive application, but also as an interactive installation, augmented environment, etc. In the first case it was the effectiveness of the algorithm that made it possible to neglect the ambiguity between process and product, in order to command a view on the relation between timeless formula and its immediate computational result. Here, it is the interactive computation that, by representing the behaviour of a possible agent, replaces the semantic ambiguity. On the one hand, the fact that now computation happens necessarily over time, makes it more obvious that a program is an automaton, which works exactly in so far as it has been abandoned by its programmer. The model is left behind; instead of being the originator's operational result (like a painting is the artists product), it embodies the originator's formal ghost. On the other hand, this autonomy also introduces the above double meaning between processes, where the 'making of' interactive behaviour hides in the runtime behaviour itself. The model becomes objective because it is, to a degree, independent, it is an operational proof of its own unfolding.

This is a reason why attention has been drawn to the notion of modelling as an ideological notion, both from epistemology and computer semiotics. While semiotics tends to suggest a constructivist position instead, where programmers create a reality, ${ }^{16}$ and the model of a program is a metonymical and metaphorical structure, within a materialist epistemology, programming (or formalisation in general) is taken as an experimental process with its own structural constraints and mathematical domain: "A formal system is a mathematical machine, a system for mathematical production and is placed within the process of this production." ${ }^{17}$ Objectivity is not a function of behav-

able Representation of this." To regard programs as narratives, or as discursive media has become more widespread today.

16 Noble et al. 2002

17 Badiou 2007, p. 43 
ioural resemblance of an artificial object with the natural object of enquiry, but it is to be found in the stubborn openness of formal systems. Precisely in so far as the model has been left behind, it is an agent, and its behaviour subject to discovery.

There is a well-known cultural technique, which differs from numerical calculation yet represent a type of reckoning that may help to clarify this issue, or at least may show a way into further investigation. In his 1996 article Vogel's Net, Alfred Gell gives an analysis of cultural praxis that bears many aspects of his concept of agency of artefacts which he published two years later. For Gell, animal traps are a peculiar kind of thing capable of more than it seems at first glance. Essentially, a trap is a mechanical implement of the hunter's ability to catch or to kill. Its reactivity is a model of the hunter's awareness, its mechanism is a model of cognitive competence. "It is, in fact", he writes, "an automaton or robot, whose design epitomizes the design of its maker. It is equipped with a rudimentary sensory transducer (the cord, sensitive to the animal's touch). This afferent nervous system brings information to the automaton's central processor (the trigger mechanism, a switch, the basis of all information-processing devices) which activates the efferent system [...]. This is not just a model of a person, like any doll, but a 'working' model of a person." Similar to Latour's example of the 'sleeping policeman', an object takes the place of a human in the enchainment of causes and effects. Yet, Gell notes that, at the same time, the trap is not only a model of a hunter. A trap is an altered environment - not so much as the hunter perceives it, but rather as a portrait of the animal's perceptual Umwelt. In order to catch, it is a model of an observer being caught. Gell notes that "[...] if we look at traps, we are able to see that each is not only a model of its creator, a subsidiary self in the form of an automaton, but each is also a model of its victim. This model may actually reflect the outward form of the victim [... or] the trap may, more subtly and abstractly, represent parameters of the animal's natural behaviour, which are subverted in order to entrap it. Traps are lethal parodies of the animal's umwelt." 18

As a superposition of two models of complementary observers, I think it is plausible that the agency/patienthood of a trap resembles that of an interactive program. Its mechanism is a concrete abstraction, a passe-partout of its parameters. Like a trap, a program is "a model as well as an implement." 19 Program and trap both encapsulate a hidden, objectified plan that unfolds into a scene only at the appropriate circumstances. They are situated models of a silent, absent observer which is present in the observation of an observer. The conditionality and sequentiality of an algorithm come to a halt

18 Gell 1999, p. 200

19 Gell 1999, p. 200 
when it snaps shut. But what is actually caught by a program? And who is the hunter really? Is there a moment of closure at all? The analogy could be a trap itself.

Apart from notable exceptions, the process of interaction, as it obtains between a running program and its environment, seems to have little of the sudden abduction by a mechanical implement. And when it is not meant to ensnare a potential person, but rather to give access to a new situation and unforeseen observations, it cannot be simply a portrait of either cognitive hunter or cognitive prey. Rather it appears to shift between an instrumental aspect, where computation simulates physical processes or enables communication, and a more oblique situation, in which a given causality is disturbed, where it is not clear what actions find continuation and which percepts are consequences of the local logic. One aspect is easily forgotten though, when looking at real-time interaction in art, or also in scientific simulations. Because, unlike in the early days of computing, human computer interaction today involves mostly the relation between algorithmic processes and users, the activity of constructing a program in the first place is taken as a preliminary means for creating interactivity. More precisely, if we follow the chains of causation in the loop between the various participants, we find that the algorithmic process is like a parallel world, only accessible through experimentation within the premises of this specific set-up. The mechanism's constructive preconditions stay hidden and become apparent only in the agency of its behaviour. Much of the critical effort within media art has been aiming toward bringing these conditions into discussion, making them accessible and contextualising them in the politics of things. This is a broad field, since the computational chain potentially pervades the situation just as much as cultural meaning passes through the networks of calculations. In order to reason about the conditions of interactivity, it is necessary to expose not only the model (be it process or result, or further chains of semiosis), but also the model formation within the interactive situation.

\section{Finding out}

Together with the concept of interactivity comes the notion of real-time. The fascination of self-regulation in the early cybernetic discourses is implicitly connected with a coming to life of a dynamic continuum of becoming. Also, over most of the 20th century, digital systems were somehow always too slow - the resistance to interaction took place in an interval between input and output. A continuous labour to integrate computational systems into the environment, for instance in the form of scientific simulations in the workflow of a laboratory or in the form of interactive music instruments, led to 
the reduction of the delay in the loop to the point that it became sufficiently short to give the impression of a neutral presence of time. Yet, simultaneously, measurement and display became fundamentals, which continuous or discrete interactive processes were to operate upon. More precisely, we can state that real-time interactivity is predicated upon the idea of the parameter. Pivoting on approximations of real numbers, real-time computation consists of a network of connected streams, whose immediacy is mediated through a parametrised mask of measured movement, sensor information; often, a graphical interface for such applications gives access to interaction points by means of images of sliders and wheels. More generally, a parameter-space is the implicit frame of reference in real-time interaction.

Interactive programming has been taking the complementary approach: instead of writing an interactive program that exposes continuous parameters at runtime, it exposes the activity of parametrisation itself, and more generally, the construction of programs at runtime. A starting point may be a very small formula - for instance a sound algorithm, which generates a process that, by converting it into an alternating current and playing it over a speaker, can be listened to. Instead of now thinking about what parameters need to be exposed to external change, and building an interactive application that can be used later, the formula that describes the process is rewritten directly. Changes of the program's time-map figure as the medium of interaction. Therefore, in such a situation, it is not so much the parameter space that is subject to experimentation, but the program text itself. More precisely, as we shall see, it is the different semantic levels of a program, which become thematic again.

In experimental mathematics, methods of interactive programming have been used to investigate the relation between a program text (a formal expression) and its output, which may be a set of numbers, or other formal expressions; within application design, such methods allow iteratively improve computer applications. Usually, each version of the program text simply expresses one such relation - one description corresponds to one result. In the conversational approaches of the 1960s for instance, an incomplete program would ask the programmer questions until the result was found. ${ }^{20}$ Now, as we saw before, this outcome may not have to be static. It also may be a process that exists only insofar as it unfolds and changes over time. In algorithmic sound synthesis, for instance, the program text describes something irreducibly situated in time; of course, one may record a sound wave and later play it back, jump around in it, or play it backward. Nevertheless, this does not touch the relation between an algorithm and its unfolding. Only after the fact, is everything data.

20 Matthews 1968; this procedure survives in today's terminal application, where program and programmer interact by turn-taking in the form of a dialogue. 
Taking a closer look at the structure of interactive programming, it becomes apparent that experimenting with inherently temporal results requires the relation between description and model to change significantly. Generally speaking, this is because the program as a description cannot consistently represent the changes to this description themselves. It turns out that should a program be rewritable at runtime, we are confronted with a paradoxical situation. If we start the whole computational process from the beginning each time something is changed, then the formula really can count as a valid plan of its unfolding. But each new onset means that the change itself is no longer situated in the moment it actually happens relative to the ongoing process. The world ends and is recreated. Another procedure is to divide the program into concurrent parts, each of which can be changed individually, but which may interact over time. Then, when a part is changed, it is the local unfolding of this new part that affects the rest of the system. This sounds like a good solution, because then, changes to the code happen in the context of a continuous behaviour of the system. But now we are confronted with the fact that the description does not reflect the behaviour as a whole anymore, since different parts of the system must be understood relative to their different points of departure. Moreover, looking at the program in its entirety, an assemblage of parts, it is not always clear whether they are a description of how to put together other parts or whether they describe the behaviour of such a part. ${ }^{21}$ As soon as one tries to integrate the rules into the interaction, their divergent interpretations (expression, process, or result) come into play. Of course it is possible, as a next step, to formalise the structural transition between different descriptions and represent those as the program. We then still have to decide which part of the text belongs to what part of the structure. Even more, the structure of these changes itself is again subject to the same problem if it is meant to be part of the interactive situation. So while some kind of segmentation is necessary, it is not generally decidable what belongs together and what is separate.

This symptom, which arises within interactive programming, allows us to consider the implications of unfolding on a more general level. As we have seen, the instrumental relationship between description and process, when it is supposed to allow an interaction in real-time, comes with a specific exclusion of the process of constructing the same system. If an immediate coupling

21 The moment of substitution of a part by a different part dissipates the general ambiguity of semantics of a whole program within its own parts. See e.g. Abelson/ Sussman 1996, ch. 1.1.5: "Despite the simplicity of the substitution idea, it turns out to be surprisingly complicated to give a rigorous mathematical definition of the substitution process. The problem arises from the possibility of confusion between the names used for the formal parameters of a procedure and the (possibly identical) names used in the expressions to which the procedure may be applied. Indeed, there is a long history of erroneous definitions of substitution in the literature of logic and programming semantics." 
of a system is desired, the laws that regulate this coupling cannot themselves be subject to interaction. In order to include these laws, not only has the idea of real-time to be relativised, but even more, the meaning of the system, the concept of a model becomes a matter in question. As long as we know exactly in advance what the meaning of a program is supposed to be (as long as we have a good specification, e.g. of its behaviour), however difficult, there is a possibility to line up the chain between description, computation and result, and construct an appropriate formalism. However, trying to find a new model requires a re-ordering of the whole situation, because interactivity with rules (and not with their parameters) entails a multiple split in the temporal domain; where time ceases to be a 'domain' that could be called 'real-time'. The ambiguity between things and their operative formation reappears here in form of conflicting levels of meaning, which can only partly be disentangled by situated decisions. ${ }^{22}$ Interactivity - maybe as opposed to interaction - turns out to be an open temporal antagonism, a differend ${ }^{23}$ on the verge of multiple temporal levels and multiple possible models. In a sense, this makes interactive programming thinkable as a miniature version of an experimental process on the border between formal and empirical methods, iterating between the different mechanisms of explanation, conjecture and failure. It exemplifies that when no cogent specification is given, and the subject of investigation is ambiguous, we cannot line up formalisation with interactivity in such a way that the former is only the necessary sacrifice to the technical, whereas real-time behaviour is authentic becoming. A situation in which it should be possible to find out something, the delimitation between thought and act has to be made amenable to reassembly. In other words, since it is not obvious to what degree the outcome is a construction of a new structure or a discovery within the current one, an investigation concerns the relation between free decision and strict deduction, or, from a different perspective, of the rational and the social. ${ }^{24}$

22 Inspired by Lyotard's article 'Time Today' (Lyotard 1991), in our paper 'Algorithms Today. Some Notes on Just-In-Time Programming, we have discussed this multiplicity of history in the context of a concrete system for interactive sound programming (Rohrhuber et al. 2005).

23 To give an example from empirical science: If we have an idea what parameters of some physical process may be relevant for a law, for some invariance, we can measure them (if we are lucky), and then test our equations against this data. Should either the experiment or the theoretical framework inspire us to see some other possible parameter, the measurement has to be done again. This is how the interaction between material culture of science and its concept formation is usually explained. (See e.g. Pickering 1995; Rheinberger 1997). In other words, prediction depends on the past, yet at the same time, the significance of past facts depends on their future effects. This causes interactivity to be necessarily situated in incommensurable orders, or incompatible law systems. In Lyotard's terminology, it can thus be considered a differend.

24 Longino 2001; already the early discussions on interactive programming show consequences that this has for the place automata should have in a process of reasoning. In the introduction to the proceedings of the conference Interactive Systems for 
The temporal aspect of this problem can be found in the relation between rules and their unfolding. Formally, it can be described as the relation between a structure and a model; more precisely, between some kind of formal deduction laws together with basic assumptions, the axioms, on the one side, and on the other, some mathematical domain, e.g. sets. Evidence suggests that formalisation is not only the necessary precondition for an interactive, experimental, empirical investigation; rather it is already part of this investigation. The construction of a logic requires mathematical assumptions. Or, for instance, within algorithmic composition, programming is not just a technique for building synthesisers, but is part of the compositional process. Similarly, in scientific operations, the schematism is not a precondition for empirical confirmation, but both are part of a new form. We have seen that this hybrid, this mutual implication between formal apparatus and empirical praxis is not simply a fusion. Rather, the structure of an investigation must be regarded as a paradoxical interaction between what is possible and what becomes possible by doing the possible. This "dialectic of formalization" 25 unfolds in a mutual determination of what is given and what is found out: "every creation of thought is in reality a creation of a new formalization and at the same time this new formalization establishes a relation or takes part in an interaction with the particularity of what we are trying to express." ${ }^{26}$

The process of finding out something operates in-between a discovery of something that previously existed, forming the conditions of research, and, at the same time, the construction of a new situation that did not exist before, but reconditions what can be constructed. ${ }^{27}$ The model is a linkage between a new possibility and a situated context in which it becomes unavoidable.

Experimental Applied Mathematics (Klerer/Reinfelds 1968), Klerer quotes Burton Fried, who had written one year earlier that the "utopian notion of a computer, which accepts the statements of a problem and automatically finds a way of solving it is clearly chimerical, save for those 'problems' whose structure has been thoroughly understood and for which methods of solutions are well known" (Karplus 1967, p. 169). Again, Klerer confesses that his own motivation, in contrast to the majority of the contemporary academic community, is "based on just such a utopian basis." He emphasises that, while the term interactive is difficult to define, "[...] we would expect more than in the old process of inputting a well-formulated set of directions with the machine performing in its capacity as an idiot servant." (Klerer/Reinfelds 1968, p. 9). I think that quite conversely, Fried's comment alludes to the basic incompleteness of a majority of formal systems, showing that exactly because we may extend a given system by a term that is not derivable from within it, interactive programming is interesting. One cannot decide in advance what part of the system will turn out to be involved in such a change; this suggests interactive programming (of whatever kind) as an interesting conceptual alternative to interaction with a program that has its interaction point already defined in advance. So problem solving is indeed "chimerical", since it involves ambiguous agencies, which explains the need to include the programming activity itself into the program.

25 Badiou 2007, pp. 90-92

26 Badiou 2007, pp. 90-91

27 In this, the dialectics of formalisation are equivalent with Badiou's later concept of a truth procedure. Following Cohen's mathematical technique of forcing, Badiou is able to 


\section{Traps revisited}

Computation follows a strict protocol laid down in its description, yet at the same time gives rise to completely unexpected things. This is why an algorithm may, under some circumstances, occupy an intermediate position between a law as discovery (when we find it, we suppose that it has always existed) and the law as a constructed artefact (which is an intervention into what exists). ${ }^{28}$ It becomes a hybrid between the ghost of the programmer, stood in for by the automaton and the autonomy of a new situation.

Let's return to the question in how far interactivity of this kind is structured in analogy to a trap. What is caught in an algorithm? Who is it an agent for? I think the interesting central thought that Gell started out with - namely that in some way, the trap is a model of both hunter and hunted - is useful for clarifying the situation of a 'dialectics of formalisation' that implies some paradoxes of interactivity. In his investigation of agency, Gell is able to show that certain situations and artefacts cause the observer to enter into a process of reasoning. ${ }^{29}$ Their questionable mode of fabrication, the unclear origin, maybe we can say their artificial and alien character, causes them to force an attribution of agency. "Is this spot here on purpose or did it just happen unintentionally?" or "Is this strange sound we just heard part of the composition, or is it a mistake of the performer?" Agency in this sense is essentially an open question provoked by a disturbance of conventional inference, a question which can only be answered by hypothetical reasoning, or, as Gell puts it, abduction of possible originations. This search for the inner logic of a situation enmeshes the participants in possible alternative worlds of causal, and thus, temporal connections.

In a peculiar dialectics, agency is what an observer infers of a phenomenon's origin, and simultaneously it is the power to induce this reasoning. Like the trap, the artwork is a disturbance of the causal milieu in which it is situated, ${ }^{30}$ a disturbance that opens an explanatory gap, a cognitive dissonance. We can say it is a different model of cause and effect within a given reference frame, a model that forces one to hypothesise about possible explanations (this abduction is the derivation of a law from a model). In this way, certain artefacts are able to induce interactivity - interactivity as a process

show ontologically under what conditions a new formalisation is possible in a given situation (Badiou 2007b). For an investigation of a relation between set theory and agency theory in this light, see Rohrhuber 2008.

28 The issue unfolds in a contradiction between place, finder (an investigation), and ownership: does the found object belong to the place or the finder? Does the place belong as much to the landlord as the object belongs to the finder? Does observing an event and investigating its traces set a rupture that contradicts the continuity of territory?

29 Gell 1998

30 Gell 1998, p. 20 
of reasoning, of situated thought. That human beings may make such inferences is without question. But it is more interesting to ask what could be the conditions under which a new formalisation, a new causation may appear at all. As we have seen, in such a rupture, social and natural causes become mutually exchangeable, just as much as the difference between construction and discovery of reality have to be negotiated anew. So if an artefact, or more generally, a situation as a whole may be the cause of such a shift, how does it have to be structured? What is a model for finding models?

As a conceptual starting point, traps have turned out to be interesting as an epistemic model; they allowed us to consider something like an objectified anticipation together with an objectified ignorance. ${ }^{31}$ At first, this was thought as two parts: an open conjecture and an automaton, a 'materialised theory' on the one side; and the unfolding scene of captivation on the other, where the enclosure must have already been entered before it snaps shut. It is in evidence that these, in turn, imply two concepts of time; in the first, the absentation of the hunter causes the prey's possible presence. In the second, the prey is already caught before it realises this fact. Like in a weir, for a fish there is no point where the difference can be found between inside and outside. In other words, in order to discover something, and not invent it, this entity must have some autonomy; however artificial the situation, it must show itself. Yet it must show itself in the situation that is given already. However if it is not certain what is to be caught, this separation becomes unstable, and as a consequence, the trap begins to resemble an experimental system. Here, the model breaks; hunter and prey become indistinguishable. Anticipation becomes a conjecture about a possible new situation in which we are entangled already.

Thus, the trap remains a model for the possibility of finding out (something). It suggests that it is the dynamics of laws that abstract from immediacy and allow experiment: the inherent temporal logic here is its formal indifference to time; just as abstraction allows statements not to differentiate between certain things, the abstract also shows indifference to the moment at which events occur. As we have seen, this is what made a program imply both possible actions and their formalisation, both operation chain and plan. But instead of giving rise to interactivity in the sense of a presence of unification, it has turned out that abstraction - if it is, despite all contradiction, included in the situation - leads to a resilient and antagonistic assemblage.

31 For Blumenberg, quite in accordance with the view Gell proposes, traps are intimately related to the peculiar temporality and agency in concept formation. He considers preemption in its dialectics between absence and presence: "The trap acts in place of the hunter in the moment of his absence, but in the prey's presence. These conditions are revealed to be the reverse in the trap's production. It is the reified expectation. Insofar, the trap is the first triumph of the concept [Begriff]." (Blumenberg 2007, p. 14, my translation). 
Suspending direct access, a formalised situation may itself impel the process of a different form. The abstraction from the trap allows us to maintain that hypothetical situations do exist: they are not just a product of an observer, opposed to a non-hypothetical world. There are cases where abduction is to be found within the situation - finding out such cases is itself a matter of formal experiment (it must then be possible to find something that was not even hidden). 


\section{References}

Abelson, Harold/Sussman, Gerald Jay (1996): „Structure and Interpretation of Computer Programs«, Second Edition, Cambridge, London: MIT Press. Online available: <http: / / mitpress.mit.edu/sicp/full-text/book/book.html> (last access 6. July 2008).

Andersen, Peter Bøgh (2003): »Semiotic Models of Algorithmic Signs«. In: KarlHeinz Rödiger (Ed.), Algorithmik - Kunst - Semiotik, Heidelberg: Synchron Wissenschaftsverlag der Autoren, 165-193.

Badiou, Alain (2006): Briefings on Existence: A Short Treatise on Transitory Ontology, Albany: State University of New York Press.

Badiou, Alain (2007): The Concept of Model: An Introduction to the Materialist Epistemology of Mathematics, Melbourne: re.press.

Badiou, Alain (2007b): Being and Event, London: Continuum.

Blass, Andreas/Dershowitz, Nachum/Gurevich, Yuri (2008): »When are two algorithms

the same?«. Online available: <http://lambda-the-ultimate.org/node/2729> (last access: 6. June 2008).

Blumenberg, Hans (2007): Theorie der Unbegrifflichkeit, Frankfurt a.M.: Suhrkamp.

Callon, Michel (2005): »Why Virtualism Paves the Way to Political Impotence. A Reply to Daniel Miller's Critique of Laws of the Markets«. Economic Sociology 6(2), 3-20.

Faust, Kristine (2003): Wem gehört Neuschwanstein?, München: Aviso, Bayerisches Staatsministerium für Wissenschaft, Forschung und Kunst.

Gell, Alfred (1998): Art and Agency: An Anthropological Theory, New York: Oxford University Press.

Gell, Alfred (1999): »Vogel’s Net«. In: Alfred Gell, Eric Hirsch (Eds.): The Art of Anthropology: Essays and Diagrams, London: Berg Publishers, 187-214.

Gell, Alfred (1999b): »The technology of enchantment and the enchantment of technology«. In: Alfred Gell, Eric Hirsch (Eds.): The Art of Anthropology: Essays and Diagrams, London: Berg Publishers, 159-186.

Haraway, Donna (1988): »Situated knowledges: The science question in feminism and the privilege of partial perspective«. Feminist Studies 14, 575-599.

Iverson, Kenneth. E. (1979): »Notation as a tool of thought«. In: Communications of the ACM, 23(8), 444-465.

Karplus, Walter J. (1967): On-Line Computing, New York: McGraw-Hill Education.

Kay, Alan C./Goldberg, Adele (1977): »Personal dynamic media«. Computer 10(3), 31-44.

Klerer, Melvin/Reinfelds, Juris (1968): Interactive Systems For Experimental Applied Mathematics, New York: Academic Press.

Kneer, Georg/Schroer, Markus/Schüttpelz, Erhard (Eds.) (2008): Bruno Latours Kollektive. Kontroversen zur Entgrenzung des Sozialen, Frankfurt a.M.: Suhrkamp. Knuth, Donald. E. (1992): Literate Programming, Stanford: Center for the Study of Language and Information. 
Krämer, Sybille (1993): »Operative Schriften als Geistestechnik. Zur Vorgeschichte der Informatik«. In: Peter Schefe (Ed.): Informatik und Philosophie, Mannheim: BI-Wissenschaftsverlag, 69-84.

Latour, Bruno (1993): The Pasteurization of France, Cambridge: Harvard University Press.

Leroi-Gourhan, André (1964): Le geste et la parole (2 Volumes), Paris: Albin Michel.

Longino, Helen E. (2001): The Fate of Knowledge, Princeton: Princeton University Press.

Lyotard, Jean-François (1991): Time Today, Stanford: Stanford University Press.

Pickering, Andrew (1995): The Mangle of Practice: Time, Agency, and Science, Chicago: University of Chicago Press.

Rafaeli, Sheizaf (1988): »Interactivity: From new media to communication«. In: R. P. Hawkins/J. M. Wiemann/S. Pingree (Eds.): Sage Annual Review of Communication Research: Advancing Communication Science: Merging Mass and Interpersonal Processes 16, 110-134.

Rheinberger, Hans-Jörg (1997): »Experimental Complexity in Biology: Some Epistemological and Historical Remarks«. Philosophy of Science 64, Supplement, 245-254.

Rohrhuber, Julian (2008): »Mengenlehre«. In: Ilka Becker/Michael Cuntz/Astrid Kusser (Eds.): Unmenge: Wie verteilt sich Handlungsmacht?, München: Fink Verlag, 141-156.

Rohrhuber, Julian/de Campo, Alberto/Wieser, Renate (2005): »Algorithms today Notes on Language Design for Just in Time Programming«. In: Proceedings of the 2005 International Computer Music Conference, San Francisco: International Computer Music Association, 455-458.

Roßler, Gustav (2008): »Kleine Galerie neuer Dingbegriffe: Hybriden, Quasi-Objekte, Grenzobjekte, epistemische Dinge«. In: Georg Kneer/Markus Schroer/Erhard Schüttpelz (Eds.), Bruno Latours Kollektive: Kontroversen zur Entgrenzung des Sozialen, Frankfurt a.M.: Suhrkamp, 76-107.

Schüttpelz, Erhard (2008): »Der Punkt des Archimedes. Einige Schwierigkeiten des Denkens in Operationsketten«. In: Georg Kneer/Markus Schroer/Erhard Schüttpelz (Eds.), Bruno Latours Kollektive: Kontroversen zur Entgrenzung des Sozialen, Frankfurt a.M.: Suhrkamp, 234-258.

Serres, Michel (1987): Der Parasit, Frankfurt a.M.: Suhrkamp.

Spurný, Pavel/Oberst, Jürgen/Heinlein, Dieter (2003): »Photographic observations of Neuschwanstein, a second meteorite from the orbit of the pribram chondrite Nature 423, 151-153. 


\section{UNORT- \\ KATASTER}

AN

URBAN

EXPERIMENT

TOWARDS

PARTICIPATORY

MEDIA

DEVELOPMENT

Georg Trogemann,

Stefan Göllner,

Lasse Scherffig 


\section{Introduction}

While in the past the predominant goal of software engineering was the efficient development of robust, reliable and easy to use systems, the state of affairs seems to change radically at the moment. On the basis of gained experience and acquired adulthood, a significant number of users ask for empowerment. They not only want to be asked afterwards what they like or dislike about an application, but actively participate and get a say at all stages of development, right up to the questions of profit-sharing. Therefore, the recently checked out software design methodologies primarily try to come up with strategies for participation on all levels of the software process. Packaging and shelving of software seems to be a discontinued model, the new software development processes try not to end with the first deployment of a system but stay open to the requirements of an ever-changing context and the continuously upcoming needs of users. To achieve such systems we need open software architectures on the one hand, and the active involvement and the energy of participating people on the other.

The development of the Unortkataster, which will be discussed below, is driven by the intention to create an online tool in collaboration with users that facilitates controversial thinking about critical places (Unorte) in an urban environment (the area of the City of Cologne). An "Unort" is a marked place or area on a map, added by text-descriptions and other illustrating media by one author or a group of authors. The term "Unort" refers to any urban place or situation, which is criticised for a special lack of quality - based on individual settings. The tool is intended to moderate discussions about these kinds of places and organise them by temporal and spatial aspects. In the following we analyse what potential the approach of 'technology probes' delivers for participative software development in the context of big cities. This entails the question, which possible map-based, community applications may be applicable in the interleaved domain of physically localised public space and a globally networked public sphere?

In the following, we also try to illuminate another quite important - normally underestimated - aspect regarding participatory software systems. Once developed and implemented, software acts independently from their creators. It becomes an active player in the social communication game. Algorithmic processes are self-operating entities that actively change the domain in which they perform. This viewpoint does not focus on the cultural memory and/or its well-investigated, modern realisation as digital archive. Rather it is about the underlying algorithms that select, copy, transform, compare, visualise, and permanently reorganise the digital information and its connected communication processes. Thus, due to their invisibility and despite their omnipresence, algorithms are probably the most underrated artefacts of our days.

\section{5}
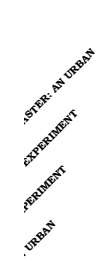
It seems to be time to ask a basic question: How do software algorithms actively shape society, its knowledge, and communication patterns?

\section{Societies and their technologies}

In his much-cited book Myth of the Machine, Lewis Mumford convincingly demonstrated that human culture is not - as often stressed - mainly a result of man's ability to build and command tools, but that tools could only develop so far because of a significant series of inventions in ritual, language, and social organisation. ${ }^{1}$ In fact, the first complex machines in history were not mechanical entities but composed of living humans, each assigned to his special office, role, and task, that led to - even under contemporary criteria - tremendous work performances (e.g. the pyramids, palaces, town walls, etc.). Because the components of the machine were working in separated locations, although their interplay implemented an overall functionality, Mumford called them invisible machines. The labour machines were merged with the destructive military machine and the controlling administrative machine to the megamachine of the early totalitarian states. Within this system we encounter the very first, really powerful machine operator in history. In this image, the whole society is the machine and the god-king has complete control over the machinery. "But only Kings, aided by the discipline of astronomical science and supported by the sanctions of religion, had the capability of assembling and directing the megamachine." So the myth of the machine and the cult of divine kingship rose together, as Mumford puts it.

The invisible machine of the ancient divine kingship was only manageable because of its strict hierarchical organisation. And hierarchy is still the most powerful and important principle we have at our disposal to realise reliable and controllable complex machinery. Thus it is not surprising that we also find this principle within the most complex (and maybe most powerful) machinery of our times, the computer. From an engineering point of view, the computer is only manageable because of its consequent hierarchical organisation. But what has been adopted from past totalitarian states and still is valid on a technological level has completely changed on the social level where the computer becomes effective. The organisation of society, the whole socio-economic and political structure as well as the accompanying power relations, is today based on the (more or less) free decision of participating humans and therefore completely different to the times of divine kingship. But also freedom can be considered as part of a computable function, as it was attempted by Stafford Beer in his failed experiment of the seventies to

1 Mumford 1966 
transform the whole Chilean political economy into a self-organising, cybernetic machine. ${ }^{2}$ The substitution of hierarchy by self-organisation was an important paradigm shift in the modelling of societies.

According to the sociologist Manuel Castells, we now live in the Network Society, which also only marks a transient state in an ongoing process of a renewed radical change of the power relations. "Cultural battles are the power battles of the Information Age. They are primarily fought in and by the media, but the media are not the power-holders. Power as the capacity to impose behaviour, lies in the networks of information exchange and symbol manipulation, which relate to social actors, institutions, and cultural movements, through icons, spokespersons, and intellectual amplifiers." ${ }^{3}$ This means that in the upcoming society, power does not simply disappear but becomes inscribed in the cultural codes through which people and institutions represent their interests and arrive at decisions. The most important cultural code in which power will be inscribed is probably software code.

What we should learn from Mumford, Castells, and others and what is still not sufficiently considered within the software engineering faction, is the insight that major technological progress always goes hand in hand with social dynamics. We can no longer restrict the scope of our software development projects to the characteristics and technicalities of the intended applications, but have to consider the whole social universe in which the application will function. The necessity to consult social aspects and the needs, life-styles and desires of people during software design processes is also supported by another novelty of the software design problem. This has to do with missing experience in our dealing with computational objects. "When designing a classic object such as a chair, there are long traditions embedded in the practices of designing and using chairs that are not easily escaped. When designing a computational thing, however, not only the object but frequently the entire object category will be new to us. The effect is that our understanding of the objects we set about designing is extremely limited. Lacking such a fundamental understanding of the object, studies of possible situations of use, the needs and desires of potential users, and methods from other domains of practice have become tools for navigating an unfamiliar design space." ${ }^{4}$ Thus engineers who participate in the design and development of the new technologies will constantly - consciously or unconsciously - have to construct hypotheses and expectations about their users and the social context in which the technology will be used. Involuntarily, software

Pias 2004

Castells 1998, p. 335

4 Mazé/Redström 2004 
engineers thereby become sociologists, or engineer-sociologists as Michael Callon calls them.

All these arguments lead to the conclusion, that, if we want do develop participatory media where people take over substantial parts of the responsibilities for the vividness and progress of the whole application, we have to find answers to some fundamental questions. Some of these questions are: What are the important contemporary rituals, the rules of communication, and the organisational forms of the involved communities and institutions? How does the social fabric of modern communities work and what is the role of software in this network? How can software show its own social conditionality and the wide influence of the subjective perspectives of the participants? How does software become effective in reality and change the communication and knowledge of the social environment in which it is operating (e.g. the Google page rank algorithm)? How can software remain open to the variations of the context in which it is performing and to the changing and proceeding needs of the users?

\section{Urban investigations and participatory processes in urban design}

In order to find answers to some of these questions the city appears as the condensed laboratory of society where changes become evident and future developments can be anticipated. The city still represents the most successful form of human co-existence and has resisted many announcements of its crisis. Contrary to former assumptions, communication technology can be regarded as a stabilising factor for the physical structure of the city. "The physical synergies between telecommunications and physical networks mean that both tend to concentrate in the same city centres and the same corridors between them." 5

Condensation and the related diversification is an old motive for the appeal of the city and has a crucial impact on the social structure. Cities do not provide a homogenous environment where people share the same attitudes at the same location. Instead, people of different ethnic and cultural backgrounds coexist and are able to live in close proximity to each other without being confronted directly. The guarantee to maintain differences in spite of the lack of geographic distance seems to have become a particular phenomenon for the modern city society. ${ }^{6}$ Moreover, the city allows the individual or community to erode the mechanisms of communicative constraints that are character-

\footnotetext{
5 Graham/Marvin 1996

$6 \quad$ Siebel 2004
} 
istic for rural societies. ${ }^{7}$ The city in this sense should be regarded as space where people might start talking to each other but might also refrain from doing so, as Dirk Baecker puts it. ${ }^{8}$ The containment of the citizen contradicts efforts to change city culture by communication systems in order to enforce understanding among citizens and create a feeling of togetherness. Normative theories of urbanity therefore polarise communications into a "for or against" and try to enforce a consensus for one of the alternatives. ${ }^{9}$ However the city as a physical structure is characterised by the production of conflicts that touch questions which go beyond the individual sphere. Wikipedia describes a community as "a social group of organisms sharing an environment, normally with shared interests." While in online communities shared interests are indeed the dominant connector, in cities the shared physical location becomes the other dominant condition for community building. Civic communities in this aspect are not primarily connected by shared similarities but by shared problems.

However, the process of community building in real space evolves much differently from how it happens in an online environment. Online communities have begun to conquer the former, exclusive territories of the city: portals like MySpace or Facebook have started to compete with the street or the mall as the preferred place to "see, be seen, and connect". However, community building in the city may emerge deliberately as much as accidentally. Communities therefore evolve because people share the same housing areas, the same thoroughfares, or the same recreation spaces. In addition the relations of communities are changing over time or may even be time-based: people joining a traffic jam, people sharing the experience of a noisy street, people being involved in the effects of a natural catastrophe etc. Environmental aspects become connecting points as well as infrastructural connexions. Participative processes allow a better understanding of how the character of those communities is changed by the confrontation of physically localised space with a globally networked space.

An early forerunner of participatory city-design, Kevin Lynch, tried to involve people into city planning processes. For that purpose he used simple but effective instruments: He asked citizens for sketched mental maps and did interviews at the same time to better understand the relation between the participants maps and the explanations. By using these very simple and communicative methods he tried to understand how the individual image of an environment is constructed. He aimed at a better understanding for the "collective image”, which should finally lead to better city-design methodologies.

\footnotetext{
7 Luhmann 1997

8 Baecker 1994

9 Schroer 2006
} 
In his book The Image Of The City he summarises the requirements for an improved image: "The image should preferably be open-ended, adaptable to change, allowing the individual to continue to investigate and organize reality. There should be blank spaces where he can extend the drawing for himself. Finally, it should in some measure be communicable to other individuals." 10

Lynch's concepts have remained a challenge because the act of designing a city from the very beginning is a rare possibility. Architecture proceeds in a dictatorial ${ }^{11}$ way because it forces the city into a structure of temporal persistence. The built structure is static and resists being adjusted according

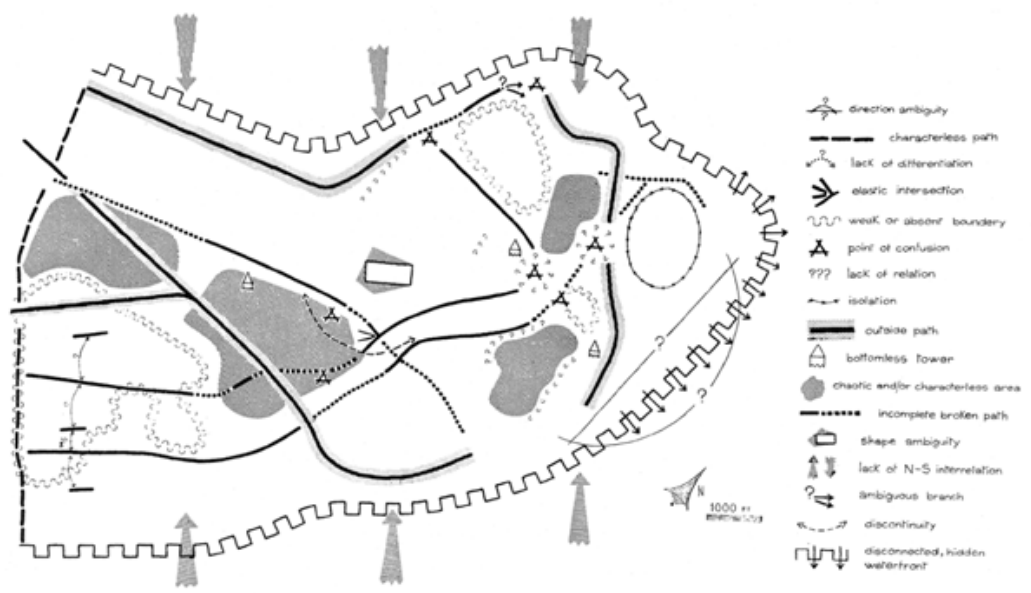

Fig. 1. Kevin Lynch's analysis of "problems of the boston image "resulting in a mental map (Kevin Lynch (1960): The Image of the City, Cambridge: MIT Press, $p$. 24, Fig. 8)

to changes in society. New architectural and city planning concepts do not lead to flexible structures that allow people to modify environments related to changing life conceptions.

But the requirements Lynch verbalised for the rebuilding of cities can be read as a rationale of how networked communication systems are used by citizens today and which change the perspective of how openness of the cityimage might be established by building into software ${ }^{12}$. Communication networks are working on top of built city structures and allow interaction inde-

10 Lynch 1995, p. 9

11 Mersch 2000

12 Rötzer 1998 
pendently of physical barriers. Cell phones give the most obvious example of how this is influencing the behaviour of the citizens: making phone calls without depending on temporal and spatial constraints changes the relations between participants as well as the relations between public and private territories. Shopping by the Internet allows not having to go to real shops and leads to new business models. Positioning systems suggest alternative routes for road users and changes traffic. Openness under these conditions means that the built structure loses its character as a communicative barrier: People's activities and communication are liberated from the conditions of real space by the flexibility of the network. But to get an impression of how these changes influence today's citizens' image of the city, a proper analysis of impressions and opinions that are related to it would still be necessary.

The mental maps that Kevin Lynch used to collect input from citizens during his investigations are becoming effective again in the context of cityrelated, online community tools. Transformed to software, they can become a powerful tool for the involvement of citizens in the process of finding common requirements for future developments.

\section{Software in social context}

This section will roughly describe our approach to software design that we pursue in the development of the Unortkataster. As a process of software development, this approach must partially rely on the history of designing both software systems and their interfaces. From this history we borrow ideas and methods and apply them within the context of participatory media and therewith the perspective of the engineer-sociologist.

Firstly, to build such a participatory medium we need a strong engineer approach that not only leads to robust, reliable, and easy to use software architectures, but also allows for an easy adaptation to ever changing social contexts and the accompanying drift of user needs and expectations. Here we have to fight a serious paradox. We know that the context of the application will change, but we do not know how it will change. Nevertheless, our software has to be prepared for it. To encounter this problem we try to transform and apply laws from Software Evolution. Secondly, we need transparent strategies of communication, moderation, documentation, and decision, which allow user participation during all stages of development. This entails the problem that the processes organised by the system must be understood in their changing subjective and social dimension and context. Thirdly, this involvement has to start before the first ideas are brought down to paper or any decisions about the application are made. This usually generates another paradox: Potential users (this term is already difficult) in most cases have no 
clue how novel and innovative participatory media could look like, but that is exactly what we ask them for. Designers and users likewise need inspiration that is driven by technology and its possibilities as well as the needs and practices of its situated users.

We may roughly cluster these problems into three categories: 1) engineering, 2) participation, and 3) inspiration. The following brief history of interface design is oriented along these three terms.

\subsection{A brief history of interface design}

For software to become effective in reality in an immediate sense, an important technological change had to occur: the introduction of the interrupt. The interrupt constitutes a computer's ability to react to new input during program execution. Although it has accompanied computing machinery since the late 1940s, computer science still struggles with the problems it poses for the theory of computation, as the theory of Turing Machines does not generally account for such interventions. ${ }^{13}$ For the application of computing machinery, the interrupt established for the first time a real-time coupling between computing machinery and the world. Another change taking place around the same period similarly shaped what interactive computing remains until today: with the introduction of digital computing unlike analog (computing and other) machines, the relation of digital data to its representations became arbitrary, or in terms of semiotics: not longer indexical but symbolic. ${ }^{14}$ Since then, the symbols through which users may perceive and act upon an interruptible computer have a two-sided nature: they are sign and signal - open to interpretation but at the same time causally effective in a machine. ${ }^{15}$

Following these changes, operators operating computing machinery became users wielding tools. With that, a science of users and interfaces emerged that tried to solve the problems raised by the changes, a science that from the 1980s on was called human-computer interaction or HCI.

\subsubsection{Early HCI: Engineer and test}

As the construction of computing machinery was an engineering activity, HCI was strongly influenced by engineering disciplines. But since the interrupt allowed the incorporation of the user into computations, it was

\footnotetext{
13 Wegner 1997

14 Pias 2000, p. 45

15 Nake 2000
}

\section{0}


also, from its very beginning, a science of people. It hence co-developed with another young, scientific discipline trying to understand the human mind: cognitive science (and artificial intelligence). And while cognitive science was largely influenced by work on logic and hierarchical problem solving, human use of interfaces was seen as a process of goal directed problem solving, too - a process that could be modelled and calculated in order to build ideal interfaces for the "human processor". ${ }^{16}$

For the engineer, who since the days of divine kingship has been working with the principle of hierarchy, the process of creating software could likewise be organised as a process of hierarchical problem solving, starting from requirements defined in the beginning, and ending with a product that fulfils these requirements. The model that, until today, is associated with this process for its hierarchical, top-down structure was named the "waterfall model" 17 . Since the requirements of an interface can only be fulfilled in conjunction with a user, soon usability tests with users were introduced - a method later named usability engineering.

Because of the close connection of HCI, cognitive science and artificial intelligence, it is not surprising that the first influential criticisms of the way user and interface were conceptualised stem from books that were written as contributions to the artificial intelligence discourse. The book Understanding Computers and Cognition ${ }^{18}$ attacked the assumptions much work in artificial intelligence rested upon by confronting this "rationalistic tradition" with Heideggerian hermeneutics and speech act theory. Thus the authors stressed the problems implied by context dependency and situatedness. The subtitle of the book already suggested the role it should later play, outside the artificial intelligence discourse, when it was adopted by HCI researchers: "A New Foundation for Design".

Similarly Lucy Suchman in her book Plans and Situated Action also used Heidegger's terminology to show that human actions and goal-directed problem solving rarely coincide. ${ }^{19}$ Her theoretical considerations were backed up by a detailed empirical study of the interaction of users and an intelligent machine. This analysis was based on the methods of ethno-methodology. Here, too, what was introduced to the artificial intelligence field as a means of criticism was soon taken up by the HCI community and used as a design method.

16 Card et al. 1983

17 Denning/Dargan 1996, p. 109

18 Winograd/Flores 1986

19 Suchman 1987 


\subsubsection{Understanding situated use: Participatory design}

The critique formulated by Winograd and Flores or Suchman with its focus on understanding the situated human was paralleled by a development in Scandinavia: Here during the 1970s the participatory design tradition was born.

Participatory design emerged from a special historical condition: during the 1970s, $90 \%$ of the Scandinavian workforce were organised in unions. Consequently, the unions were granted influence on large parts of what determined their members' working conditions. In Norway, a co-determination agreement was signed allowing worker participation in the development of new workplace technology. ${ }^{20}$ Central to the early projects on co-determination was the Marxist notion of ongoing conflict between capital and labour, as well as approaches that tried to understand computer systems as sociotechnical systems. ${ }^{21}$ Against the observation that "democracy stops at the factory gates" 22 the vision of workplace or industry democracy was developed. ${ }^{23}$ The power relations within the factories were to be rebalanced and this had to include incorporating the workforce into the overall design of their workplace.

In a series of projects in Norway, Sweden and Denmark, computer scientists and their prospective users developed new technology for several areas of industry. These projects first of all led to new methods of how to enable users to participate in the design of new technology as it soon became clear that just facilitating conversation between designers and prospective users in order to establish requirements was not enough. ${ }^{24}$ Tools were needed that would bridge the gaps between designers and users, allowing each to access the tacit and contextual knowledge of the other. Therefore, methods such as role-playing with mockups - non-functional, low-fidelity prototypes made, for instance, of cardboard - became an important design method. ${ }^{25}$ Prototypes may here be seen as artefacts that provide a common language for designers and users, enabling and structuring discourse across cultural boundaries.

This "Scandinavian challenge" yielded "a US response" 26 that unlike the Scandinavian tradition was not fueled by Marxist ideas but by market

\footnotetext{
20 Kuhn/Winograd 1996, p. 290

21 Kuhn 1996, p. 284

22 Spinuzzi 2002

23 Kuhn 1996, p. 284

24 Kuhn/Winograd 1996, p. 291

25 Kuhn 1996; Muller 2002

26 Spinuzzi 2002
}

\section{2}


demands, where "the result of a good design is a satisfied customer." ${ }^{27}$ The methods developed within participatory design projects here proved to satisfy customers (or management representatives) just like workers' unions in Scandinavia. Participatory design hence became one major set of tools within the toolbox of HCI. However, as Clay Spinuzzi argues, corporate participatory design reinforced a division of labour between designers and users that contradicted the emancipatory ideas of workplace democracy: ${ }^{28}$ One attempt, for instance, to treat user participation from an engineering perspective is the use of "personas". ${ }^{29}$ These are abstract archetypical members of the target group based on observation but defined by the designers. Personas, according to Kari Rönkkö, include the user's perspective into the development by at the same time excluding the user from major parts of the work. ${ }^{30}$

At this point Peter Denning and Pamela Dargan draw a general line through the HCI field dividing it into two parts: On the one hand they see engineering approaches, including software engineering and the cognitive science inspired work for which design leads to products that fulfil specifications. On the other hand there are "user-centred approaches" focusing on situated action and yielding satisfied customers. ${ }^{31}$

\subsubsection{Getting inspiration: Artistic practices}

While one may follow Denning and Dargan and argue that the design of computer systems evolved between engineering and user-centred design, other ideas developed, too. HCI has repeatedly been seen as a field in which spaces of action and articulation are defined because design defines "the space of what can be said." ${ }^{2}$ With the interface seen as a social and therewith relational space, the discourse opened for inputs from a new field: artistic practices were taken up, and in opposition to the "engineer-designer" the idea of the "artist-designer" was promoted.$^{33}$ At the same time, large parts of the HCI community attempted to change the name of the field into "interaction design" 34 .

In a discourse that already treated interfaces as spaces for communication, ideas from art forms that had already concentrated on space and

27 Denning/Dargan 1996, p. 110

28 Spinuzzi 2002

29 Cooper 1999

30 Rönkkö 2005

31 Denning/Dargan 1996, p. 108

32 Winograd/Flores 1986, p. 78

33 Smith/Tabor 1996

34 see e.g. Preece et al. 2002 
communication could have a major influence. The filter through which these ideas entered the HCI discourse again was space: within a project that aimed to develop interfaces for urban and rural public space in Italy, Norway and the Netherlands, interventionist strategies were applied to the interface design problem. ${ }^{35}$ Artistic maps, disposable photo cameras, albums and postcards were given to the target group (elderly people in this case) and understood as "cultural probes" that were to create visual and textual response. From such subjectively sampled material, prototypes and situations were constructed and again confronted with the audience. Finally, interventions that artistically treated the results of the process were suggested.

The official genealogy of Gaver and Dunner positions this approach in a line with situationism (especially, of course, psychogeography) answering the questions about the situated use of new artefacts with situationist strategies. However, cultural probes implicitly recalled some participatory design projects in which already methods such as end-user photography and storytelling had been applied. ${ }^{36}$

For the HCI field the probes promised to be able to answer a fundamental question that neither engineering nor user-centred design approaches could handle. Namely, what applications should be developed for novel technologies? This is a question that historically proved hard to handle because on the one hand users tend to use new technologies in an unintended way, and on the other hand users in participatory design sessions are likely to suggest applications they already are familiar with. The HCI practice hence depends on methods to "identify needs" of prospective users ${ }^{37}$ and to forecast their use of what is offered them. One method to deal with this problem by studying such "possible situations of use" 38 has been developed in marketing research. It consists of a marketing analysis focusing on "lead users" - avant-garde users that are already familiar with new technologies and that therefore may serve as a "need-forecasting laboratory." 39

With similar goals the cultural probes approach was taken up by other researchers from the HCI field. In order to not only probe the context of the intended users but to directly involve them in the design of new technologies, the concept was extended creating "technology probes." These, as opposed to non-functional mockups, are functional prototypes using new technology and are deployed in real-world situations where they observe their own use. ${ }^{40}$ Hence 'technology probes' not only provide a common language enabling par-

35 Gaver/Dunner 1999

36 Muller 2002

37 Preece et al. 2002, p. 201

38 Mazé/Redström 2004

39 von Hippel 1986

40 Hutchinson et al. 2003

\section{4}


ticipation, but also become active players in the language game they are part of. Remarkably, all the projects working with probes resulted in designs that primarily wanted to create possibilities of communication.

\subsection{How software makes a difference}

Software which operates in social contexts needs continual management during its whole life cycle. Software engineers have simultaneously to track and reconstruct the social context upon which the software is acting. That is the only way to make sure it keeps its validity and does not progressively lose acceptance (see Laws of Software Evolution below). This requirement is due to the fact that software changes its own operation domain and causes a growing mismatch between the model of its domain, which is implemented in the software, and the domain itself. From a general perspective we have different agents acting in the same space, such as individuals, communities, institutions, texts, concepts, programs, buildings, and other sorts of artefacts that mutually influence each other. From the Actor-Network-Theory of Bruno Latour (and others) we know that in such networks humans are not the only ones who act. According to its theory, the Actor-Network links together elements (and all the elements of successive links) to a network of mutual influence that performs as a whole. The Actor-Network-Theory, which treats humans and artefacts symmetrically, tries thereby to explain how material-semiotic networks generate certain results and behaviour as an integral entity. One interesting aspect about this theory is that it rejects the naive view of technical artefacts or humans existing prior and independently of their participation in the social and semiotic network of interactions. Accepting this line of argumentation, we also have to regard software as an acting entity within a complex network. In general, software is linked to many other agents, thus it becomes effective on mainly two levels: ${ }^{41}$

- mental: programs objectify abstract ideas. They implement concepts that become part of daily life. The users are forced, whether they want or not, to deal with this new reality, interpret it and integrate it in their own subjective way into their view of the world;

- auto operational: as self-executing entities, programs have an instantaneous effect on reality. For example, robots, via their effectors, can directly manipulate their own environment. But such self-operating systems in general, if they are working in a social context, change the conditions of their operation since they open and close options for

41 Floyd/Klischewski 1998 
alternative choices. They are directly connected to the action space of the participating users and actively alter it.

\subsubsection{Models and E-type applications}

Models are of central importance in the whole field of computer science. Consequently, during the common software development process different sorts of models are also developed and deployed. To achieve a deeper understanding of the complex connections between software and its application domain, we have to briefly inspect modelling theory. The key properties of the general concept of a model are according to: ${ }^{42}$

\section{Mapping property}

Models are always models of something, namely mappings, i.e. representations of natural or artificial originals, which themselves might be models. The concept of a mapping coincides with the concept of assigning model attributes to original attributes. That means mapping is based on the mathematical definition of mappings;

\section{Reduction property}

Models in general never capture all attributes of the original they represent, but only those which seem to be relevant for the creator or user of the model;

\section{Pragmatic property}

Models are not per se unambiguously assigned to their originals. They fulfil their function of substitution a) for specific - recognising, and/ or acting, model-using - subjects, b) within a certain time interval c) under the restriction to certain mental or actual operations.

According to Meir M. Lehman, under the perspective of modelling, a program "is a model of a model within a theory of a model of an abstraction of a portion of the world or of some universe of discourse." 43 This quote points out that in software engineering at least three models are at work: the application model (including the domain and context), the formal model (specification), and finally the program itself. ${ }^{44}$ Lehman's early work on software engineering and the growth dynamics of programs started from the conviction that Software Evolution is intrinsic to large systems. During his long-standing

\footnotetext{
42 Stachowiak 1973

43 Lehman 1980

44 See also Floyd/Klischewski 1998.
}

\section{6}


work it became more and more clear that the concept of largeness could not provide the suitable basis for the study of Software Evolution. To solve this problem a new software classification scheme was proposed that was not based on size. Programs were divided into types S, P, and E, whereby the most important class in the present context are the E-type applications.

A program is called an S-type application, if the necessary and sufficient condition for the acceptance and proof of success of the whole development is its correctness in the full mathematical sense, relative to a given formal specification. It is assumed that the specification can be fully predetermined before the development of the software begins. The computation of mathematical functions and/or all sorts of formally definable transformations (e.g. compilers or proof procedures), are typical examples of S-type applications.

The E-type applications are closely related to the concept called Software Evolution. In contrast to the S-Type, E-programs are applications which become part of the domain they are modelling. That means that they are not only embedded into the real world but change the reality they are living in through their usage and activity. These programs become effective in reality, they modify what they model. Thus, E-Type programs are the ones we have to deal with in participatory media development.

Somehow halfway between S-type and E-type programs the P-type is located. These programs address problems that are fully specifiable, but the results of the execution has to be checked against the application domain and not its specification model. A typical example might be weather forecasting systems. They are mathematically correctly describable but their performance has to be demonstrated in reality, i.e. in comparison to the actual weather. On the other hand, the execution of the program does not change its domain, e.g. influence the weather, like E-type programs do.

\subsubsection{Software Evolution and some of its laws}

In its most general meaning the term evolution tries to capture the phenomenon of progressive change of the attributes of a system. From this point of view, not only nature evolves over time but also cities, societies, ideas, theories and also software. Of course, for all these different types of evolution we have to say what progress means in a particular field of evolution. The term evolution in software engineering is used to describe the cyclic and continual structure of maintenance, after the initial development phase. But it is important to mention that the meaning of maintenance in software engineering is incompatible with its common usage. Lehman and Fernández-Ramil point out that over the years it has been recognised that the term has to be carefully used in the context of software development. Normally, the term 
describes the efforts of people to soften or neutralise the processes of ageing, wear and tear or other forms of deterioration, which are typical and ongoing in every material artefact. For software maintenance it is more about the rectification of assumptions made about the application. We do not have to care about the ageing material but about the ageing of our thoughts, expectations, and models. "What happens with software is that it is changed or adapted to maintain it satisfactorily in changed domains and under new circumstances as judged by stakeholders such as users. Software is evolved to maintain embedded assumptions and its compatibility valid with respect to the world as it is now. Only in this sense, is the use of the term 'maintenance' appropriate in the software context." 45

The research on Software Evolution came up with a set of behaviour that is now known as Lehman's laws. Although these laws mainly apply to monolithic, proprietary software systems, some of the most important laws are obviously also valid for participatory design approaches or open source software developments. In the following, we do not present a complete list of all laws of Software Evolution but just a few that seem most appropriate within our participatory Unortkataster development: ${ }^{46}$

Continuing Change: An E-type program that is used must be continually adapted or else it becomes progressively less satisfactory;

Continuing Growth: Functional content of a program must be continually increased to maintain user satisfaction over its lifetime;

Declining Quality: E-type programs will be perceived as of declining quality unless rigorously maintained and adapted to a changing operational environment;

Feedback System: E-type programming processes constitute Multiloop, Multi-level Feedback systems and must be treated as such to be successfully modified or improved.

\section{Unortkataster: An urban experiment}

The development of the Unortkataster Köln is based on cooperation with a group of lead users. The working group Bild der Stadt of the initiative Leitbild 2020 formulated the idea of creating a technological platform for the citizens of Cologne to work on the city, from within the city. The continual talks with members of the community are the basis for an iterative software development process.

45 Lehman/Fernández-Ramil 2006

46 Lehman 1996

\section{8}


The Unortkataster application is an outcome of this collaboration and provides a community platform for the citizens of Cologne, based on a shared city-map. Adding state-of-the-art community functionalities to a digital map extends it to a multi-user interface with user administration capabilities and a database structure for storing media content. The map thereby allows people to mark areas or places on the map that are considered to be an Unort, and to add opinions and media content to justify the personal decision for the marking. What happens if maps become software interfaces? What does the term Unort suggest and how is the added content related to the meaning and function of the administrative Kataster maps?

On the Unortkataster, marking space becomes equal to starting and locating a discussion about that special location. The setting of a marker creates an Unort and is the start of a discussion about the place. Other people may, in succession, add comments or media content to express affirmation or disaffirmation. The Unortkataster is intended to facilitate processes, therefore time becomes the second principle of organisation. Since each action on the map is recorded by time, the development of the discussion can be regarded relative to space. It hence constitutes a spatio-temporal interface through which audiovisual communication is made possible. Conversations are connected with places, hence places become blogs. This allows subscribing to the information feed of a place and the tracking of the ongoing discussion. Places have a start and possible end in time. They may appear and vanish. If citizens decide to take part in the discussion about places in Cologne, the time layer of the map is updated and thereby the discussion is documented.

\subsection{Maps and software}

The originator of the General Semantics discipline, Alfred Korzybski stated, "A map is not the territory it represents, but if correct, it has a similar structure to the territory, which accounts for its usefulness." This points to what we have said about models and modelling. Our perception of reality is not reality itself but just a subjective version, or our own map that fulfils a certain function within a certain time interval and under the restriction of certain mental or actual operations.

Maps are figurative representations of relations or objects in the physical or logical world. They represent a certain selection that is related to the observation of an author. Maps contain hierarchies that influence how we see the world and are often based on arbitrary conventions. In the history of mapmaking, the setting of conventions was part of the legitimisation of power. If map users become mapmakers, they are empowered to set the conventions themselves and define their own territories. Software driven mapping sys- 
tems extend maps into dynamic structures. When maps become digital, they cut the line between the database and the image. The map becomes an interface that allows communication with the database. It thereby structures the circulation of information as an interaction of command, addressing, dating, storing and feedback.

Maps have to be upgraded constantly if they relate to a dynamic content. In the Unortkataster, the updating of the map is conceded to the participants. They start building the dynamic map upon the static map delivered by Google. Thus, the map's view evolves from a single-sided perspective to a many-sided perspective on the city. The significance of observation by users acquires another reputation.

\subsection{Orte and Unorte}

In order to analyse the phrasing of Unort, the meaning of Ort (place) is to be considered. Martina Löw states that places become visible as the target and result of the placing of social goods or people. Löw notes that places emerge by placements. In order to enable things to be placed, to be able to place something, space has to exist. On the other hand, the process of placing leads to spacing. The characteristics of places in physical space can be compared to those in an online environment: places appear by placings but are not identical with them. ${ }^{47}$ On an online map, a marked place may relate to a real place in the city but, at the same time, generate a different kind of place in the online setting. Marking an Unort in this context leads to another paradox: when people mark places on the Unortkataster-map this becomes an Unort but at the same time it is becoming an Ort. The indissoluble contradiction of the two terms is productive as it maintains controversy about the place - being an Unort or not - alive. The Unort may thus become a catalyst for social controversies about places in the city. It is thereby to be distinguished from the Non-Place that Marc Augé describes as a space where neither identities nor relations to history are legible. ${ }^{48}$

Marking on the application happens in a virtual environment. The question of which resulting changes may happen in physical space is, in contrast, related to administrative structures and political processes. But the Unortkataster delivers the possibility for a controversy in a setting where a single person is no longer authorised to change an environment, i.e. in public space. Public space, in this sense, is defined as the area that is exposed to the criticism of the public audience. It stands in opposition to private places and exclusive territories that allow an owner or a group of owners to exclude criti-

47 Löw 2001, pp. 198-200

48 Augé 1994

\section{0}



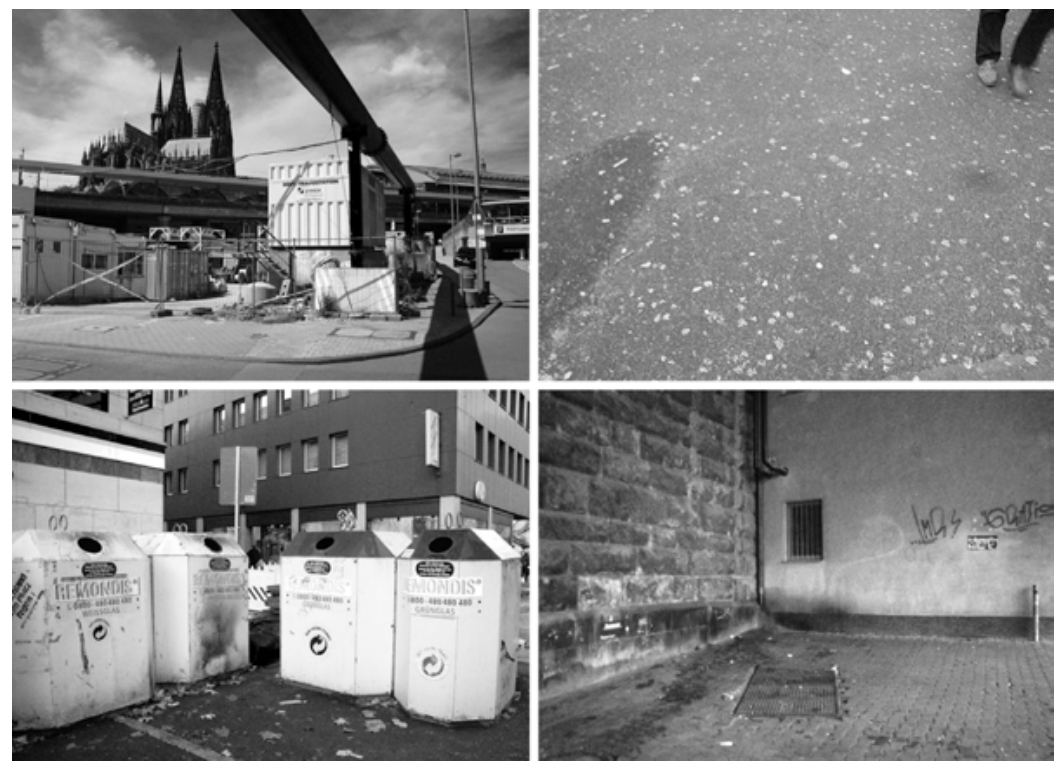

Fig. 2. Unorte in Cologne (Top left to bottom right): Building site close to the Dom, chewing gum, messy glass containers, dirty corner behind the station (Authors from top left to bottom right: Stefan Göllner, Keiko Takahashi, Jan Hopmann, Oliver Salkic)

cism to a certain degree. Thus, questions which are related to the relations of public and private properties become of major import for the platform.

Individual observation, marking places and the interlinking of different opinions, are the basic actions of participating in the Unortkataster. The tool thereby acts as a platform for organising this communication process. As the object of observation happens to be the public sphere, any favoured change will target the exponents of the public, which are represented firstly by the city administration. The Unortkataster thus provides insight into how citizens observe the city. The administration is excluded from this process but will be put in the position to follow the ongoing discussion and might draw conclusions out of this. Conflicts among users, property owners and city administration may arise because of activities on the platform. On that score, the setup of effective moderation mechanisms will be a crucial part of the research of the project. 


\subsection{Kataster leads to Unortkataster}

The administrative Kataster-map mainly intends to deliver an up-todate data basis for the land register and to protect private property rights. Although the Kataster is used for a completely different purpose than the Unortkataster, there are some crucial similarities between the two different kinds of maps. The Unortkataster does not target on archiving of facts and conditions. The content of the application therefore is soft: contributions and related media articulate opinions of participants that might be put into perspective by any kind of reply or addition of another user. The dynamics of an Unort lies in the impossibility of its final definition. The negative rating that distinguishes the Unort from the Ort refers to the personal opinion of its author and all conclusions drawn from it by related critics and supporters. The value of an Unort for the application is measured by the participants' interest in adding their own contributions. Both Kataster-maps have in common open-endedness and dependence on the permanent input of citizens. The Kataster documents evolution of private property and is thereby related to architecture and the built structure. The Unortkataster documents social dynamics that proceed in relation to the public city stage. When input stops, both applications become historic documents that demonstrate how the social sphere and built structure changed over time.

\subsection{Unortkataster as a web-probe}

Web 2.0 applications have recently shifted the focus away from the user and toward the community. With that shift, methods such as the persona that centre around the single user, its needs and its coupling to the interface, have become much less appropriate. Instead here, early probing implicitly developed towards a standard design method. Within this setting (and without explicit reference to the participatory design tradition), this methodology has been called "perpetual beta" ${ }^{49}$ : Applications are already opened for use during development, while their development cycle is possibly never declared finished: a praxis that, in the famous example of flickr, yielded a successful photo sharing application that had originally been developed (now abandoned) as an online game.

Methodologically, the Unortkataster developed through a crossover of practices described above. It started from a lead user approach while the Unortkataster itself may be seen as a Web 2.0 probe. The concept of the Unort was (and continues to be) discussed by probing the city through various media.

49 O’Reilly 2005

\section{2}


As an E-type application it borrows various ideas and methods from the history of software development, while at the same time neither a product nor a satisfied customer are its intended outcome. At best, it will generate discourse, structured by a dynamical (software based) map of the city, acting back upon the city and its real places through the discourse it generates. Similar to the

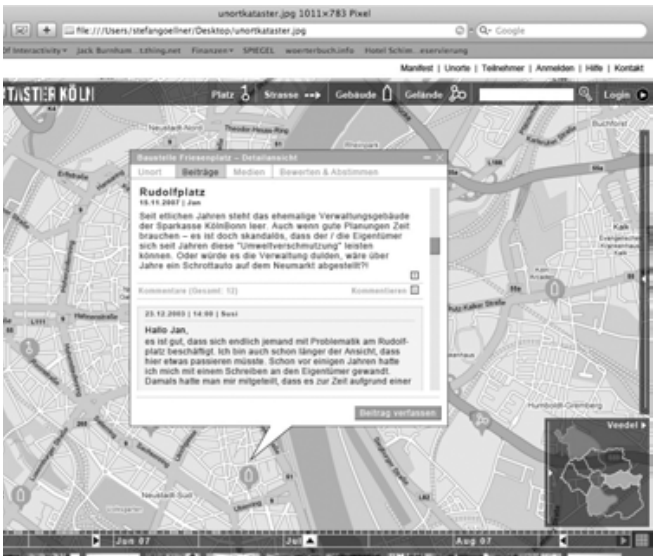

Fig. 3. Unortkataster application prototype developed together with lead users of the initiative »Leitbild Köln 2020 « prototypes of the participatory design tradition,

it is intended to create a common language for discussing the city seen as a space of shared problems. As a technology probe it is structuring this discourse, at the same time it is open to being shaped by it. Moreover, the database on which the Unortkataster is built may also have mobile access. How do Unorte change when they can be created and discussed in situ? How does perception of the city change, viewed through a mobile representation of the Unorte?

\section{Conclusion}

Participatory media development is not least about balances of power. In the end the forces and interests of the people driving the development of a certain application have to aid one another instead of blocking each other. An advised motivation, moderation and guidance approach seems to be the key to a successful collaborative development. Direction of the development and at the same time the balancing of power between the participants is subject to the new engineer-sociologist, it is actually his major power. Today we have to accept that there is no longer any technical activity that is not, at the same time, a cultural activity, and vice versa.

According to Wikipedia, collaborative governance is a process and a form of governance in which participants (parties, agencies, stakeholders) representing different interests are collectively empowered to make a policy deci- 
sion or make recommendations to a final decision-maker who will not substantially change consensus recommendations from the group. It is obvious that not all urban problems can be solved with participatory media on the basis of some sort of communication in the wild. For example, serious resource allocation conflicts (were does the money, manpower, engagement, etc. of the city go) also need intervention of a powerful government. Without that, the new forms of cooperation would mainly be "a new realm of creative expression and empowerment for the middle classes while the interests of the less powerful will continue to be represented (and distorted) by the devalued traditional welfare structures of (local) government."50

50 Maloutas/Malouta 2004

\section{4}




\section{References}

Augé, Marc (1994): Orte und Nicht-Orte der Stadt. Vorüberlegungen zu einer Ethnologie der Einsamkeit, Frankfurt a.M.: Fischer Verlag.

Baecker, Dirk (1994): »Soziale Hilfe als Funktionssystem der Gesellschaft«. Zeitschrift für Soziologie 23, 93-110.

Card, Stuart K./Newell, Allen/Moran, Thomas P. (1983): The Psychology of HumanComputer Interaction, Mahwah: Lawrence Erlbaum Associates, Inc.

Castells, Manuel (1998): The Information Age: Economy, Society and Culture, Vol. 3: End of Millennium, Massachusetts: Blackwell Publishers.

Cooper, Alan (1999): The Inmates Are Running the Asylum: Why High-tech Products Drive Us Crazy and How to Restore the Sanity, Indianapolis: Sams.

Denning, Peter/Dargan, Pamela (1996): »Action-Centered Design«. In: Terry Winograd (Ed.), Bringing Design to Software, Boston: Addison-Wesley, 105-120.

Fawcett-Tang, Roger/Owen, William (2002): Mapping. An illustrated guide to graphic navigational systems, Beverly: Rockport.

Floyd, C./Klischewski, R. (1998): »Modellierung - ein Handgriff zur Wirklichkeit. Zur sozialen Konstruktion und Wirksamkeit von Informatik-Modellen«. In: Klaus Pohl/Andy Schürr/Gottfried Vossen (Eds.), Modellierung '98 - Proceedings des GI-Workshops in Münster, 11.-13. März 1998 (= CEUR Workshop Proceedings 9), CEUR-WS.org, 21-26.

Gaver, William/Dunne, Anthony/Pacenti, Elena (1999): »Design: Cultural Probes«. New Visions of Human-Computer Interaction (= Interactions 6(1)), 21-29.

Graham, Steve/Marvin, Simon (1996): Telecommunications and the City, London/New York: Routlegde.

Hippel, Eric von (1986): »Lead Users. A Source of Novel Product Concepts«. Management Science 32, 791-805.

Hutchinson, Hilary/Mackay, Wendy/Westerlund, Bosse/Bederson, Benjamin B./Druin, Allison/Plaisant, Catherine/Beaudouin-Lafon, Michel/Conversy, Stéphane/Evans, Helen/Hansen, Heiko/Roussel, Nicolas/Eiderbäck, Björn/ Lindquist, Sinna/Sundblad, Yngve (2003): »Technology Probes: Inspiring Design for and with Families«. In: Gilbert Cockton/Panu Kohonen (Eds.), Proc. ACM Conference on Human Factors in Computing Systems, CHI 2003, (= CHI Letters 5(1)), 17-24.

Kuhn, Sarah (1996): „Design for People at Work«. In: Terry Winograd (Ed.), Bringing Design to Software, Boston: Addison-Wesley, 273-289.

Kuhn, Sarah/Winograd, Terry (1996): »Profile: Participatory Design«. In: Terry Winograd (Ed.), Bringing Design to Software, Boston: Addison-Wesley, 290-294.

Löw, Martina (2001): Raumsoziologie, Frankfurt a.M.: Suhrkamp.

Lehman, Meir M. (1980): »Programs, Life Cycles and Laws of Software Evolution«. In: Special Issue on Software Engineering (= Proc. IEEE 68(9)), 1060-1076. 
Lehman, Meir M. (1996): »Laws of Software Evolution Revisited«. In: Proceedings of the 5th European Workshop on Software Process Technology (= Lecture Notes in Computer Science 1149), London: Springer, 108-124.

Lehman, Meir M./Fernández-Ramil, Juan C. (2006): »Software Evolution«. In: Nazim H. Madhavji/Juan C. Fernández-Ramil/Dewayne E. Perry (Eds.), Software Evolution and Feedback: Theory and Practice, Chichester: John Wiley \& Sons, 7-40.

Luhmann, Niklas (1997): Die Gesellschaft der Gesellschaft, Frankfurt a.M.: Suhrkamp.

Lynch, Kevin (1960): The Image of the City, Cambridge: MIT Press.

Maloutas, Thomas/Pantelidou Malouta, Maro (2004): »The glass menagerie of urban governance and social cohesion: concepts and stakes/concepts as stakes«. International Journal of Urban and Regional Research 28(2), 449-465.

Mersch, Dieter (2000): »Erotik der Stadt. Das Problem von Urbanität zwischen Kommunikation und Ereignis«. In: Helmut Bott/Christoph Hubig/Franz Pesch/ Gerhart Schröder (Eds.), Stadt und Kommunikation im digitalen Zeitalter, Frankfurt a.M.: Campus, 189-209.

Muller, Michael J. (2002): »Participatory design: the third space in HCI«. In: Julie A. Jacko/Andrew Sears (Eds.), The human-computer interaction handbook: fundamentals, evolving technologies and emerging applications, Mahwah: Lawrence Erlbaum Associates, 1051-1068.

Mumford, Lewis (1966): Myth of the Machine, New York: Harcourt, Brace \& World.

Nake, Frieder (1989): »Das algorithmische Zeichen«. In: Kurt Bauknecht, Wilfried Brauer, Thomas A. Mück (Eds.), Informatik 2001 - Tagungsband der GI/OCG-Jahrestagung, Wien: Österr. Computer-Gesellschaft, 736-742.

O'Reilly, Tim (2005): „What is Web 2.0 - Design Patterns and Business Models for the Next Generation of Software«, O'Reilly Online. Online available: <http://www. oreillynet.com/pub/a/oreilly/tim/news/2005/09/30/what-is-web-20.html> (last access: October 2007).

Pias, Claus (2000): Computer Spiel Welten, Dissertation, Weimar: Bauhaus Universität.

Pias, Claus (2004): »Der Auftrag. Kybernetik und Revolution in Chile«. In: Daniel Gethmann/Markus Stauff (Eds.), Politiken der Medien, Zürich/Berlin: diaphanes, $131-154$.

Preece, Jennifer/Rogers, Yvonne/Sharp, Helen (2002): Interaction Design - Beyond Human-Computer Interaction, New York: Wiley.

Rönkkö, Kari (2005): »An empirical study demonstrating how different design constraints, project organization, and contexts limited the utility of personas«. In: Proceedings of the 38th Hawaii International Conference on System Sciences, Hawaii: IEEE.

Rötzer, Florian (1998): »Die Baustelle als Lebenswelt«. In: Franz Pröfener (Ed.), Zeitzeichen Baustelle, Frankfurt a.M./New York: Campus, 224-232.

Schroer, Markus (2006): Räume, Orte, Grenzen. Auf dem Weg zu einer Soziologie des Raums, Frankfurt a.M.: Suhrkamp.

\section{6}


Siebel, Walter (2004): Die europäische Stadt, Frankfurt a.M.: Suhrkamp.

Smith, Gillian C./Tabor, Phillip (1996): "The Role of the Artist-Designer«. In: Terry Winograd (Ed.), Bringing Design to Software, Boston: Addison-Wesley, 37-56.

Spinnuzi, Clay (2002): AScandinavian challenge, a US response: methodological assumptions in Scandinavian and US prototyping approaches«. In: Kathy Haramundanis / Michael Priestley (Eds.), Proceedings of the 20th Annual International Conference on Documentation, Toronto: ACM Press, 208-215.

Stachowiak, Herbert (1973): Allgemeine Modelltheorie, Wien: Springer.

Suchman, Lucy (1987): Plans and Situated Action, Cambridge: Cambridge University Press.

Wegner, Peter (1997): »Why interaction is more powerful than algorithms«. Communications of the ACM 40(5), 80-91.

Winograd, Terry/Flores, Fernando (1986): Understanding Computers and Cognition. A New Foundation for Design, Norwood: Ablex Publishing.

\section{Acknowledgements}

The Unortkataster is developed within the collaborative European research Project CITIZEN MEDIA, which is co-funded by the European Commission under the "Information Society Technologies" Sixth Framework Programme (Priority IST 2.41.6, Action Line: Networked Audio Visual Systems and Home Platforms). 


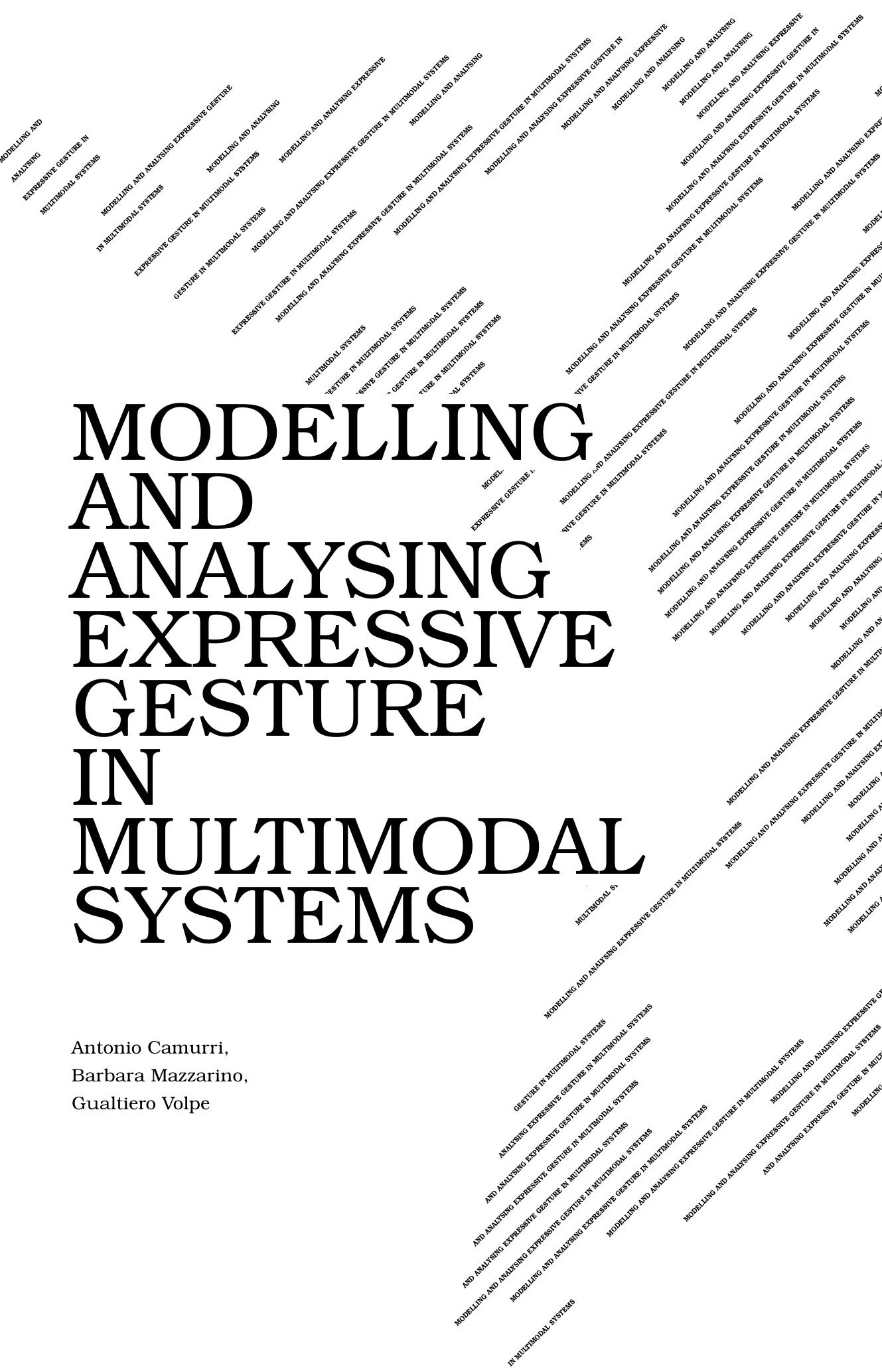




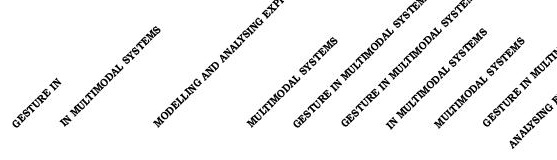

\section{Introduction}

This chapter presents research on the modelling of expressive gesture in multimodal interaction and on the development of multimodal interactive systems explicitly taking into account the role of expressive gesture in the communication process. In this perspective, a particular focus is on dance and music performances as first-class conveyors of expressive and emotional content.

Expressive gesture is a key concept in our research. ${ }^{1}$ This paper tries to deal with it, and introduces two experiments aiming at understanding the non-verbal mechanisms of expressive/emotional communication.

Several definitions of gesture exist in the literature. The most common use of the term is with respect to natural gesture, which is defined as a support to verbal communication. For Cassel and colleagues (1990) "[a] natural gesture means the types of gestures spontaneously generated by a person telling a story, speaking in public, or holding a conversation." McNeill (1992) in his well-known taxonomy divides the natural gestures generated during a discourse into four different categories: iconic, metaphoric, deictic, and beats. In a wider perspective, Kurtenbach and Hulteen (1990) define gesture as "a movement of the body that contains information."

In artistic contexts, and in particular in the field of performing arts, gestures are often not intended to denote things or to support speech as in the traditional framework of natural gesture, but the information they contain and convey is related to the affective/emotional domain. From this point of view, gestures can be considered "expressive" depending on the kind of information they convey: expressive gestures carry what Cowie et al. (2001) call "implicit messages" 3 , and what Hashimoto (1997) calls KANSEI. That is, they are responsible of the communication of a kind of information (what we call expressive content) which is different and in most cases independent from, even if often superimposed on, a possible denotative meaning, and which concerns aspects related to feelings, moods, affect, and emotional intentions.

For example, the same action can be performed in several ways, by stressing different qualities of movement: it is possible to recognise a person from the way she or he walks, but it is also possible to obtain information about the emotional state of a person by looking at her or his gait, e.g., if she or he is angry, sad, happy. In the case of gait analysis, we can therefore distinguish among several objectives and layers of analysis: a first one aiming at describ-

1 Camurri et al. 2005

2 A survey and a discussion of existing definition of gesture can be found in Cadoz and Wanderley 2000.

3 Cowie et al. 2001 
ing the physical features of the movement, for example in order to classify it; ${ }^{4}$ a second one aiming at extracting the expressive content gait conveys, e.g., in terms of information about the emotional state that the walker communicates through her or his way of walking. From this point of view, walking can be considered as an expressive gesture: even if no denotative meaning is associated with it, it still communicates information about the emotional state of the walker, i.e., it conveys a specific expressive content. In fact, in this perspective the walking action fully satisfies the conditions stated in the definition of gesture by Kurtenbach and Hulteen (1990): walking is "a movement of the body that contains information." Some studies can be found aiming at analysing the expressive intentions conveyed through everyday actions: for example, Pollick (2001) investigated the expressive content of actions like knocking or drinking.

If on the one hand expressive gestures partially include natural gestures, that is, natural gestures can also be expressive gestures, we face on the other hand a more general concept of expressive gesture that includes not only natural gestures but also musical, human movement, and visual (e.g. computeranimated) gestures. Our concept of expressive gesture is therefore somewhat broader than the concept of gesture as defined by Kurtenbach and Hulteen, since it also considers cases in which, with the aid of technology, communication of expressive content takes place even without an explicit movement of the body, or, at least, the movement of the body is only indirectly involved in the communication process. This can happen, for example, when using visual media. The expressive content is conveyed through a continuum of possible ways ranging from realistic to abstract images and effects: cinematography, cartoons, virtual environments with computer-animated characters and avatars, and expressive control of lights in the context of a theatre (e.g. related to actor's physical gestures). Consider, for example, a theatre performance: the director, choreographer, composer can ask actors, dancers, musicians, to communicate content through a number of expressive gestures (e.g., dance and/or music phrases). At the same time, technology allows the director to extend the language available to him. He can map motion or music features onto particular configurations of lights, in movements of virtual characters, in automatically generated computer music and live electronics. In this way, he can create an "extended" expressive gesture that, while still having the purpose of communicating an expressive content, is only partially related to explicit body movements: in a way, such "extended expressive gesture" is the result of a juxtaposition of several dance, music, and visual gestures, but it is not just the sum of them, since it also includes the artistic point of view

4 Quite a lot of research work can be found in the computer vision literature about gait analysis, see for example Liu et al. 2002.

\section{0}


of the director who created it, and it is perceived as multimodal stimuli by human spectators.

Our research on expressive gesture aims at the development of interactive multimedia systems enabling novel interaction paradigms and allowing a deeper engagement of the user by explicitly observing and processing his / her expressive gestures. Since artistic performances use non-verbal communication mechanisms to convey expressive content elaborately, we focused on performing arts, and in particular on dance and music performances, as a test-bed where computational models of expressive gesture and algorithms for expressive gesture processing can be developed, studied, and tested.

In particular, our attention has been focused on two aspects:

- Expressive gesture as a way to convey a particular emotion to the audience;

- Expressive gesture as a way to emotionally engage the audience.

Each of these has recently been the subject of experiments at our lab aiming at understanding which features in an expressive gesture are responsible for the communication of the expressive content, and how the dynamics of these features correlates with a specific expressive content.

In this paper, we concretely illustrate our approach by presenting two experiments focused on these two aspects.

The first one aims at (i) individuating which motion cues are mostly involved in conveying the dancer's expressive intentions (in term of basic emotions) to the audience during a dance performance and (ii) testing the models and algorithms developed by comparing their performances with spectators' ratings of the same dance fragments.

The second one investigates the mechanisms responsible for the audience's engagement in a musical performance. The aim of this experiment is again twofold: (i) individuating which auditory and visual cues are most involved in conveying the performer's expressive intentions and (ii) testing the developed model by comparing their performances with spectators' ratings of the same musical performances.

For the analysis of expressive gesture in these experiments a unifying conceptual framework was adopted. 


\section{The Layered Conceptual Framework}

The experiments presented in this paper address expressive gesture in music and dance performance.

While gesture in dance performance mainly concerns the visual/physical modality (even if the auditory components can be relevant as well), gesture in music performance uses both the auditory and the visual channels to convey expressive information, and, thus, it is multimodal in its essence. Gestures in music performance are not only the expressive and functional gestures that a performer physically makes, but also include expressive gestures present in the sound produced. When we define gestures as structural units that have internal consistency and are distinguished in time and quality from neighbouring units, it is possible to analyse gestures in both modalities. Multimodality is therefore a key issue. In order to deal with multimodal input a unifying conceptual framework has been adopted. ${ }^{5}$ It is based on a layered approach ranging from low-level physical measures (e.g., position, speed, acceleration of body parts for dance gestures, sampled audio signals or MIDI messages for music gesture) toward descriptors of overall gesture features (e.g., motion fluency, directness, impulsiveness for dance gestures, analysis of melodic and harmonic qualities of a music phrase for music gestures).

This layered approach is sketched in Figure 1. Each layer is depicted with its inputs, its outputs, and the kind of processing it is responsible for. In the following sections, each layer will be discussed with respect to its role in the two experiments.

Our conceptual framework, here presented for analysis, can also be applied for synthesis of expressive gesture: for example for the generation and control of the movement of avatars, virtual characters, or robots in Mixed Reality scenarios, as well as for the synthesis and interpretation of music. Examples of synthesis of expressive movement and expressive audio content are well documented in literature. ${ }^{6}$

Finally, it should be noticed that in the perspective of developing novel interactive multimedia systems for artistic applications, such a framework should be considered in the context of a broader Mixed Reality scenario in which virtual subjects (e.g., virtual characters) who behave both as observers and as agents perform the four layers of processing in the analysis of observed expressive gestures and in the synthesis of expressive gestures to communicate (directly or remotely) with other real and virtual subjects.

$5 \quad$ Camurri et al. 2005

6 See e.g. the EMOTE system (Chi et al. 2000) for generation of movement of avatars and virtual characters based on high level motion qualities, and the systems for synthesis of expressive music performances developed at KTH (Friberg et al. 2000) and by the DEI-CSC group at the University of Padova (Canazza et al. 2000).

\section{2}


High-level expressive information: (Experiment 1) Recognised emotions (e.g., anger, fear, grief, joy); (Experiment 2) Predict spectators' intensity of emotional experience.

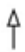

\section{Layer 4 - Concepts and structures}

Modelling techniques (for example, classification in terms of basic emotions, or prediction of intense emotional experience in spectators): e.g., based on multiple regression, neural networks, support vector machines, decision trees, Bayesian networks.

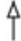

Segmented gestures and related parameters (e.g., absolute and relative duratons), trajectories representing gestures in semantic spaces.

$\Delta$

\section{Layer 3 - Mid-level features and maps}

Techniques for gesture segmentation: motion segmentation (e.g., in pause and motion phases), segmentation of musical excerpts in musical phrases. Representation of gestures as trajectories in semantic spaces (e.g., Laban's Effort space, energy-articulation space)

\section{个}

Motion and audio descriptors: e.g., amount of energy - loudness, amount of contraction/expansion spectral width and melodic contour, low fluency - roughness etc.

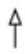

\section{Layer 2 - Low-level features}

Computer vision techniques on the incoming images, statistical measures, signal processing techniques on audio signals.

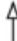

- Images pre-processed to detect movement, trajectory of points (e.g., trajectories of body parts, trajectories of dancers in the space)

- MIDI and audio pre-processed to detect spectral and temporal low-level features.

$\stackrel{\wedge}{\uparrow}$

\section{Layer 1 - Physical signals}

Analysis of video and audio signals: techniques for background subtraction, motion detection, motion tracking (e.g., techniques for colour tracking, optical flow based feature tracking), techniques for audio pre-processing and filtering, signal conditioning.

Data from several kinds of sensors, e.g., images from video cameras, positions from localisation systems, data from accelerometers, sampled audio, MIDI messages.

Fig. 1. The layered conceptual framework and its instantiation in the two experiments 


\section{Modeling expressive gesture in dancers}

As an example of analysis of expressive gesture in dance performance, we discuss an experiment carried out in collaboration with the Department of Psychology of the University of Uppsala (Sweden) in the EU-IST MEGA project.

The aim of the experiment was twofold: (i) individuating which motion cues are mostly involved in conveying the dancer's expressive intentions to the audience during a dance performance and (ii) testing the developed models and algorithms by comparing their performances with spectators' ratings of the same dance fragments.

In the case of this experiment, expressive gesture was analysed with respect to its ability to convey emotions to the audience. The study focused on the communication through dance gesture and recognition by spectators of the four basic emotions: anger, fear, grief, and joy.

The research hypotheses are grounded in the role of the Laban's dimensions in dance gesture, as described in Laban's Theory of Effort: ${ }^{7}$

- The time dimension in terms of overall duration of time and tempo changes also elaborated as the underlying structure of rhythm and flow of the movement;

- The space dimension in its aspects related to Laban's "personal space" e.g., to what extent limbs are contracted or expanded in relation to the body centre;

- The flow dimension in terms of analysis of shapes of speed and energy curves, and frequency/rhythm of motion and pause phases;

- The weight dimension in terms of amount of tension and dynamics in movement, e.g., vertical component of acceleration.

These cues were predicted to be associated in different combinations to each emotion category. ${ }^{8}$

\subsection{The experiment}

An experienced choreographer was asked to design a choreography such that it excluded any propositional gesture or posture and it avoided stereotyped emotions.

7 Laban 1963; Laban/Lawrence 1947

8 Details can be found in Camurri/Lagerlöf/Volpe 2003.

\section{4}


In Uppsala, five dancers performed this same dance with four different emotional expressions: anger, fear, grief and joy. Each dancer performed all four emotions. The dance performances were video-recorded by two digital videocameras (DV recording format) standing fixed in the same frontal view of the dance (a spectator view). One camera obtained recordings to be used as stimuli for spectators' ratings. The second video camera was placed in the same position but with specific recording conditions and hardware settings to simplify and optimise automated recognition of movement cues (e.g., high speed shutter). Dancers' clothes were similar (dark), contrasting with the white background, in an empty performance space without any scenery. Digitised fading eliminated facial information and the dancers appeared as dark and distant figures against a white background.

The psychologists in Uppsala then proceeded in collecting spectators' ratings: the dances were judged with regard to perceived emotion by 32 observers, divided in two groups. In one group ratings were collected by 'forced choice' (choose one emotion category and rate its intensity) for each performance, while the other group was instructed to use a multiple choice schema, i.e., to rate the intensity of each emotion on all four emotion scales for each performance.

At the same time, at the InfoMus Lab we proceeded in extracting motion cues from the video recordings and in developing models for automatic classification of dance gestures in terms of the basic emotion conveyed.

\subsection{Automated Extraction of Motion Cues}

Extraction of motion cues followed the conceptual framework described in Section 2.

\subsubsection{Layer 1}

In the case of analysis of dance performance from video, layer 1 is responsible for the processing of the incoming video frames in order to detect and obtain information about the motion that is actually occurring. It receives as input images from one or more videocameras and, if available, information from other sensors (e.g., accelerometers). Two types of output are generated: processed images and trajectories of body parts. Layer 1 accomplishes its task by means of consolidated computer vision techniques usually employed for real-time analysis and recognition of human motion and activity. ${ }^{9}$ It

9 See for example the temporal templates technique for representation and recognition of human movement described in Bobick/Davis 2001. 
should be noted that in contrast to the research of Bobick and J. Davis, we do not aim at detecting or recognising a specific kind of motion or activity. The techniques we use include feature-tracking based on the Lucas-Kanade algorithm ${ }^{10}$, skin colour tracking to extract positions and trajectories of hands and head, an algorithm to divide a body silhouette into sub-regions, and Silhouette Motion Images (SMIs). A SMI is an image carrying information about variations of the silhouette shape and position in the last few frames. SMIs are inspired by motion-energy images (MEI) and motion-history images (MHI). ${ }^{11}$ They differ from MEIs in the fact that the silhouette in the last (more recent) frame is removed from the output image: in such a way only motion is considered, while the current posture is skipped. Thus, SMIs can be considered as carrying information about the "amount of motion" which has occurred in the last frames. Information about time is implicit in SMI and is not explicitly recorded. We also use an extension of SMIs, which takes into account the internal motion in silhouettes. In such a way we are able to distinguish between global movements of the whole body in the General Space and internal movements of body limbs inside the Kinesphere.

\subsubsection{Layer 2}

Layer 2 is responsible for the extraction of a set of motion cues from the data coming from low-level motion tracking. Its inputs are the processed images and the trajectories of points (motion trajectories) coming from Layer 1. Its output is a collection of motion cues describing movement and its qualities. According to the research hypotheses described above, the cues extracted for this experiment include:

- Cues related to the amount of movement (energy) and in particular what we call Quantity of Motion (QoM). GoM is computed as the area (i.e., number of pixels) of an SMI. ${ }^{12}$ It can be considered as an overall measure of the amount of detected motion, involving velocity and force;

- Cues related to body contraction/expansion, and in particular the Contraction Index (CI). CI is a measure, ranging from 0 to 1 , of how the dancer's body uses the space surrounding it. The algorithm to compute the $\mathrm{CI}^{13}$ combines two different techniques: the individuation of an ellipse approximating the body silhouette and computations based

10 Lucas/Kanade 1981

11 Bradsky/Davis 2002; Bobick/Davis 2001

12 Camurri/Lagerlöf/Volpe 2003

13 Camurri/Lagerlöf/Volpe 2003

\section{6}


on the bounding region. The former is based on an analogy between the image moments and mechanical moments: ${ }^{14}$ the eccentricity of the approximating ellipse is related to body contraction/expansion. The latter compares the area covered by the minimum rectangle surrounding the dancer with the area currently covered by the silhouette;

- Cues derived from psychological studies ${ }^{15}$ such as amount of upward movement, dynamics of the Contraction Index (i.e., how much CI was over a given threshold along a time unit);

- Cues related to the use of space: length and overall direction of motion trajectories;

- Kinematical cues (e.g., velocity and acceleration) calculated on motion trajectories.

For those cues depending on motion trajectories a Lucas-Kanade feature tracker was employed in Layer 1. A redundant set of 40 points randomly distributed on the whole body was tracked. Points were reassigned each time dancers stopped their motion (i.e., a pause was detected) so that a small and non-significant amount of points was lost during tracking. Overall motion cues were calculated by averaging the values obtained for each trajectory.

\subsubsection{Layer 3}

Layer 3 is in charge of segmenting motion in order to individuate motion and non-motion (pause) phases. QoM was used to perform such segmentation. GoM is related to the overall amount of motion and its evolution in time can be seen as a sequence of bell-shaped curves (motion bells). In order to segment motion, a list of these motion bells was extracted and their features (e.g., peak value and duration) computed. An empirical threshold was defined for these experiments: the dancer is considered to be moving if its current QoM is above $2.5 \%$ of the average value of the $\mathrm{BoM}$ computed along each whole dance fragment.

Segmentation allows further higher-level cues to be extracted, e.g., cues related to the time duration of motion and pause phases. A concrete example is the Directness Index (DI), calculated as the ratio between the length of the straight trajectory connecting the first and the last point of a motion trajectory and the sum of the lengths of each segment constituting the trajectory. Moreover, segmentation can be considered as a first step toward the analysis of the rhythmic aspects of the dance. Analysis of the sequence of pause and motion phases and their relative time durations can lead to a first evaluation

14 Kilian 2001

15 See for example Boone/Cunningham 1998 
of dance tempo and its evolution in time, i.e., tempo changes and articulation (the analogue to music legato/staccato). Parameters from pause phases can also be extracted to differentiate real standing-still positions from active pauses involving low-motion (hesitation, subtle swaying or tremble, e.g., due to instable equilibrium or fatigue).

Furthermore, motion fluency and impulsiveness can be evaluated. They are related to Laban's Flow and Time axes. Fluency can be estimated starting from an analysis of the temporal sequence of motion bells. A dance fragment performed with frequent stops and restarts (i.e., characterised by a high number of short pause and motion phases) will gain the result of being less fluent than the same movement performed in a continuous, "harmonic" way (i.e., with a few long motion phases). The hesitating, bounded performance will be characterised by a higher percentage of acceleration and deceleration in the time unit (due to the frequent stops and restarts), a parameter that has been demonstrated to be of relevant importance in motion flow evaluation. ${ }^{16}$,

A first measure of impulsiveness can be obtained from the shape of a motion bell. In fact, since $\mathrm{Q}$ oM is directly related to the amount of movement detected, a short motion bell having a high peak value will be the result of an impulsive movement (i.e., a movement in which speed rapidly moves from a value near or equal to zero, to a peak and back to zero). On the other hand, a sustained, continuous movement will show a motion bell characterised by a relatively long time period in which the $\mathrm{BoM}$ values have little fluctuations around the average value (i.e., the speed is more or less constant during the movement).

Fluency and impulsiveness are also related to the spectral content of the QoM: a movement having significant energy at high frequencies is a candidate to be characterised by low fluency.

\subsubsection{Layer 4}

In this experiment, Layer 4 collects inputs from Layers 2 and 3 (18 variables have been calculated on each detected motion phase) and tries to classify a motion phase in terms of the four basic emotions anger, fear, grief and joy.

As a first step, statistical techniques have been used for a preliminary analysis: descriptive statistics and a one-way ANOVA have been computed for each motion cue. ${ }^{17}$

16 See for example Zhao 2001, where a neural network is used to evaluate Laban's flow dimension.

17 Results of such preliminary analysis can be found in Mazzarino 2002; Camurri/ Lagerlöf/Volpe 2003; Volpe 2003.

\section{8}


Decision tree models were then built for classification. Five training sets ( $85 \%$ of the available data) and five test sets (15\% of the available data) were extracted from the data set. The samples for the test sets were uniformly distributed along the four classes and the five dancers. Five decision trees were built on the five training sets and evaluated on the five test sets. The Gini's index of heterogeneity was used for building the decision trees. Decision trees were selected for this study since they produce rules that can be used to interpret the results. Comparison with other classification techniques (e.g., Neural Networks, Support Vector Machines) remains a task for possible future work.

The above-described techniques in the four layers were implemented in our EyesWeb open software platform. ${ }^{18}$ The Expressive Gesture Processing Library ${ }^{19}$ includes these and other processing modules.

\subsection{Results}

Results from spectators' ratings are described in Camurri/Lagerlöf/Volpe 2003. The results obtained on the five decision trees can be summarised as follows (results for the best model are reported in Tables 1 and 2 (see p. 232) showing the confusion matrices for the training set and for the test set respectively).

Two models ( 3 and 5 ) fit the data set quite well; the rates of correct classification on the training set for these two models averaged over the four classes are $78.5 \%$ and $61.6 \%$, respectively. Three models $(1,2$, and 4$)$ have difficulties in classifying fear. The rates of correct classification on the training set for these three models averaged over the four classes are $41.9 \%, 38.7 \%$, and $36.0 \%$, respectively. Models 2 and 4 also have problems with joy, which means that they distinguish correctly only between anger and grief.

A similar situation can be observed in the evaluation carried out on the test set: only models 3 and 5 are able to classify all four emotions correctly. Model 1 cannot classify fear, while models 2 and 4 cannot classify fear and joy.

The rates of correct classification on the test set for the five models averaged on the four classes are respectively: $40 \%, 36 \%, 36 \%, 26 \%$, and $40 \%$. Thus the average rate of correct classification on the five models is $35.6 \%$. Except for model 4, they are all above chance level (25\%). Model 5 can be considered as the best model, since it has a rate of correct classification of $40 \%$ and is able to classify all four emotions.

18 Camurri et al. 2000; Free download of technical documentation and full software environment are available at <http: / www.eyesweb.org>.

19 Camurri/Mazzarino/Volpe 2003

\section{9}




\begin{tabular}{|c|c|c|c|c|c|c|c|}
\hline Class & Total & \%Correct & \%Error & Anger & Fear & Grief & Joy \\
\hline Anger & 64 & 71.9 & 28.1 & 46 & 10 & 2 & 6 \\
\hline Fear & 60 & 61.7 & 38.3 & 15 & 37 & 1 & 7 \\
\hline Grief & 86 & 47.7 & 52.3 & 10 & 19 & 41 & 16 \\
\hline Joy & 74 & 64.9 & 35.1 & 13 & 8 & 5 & 48 \\
\hline
\end{tabular}

Table 1. Confusion matrix for the training set for the best decision tree

\begin{tabular}{|c|c|c|c|c|c|c|c|}
\hline Class & Total & \%Correct & \%Error & Anger & Fear & Grief & Joy \\
\hline Anger & 12 & 41.7 & 58.3 & 5 & 3 & 0 & 4 \\
\hline Fear & 13 & 30.8 & 69.2 & 6 & 4 & 2 & 1 \\
\hline Grief & 12 & 41.7 & 58.3 & 2 & 0 & 5 & 5 \\
\hline Joy & 13 & 46.1 & 53.8 & 4 & 0 & 3 & 6 \\
\hline
\end{tabular}

Table 2. Confusion matrix for the test set for the best decision tree

These rates of correct classification, which at first glance seem to be quite low ( $40 \%$ being the best model), should however be considered with respect to the rates of correct classification by spectators who were asked to classify the same dances. In fact, spectators' ratings collected by psychologists in Uppsala show a rate of correct classification (averaged over the 20 dances) of $56 \%$.

The rate of correct automatic classification (35.6\%) is thus in between chance level (25\%) and the rate of correct classification for human observers (56\%).

Furthermore, if the rate of correct classification for human observers is considered as a reference, and percentages are recalculated taking it as $100 \%$ (i.e., relative instead of absolute rates are computed), the average rate of correct automatic classification with respect to spectators is $63.6 \%$, and the best model (i.e., model 5) obtains a rate of correct classification of $71.4 \%$.

By observing the confusion matrix of the best model (both for the test set and for the training set) it can be noticed that fear is often classified as anger. This particularly holds for the test set, where fear is the basic emotion which receives the lowest rate of correct classification, since 6 of the 13 motion phases extracted from fear performances are classified as anger. Something similar can be observed in spectators' ratings. ${ }^{20}$

20 Camurri/Lagerlöf/Volpe 2003 
A deeper comparison with spectator's ratings shows that while anger is generally well classified both by spectators and by the automatic system (60\% for automatic recognition vs. $60.6 \%$ for spectators), quite bad results are obtained for fear (below chance level for the automatic classification). The biggest overall difference between spectators and automatic classification was observed for joy $(70.4 \%$ for spectators vs. $27.7 \%$, just above chance level, for automatic classification). In the case of grief instead, automatic classification performs better than human observers $(48.3 \%$ for automatic classification vs. $39.8 \%$ for spectators): this happens in five cases and mainly for grief. In seven cases, the rate of correct classification for the automatic system is below chance level (and this always happens for fear). In one case, automatic classification did not succeed in finding the correct emotion (Fear - Dancer 4), but spectators obtained $67 \%$ of correct classification. In another case, spectators' ratings are below chance level (Grief - Dancer 5), but automatic classification could obtain a rate of correct classification up to $50 \%$.

Dancer 1 obtained the lowest rates of correct classification both from spectators and from the models. Dancer 5 obtains similar rates from both. Dancer 2 is the best classified by spectators and also obtains a quite high rate (with respect to the other dancers) in automatic classification.

\section{Analysis of expressive gesture in music performance}

The second experiment investigates the mechanisms responsible for the audience's engagement in a musical performance. ${ }^{21}$ The aim of this experiment is again twofold: (i) individuating which auditory and visual cues are most involved in conveying the performer's expressive intentions and (ii) testing the model developed by comparing its performance to spectators' ratings of the same musical performances.

In this experiment, expressive gesture was analysed with respect to its ability to convey the intensity of emotion to the audience. The study focused on communication through visual and auditory performance gestures of emotional intensity and the effect of it on spectators' emotional engagement.

The research hypotheses combine hypotheses from Laban's Theory of Effor $^{22}$ with hypotheses stemming from performance research ${ }^{23}$ and research on the intensity of emotion and tension in music and dance: ${ }^{24}$

21 More detailed description of such an experiment is available from Timmers et al. 2006.

22 Laban 1947, Laban/Lawrence 1963

23 Palmer 1997; Timmers 2002

24 Krumhansl 1996; Krumhansl/Schenck 1997 
1. Emotional intensity is reflected in the degree of openness (release) or contraction (tension) of the torso of the performer.

2. Emotional intensity is communicated by the main expressive means for a pianist: tempo and dynamics.

3. Intensity increases and decreases with energy level (speed of movements, loudness, tempo).

4. Intensity is related to the performer's phrasing: it increases towards the end of the phrase and decreases at the phrase boundary with the introduction of new material.

\subsection{Method}

\subsubsection{Musical performance}

A professional pianist was asked to perform an emotionally engaging piece of his choice at a concert that was organised for the experiment's purpose. He performed the piece first without public in a normal manner and an exaggerated manner and then with public in a normal, concert manner. Exaggerated meant with enhanced expressivity, which was, according to the pianist, consistent with the style of performance of the early 20th Century.

He performed on a Yamaha Disklavier, which made it possible to register MIDI information of the performance. In addition, audio recordings were made, and video recordings from four sides (Fig. 2). The video recordings from the left were presented to the participants of the experiment.

The pianist chose to perform Etude Op. 8 no. 11 by Alexander Skriabin, which is a slow and lyrical piece (Andante cantabile) in a late Romantic style that has a considerable amount of modulations. According to the pianist, the piece can be played with a lot of freedom. Theoretically, the piece has a simple A B A with coda structure (A A' B A" A"' C, to be more precise), but the pianist interpreted the line of the music differently: the first main target of the music is a release of tension halfway through the B section. Everything preceding this target point is a preparation for this tension release. The A section is anyway preparatory; it leads towards the start of the B section, which is the real beginning of the piece. After this release of tension, the music builds up towards the dramatic return of the theme of the A section. This prepares for the second possible point of tension release halfway through the coda at a general pause. The release, however, is not continued, and the piece ends most sadly.

\section{2}



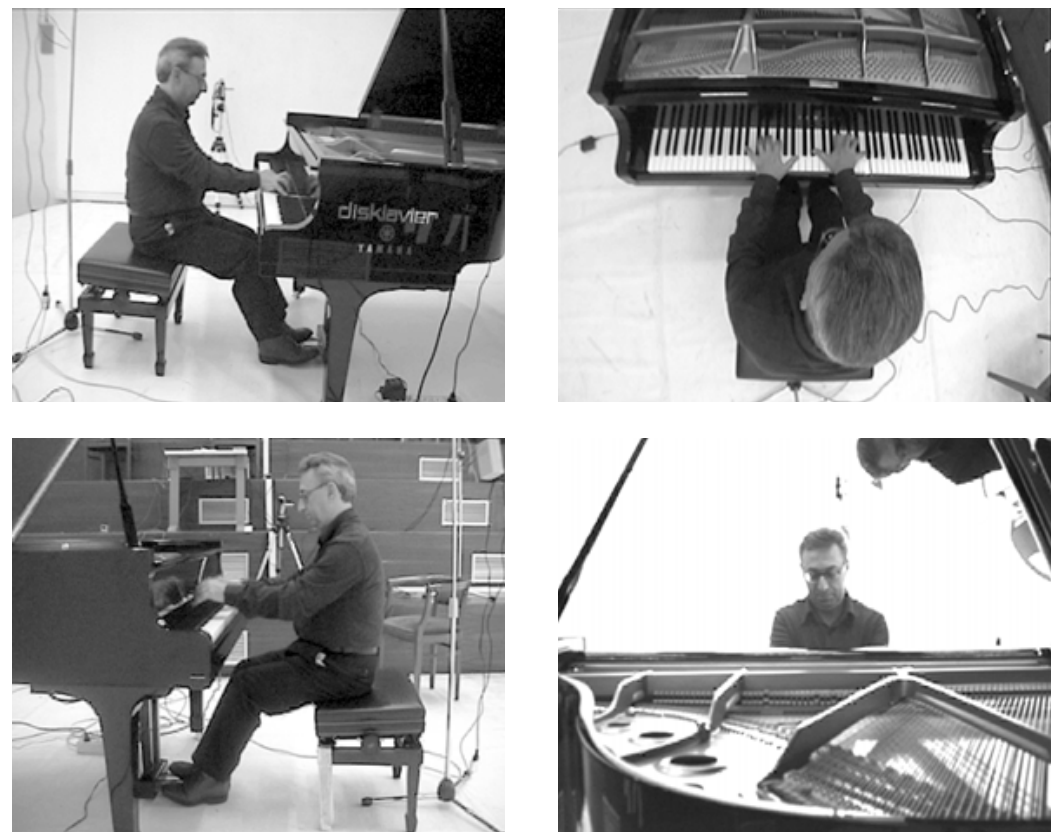

Fig. 2. Video recordings of the piano performances (right, top, left, and front views)

\subsubsection{Participants}

12 people participated in the experiment; among them were four musicians. The participants varied greatly in musical experience. Some of them never had had music lessons and hardly listened to classical music, while others had basically performed classical music for their entire lives.

\subsubsection{Procedure}

The participants saw the performances on a computer screen and heard them on loudspeakers. They saw and heard the performances two times. During the first hearing, they indicated the phrase boundaries in the music by pressing the button of the joystick. During the second hearing, they indicated to what extent they were emotionally engaged with the music by moving a MIDI-slider up and down. The order of the repeated performances was randomised over participants. The whole procedure was explained to them by a written instruction and a practice trial. 


\subsection{Analyses}

\subsubsection{Auditory Performance Data}

The key velocity and onset times of notes were extracted from the MIDI files (layer 1). From this, the average key velocity for each quarter note was calculated as well as inter-onset intervals (IOI's) between successive quarter notes (layer 2). The quarter note IOI is an accurate measure of local duration, while key velocity corresponds well to local loudness. These measures were interpreted as a direct expression of emotional intensity and as an expression of musical phrasing. ${ }^{25}$

\subsubsection{Visual Performance Data}

For the analysis of the movement of the pianist, we concentrated on the movement of the head, which shows both backward-forward movement (y-direction) and left-right movement (x-direction). The position of the head was measured, using the Lucas and Kanade feature-tracking algorithm ${ }^{26}$ that assigns and tracks a specified number (in our case 40) of randomly assigned moving points within a region (layer 1). Velocity and acceleration were calculated for each trajectory using the symmetric backward technique for the numeric derivative (layer 2). Average values of position and velocity among the forty trajectories were calculated for both the $\mathrm{x}$ and $\mathrm{y}$ component. In addition, the velocity values were integrated for the $\mathrm{x}$ and $\mathrm{y}$ movement to get a general measure of amount of movement over time. Redundancy in the number of points (i.e., forty points instead, for example, of just the barycentre of the blob) allowed us to get more robust and reliable values for velocity. A low-pass filter was applied to smooth the data obtained. Measures were summarised per quarter-note in order to be comparable to the other measures.

\subsubsection{Spectators' ratings}

For each quarter note in the performance, the number of people who indicated a phrase boundary was calculated by summing the number of boundary indications per quarter note over participants. This sum per quarter note was expressed as a multiple of chance-level, where chance-level corresponded to an even distribution of the total of segment-indications over quarter notes. This segmentation measure will be abbreviated as SM.

25 See 4.3 Results.

26 Lucas/Kanade 1981

\section{4}


The indication of emotional engagement was measured at a sampling rate of $10 \mathrm{~Hz}$ using a MIDI-slider that had a range from 0 to 127 . The average level of the MIDI-slider (emotion measure, abbreviation EM) per quarter note was calculated for each participant separately.

An EyesWeb patch application was developed to store and process participants' data in real-time.

\subsection{Results}

\subsubsection{Auditory Performance Data (layer 3)}

The resulting profiles of quarter note key velocity and quarter note IOI were highly similar for the three performances: they all started at a slow tempo and with soft dynamics, and had considerable crescendi and accelerandi in the A section, a diminuendo and crescendo in the B section accompanied by first a highly variable tempo and thereafter an accelerando, a fast and loud return of the A section with limited variation in tempo and dynamics, a soft and slower repeat of the theme, and a coda that fades away in dynam-

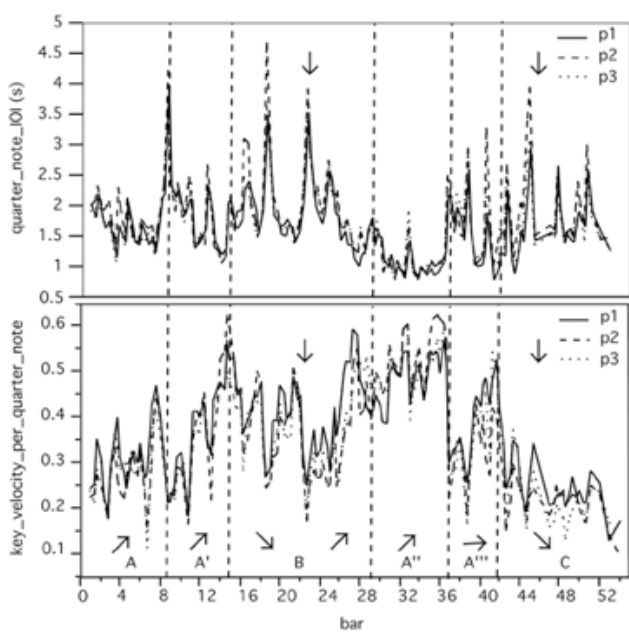

Fig. 3. The duration per quarter note and the key velocity per quarter note as it varies throughout the Skriabin Etude. Separate plots for the three performances of the piece. Vertical lines indicate section boundaries. Arrows are explained in the text. ics and tempo (Fig. 3). This global pattern is indicated by arrows at the bottom of Figure 3.

In addition to this global pattern, the IOI profile shows the characteristic peaks of phrase-final lengthenings. It shows this at a fairly high density and large magnitude, except in the forte return of the A section (A"). The key velocity profile shows drops in velocity at most phrase boundaries, but these are compensated by strong crescendi in most sections. 


\subsubsection{Visual Performance Data (layer 3)}

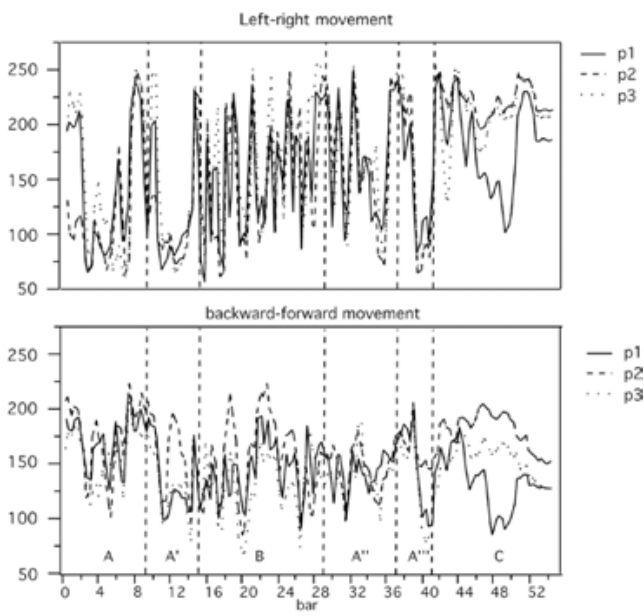

Fig. 4. The position of the head plotted per quarter note. Upper panel shows left-right position ( $x$ ) and bottom panel the backward-forward position (y). Separate plots for the three performances of the piece. Vertical lines indicate section boundaries.
The position of the head is plotted in Figure 4 for two dimensions: leftright (upper panel) and backward-forward (bottom panel). The movement of the head was especially pronounced and especially consistent over performances in the left-right direction (correlation between $\mathrm{p} 1$ and p2 and between p2 and p3 was 0.79 ; it was 0.83 between $\mathrm{p} 1$ and $\mathrm{p} 3$ ). The backward-forward movement becomes more pronounced for the later performances (p2 and p3). The periodic movement is relatively fast in the mid-

dle parts of the piece (B and A'"') and slower in the outer parts. This suggests an intensification towards the middle followed by a relaxation towards the end.

\subsubsection{Relation between performance data}

Correlations between performance measures were calculated to check the coherence between measures. Key velocity and IOI are negatively correlated ( $\mathrm{r}=-0.51$ on average). Velocity of head movement is positively correlated with key velocity ( $r=0.45$ on average) and negatively with IOI $(r=-0.25$ on average). The low correlation between values is partly due to the asynchrony between the periodicity of the measures. If peak values (maximum for key and movement velocity and minimum for IOI) per two bars are correlated, agreement between movement and sound measures becomes higher. Especially the two velocity measures turn out to be highly correlated $(r=0.79$ on average for key and movement velocity, versus $r=-0.38$ on average for movement velocity and IOI). 
All performance measures show a periodic increase and decrease. To check the relation between these periodicities and the musical structure, the location of mimima in key velocity, and maxima in IOI, x-position and $y$-position were compared to the location of phrase boundaries. Generally, the Skriabin Etude has a local structure of two-bar phrases. The forward and the left position of the performer were taken as start/end point for periodicity. IOI was most systematically related to the two-bar phrasing of the Skriabin piece, followed by key velocity. 55\% of the phrase-boundaries were joined by a slowing-down in tempo. The other phrase boundaries were directly followed by a slowing down in tempo (a delay of 1 quarter note). For the key velocity, $42 \%$ of the phrase-boundaries coincided with a minimum in key velocity, $15 \%$ were anticipated by a minimum and $28 \%$ followed by a minimum. The period boundaries of the movement of the pianist hardly synchronised with the score-phrasing. The location of these boundaries varied greatly with respect to the two-bar score-phrasing.

\subsubsection{Relation between Performance and Listeners Data (layer 4)}

In this study, we had four hypotheses concerning the communication of intensity of emotion in musical performances.

Hypothesis 1 predicts that intensity of emotion is positively correlated with backward-forward movement (y). This hypothesis is easily tested and contradicted: the correlation between listeners' indication of intensity of emotion and backward-forward position is negative ( $\mathrm{r}$ is $-0.23,-0.50,-0.29$ for $\mathrm{p} 1$, p2 and p3, respectively). It is also contradicted with respect to the other performance data: $y$-position is negatively correlated with velocity and positively correlated with IOI, which means that the performer moves forward in soft and slow passages and backwards in louder and faster passages.

Hypothesis 2 predicts that tempo and dynamics cooperate to communicate intensity of emotion. This is made problematic by the fairly low correlation between IOI and key velocity and by their different relation with the score-phrasing. Instead the performance data suggests a differentiation in function between the two expressive means, and tempo strongly communicates phrasing.

Hypothesis 3 predicts high movement to correspond with intense dynamics and fast tempi. As we have seen in the previous section, dynamics and movement velocity agree more strongly than movement velocity and tempo. Especially the variation in velocity peaks corresponds.

Hypothesis 4 relates phrasing to intensity of emotion. A clear phrase ending is predicted to coincide with a release in emotional intensity. 
A series of multiple regression analyses was carried out. In the first analysis, quarter note IOI, key velocity, and movement velocity were used to predict EM. In the second analysis, the same variables were used to predict SM. In the third analysis, peak values per hyper-bar were used to predict average emotion measure per hyper-bar. All analyses were done directly and with a time-delay of one, two and three quarter notes of the performance data with respect to the listeners' data. The best $\mathrm{R}^{2}$ obtained will be reported. These were obtained with a delay of either zero or one for SM, and either two or three for the EM.

$\mathrm{SM}$ was rather well-predicted by the model, given the $\mathrm{Rs}^{2}$ of $0.34,0.33$, 0.30 for $\mathrm{p} 1, \mathrm{p} 2$ and $\mathrm{p} 3$, respectively. From this model, IOI was the only significant variable. In other words, duration was a fairly good predictor of the variation in number of participants indicating a section-boundary. More participants indicated a phrase-boundary for longer durations.

EM was well-predicted by the quarter note model, but even better by the second model that took the peak values per hyper-bar to predict the average EM per hyper-bar. The quarter note regression analysis had an $\mathrm{R}^{2}$ of 0.45 , $0.68,0.50$ for $\mathrm{p} 1, \mathrm{p} 2$, and $\mathrm{p} 3$, respectively, while the hyper-bar peak value regression had an $\mathrm{R}^{2}$ of $0.53,0.82$, and 0.56 . Velocity was always the most significant variable, and was the only significant variable for the hyper-bar peak value regression. For the quarter note regression movement velocity also reached significance for $\mathrm{p} 2$ and $\mathrm{p} 3$, and IOI for $\mathrm{p} 2$. All $\mathrm{Rs}^{2}$ are relatively high for $\mathrm{p} 2$, which suggests that the exaggerated expression of this performance increased communication.

As a comparison, the analyses were repeated with x-position and y-position as independent movement variables instead of the more general movement velocity variable. The results did not improve or change from this alteration, instead $\mathrm{x}$ and $\mathrm{y}$-position did not contribute significantly to any of the regressions.

These results confirm a differentiation between expressive means: tempo primarily communicates segmentation, while dynamics communicates emotional intensity. Velocity of the movement is correlated with dynamics and may therefore also reflect emotional intensity, but the sounding parameters are the main communicative factors.

The results are suggestive counter-evidence for hypothesis 4 . The failure of tempo to explain variations in emotional intensity contradicts the theory that phrase-final lengthenings cause a release in emotional intensity. There is, however, another way in which phrasing and the dynamics of tension and release do relate, which is at a higher and more global level. Phrase-final lengthenings occur at a high rate and a local scale. At this local scale the relation is weak. Major phrase boundaries that are indicated by a drop in tempo and dynamics are, however followed by a clear release in intensity. Moreover

\section{8}


the global variation of dynamics to which the variation in emotional intensity is so strongly related is the performer's way of communication of the overall form: the first part is an introduction and builds up to the B section, which he considers as the real beginning of the piece. This beginning is again a preparation for the first target of the piece: the release of tension in the middle of the B section. ${ }^{27}$ Hereafter, tension builds up towards the dramatic return of the theme, which leads via a repeat of the theme in contrasting dynamics to the second important target of the theme: the second possible release of tension at the general pause. After the general pause, the release is not given and all hope is lost. The piece ends most sadly. The pianist most skilfully expresses this interpretation in the patterning of dynamics. ${ }^{28}$ The resulting phrasing is over the entire piece, with subdivisions at measures 22 and 36. The return of the theme is the culminating point of the piece, whereafter tension can release. According to the pianist, this tension cannot, however, be fully resolved.

\subsection{Summary}

This study had two aims: (i) individuating which auditory and visual cues are most involved in conveying the performer's expressive intentions and (ii) testing the model developed by comparing their performances with spectators' rating of the same musical performances. The auditory and visual cues most involved in conveying the performer's expressive intentions were hypothesised to be key velocity, IOI, movement velocity, and the openness or contraction of the performer's posture. In addition, a relation between phrasing and emotional tension-release was expected.

The analyses of performance data suggested the opposite relation between emotional intensity and the performer's posture. The pianist leaned forward for softer passages and backward for intensive passages. In addition it suggested a differentiation in expressive means, with tempo on one side, and key velocity and movement velocity on the other side.

When relating the performer's data to the listeners' data, this differentiation in expressive means was confirmed. Tempo communicates phrase boundaries, while dynamics is highly predictive for the intensity of emotion felt. Emotional engagement correlated strongly with key velocity, which means that emotional engagement tended to increase with increase of dynamics and decrease at points of softer dynamics. This does not mean that soft passages were without emotional tension, but they were points of relative emotional relaxation. Hardly any evidence was found for movement cues influencing

27 See downward pointing arrows in Fig. 3.

28 See arrows in the key velocity panel of Fig. 3. 
listeners' ratings. The sound seemed to be the primary focus of the participants, and vision seemed subsidiary. The local phrase boundaries indicated by tempo did not lead to a release of emotional intensity. The modulation of dynamics over a larger time-span communicates the overall form of the piece and, at that level, intensity did increase and decrease within phrases.

\section{Applications of Multimodal Expressive Systems: the Case Study of Active Music Listening}

Music making and listening are a clear example of a human activity that is above all interactive and social. However, nowadays mediated music making and listening is usually still a passive, non-interactive, and non-contextsensitive experience. The current electronic technologies, with all their potential for interactivity and communication, have not yet been able to support and promote this essential aspect of music making and listening. This can be considered a degradation of the traditional listening experience, in which the public can interact in many ways with performers to modify the expressive features of a piece.

The need to recover such an active attitude with respect to music is emerging strongly, and novel paradigms of active experience will be developed. By active experience and active listening we mean that listeners are enabled to interactively operate on music content, by modifying and molding it in real-time while listening. Active listening is the basic concept for a novel generation of interactive music systems, which are particularly addressed to a public of beginners, naïve and inexperienced users, rather than to professional musicians and composers.

Active listening is also a major focus for the new EU-ICT Project SAME (Sound and Music for Everyone, Everyday, Everywhere, Every Way). ${ }^{29}$ SAME aims at: (i) defining and developing an innovative networked end-to-end research platform for novel mobile music applications, allowing new forms of participative, experience-centric, context-aware, social, shared, active listening of music; (ii) investigating and implementing novel paradigms for natural, expressive/emotional multimodal interfaces, empowering the user to influence, interact, mold, and shape the music content, by intervening actively and physically into the experience; and (iii) developing new mobile contextaware music applications, starting from the active listening paradigm, which will bring back the social and interactive aspects of music to our information technology age. 
In the direction of defining novel active listening paradigms, we recently developed a system, the Orchestra Explorer, ${ }^{30}$ allowing users to physically navigate inside a virtual orchestra, to actively explore the music piece the orchestra is playing, and to modify and mold in real-time the musical performance through expressive full-body movement and gesture. By walking and moving on the surface, the user discovers each single instrument and can operate through her expressive gestures on the musical piece which the instrument is playing. The interaction paradigm developed in the Orchestra Explorer is strongly based on the concept of navigation in a physical space where the orchestra instruments are placed. The Orchestra Explorer is intended for use by a single user.

Our novel multimodal system for social active listening, Mappe per Affetti Erranti, starts from the Orchestra Explorer and the lessons learned in over one year of permanent installation of the Orchestra Explorer at our site at Casa Paganini, and several installations of the Orchestra Explorer at science exhibitions and public events.

Mappe per Affetti Erranti extends and enhances the Orchestra Explorer in two major directions. On the one hand it reworks and extends the concept of navigation by introducing multiple levels: from the navigation in a

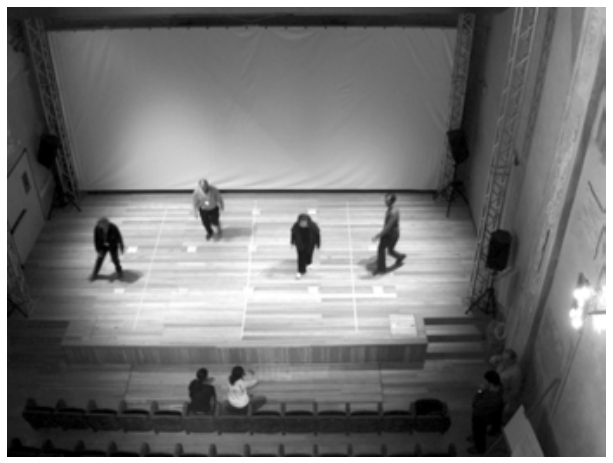

Fig. 5. A group of users interacting with the installation "Mappe per Affetti Erranti" at the auditorium of Casa Paganini. physical space populated by virtual objects or subjects (as it is in the Orchestra Explorer) up to the navigation in virtual affective, emotional spaces populated by different expressive performances of the same music piece. Users can navigate in such affective spaces by their expressive movement and gesture. On the other hand, Mappe per Affetti Erranti is explicitly designed for use by multiple users, and encourages collaborative behaviour: only social collaboration allows a correct reconstruction of the music piece. In other words, while users explore the physical space, the (expressive) way in which they move and the degree of collaboration between them allow them to explore at the same time an affective, emotional space.

30 Camurri/Canepa/Volpe 2007 
The basic concept of Mappe per Affetti Erranti is the collaborative active listening of a music piece through the navigation of maps at multiple levels, from the physical level to the emotional level.

At the physical level the space is divided in several areas. The voice of a polyphonic music piece is associated to each area. The presence of a user (even a single user) triggers the reproduction of the music piece. By exploring the space, the user walks through several areas and listens to the single voices separately. If the users stays in a single area, she listens to the voice associated to that area only. If the user does not move for a given time interval, the music fades out and turns off.

The user can mold the voice she is listening to in several ways. At a low level, she can intervene on parameters such as loudness, density, amount of reverberation. For example, by opening her arms, the user can increase the density of the voice (she listens to two or more voices in unison). If she moves toward the back of the stage the amount of reverberation increases, whereas toward the front of the stage the voice becomes drier.

At a higher level the user can intervene on the expressive features of the music performance. This is done through the navigation of an emotional, affective space. The system analyses the expressive intention which the user conveys with her expressive movement and gesture, and translates it in a position (or a trajectory) in an affective, emotional space. Like the physical space, such affective, emotional space is divided in several areas, each one corresponding to a different performance of the same voice with a different expressive intention. Several examples of such affective, emotional spaces are available in the literature, for example the spaces used in dimensional theories of emotion ${ }^{31}$ or those especially developed for the analysis and synthesis of expressive music performance. ${ }^{32}$

Users can thus explore the musical piece in a twofold perspective: navigating the physical space they explore the polyphonic musical structure; navigating the affective, emotional space they explore music performance. A single user, however, can only listen to and intervene on a single voice at a time: she cannot listen to the whole polyphonic piece with all the voices.

Only a group of users can fully experience Mappe per Affetti Erranti. In particular, the musical piece can be listened to in its whole polyphony only if a number of users at least equal to the number of voices is interacting with the installation. Moreover, since each user controls the performance of the voice associated to the area she occupies, the whole piece is performed with the same expressive intention only if all the users are moving with the same expressive intention. Thus, the more users move with different, conflicting

31 See for example Russel 1980; Tellegen et al. 1999.

32 See for example Juslin 2000; Canazza et al. 2000; Vines et al. 2005.

\section{2}


expressive intentions, the more the musical output is incoherent and chaotic. But the more users move with similar expressive intentions and in a collaborative way, the more the musical output is coherent and the musical piece is listened to in one of its different expressive performances.

Mappe per Affetti Erranti can therefore be experienced at several levels: by a single user who has a limited but still powerful set of possibilities of interaction, by a group of users who can fully experience the installation, and by multiple groups of users. In fact, each physical area can be occupied by a group of users. In this case each single group is analysed and each participant in a group contributes towards intervening on the voice associated to the area the group is occupying. Therefore, at this level a collaborative behaviour is encouraged among the participants in each single group and among the groups participating in the installation.

The possibility of observing a group or multiple groups of users during their interaction with Mappe per Affetti Erranti makes this installation an ideal test-bed for investigating and experimenting group dynamics and social network scenarios.

\section{Discussion}

The modelling of expressive gesture is being accorded growing importance from both research and industry communities, even if we can consider it as being in its infancy. The main outputs of our research are the definition of a unified multimodal conceptual framework for expressive gesture processing, the experimental results obtained from the two described experiments, and a collection of software modules for cue extraction and processing. The conceptual framework proved to be useful and effective in two different scenarios, well represented by the two experiments described in the paper.

In the first experiment, we focused on the communication of basic emotions from a dancer to the audience, while in the second experiment we focused on the mechanisms that possibly cause emotional engagement in the audience.

The dance experiment can be considered as a first step and a starting point toward understanding the mechanisms of expressive gesture communication in dance. A collection of cues that have some influence in such a communication process was individuated, measured, and studied. A first attempt of automatic classification of motion phases was carried out and some results were obtained (e.g., an average rate of correct classification which was not particularly high, but well above chance level). Some directions for future research have also emerged. For example, other classification techniques could be employed and their performances compared with what 
we obtained with decision trees. Some aspects in dance performance that were only marginally considered should be taken into account. In particular, aspects related to rhythm should be further investigated. Expressive cues such as impulsiveness and fluency should be further worked out. Moreover, perceptual experiments would be needed to empirically validate the expressive cues extracted.

The music experiment can be considered as a first step towards the understanding of the relation between movement and sound parameters of a performance, their expressive forms and functions, and their communicative function for spectators. A next step should involve a larger variety of performances and a larger collection of calculated cues, and cues should be fitted to the responses of individual spectators in order to get a deeper as well as broader understanding.

Expressive multimodal systems open a novel range of applications. At the end of this chapter we focused on an application in the field of active music listening. 


\section{References}

Bobick, Aaron F./Davis, James W. (2001): "The Recognition of Human Movement Using Temporal Templates«. IEEE Transactions on Pattern Analysis and Machine Intelligence 23(3), 257-267.

Boone R. Thomas / Cunningham Joseph G. (1998): „Children's Decoding of Emotion in Expressive Body Movement: The Development of Cue Attunement«. Developmental Psychology 34, 1007-1016.

Bradsky, Gary R. / Davis, James W. (2002): »Motion Segmentation and Pose Recognition with Motion History Gradients«. Machine Vision and Applications 13, 174-184.

Camurri, Antonio et al. (2000): »EyesWeb - Toward Gesture and Affect Recognition in Interactive Dance and Music Systems«. Computer Music Journal 24(1), 57-69.

Camurri, Antonio/Lagerlöf, Ingrid/Volpe, Gualtiero (2003): »Emotions and Cue Extraction from Dance Movements«. International Journal of Human Computer Studies, 59(1-2), 213-225.

Camurri Antonio/Mazzarino, Barbara/Volpe, Gualtiero (2004): »Analysis of Expressive Gesture: The Eyesweb Expressive Gesture Processing Library«. In: Antonio. Camurri/ Gualtiero Volpe (Eds.), Gesture-based Communication in Human-Computer Interaction, GW’08, LNAI 2915, Berlin: Springer, 2004, 460-467.

Camurri, Antonio et al. (2005): »Toward Communicating Expressiveness and Affect in Multimodal Interactive Systems for Performing Art and Cultural Applications«. IEEE Multimedia 12(1), 43-53.

Camurri, Antonio/Canepa, Corrado/Volpe, Gualtiero (2007): »Active Listening to a Virtual Orchestra through an Expressive Gestural Interface: The Orchestra Explorer«. In: Proceedings of the 2007 Conference on New Interfaces for Musical Expression (NIME07), 56-61.

Canazza, Sergio et al. (2000): »Audio Morphing Different Expressive Intentions for Multimedia Systems«. IEEE Multimedia, 7(3), 79-83.

Chi, Diane/Costa, Monica/Zhao, Liwei/Badler, Norman (2000): »The EMOTE Model for Effort and Shape«. In: John S. Brown/Kurt Akeley (Eds.) Proceedings of the 27th Annual Conference on Computer Graphics and Interactive Techniques, New York: ACM Press/Addison-Wesley Publishing Co., 173-182.

Cowie, Roddy et al. (2001): »Emotion Recognition in Human-Computer Interaction«. IEEE Signal Processing Magazine 1, 33-80.

Friberg, Anders et al. (2000), »Generating Musical Performances with Director Musices«. Computer Music Journal 24(3), 23-29.

Hashimoto, Shuji (1997): »KANSEI as the Third Target of Information Processing and Related Topics in Japan«. In: Antonio Camurri (Ed.), Proceedings of the International Workshop on KANSEI: The Technology of Emotion, Genova: AIMI (Italian Computer Music Association) and DIST-University of Genova, 101-104. 
Juslin, Patrik N. (2000): »Cue Utilization in Communication of Emotion in Music Performance: Relating Performance to Perception«. Journal of Experimental Psychology: Human Perception and Performance 26/6, 1797-1813.

Kilian, Johannes (2001): »Simple Image Analysis By Moments«, OpenCV library documentation. Online available in the repository of the OpenCV Yahoo group (last access: July 2008).

Krumhansl, Carol L. (1996): »A Perceptual Analysis of Mozart's Piano Sonata K. 282: Segmentation, Tension and Musical Ideas«. Music Perception 13(3), 401-432.

Krumhansl, Carol L./Schenck, Diane L. (1997): »Can Dance Reflect the Structural and Expressive Qualities of Music? A Perceptual Experiment on Balanchine's Choreography of Mozart's Divertimento No. 15«. Musicae Scientiae 1, 63-85.

Kurtenbach, Gordon/Hulteen, Eric A. (1990): „Gesture in Human-Computer Interaction«. In: Benda Laurel (Ed.), The Art of Human-Computer Interface Design, Reading: Addison-Wesley, 309-317.

Laban, Rudolf (1963): Modern Educational Dance, London: Macdonald \& Evans.

Laban, Rudolf/Lawrence, F. C. (1947): Effort, London: Macdonald \& Evans.

Lagerlöf, Ingrid/Djerf, Marie (2001): On Cue Utilization for Emotion Expression in Dance Movements, Manuscript in preparation, Department of Psychology, University of Uppsala.

Liu, Yanxi/Collins, Robert T./Tsin, Yanghai (2002): »Gait Sequence Analysis using Frieze Patterns«. In: Anders Heyden/Gunnar Sparr/Mads Nielsen/Peter Johansen (Eds.), Computer Vision - ECCV 2002, 7th European Conference on Computer Vision, New York: Springer, 657-671.

Lucas, Bruce D./Kanade, Takeo (1981): »An iterative image registration technique with an application to stereo vision«. In: Patrick J. Hayes (Proceedings of the 7th International Joint Conference on Artificial Intelligence (IJCAI '81), Los Altos: William Kaufmann, 674-679.

McNeill, David (1992): Hand and Mind: What Gestures Reveal About Thought, Chicago/ London: University of Chicago Press.

Palmer, Caroline (1997): »Music Performance«. Annual Review of Psychology 48, $115-138$.

Russell, James A. (1980): »A Circumplex Model of Affect«. Journal of Personality and Social Psychology 39, 1161-1178.

Pollick, Frank E. et al. (2001): »Perceiving Affect from Arm Movement«. Cognition 82(2), B51-B61.

Tellegen, Auke (1999): »Watson David, and Clark Lee Anna. On the Dimensional and Hierarchical Structure of Affect «. Psychological Science 10(4), 297-303.

Timmers, Renee (2002): Freedom and Constraints in Timing and Ornamentation: Investigations of Music Performance, Maastricht: Shaker Publishing.

Timmers, Renee et al. (2006): »Listeners' Emotional Engagement with Performances of a Skriabin Etude: An Explorative Case Study«. Psychology of Music 34(4), 481-510.

\section{6}


Vines Bradley W. et al. (2005): „Dimensions of Emotion in Expressive Musical Performance«. Ann. N.Y. Acad. Sci., 1060, 462-466.

Volpe, Gualtiero (2003): Computational Models of Expressive Gesture in Multimedia Systems. PhD Thesis, Faculty of Engineering, University of Genova, April 2003.

Wanderley, Marcelo M./Battier, Marc (Eds.) (2000): Trends in Gestural Control of Music, Paris: IRCAM.

Wilson, Andrew. D./Bobick, Aaron. F./Cassell, Justine (1996): Proceedings of the Second International Conference on Automatic Face and Gesture Recognition, 1996, 14-16 Oct, 66-71.

Zhao, Liwei (2001): Synthesis and Acquisition of Laban Movement Analysis Qualitative Parameters for Communicative Gestures, PhD Thesis, University of Pennsylvania.

\section{Acknowledgements}

We thank Ingrid Lagerlöf for the joint work carried out in the experiment on expressive gesture conveying emotions in dance performances, Renee Timmers and Matja Marolt for their contribution to the experiment on music performance, our colleagues at the InfoMus Lab, and the pianist Massimiliano Damerini for his artistic contributions in providing the material for the piano studies.

The novel applications on active music listening are the main objective of the EU-ICT Project SAME33, focusing on new forms of participative, context-aware, socially active listening to music. 


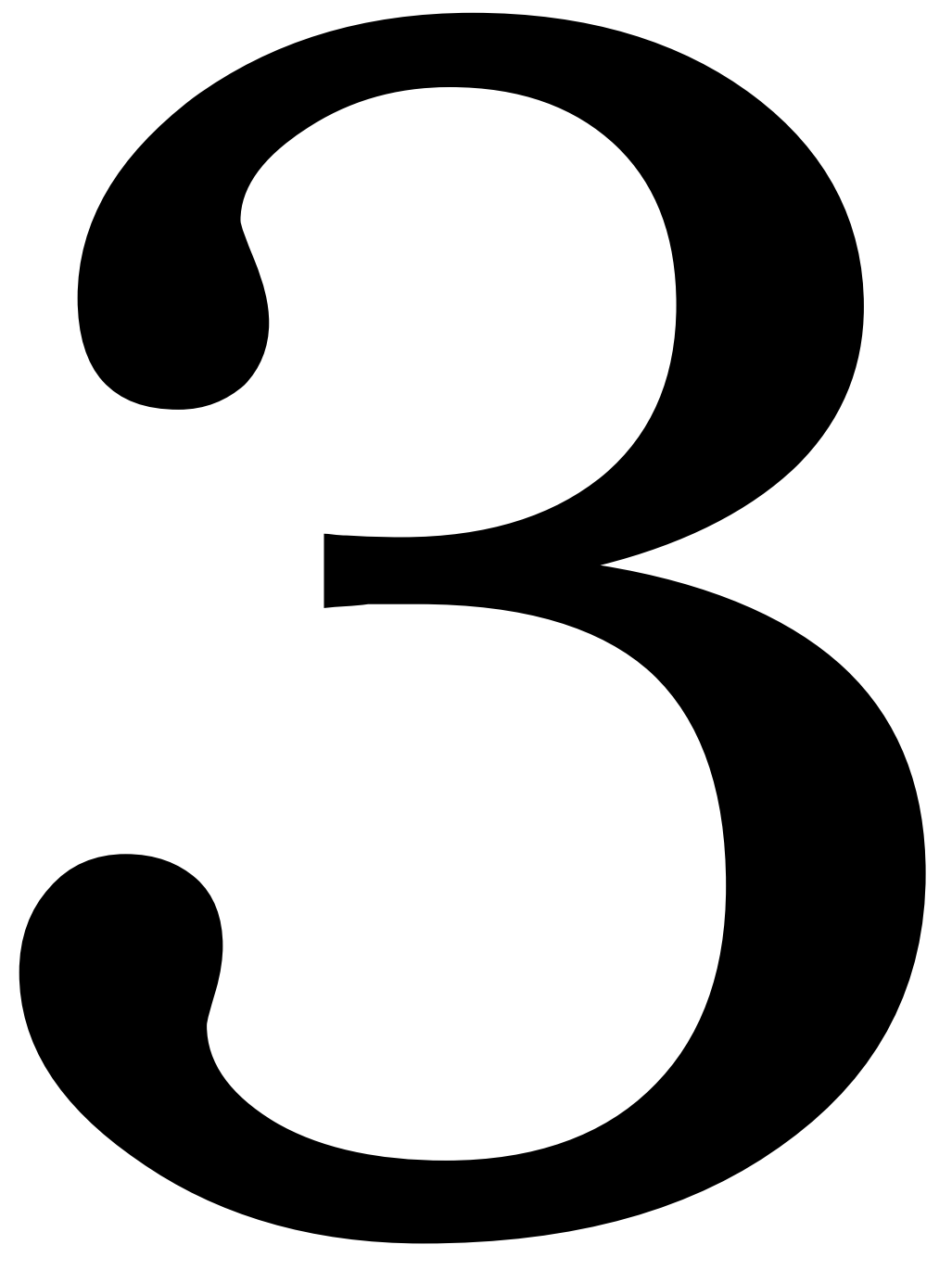




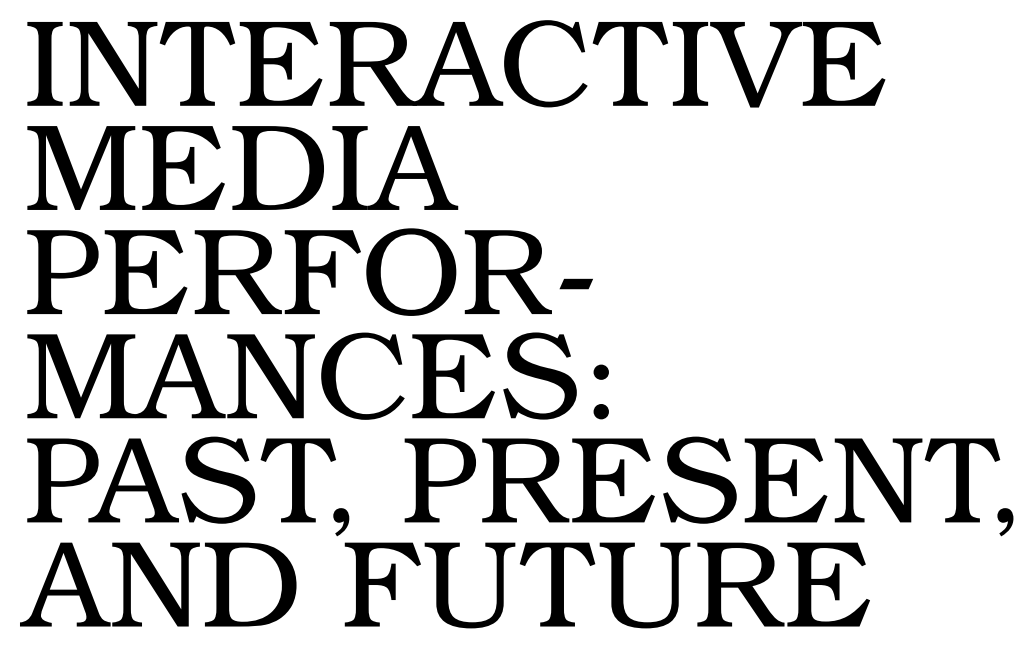




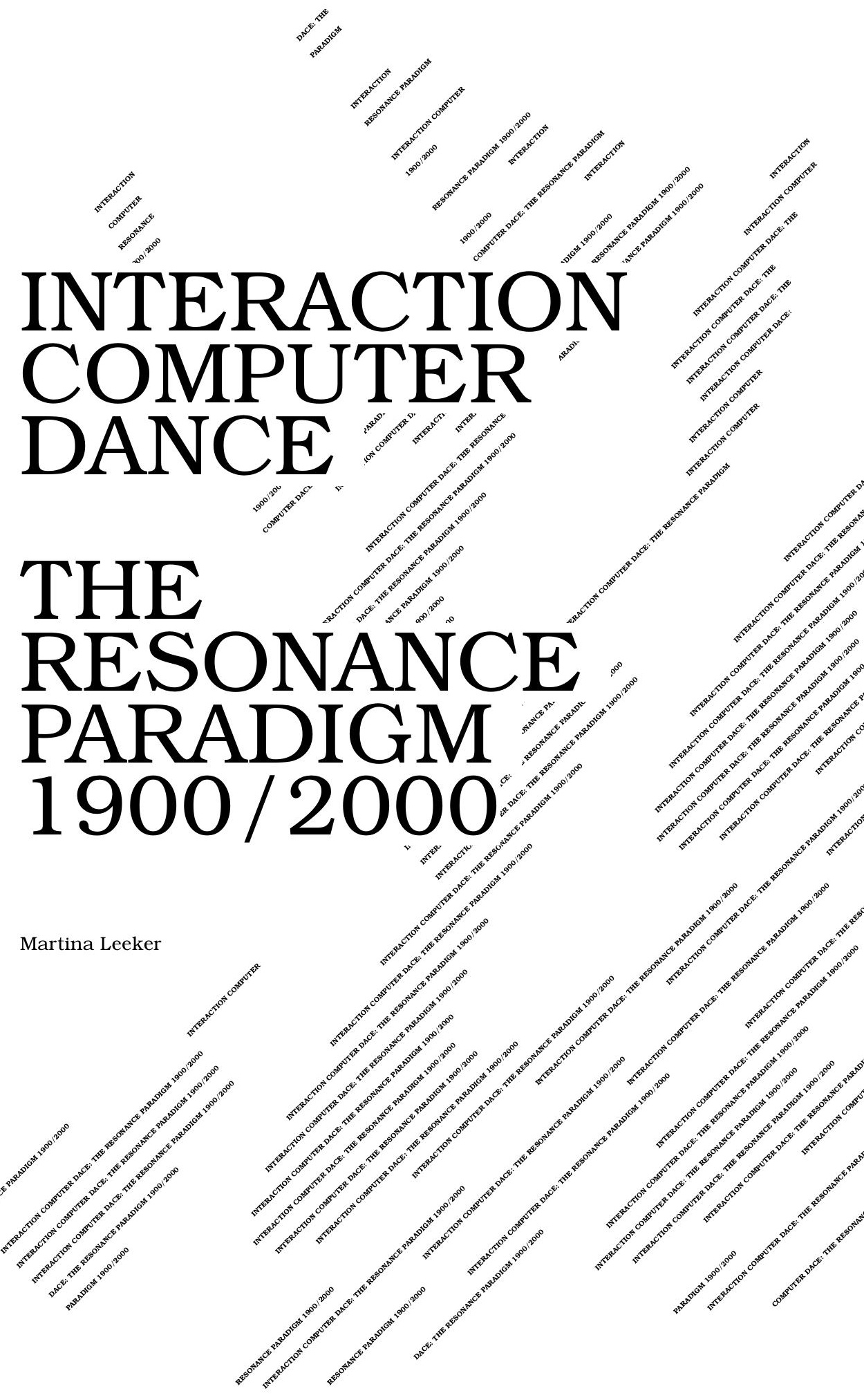


All movement causes oscillation, yet we lack the ears to hear it. Marin Mersenne

The subjects of this paper are theater/performance formats of contemporary dance as exemplary instances of the interaction between people and media. Thus, practices of dancing with computers and the generation of music and sound in so-called real-time are interrogated. ${ }^{1}$ This entails the discussion of situations where the impression occurs that dancers create "their" own music by their movements.

I intend to consider contemporary practices of dancing with computers in their relation to a highly problematic historical discourse. Central to this discourse is the paradigm of a resonance between human beings and media constituted around 1900, which emerges through technical media and within the history of knowledge these technical media are based on. A frame of reference for the Resonance Paradigm ${ }^{2}$ is the encounter with electricity in the course of electromagnetic research. In this shape, it is the basis of signal theory in physics as well as in the experimental human sciences, the emerging physiology. While many of the phenomena observed in electromagnetic research could not be scientifically explained at the time, they were implemented in technology used by media such as the telegraph or cinematography. ${ }^{3}$ This gap between knowledge and technology frames the discourse of an "Aether Physics" influenced by spiritism. Aether Physics provides the foundation for the Resonance Paradigm, which - in turn - produces newly invented, overstimulated "trigger-bodies" (Schaltkörper) dancing on the stages of theaters. Crucial for a critical reading of the Resonance Paradigm is the masking and camouflage it provides for an epistemic shift induced by electromagnetic research: from a conduction model of electricity to induction and thus to a notion of signal processing beyond language and meaning. This shift is masked by various suppositions of similarities between human beings, devices, and media on the one hand and the superimposition of the non-human with spiritism on the other hand, thus opening a channel of communication between a para-physical world and humanity.

1 Good examples of contemporary practice are the works of blackhole-factory (2001).

2 Resonance occurs, once at least two entities start to oscillate and once they are oscillating in the same frequency amplify each other's vibration, which presupposes a similarity of the participating entities.

3 About film as scanning and signal processing, see Siegert (2006a) and Herrmann (1996).
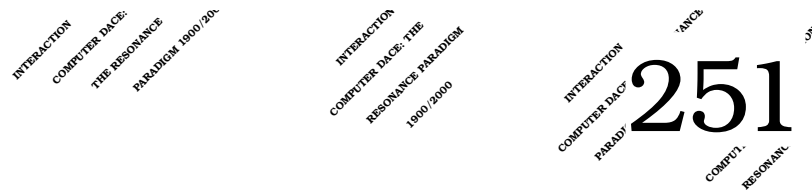


\section{Resonances in Technical Media around 1900 and the Interaction with Computers}

Around 1900, both discourse networks ${ }^{4}$ and dance come into the reach of technical media such as phonography, cinematography and telegraphy, which allow the notation of the physically real. All of a sudden, the acoustic and the visual are frequencies. Entities that had been simply non-recordable and in-visible, now take shape according to the properties of new technical media. Simultaneously with these "new" media and new devices, dancers appear whose claim for the practice of dancing rests in their lack of formal training, since this very lack best suits a new kind of dance. Forms of dance emerge which are not touched by language but are constituted by sheer physiology, by a transmission of stimuli from body to body. In this context, dance and music turn into continuous imprints on the physically real and, furthermore, to options for regulating and optimising hitherto uncontrollable, preverbal organicity.

\section{Ma(g)deleine Guipet: \\ Automaton of Reflexes and a Physiological Aesthetics}

Madeleine Guipet may well be seen as the defining instance of a new relation between dance and music, created and practiced in the realm of electron physics and spiritism. She leads the way in the transposition of the organic into technical media ${ }^{5}$, into trigger-bodies ${ }^{6}$. Guipet belongs to a group of hysterics, who had become something of the great white hope for an intensification of physical presence and the heightening of physical abilities. The female hysterics are seen as over-stimulated and over-sensitive subjects whose pathological disposition makes them resonate with the whole of their environment. Somewhat drastically, Albert von Schrenck-Notzing, the physician in charge of Mlle. Guipet's therapy, calls her a "somnambulist automaton of reflexes." From the 1870s on, the French psychiatrist Jean-Marie Charcot uses hypnosis, the newly established experimental science, to draw the female medium into a state of trance, thus switching off cognitive control.

4 The term "discourse networks" has been used to translate Friedrich Kittlers notion of "Aufschreibesysteme" (Kittler 1985) apparently for reasons of resonance with Foucaultian terminology (Wellberry 1992, p. XI). A more literal translation would read "systems of writing down" or "notation systems" (note of the translator).

5 The notion of "technical media" is not meant metaphorically here, but in a very specifically technical sense. Bodies turn into technical media by being defined and addressed by contemporary automatons of experimental physics and physiology.

6 Georges Didi-Huberman (2004, p. 192) introduces this term in his discussions of "Repetitions, Rehearsals, Staging" (pp. 175-257) as elements of the photographic practices in Charcot's Salpêtière.

7 von Schrenck-Notzing 1904, p. 117

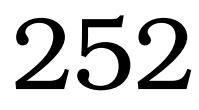


In this way, hypnosis turns into a controlling function of the human power plant of hysteria, since it turns the hitherto uncontrollably twitching ladies, who had been interesting for their intensified stimulatedness and sensitivity, into "trigger-bodies" where an archive of gestures and facial expressions could be retrieved and made visible in a precise manner. ${ }^{8}$ If the human body had up to his point primarily been the site of language, of the subject and the soul, it now turns into a terrain of technically reproducible command circuits; a constitution that humans share with animals and telegraphs.

Madeleine Guipet is put into a trance on stage; ${ }^{9}$ she is exposed to piano music, which - in the eyes of her physicians and her audience around 1904 - forces her to dance like a puppet on strings. ${ }^{10}$ The theory explaining this phenomenon claims that the sound of the instrument consists of frequencies, which induce oscillation in the body of the medium, thereby activating programs of stimulation stored in the body as neurological circuits. Madeleine is celebrated as the perfect example of the sensitised body and by extension, of the human potential for retrieving and unlocking ever-increasing powers of perception and knowledge.

In a Nietzschean vein, Madeleine Guipet could be framed as evidence for a physiological aesthetics, an aesthetics constituted beyond language by being a pure transference of stimuli from body to body. ${ }^{11}$ Symbolic bodies thus turn into technical media, into trigger-bodies, and this is the final frontier of dance as an art form, since it is not art that is at stake here any longer, but the intensification of stimuli and biopolitics. ${ }^{12}$

\section{Epistemic Shifts: Electromagnetism and Spiritism}

Studying the (false) belief in a resonance between people and media, which also echoes McLuhan's notion of "media as extension of man" in mid $20^{\text {th }}$ century media studies, ${ }^{13}$ reveals the epistemic situation of the late $19^{\text {th }}$ century. The Resonance Paradigm emerges at a time of revolutionary changes in both physics and media technologies, which in turn foster insecurities and shifts in philosophical and epistemological means and concepts. In my view,

\footnotetext{
8 Herrmann 2005, p. 191

9 Bahr 1999

10 Herrmann 2005, pp. 202-211

11 Herrmann 2005, pp. 195-200

12 Hans-Christian von Herrmann notes that this increase in potential has always been a point of entry for biopolitics. If phenomena which had not been measurable turn into subjects for notation, they may also be reproduced and turned into training devices. Evidence for this dynamics may be found in the research and practical methods developed with the cinematograph and chronophotography by Frank Bunker Gilbreth in the context of ergonomics.
}

13 Leeker 2008 
the Resonance Paradigm - fueled by spiritist insanity - bridges two epistemologies. It serves the purpose of blunting the impact of a cultural revolution by reworking notions of the anthropological ${ }^{14}$ and by the "expulsion of spirit" (Austreibung des Geistes) ${ }^{15}$.

The substantial turn at issue here is based on electromagnetic research. ${ }^{16}$ Its initial guiding question was an explanation of electromagnetism in accordance with calculable and predictable laws of nature - such as the law of conservation of mass/matter - as they had been known up to this point. The discovery of electromagnetic sparks by Heinrich Hertz (1886-1888) raises issues that cannot be resolved for the time being, since the spark leaps over a gap without being conducted by any materially specified substance. This phenomenon raises not only the all-decisive philosophical issue if and how the universe and the world could still be observed and comprehended. It also raises the question, whether a world in which something belonging to nature can leap may still be explained by one, spiritual, and all-encompassing principle. If electrons may be waves as well as particles, then - thus the inconvenient truth - the traditional laws of classical Newtonian physics could perhaps not explain them and neither could they be calculated by a mathematics ${ }^{17}$ believing in its reference to the world. ${ }^{18}$

This loss of direct access to the world occurs on several levels. Crucial for an understanding of dance and music around 1900 for instance, is Hermann von Helmholtz' research about the "Sensations of Tone" in the 1870s. It demonstrates that it is not the world that is heard but the brain itself, so that the

\section{Siegert $2006 \mathrm{~b}$}

15 See Friedrich Kittlers Austreibung des Geistes aus der Geisteswissenschaft (1980) where he laid the foundations for his media theory, namely the provocative argument of a mediatechnological apriori of anthropology and culture. The situation around 1900 is related to this notion, not in terms of media technology, however, but in the practices of camouflaging machines and as a discourse of its own. Major dramaturgical efforts are necessary, after all, to deliver human beings to the machine.

\section{Hagen 2005}

17 The role of mathematics within the turn to a theoretical physics of approximation and uncertainty relations initiated by electromagnetism can only be sketched here. At first, mathematics obstructs the inevitable paradigm shift. Although its crisis of foundation turns it towards a self-referential axiomatic model (Hilbert), mathematics also comes up with the new metaphysical concept of the inclusive calculability of the world. Suffice to mention, that Cantor presented in 1880 the Mannigfaltigkeitslehre (known today as set theory), which tries to calculate (aether's) infinity and make it computable. Hilbert's Axiomatic Mathematics, as well as the formal logic that rose from it and became the basis for computers, continue this tradition of abstraction and formalisation towards the ends of calculating the infinite and purely spiritual (and thus of the aether). For an introduction to the interplay of physics and mathematics in the context of spiritist ways of thinking, see Hagen (1995/96).

18 According to Wolfgang Hagen, the discovery and exploration of electromagnetism was a turning point that turned thinking and knowledge away from the philosophy of nature and towards a world of approximations and uncertainty relations, where world and formal systems co-exist side by side.

\section{4}


human body, its nervous system pervaded by electrical circuits, constitutes perception. ${ }^{19}$ If we had different ears, brains and nerves, we would be hearing differently. In the wake of Helmholtz, the issue of perception turns from the transport and passage of impulses to their transmission and to induction as an ensemble of triggering events and circuitry within the confines of previously stored programs. It is all about the control and the operation of information.

The phenomenon emerging in the realm of physiology, namely the separation of energy from information, is implemented in the media technologies of telecommunication. The terrain where insecurities raised by the epistemology of electromagnetism crystallise most tangibly is telegraphy, which emphatically articulates the bond between telecommunications technology and Aether Physics - the kind of physics that still believes in the existence of a spiritual and, at the same time, materially tangible soul of nature, despite emerging doubts.

Furthermore, telegraphy demonstrates that in information technology neither ghosts nor energies, neither rays nor currents are being switched, but only information. And similar to electrons, information has no business with the anthropologically meaningful. If Helmholtz had already reduced the sense of tone to electrical impulses whose fate was bound to the facilities of the brain, the separation of energy and information finally creates an empire fundamentally at odds with human facilities of comprehension, since meaning is nothing and signals are all. Any relation of signifier and signified is severed and communication may no longer be comprehended in terms of comprehension but merely as transport of signals.

This new disposition places human beings in an uncertain position between modalities of conduction and switching, between spirit and information - a site emerging from the differentiation of energy and signal, of matter and information in electrical processes. The potentially threatening effect of the electrically electronic media - that is the technical media - is an erosion of the anthropomorphous. They create a realm framed by analogue signals, which could still be human and thus comprehensible (not the least because they make him or her dance), and information, which human beings cannot comprehend any more. Human existence thus turns into a co-existence with self-organising machines and with White Noise, where meaning is either some kind of accident or a result of programming.

It is this situation, where Aether Physics comes to the rescue since it establishes connections between media, physics, experimental sciences and spiritism, which bring incomprehensible media and their momentum of with-

19 For the importance of resonance or "Mittönen" in Helmholtz' psychophysiology of hearing on the basis of soundwaves and Fourier analysis as discrete formations with no orientation in meaning, see Volmar (2003). 
drawal from human comprehension into a horizon of explanation. While this horizon is in itself not rationally comprehensible, (since it operates with phenomena of the extra sensual), it is potent and seemingly salutary for the strain of epistemological shifts. Within the awkward philosophical, theological, and media-technological situation, Aether Physics may be seen as an attempt to provide access to phenomena of electromagnetism no longer or not yet explainable by laws and methods of classical physics. Yet at the same time, Aether Physics claims to have evidence of a "fourth dimension" consisting of rays and fluids endowed with spiritual power, the aether indeed. Thus, William Crookes ${ }^{20}$ proclaims:

\begin{abstract}
It seems to me that in these rays we may have a possible mode of transmitting intelligence, which, with a few reasonable postulates, may supply a key to much that is obscure in psychical research. Let it be assumed that these rays, or rays of even higher frequency, can pass into the brain and act on some nervous centre there. Let it be conceived that the brain contains a centre which uses these rays as the vocal cords use sound vibrations ... and sends them out, with the velocity of light, to impinge on the receiving ganglion of another brain. In this way some, at least, of the phenomena of telepathy, and the transmission of intelligence from one sensitive to another through long distances, seem to come into the domain of law, and can be grasped. ${ }^{21}$
\end{abstract}

In favour of spirits and ghosts, which may neither be seen nor heard, the leaping sparks - as much as the electrons whose dynamics are as such invisible, yet leave traces of their operations on other, conducting media - are thus denied the status of being real by themselves. This resolves issues arising from the new technical media's seeming abilities to record the physically real itself - as opposed to the semiotic-hermeneutical systems of notation employed by writing and the notation of dance. Thus, the recordings of frequencies of voices, sound and movement in resonance with the human body are displaced into the Aether and explained as phenomena of spiritual and spectral transmissions - all implications of insanity and occult spiritualism included.

\title{
Aether Physics in Performance: Occultism and Bodies as Technical Media
}

The turn in media history and the history of knowledge from the physics of electricity and its epistemology to the era of communications technology

20 William Crookes was a physicist and chemist. Since he conducted experiments with vacuum tubes, he is widely credited for the discovery of the cathode ray tube. He also experimented with various spiritistic media and was a member of the Theosophic Society as well as president of the Society for Psychical Research. See Hagen (2005) and Siegert (2006c).

21 Crookes 1897, p. 338

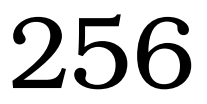


and Quantum Physics denies feasibility to traditional concepts maintaining a unified comprehension of and access to the world. Within the confines of this situation, the dancers of hysteria may well be read as bridging a gap between two epistemologies - albeit fuelled by spiritism and insanity. Their cultural productivity derives from their ability to prevent a complete, conceptual collapse of the human being by abolishing the anthropological altogether. The hysteric, musically-induced dancers may well be seen as hinges between energy and information. They are an attempt to conserve some of the Aether, before Axiomatic Mathematics, computers and Quantum Physics turn aside the electrified universe and the anthropological, or rather try to fuse both into the information machine.

Bodies as technical media achieve this delay, yet also break the ground for the integration of human beings into informatics: by the mediation of resonance and embeddedness in a model of oscillation contextualised in the occult on one hand, and by opening a passage towards the discrete, the transformation of the anthropological into information, on the other hand. Bodies as technical media thus provide a training ground for the trespassing of the threshold and simultaneously liquidate discreteness. The technological epitome of this risky position between energy and information is the relay, the switch, separating energy and information. ${ }^{22}$ Thus, bodies turn into relays or trigger-bodies. Yet inside of them, information also turns into oscillation, is triggered into resonances with the person and thus its ghostly apparition may still be humanly comprehensible and controllable. That this embodiment of media means to inscribe insanity into media, people and human-media, is the price to pay.

\section{Interaction and forced Immediacy: Digital Operations since 1960}

As we know from cybernetics, ${ }^{23}$ this scenario of camouflaging the history of knowledge, of epistemic shifts and media in the resonance paradigm, continues well after the actual switch to discrete processes in the mid-1940s. Human beings turn into information processing machines and cybernetics rises to the status of an all-encompassing discourse of explanation for humans, animals, learning, society, education, economy and culture. ${ }^{24}$ All these entities are similar in respect to the engineered control of self-organ-

22 Paradigmatic of this function are the relays at telegraph stations. By installing a relay on the side of the receiver, one does not have to send energy any more, but only an impulse, which hits the relay, moves it to switch a battery and thus close an electrical circuit for the transmission of signals.

23 Pias 2003

24 Pias 2004 
ised processes following the principles of a formal logic of classification and address-management by means of feedback. In order to explore the architecture of computers but also to construe interplay between bodies, perception and machines, many performances since the 1960s have turned to the Resonance Paradigm. Strategies and approaches that attempt to create resonance by means of camouflage are contemporary additional elements, while historic arguments referring to para-psychological events have ceased. ${ }^{25}$ Instead, the technological structure of computers is displaced ${ }^{26}$ by connecting electronic processes via imitation to the imagery and epistemology of hysteric media and dancers. This camouflage of the symbolic, universal machine with the aid of electronics has materialised since the 1940s in developments like interfaces and analogue/digital conversions. ${ }^{27}$ In addition, there has been a notable stress on performativity since the 1980s and more emphatically in the 1990s. Non-permanence, traces, intermedial transmission and transformation have gained importance, and to some extent even turned into ontologies of the technical materiality of computers as well as the anthropological. May we surmise then, that performativity as mediality and mediality as performativity have taken the place of spiritist and occult discourse ${ }^{28}$

\section{Echoes: The Resonance Paradigm and Contemporary Interactive Performances}

As mentioned earlier, contemporary dance performances with computers evoke the impression of dancers creating their own music by means of their movement. While these practices seem clearly opposed to the spasmodic electro-hysteric dance circuitry of the $19^{\text {th }}$ century, they may also be seen as modifications of hysteric dance for the age of the computer. Thus, the dancer is no longer "wired" to the music like Mlle. Guipet, rather it may seem that she or he is in control of the computer. In as much as the computer seems to

25 If spiritist hermeneutics have been debunked for good, it, however, remains a subject of discussion. Areas of research like biofeedback, where the circuitry of the brain is to be made visible, are somewhat suspicious. This practice would have to be questioned for its genesis from theosophical concepts like those of Leadbeater, who assumed different auras of human beings, which materialised in energies and colours.

26 Since the inauguration of cybernetics, this camouflage has lived off its own impossibility, since the analogue data of continuous organic processes cannot be transferred to modes of discrete scanning without considerable friction. Faster computing and scanning are needed, as well as a deception of perception by means of dramaturgies controlling attention and an aesthetics of imitating the electronic. See Hagen (2002a).

27 For a history of the ambivalence of analogue/digital conversion, see Schröter (2004).

28 For a discussion of the introduction of performativity as status of media into media and particularly computer studies via concepts like trace, process, transformation and contingency, see Krämer (1998, 2001). For a critical discussion of performativity, see Winkler (2004).

\section{8}


transform human movement into the dance preceding its acoustic coding, the impression occurs that the difference between notation and performance as well as the difference between dance and music have finally been overcome, since performance is writing itself down. Dance thus would return to its "essential being" - an ephemeral occurrence in time, which seems to embody processuality and transformation as anthropological ontology. Yet behind this discursive superimposition lures the fact that human beings and computers are fused to the same feedback loop.

Performances using so-called image/ sound processing in realtime are a case in point. In the realm of media art and performances with media, this setting has been popular since the 1960s. It has meanwhile consolidated into a standard design: analogue sensors gather data of physical systems and, via the MIDI protocol for analogue/digital conversion, a computer calculates this data. In the course of processing, transformations of images and sounds are generated and

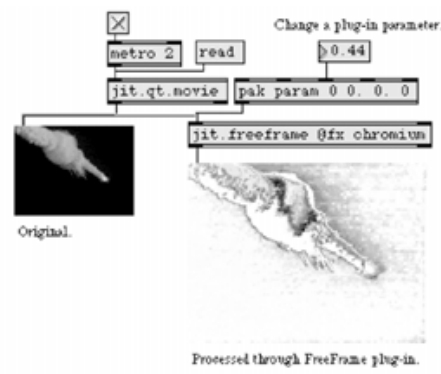

Fig. 1. Max/MSP/Jitter patch example $1 a$ passed on to analogue electronic devices, so that the computed changes are turned into output that is accessible to sensory experience. A program controls the so-called image and sound processing in realtime and this program also creates the illusion of interaction. ${ }^{29}$ Since the mid 1990s, the most popular software for this purpose has been Max/MSP/ Jitter (Fig. 1 and Fig. 2). ${ }^{30}$

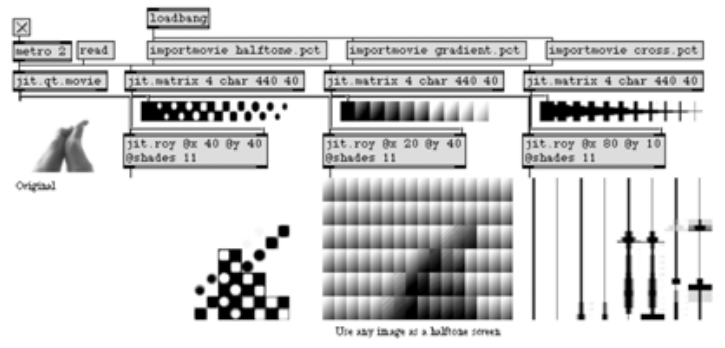

Fig. 2. Max/MSP/Jitter patch example 2

The Resonance Paradigm, based in spiritism and Aether Physics as it is, can hardly be articulated more blatantly. Assisted by McLuhan's notion of media as externalisations and extensions of human beings, these contem-

29 Leeker 2005

30 See <http: / / www.cycling74.com/products/maxmsp>. 
porary practices pass on the insanity of those dancers in hysteria who connected to machines around $1900 .^{31}$

\section{Technology Suppressed: \\ On the Difference between Recursion and Mapping}

A closer look at the technologies involved however shows that the coding of human motion according to statistical data is thoroughly suppressed in this setting. Suppressed and made invisible is, for instance, that it is the recording of movement with digital videocameras that generates this "music in motion". Video images are read into computers as an analysis of differences to be mapped on (pre-)programmed sets of data, which then are turned into outputs of acoustic phenomena. Just like the electrical-electronic experiments in motion research around 1900, this contemporary practice has very little to do with motion. Instead, we face a quite thorough transformation of motion by means of its discrete formalisation. This is not an inscription of motion but the generation of signals being fed into circuits and interfaced with an analogue output. And computers are operating independently from human beings according to protocols of calculation and address-management based on a coding that is self-referential. Computers may - in other words receive signals and process them, yet they do not compute human beings and the world but - in mercilessly flawless recursions - only themselves.

It is thus not motion captured in sound or codings, but motion disassembled into discrete parameters, which are transcoded to fit the unity of a dataset consisting of zero and one. This data, relayed to units of sound, writing, image, constitutes the output. Mapping is a process quite different from recursion within the autonomy of coding, as it takes place in computers on the level of symbolic calculation. Mapping is trans-coding and thus representation or, differently put, it already has and always will happen in the realm of representation.

\section{Imitation and Interaction: \\ Electrohysteria and the Camouflage of Technology}

The camouflages, suppressions and deceptions practised in these performances are strategies of reconfiguring computers as something they have technically never been. I would argue this reconfiguration of technology as the prerequisite for the association of humans and machines with the Resonance Paradigm around 1900.

To imply that computers and discrete codings are in any way connected to human beings and motion is clearly the product of a discursive inscription,

\section{0}


which may draw on the still resonant insanity of resonance between man and media around 1900. The implied recursion ${ }^{32}$ turns out to be camouflage, coverage of technology, which in turn allows the discursive production of computers as media by means of aesthetic-dramaturgical practices. The two strategies used in this context are (1) interaction and (2) imitation.

Covering technology creates the (1) aesthetic impression of interaction and the impression of flow is crucial in this context. More than anything it is flow, the staged immediacy of a relation between dance and music, that would turn computers towards human beings, by developing and training strategies which transcribe discrete operations into continuous phenomena and perceptions. This kind of imaginary interaction with computers not only blocks its intrinsically time-critical dimension, but also purges constituent elements of digital encoding such as the inclusion of defects from the scenario of signal transport.

This configuration of interaction is finally staged as a resonance between performers and computers by summoning iconographies whose power derives from their traditional embedding in cultural memory. This is achieved by a simple strategy of technical and aesthetic imitation, since the aesthetic design, the output of images and sound imitates (2) the phenomena inscribed or made visible by electro-electronic devices: waveforms, frequencies, distorted sounds, electro-acoustic tonalities, and multiplications of images. This is to say, that these manipulations refer to technologies belonging to the realm of the electro-electronic; they may be generated based on the deflection of electrons and sound frequencies. The analogue surfaces of computers, like screens or - as far as the conversion of analogue data is concerned - the MIDI converter, are used to imitate these manifestations of the electrico-electronic. Performance with computers thus seems to have made little progress since Nam June Paik's practice of an "aesthetics of deflected electrons" 33 . Needless to say, this aesthetics of the electron obscures its sources, operates beneath the surfaces, under the cover of camouflages, and technically speaking is based on discrete coding. It does entail, however, a cultural, electro-electronic re-programming of computers, whose technological sedimentation and implementation may even be observed in devices like MIDI interfaces, sensor interfaces and software environments like Max/MSP/Jitter. Manipulation of signal chains and circuits themselves, however, does not occur at all, or remains the exception.

32 More precise in terms of technology, we must speak of re-coding. If there were recursion, motion would write itself acoustically without mediation. Yet this only happens, if motion is already available as relay-able, discrete symbolic coding. Motion itself, however, cannot discretely be addressed "directly".

33 Leeker (in print) 
Interaction and the imitation of the electro-electronic superimposes the inputs of human beings and machines and thus computers are generated as a continuation of the kind of electro-electronic epistemology of around 1900, described earlier. Where computers are used and thus a technological transformation of flowing energy in digital codings and rhythms of information occurs, these very structures and events are covered. At the same time, the conversion of analogue data into discrete data that separates human beings from machines is buried under this cover. ${ }^{34}$ Call it a tactical misapprehension of computers' discrete "nature".

\section{Interactive Performance with \\ Computers, Electro-hysteria and Cybernetics}

Interactive performances with computers since the $1960 \mathrm{~s}^{35}$ have thus managed to (re)create the computer from nothing but the epistemology of the electro-physiological experimental settings around 1900. This in turn brings forth cybernetic computing whose "merit" it has been to induce an understanding of human beings as information processing machines and to have liquidated the computer as a symbolic machine.

The crucial aspect of this connection is the inclusion of the human organism in the image of a system controlled by electrical currents and circuits that designs this organism as a control device for integration in cybernetic feedback loops. ${ }^{36}$ The electro-hysteric-cybernetic analogy of human beings and computers created by performances are thus of particular productivity. They merge both entities to one system, which is to say, that human beings are now part of an autonomous feedback loop of discrete operations - a prerequisite for these two systems to form an interactive relationship at all. Performances thus realise the integrated cybernetic feedback loop for human beings and universal machines, although the latter operate based on invariant chains of symbols.

\section{Media Technologies: \\ Towards a History of their Dramaturgy}

The history of philosophy and the history of knowledge prepared an epistemological field, which allowed and allows devices, machines and cultural techniques to turn into media. Media emerged and emerge from discursive and cultural "programming" ${ }^{37}$, which may crystallise in technologies, as

\section{Hagen 2002a}

35 The connection of computers and performance started with the experiments of the artists group EAT at New York during the 1960s. See Büscher (1998).

36 Büscher 1998

37 Schröter 2004, pp. 8-15

\section{2}


demonstrated in the instance of the MIDI interface. Performative techniques, dramaturgies, perhaps the instance of play as such, perception and camouflage are central to this process.

For Media Studies, this may well mean that they must always deal with the performative and that they have to do so in very specific and precise terms. After all, the instances discussed in this paper show that theatrical practices like camouflage, deception, empowerment, mimicry and imitation have been practices of immense power. They managed to reconfigure the computer against its own technological history and thus bestowed it with the potential to create worlds accessible for human beings.

For Theatre and Cultural Studies, these findings suggest approaches that seek proximity to the analysis of technical materialities, since this may well be the only way to retrieve and decipher the discourses and the cultural programming of media technologies - including those by one's own scholarly discipline.

There is little doubt that the interaction with computers in performances is a valid field of research in this context, since it refers to both foundations and training within a historical account of the dramaturgy of media technologies. The Resonance Paradigm is a cornerstone of this history, not merely because it continues to resonate in the interaction with computers up to this very day, but also as a cultural technique generating media as well as modes of using them.

Translated by Michael Barchet

\section{References}

Bahr, Petra (1999): »Loie Fuller. Grenzgängerin des Tanzästhetischen (1)«. Ta katoptridsomena. Magazin für Theologie und Ästhetik 2. Online available: <http://www.theomag.de/02/pb1.htm> (last access: October 2007).

blackhole-factory (2001): »Feedback«. Online available: <http: / www.blackhole-factory. de/feedback.html> (last access: October 2007).

Böhnke, Alexander/Schröter, Jens (2004): Analog/Digital - Opposition oder Kontinuum? Bielefeld: transcript.

Büscher, Barbara (1998): »InterMedia-Material«. In: Gabriele Brandsteter/Helga Finter/ Markus Weßendorf (Eds.), Grenzgänge. Das Theater und die anderen Künste, Tübingen: Gunter Narr Verlag, 113-125.

Crookes, William (1897): »Address by the President«. In: Proceedings of the Society for Psychical Research, Volume XII, London: 338-355. 
Didi-Huberman, Georges (2004): The Invention of Hysteria: Charcot and the Photographic Iconography of the Salpetriere, Cambridge: MIT Press (Orig. French 1982).

Hagen, Wolfgang (1995/96): »Theorien des Radios. Ästhetik und Äther«. Online available: <http://www.whagen.de/seminare/AETHER/Aether 1.htm> (last access: October 2007).

Hagen, Wolfgang (2001): Radio Schreber. Der 'moderne Spiritismus' und die Sprache der Medien, Weimar: Verlag und Datenbank für Geisteswissenschaften. Online available: <http: / / www.whagen.de/publications / RadioSchreber/radioschreber. htm> (last access: October 2007).

Hagen, Wolfgang (2002a): »Camouflage der Kybernetik«. Online available: <http: / /www. whagen.de/vortraege/Camouflage/CamouflageVortrag.htm> (last access: October 2007).

Hagen, Wolfgang (2002b): »Die entwendete Elektrizität. Zur medialen Genealogie des 'modernen Spiritismus'«. Online available: <http://www.whagen.de/publications/ EntwendeteElektrizitaet/26Hagen.htm> (last access: October 2007).

Hagen, Wolfgang (2005): Das Radio. Zur Geschichte und Theorie des Hörfunks Deutschland/USA, München: Wilhelm Fink Verlag.

Helmholtz, Hermann von (1954): On the Sensations of Tone as a Physiological Basis for the Theory of Music, New York: Dover (Orig. German 1863).

Herrmann, Hans-Christian von (1996): »Fantaskopie. Zur Technik des Blicks im Kino«. Stroboskop. Die Zersplitterung des festen Blickpunktes ( = Kaleidoskopien. Vol. 1, Leipzig, 1996), 17-23.

Herrmann, Hans-Christian von (2005): Archiv der Bühne, München: Wilhelm Fink Verlag.

Kittler, Friedrich A. (1980): Austreibung des Geistes aus den Geisteswissenschaften. Programme des Poststrukturalismus, Paderborn: Schöningh.

Kittler, Friedrich A. (1985): Aufschreibesysteme 1800/1900, München: Wilhelm Fink Verlag.

Kittler, Friedrich A. (1992): Discourse Networks, 1800/1900, Stanford: Stanford University Press.

Krämer, Sybille (1998): »Sprache - Stimme - Schrift. Sieben Thesen über Performativität als Medialität«. In: Erika Fischer-Lichte/Doris Kolesch (Eds.), Kulturen des Performativen ( = Paragrana 7/1), 33-57.

Krämer, Sybille/Stahlhut, Marco (2001): »Das 'Performative' als Thema der Sprachund Kulturphilosophie«. In: Erika Fischer-Lichte/Christoph Wulf (Eds.), Theorien des Performativen ( = Paragrana 10/1), 35-64.

Leeker, Martina (2005): »Digitale Operativität und Performance. Geschichte der MenschComputer-Schnittstelle im Moment ihrer Hinterfragung, noch bevor sie anfing «. In: Klaus-Peter Köpping/Bettina Papenburg/Christoph Wulf (Eds.), Körpermaschinen Maschinenkörper. Mediale Transformationen ( = Paragrana 14/2), 25-51.

Leeker, Martina (2008): „Camouflagen des Computers. McLuhan und die NeoAvantgarden der 1960er Jahre«. In: Derrick de Kerckhove/Martina Leeker/Kerstin

\section{4}


Schmidt (Eds.), McLuhan neu lesen. Medien und Kultur im 21. Jahrhundert, Bielefeld: transcript, 345-375.

Leeker, Martina (in print): »Störungen und Erkenntnistrübungen in Maschinentheatern. Kulturelle Leistungen bildgebender Oberflächen (quadro) im 17. Jahrhundert und seit den 1960er Jahren«. In: Helmar Schramm et al. (Eds.), Spuren der Avantgarde. Theatrum Machinarum, Berlin: de Gruyter.

Pias, Claus (Ed.) (2003): Cybernetics/Kybernetik. Die Macy-Konferenzen 1946-1953. Vol. 1, Berlin: diaphanes.

Pias, Claus (2004): »Elektronenhirn und verbotene Zone. Zur kybernetischen Ökonomie des Digitalen«. Online available: <http:/ /www.uni-essen.de/ bj0063/texte/elektronenhirn.pdf> (last access: October 2007).

Schrenck-Notzing, Albert von (1904): Die Traumtänzerin Magdeleine G. Eine psychologische Studie über Hypnose und dramatische Kunst, Stuttgart: Ferdinand Enke.

Schröter, Jens (2004): Das Netz und die virtuelle Realität. Zur Selbstprogrammierung der Gesellschaft durch die universelle Maschine, Bielefeld: transcript.

Siegert, Bernhard (2006a): »Spectres. Faradays Experimente 1830-31«. In: Daniel Genthmann/Christoph B. Schulz (Eds.), Apparaturen bewegter Bilder, Münster: Lit, 36-50.

Siegert, Bernhard (2006b): »Radio Art um 1900: Crookes, Peirce, Lodge und Duchamp«. Online available: <http://www.uni-weimar.de/medien/kulturtechniken/lehre/ ws2006/material/VLRadio04-Netzversion.pdf> (last access: October 2007).

Siegert, Bernhard (2006c): »Funken und Strahlen: Zum Okkultismus der Moderne (Hertz, Crookes, Peirce, Branly, Lodge)«. Online available: <http://www.uni-weimar. $\mathrm{de} /$ medien/kulturtechniken/lehre/ws2006/material/VLRadio03-Netzversion.pdf> (last access: October 2007).

Volmar, Axel (2003): »Parametrisierungsgeschichte der neuzeitlichen Akustik«. Online available: <http://www.aesthetik.hu-berlin.de/medien/texte/vol_para.pdf> (last access: October 2007).

Wellberry, David (1992): »Foreword to Friedrich Kittler, Discourse Networks, 1800/1900«. In: Friedrich A. Kittler (Ed.), Discourse Networks, 1800/1900, Stanford: Stanford University Press, XI-XVI.

Winkler, Hartmut (2004): »Performativität«. In: Hartmut Winkler, Diskursökonomie. Versuch über die innere Ökonomie der Medien, Frankfurt a.M.: Suhrkamp. Online available: <http://wwwcs.uni-paderborn.de/ winkler/d-oek-12.pdf> (last access: October 2007). 


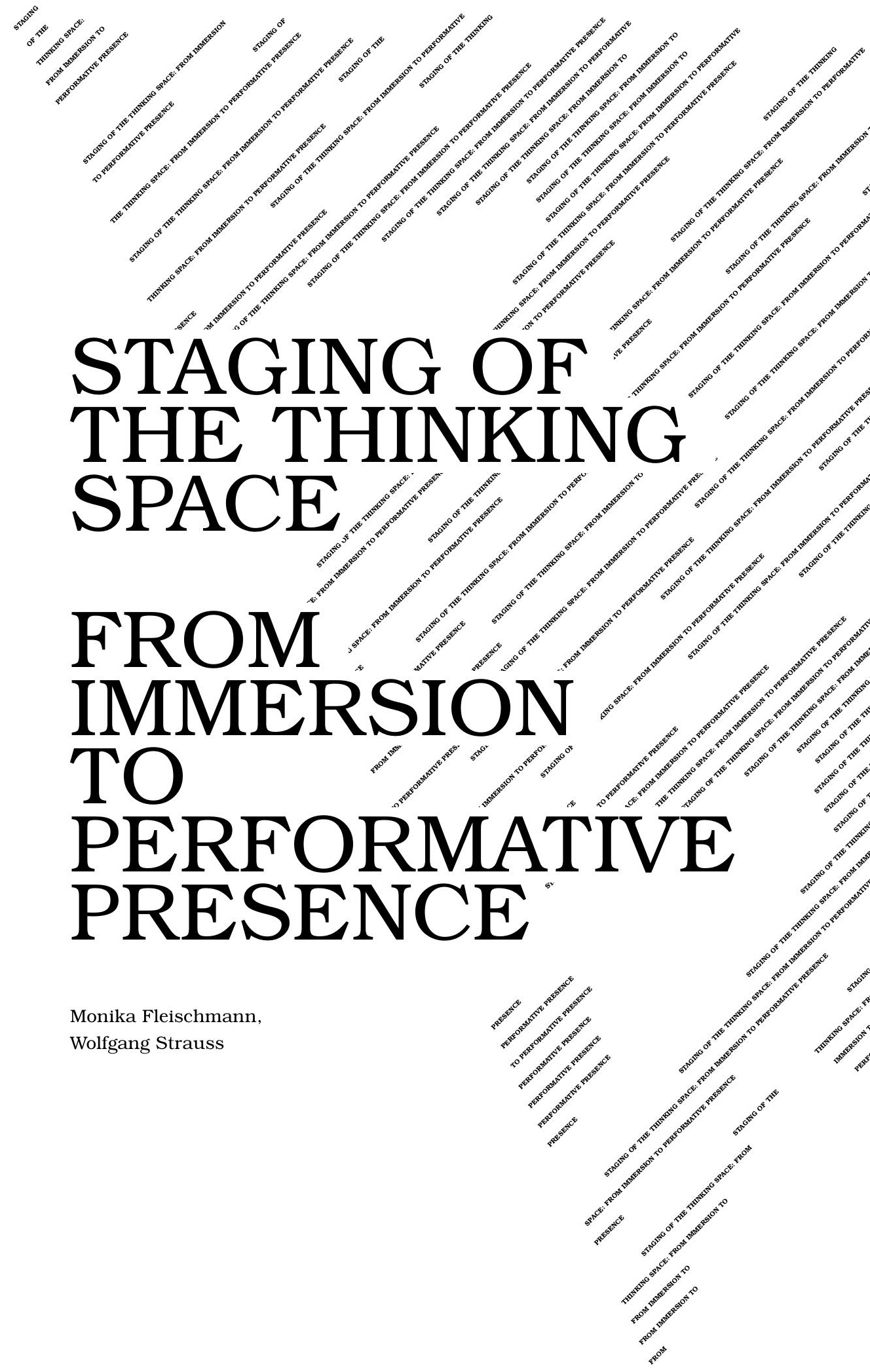




\section{Introduction}

The term "media art" will be used here for artistic activity which either uses or schematises digital technology interactively. Media art begins where the traditional film genre ends: with interactive and process-related digital narratives. The cinema builds up a relationship between audience and presentation, and the viewer is promised a collective experience. By contrast, interactive storytelling offers the observer an individually tailored encounter, where the YOU_ser ${ }^{1}$ is navigating the story. Based on rules of staging, media art conjoins with other cultural forms of expression such as performance, dance, theatre, film, architecture, sound, design or fashion. Media art gets its poetic strength from the new possibilities of interacting with its audience and from the cross-over between cognitive science ${ }^{2}$ and arts. In the last two decades, the authors have carried out media art research in institutes like $\mathrm{Art}+\mathrm{Com}^{3}$ in Berlin, the Academy of Media Arts in Cologne and currently the Fraunhofer Institute IAIS near Bonn.

Up until today, the use and maintenance of media art has been technically challenging. Hardware and software are changing constantly, and the works of art would have been adapted for every new $\mathrm{ICT}^{4}$ generation. Therefore many works of media art from the 80s and 90s can no longer be seen. If not restored by the artist, the works are unlikely to be restored by a museum or a gallery, for financial reasons or because of lack of competence. Nevertheless, no other art form is so close to our present day electronic world, and it therefore deserves greater attention. Interactivity, narrativity and digital scenographic audiovisual production are important topics of new media art. In contrast to cinema, interactive media art orchestrates the senses e.g. by staging tactile elements - even virtual ones - on a virtual stage next to visuals and acoustics. Digital storage and statistical procedures make visible what would otherwise remain invisible. Participation of the spectator in processing interactive media art creates awareness and new knowledge (Erkenntnis). Our thesis is that interactivity supports performative communication and the creation of performative presence.

1 For interactive media art at ZKM exhibition "YOU_ser" see <http://on 1.zkm.de/ $\mathrm{zkm} /$ stories/storyReader\$5591>.

2 Cognitive science is defined as the study of mind or of intelligence. The interdisciplinary study draws on relevant fields including psychology, psychiatry, philosophy, neuroscience, linguistics, anthropology, computer science, and biology. The term was coined by Christopher Longuet-Higgins in 1973 concerning the then-current state of Artificial Intelligence research. See <http://en.wikipedia.org/wiki/Cognitive_science>.

3 In 1988, a group of architects, artists, designers, scientists and technicians, amongst them Monika Fleischmann, Wolfgang Strauss, under the direction of architect Edouard Bannwart, co-founded the collective Art+Com e.V., researching and developing information design and communication technology.

4 ICT - Information and Communication Technologies

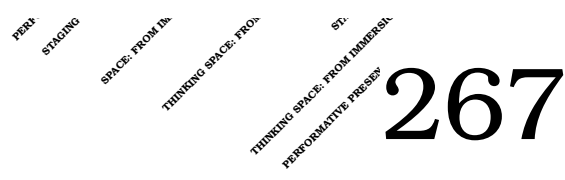




\section{Interactive Media Art as Performative Communication}

In the 1980s and 90s, media artists experimented between science and art with the aesthetic potential of process-related image genres. Technological institutes, namely in Germany, the US and Japan, invited artists as researchers. The art historian Oliver Grau (2004) writes: "Internationally renowned exponents such as Charlotte Davies, Christa Sommerer and Laurent Mignonneau, Monika Fleischmann and Wolfgang Strauss, Jeffrey Shaw or Victoria Vesna, work, as a rule, as scientists in research laboratories and, for example, develop new interfaces, interaction models and code innovations. So they set anew the technical limits according to their aesthetic aims and critical messages." Experimentation stands at the forefront of the new telepresence. Via broadband, the Virtual Environment can be accessed worldwide.

In autumn 1991, the authors sent an early version of the Virtual Reality ${ }^{5}$ installation "Home of the Brain" ${ }^{6}$ over the ISDN data lines from Art+Com in Berlin to the exhibition space in Geneva. Visitors in Geneva moved with a data glove through a virtual Potsdamer Platz and the virtual New National Gallery. "Home of the Brain", a virtual exhibition and communication environment, translated the antique Stoa concept of a public place for meeting and discussion into the virtual space. The visitor navigates with a data glove through virtual rooms that are made visible with data glasses. Hand movements activate the citations of four media thinkers who play an important role in the theoretical formation of media culture. They are represented alongside their theoretical concepts by individual thought buildings, literally speaking houses dedicated to Joseph Weizenbaum, Marvin Minsky, Paul Virilio and Vilém Flusser. At the end of the 1980s, the work was designed to reflect the current media discourse.

"Home of the Brain" is staged as an encounter in areas of thought - a Virtual Denkraum. The visitor is part of the staging and becomes located in the minds of others. The current discourse is reflected through the medium itself. The virtual space is interwoven with light, shade, colour, texture, words and sound, and all together with movement. The art historian Oliver Grau describes the Virtual Reality installation as media theory put into practice, and a new mnemonic theatre that anticipated the form of communication with networks: "'Home of the Brain' therefore emerges as early as 1991 as an early appearance of the epistemic innovation telepresence. As a conse-

5 With the term "virtual reality", communications technology has offered equipment and concepts which allow an entry into the virtual environment. Data gloves and data glasses bring the observer into the setting, as the authors show with "Home of the Brain". See <http: / www.medienkunstnetz.de/works/home-of-the-brain/>.

6 Fleischmann/Strauss (Art+Com) 1990-1992

\section{8}


quence, the reception of the art work in this way loses its local fixation, the observer does not go to the work, to the panel, panorama, cinema film etc., however, the work does not come exclusively to the observer." 7

\section{Origins of Virtual Reality}

When we started our research in 1988 , there was a virtual void. We were thinking about computational models. We were looking for the underlying structure, for navigation and orientation in virtual space. There was a need for new paradigms of space and interactivity. How should we deal with telepresence? How should we organise information? How should we link information and interaction with virtual objects? We studied our material and looked for adequate methods. It was "like studying celluloid instead of cinema, paper instead of novels, cathode ray tubes instead of television, hardware instead of software." And we concentrated on the interface to explore the role of the senses in mixed reality space.

Virtual Reality evolved from mechanical simulators for the training of combat pilots in the Second World War and computer graphics research in the early 60s. "Virtual Art" , based on Virtual Reality (VR), the making of interactive environments, had nearly no resources in the traditional art world to have recourse to. In "The Ultimate Display", computer scientist Ivan Sutherland (1965) published the theoretical foundations for VR: "The ultimate display would, of course, be a room within which the computer can control the existence of matter. A chair

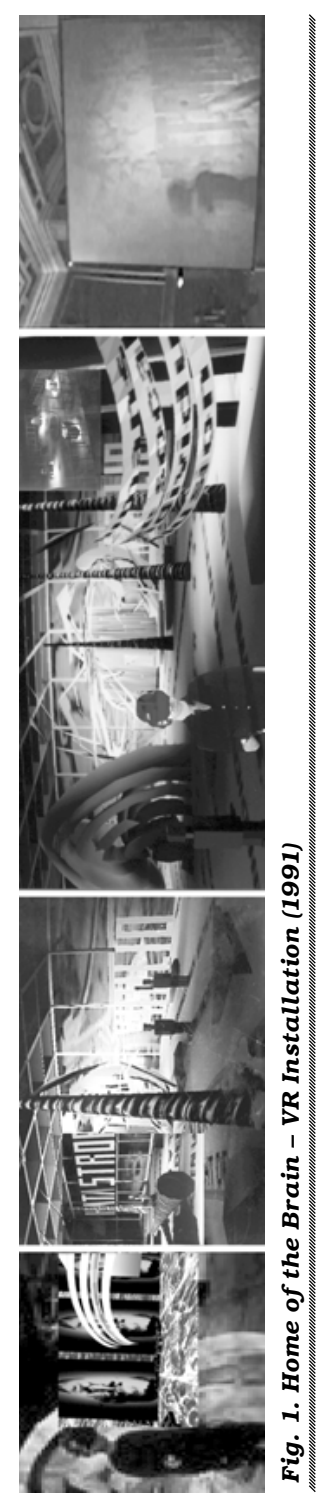

7 Grau 2004

8 Bates 1991

9 For the history of Virtual Art see Grau 2003. 
displayed in such a room would be good enough to sit in. Handcuffs displayed in such a room would be confining, and a bullet displayed in such a room would be fatal. With appropriate programming, such a display could literally be the Wonderland into which Alice walked." There was the idea of a computer-based interactive fantasy system to "go anywhere and do anything. With goggles and gloves the interface hardware and software problems were solved by Scott Fisher in 1986 at NASA Ames Research and Frederick Brooks in 1988 at the University of California. ${ }^{10}$ In our research we used VR as a medium to express an idea, a vision of future communication or about the future city after the Berlin Wall had fallen. The authors' interactive table installation "Berlin, Cyber City"11 (1989-90) was the origin of the tabletop interface "Responsive Workbench"12 (1993-94).

\section{Space: From Performance Space to Performative Presence}

Where did inspiration come from? At first one described only such 3D real-time simulations as Virtual Reality, but then in the 1990s the internet itself was linked to William Gibson's term 'cyberspace' (from cybernetics and space). ${ }^{13}$ With "Johnny Mnemonic" from the short story collection "Cyberspace", and with his novel "Neuromancer" from 1984, Gibson revolutionised the way people look at technology. But inspiration came not only from technology research, computer graphics and science fiction. Film, theatre, music, literature and pop culture also had an influence on the development of interactive media, virtual art and the creation of transformative spaces. ${ }^{14}$ Virtual Reality is the fusion of real-time, space, interface, haptics and movement with image and sound in conjunction with mathematical thinking. In our artistic work we are interested in the perception and reflexion of the current situation of communication technologies. Since 1988 we have been working on cultural interfaces to link real and virtual space. We initiated our

10 For an "American" Timeline of Virtual Reality see <http: / chrishutchison.org/atticaschool/vr/Issues / page31/page31.html>.

11 "Berlin, Cyber City" was invented by the authors with a team at Art+Com as an interactive table installation with the virtual reconstruction of the city of Berlin. See <http: / / netzspannung.org/database/cyber-city/en>.

12 The prototype of the "Responsive Workbench" was invented by the authors under the direction of Wolfgang Krueger and with the team at GMD - the German National Research Centre for IT. See <http: / / netzspannung.org/database/responsive-workbench/en>.

13 For the term "Cyberspace" see on Wikipedia <http://en.wikipedia.org/wiki/ Cyberspace> and ARTE Portrait 2005: Dream - William Gibson. See <http://www. arte-tv.com/de/kunst-musik/tracks /Diese-Woche/20050203/804294.html> (last access: June 2008).

14 Packer/Jordan 2002

\section{0}


own Labs ${ }^{15}$ to study and produce the interlinking of art, technology and science. In transdisciplinary teams we find the patterns that make a difference by using the epistemologies of each discipline to drive inquiry. We take theories and methods which exist independently of several disciplines and apply them to organise and understand different areas for the purpose of achieving new insights.

The VR installation "Home of the Brain" relates to concepts of the avantgarde theatre, such as Samuel Beckett's "Quadrat", where people react to one another and build up relationships by walking on predefined paths. We were inspired by the late Klaus Michael Grueber, the wanderer among the stage directors, who used unusual public spaces to appeal to the audience. The theatrical performance installation "Rudi" (1979) dealt with forms of remembering. The setting, near the - at that time still existing - Berlin Wall, in the prestigious Hotel Esplanade ${ }^{16}$, became a stage and exhibition space for an outstanding performance. It involved a tour through the house that sets thoughts into motion, although hardly anything in the space moves. An actor sits in front of a fireside and read out Bernard von Brentano's 1934 novel "Rudi" in a monotonous voice and with many breaks - loudly. Parts of his reading were transmitted into the other rooms of the hotel through loudspeakers. The audience moved around as if in an exhibition in order to incorporate the idea of spatially performed information related to the voice, movement and stage. There was no linear narrative structure to follow. It was not clear: Is this reality, theatre or a museum? The borders of real and fictional space blurred and became one (mixed) reality. The audience was part of the concept of an aesthetic experience. It impressed and unsettled the people of Berlin in the ruins near Potsdamer Platz. The merging of theatre and museum in "Rudi" transferred the visitors into a situation of in-betweenness. ${ }^{17}$ The VR environments "Home of the Brain" or the subsequent "Murmuring Fields" 18 refer to fundamentally different notions of space: the performative presence of becoming in the virtual space and the physical presence in the space of performance. ${ }^{19}$ During the press conference where "Home of the Brain" was presented with the Golden Nica of the Prix Ars Electronica in 1992 in Linz a visitor explored the VR environment. He moved his whole body as if he were

15 First Art+Com, later the Fraunhofer MARS Exploratory Media Lab. See <http:// netzspannung.org/about/mars/projects/en>.

16 The famous Hotel was damaged in the Second World War, but still became the scene of famous films. Today, the preserved parts of Hotel Esplanade can be visited in the Sony-Center at the Potsdamer Platz, where they were transferred in 1996 by means of complicated technology. See <http://de.wikipedia.org/wiki/Hotel_Esplanade_(Berlin)>.

17 Fischer-Lichte 2008

18 Fleischmann/Strauss 1997-99

19 Gemeinböck 2004 
swimming around the virtual objects. He represented an in-betweenness of being in both spaces - the real and the virtual.

\section{Time: Film as Seismograph of Nonlinear Image and Sound Concepts}

Experimenting with Virtual Reality in the 80 s and 90s, artists and scientists felt still influenced by the Apollo space-flight program undertaken by NASA during the years 1961 - 1975. They were affected by films such as "2001: A Space Odyssey" or "Powers of Ten" presuming the non-linearity and the endlessness of space that was first experienced with Virtual Reality technologies - but then interactive and in realtime. "2001: A Space Odyssey" by Stanley Kubrick ${ }^{20}$ is a science fiction epic and was flavour of the moment in 1968: America was in space fever. The film is not only a future vision about contact with aliens and the endlessness of space, but also about the questions of life that are often interpreted by artists and philosophers: "Where Do We Come From? What Are We? Where Are We Going?"21 In an interview Kubrick said that he "tried to create a visual experience, one that bypasses verbalized pigeonholing and directly penetrates the subconscious with an emotional and philosophic content." ${ }^{22}$ Instead of using much dialogue, ${ }^{23}$ Kubrick achieved his goal by using music and sound atmospheres essentially by Gyorgy Ligeti, Richard Strauss and Johann Strauß. The film begins with a black image and Ligeti's "Atmosphères" (1961). In this piece melody and rhythm are blurred beyond recognition through the creation of sound complexes. It concentrates on the texture of the sound. The work is, as Ligeti said, "just a floating, fluctuating sound, (....) You hear a kind of impenetrable texture, something like a very densely woven cobweb." ${ }^{24}$ Ligeti's works create the suitably weird sound effects for the more "far out" trips of the film. ${ }^{25}$ A feeling of hallucination was created, only with sound and images. ${ }^{26}$ Critics characterise the film as being

20 Interview with Stanley Kubrick "2001: The space odyssey" explaining a treatment of the film can be found on the internet by New Media Giants. See <http: / /www. kubrick2001.com/> (last access: June2008).

21 This was the title of Paul Gauguin's philosophical painting from 1897/98.

22 Stanley Kubrick by Eric Norden. Playboy Magazine. September 1968

23 There are only $43 \mathrm{~min}$. in this film of $143 \mathrm{~min}$. length.

24 For Program Notes of San Francisco Symphony see György Sándor Ligeti, San Francisco Polyphony. See <http://www.sfsymphony.org/music/ProgramNotes.aspx?id $=28950>$.

25 György Sándor Ligeti, San Francisco Polyphony. See <http: / / www.sfsymphony.org/ music $/$ ProgramNotes.aspx?id=28950>.

26 Kubrick used more than $33 \mathrm{~min}$. of Ligeti's "cluster sound". Though he did it without the composer's knowledge or permission, the film created a constantly growing international interest in Ligeti's music, not only in the classical but also in the popular world.

\section{2}


"hypnotically entertaining, and it is funny without once being gaggy, but it is also rather harrowing." 27 The film's well-known fanfare and title music, "Also Sprach Zarathustra, Op. 30" is a tone poem by Richard Strauss, composed in 1896 and inspired by Friedrich Nietzsche's book of the same name from $1883 / 1885$. The title sequence begins after the black image with the Earth rising over the Moon, while the Sun rises over the Earth. "Thus Spoke Zarathustra" was used in similar situations such as the TV coverage of the Apollo Moon missions and landings in the late 1960s and 1970s. For the space scenes Kubrick filled the vacuum of space paradoxically with "The Blue Danube", the famous Waltz by Johann Strauss Jr.: "it certain[ly] suggests the dance of space craft under the slow inexorable influence of Newtonian gravity and mechanics. The Space Station pirouettes, while inside a member of the cabin crew demonstrates walking under zero gravity conditions while objects like pens float off." 28 Although the film does not adhere to the audience's usual expectations - there is no action, no plot and no resolution - "2001" became one of the most successful films ever and a classic of cinema history. Alongside the lack of a plot, the characteristic of boundless und bottomless floating in space is similar to the sense of space in Virtual Reality, and the structure of Ligeti's music is reminiscent of interwoven algorithms.

Another example of seismographic, self-proclaiming new image concepts is the nine-minute film structured as a documentary, "Powers of Ten" by Charles and Ray Eames, which likewise arose in 1968 within the context of space research. It demonstrates zooming and scaling - further design principles of interactive media. The film takes the audience on a journey, which begins with a picnic on the seashore in Chicago and leads to the edge of the universe. Every ten seconds we can see the starting point of the journey from a distance ten times further away from the earth, which then can only be seen as a point of light between many others. On the return journey, the view is enlarged, with breathtaking speed, tenfold every ten seconds. The camera shows the sleeping picnicker, then reduced to the view of his hand, then into his hand, and ends up on the inside of the cells of one of his DNA molecules. Charles and Ray Eames, with this film, give an idea of the relative size of objects in the universe. They show how a single idea can reflect a universe of thoughts. Questions of measurement shape our understanding of the world. Knowledge of measurement and scale - as shown here - change the perspective of all things. Both films from 1968 are very much supported by atmospheric sound. In both, the earth is seen from space and here it was seen for the first time as a vulnerable global home worthy of protection. This perspective gave us a feeling for our place in the universe.

27 Gilliatt 1970

28 "2001: A Space Odyssey - Original Soundtrack" online available <http://www. mfiles.co.uk/reviews / 2001 -a-space-odyssey.htm> 


\section{Locative Media for presenting the World}

Locative Media is a new form of land art, where artists reflect e.g. on surveillance as a new form of presence. Networked Nature, or Locative Media, has a history that predates that first satellite launch in 1978. Bleecker and Knowlton (2006) write about the origins of GPS-Enabled Locative Media: "For instance, the 'Earthworks' group exhibition in October of 1968 in New York may count as a canonical point in the history of such geography and land form inspired art works. There is a distinction to be made of motivation as well as technique, which is what we mean to draw out by demarcating presatellite from satellite-enabled locative media. (...) It is painfully ironic that, in a time when public funding for art in the U.S. has evaporated, locative media artists are able to "piggy-back" on the U.S. Department of Defense, in a fashion, appropriating GPS technology for creative purposes. (...) Consequential financial, political and creative-capital investments are one of the drivers of interest in the digital territorialization of physical geography, thereby establishing it as an interface for electronic media experiences. Through this territorialization, real-estate has become virtual-estate." ${ }^{29}$

In 1996 Art+Com presented TerraVision ${ }^{30}$, a self-contained, Virtual Reality, 1-to-1 representation of the Earth. A stylised globe was the interface for the audience to zoom in on any location in the world and obtain minutely detailed pictures. Ten years later this kind of system was online: In July 2005, the search engine Google, with "Earth", created the possibility of a virtual world tour by satellite picture, with zoom function. Chip Online wrote: "The digital globe makes zooming from space to home town possible." ${ }^{31}$ In this way, the filmic archetype "Powers of Ten" is now directly available for every computer user. The online globe offers a 3D map of many parts of the earth, in that it compiles satellite and aerial photography of towns and industrial areas. The victims of the hurricanes in New Orleans and elsewhere used "Google Earth" in order to form a picture of the destruction of their homes and neighbourhoods. "Google Earth" picks up on the idea of scaling, in order - it appears to get to grips with the world: regarding its weather systems, its economy and global totality. Satellite images send views from a great distance and create a distanced point of observation. These images, which seemingly extend our sight limitlessly, suggest definitions such as: total surveillance, total overview. These are global positioning systems of complete visibility of time and space.

\section{Bleecker/Knowlton 2006}

30 The project was started by Uli Weinberg at Terratools. See <http: / / www.artcom.de/ index.php?lang=en\&option $=$ com_acprojects\&id $=5 \&$ Itemid $=144 \&$ page $=6>$.

31 More about Google Earth in "Chip Online de" see < http: / / www.chip.de/downloads / c1_downloads_13015193.html>.

\section{4}




\section{Presence: Models for Interactivity}

The idea behind the narrative form "film" is to represent events and tell stories arising from researching the realms of the psyche. Where film ends, the digital and interactive, process-related environment begins. Media artist Simon Penny (1995) differentiates the difference in perception of a painting, a film and of interactive media art as follows: "A painting is an instance of representation. A film is a sequence of representations. Interactive artworks are not instances of representation, they are virtual machines which themselves produce instances of representation based on real time inputs."

The theatre, with its abstracting stage and real-time input, appears a better role model for interactive concepts than film, with its ready-made images. "Home of the Brain" was conceived as a digital memory space and as a venue for battles of spoken words. The theatrical paradigm stands for the positioning of information in space and for animating the audience. Nonetheless, visitors understand their virtual observer perspective in "Home of the Brain" as if they were walking through a film. This is because this virtual surrounding could only be experienced as an individual with data glasses and data gloves.

The return channel for the virtual meeting with others was technically only realisable a few years later with "Murmuring Fields"32 (1998-2000), an audiovisual soundspace for several interactors on stage. We had built a shared environment for real-time interactive performance. So the virtual space became not only metaphorically virtual, but also physically real, an accessible and tangible sound. For "Murmuring Fields" we developed a mixed reality ${ }^{33}$ method for the penetration and superimposition of physical and electronic space. Data space and stage space are interconnected with one another and overlap by means of an optical tracking system. Two dancers utilise the interactive sound space of different sites. With their bodies in action, they employ the soundspace as an instrument. They play with words, images and sounds, and they oppose the system with their bodies in order to avoid being overwhelmed by technology.

In "Murmuring Fields" digital information - sounds and figures - is located in the space as if the room were furnished with data. ${ }^{34}$ Every movement of

32 Murmuring Fields is documented on the online media art archive netzspannung. org <http: / / netzspannung.org/database/murmuring-fields> and on MedienKunstNetz <http: / /www.medienkunstnetz.de/works/murmuring-fields>.

33 Mixed reality (MR) (encompassing both augmented reality and augmented virtuality) refers to the merging of real and virtual worlds to produce new environments and visualisations where physical and digital objects co-exist and interact in real time. See <http: / / en.wikipedia.org/wiki/Mixed_reality>.

34 Strauss 1999 


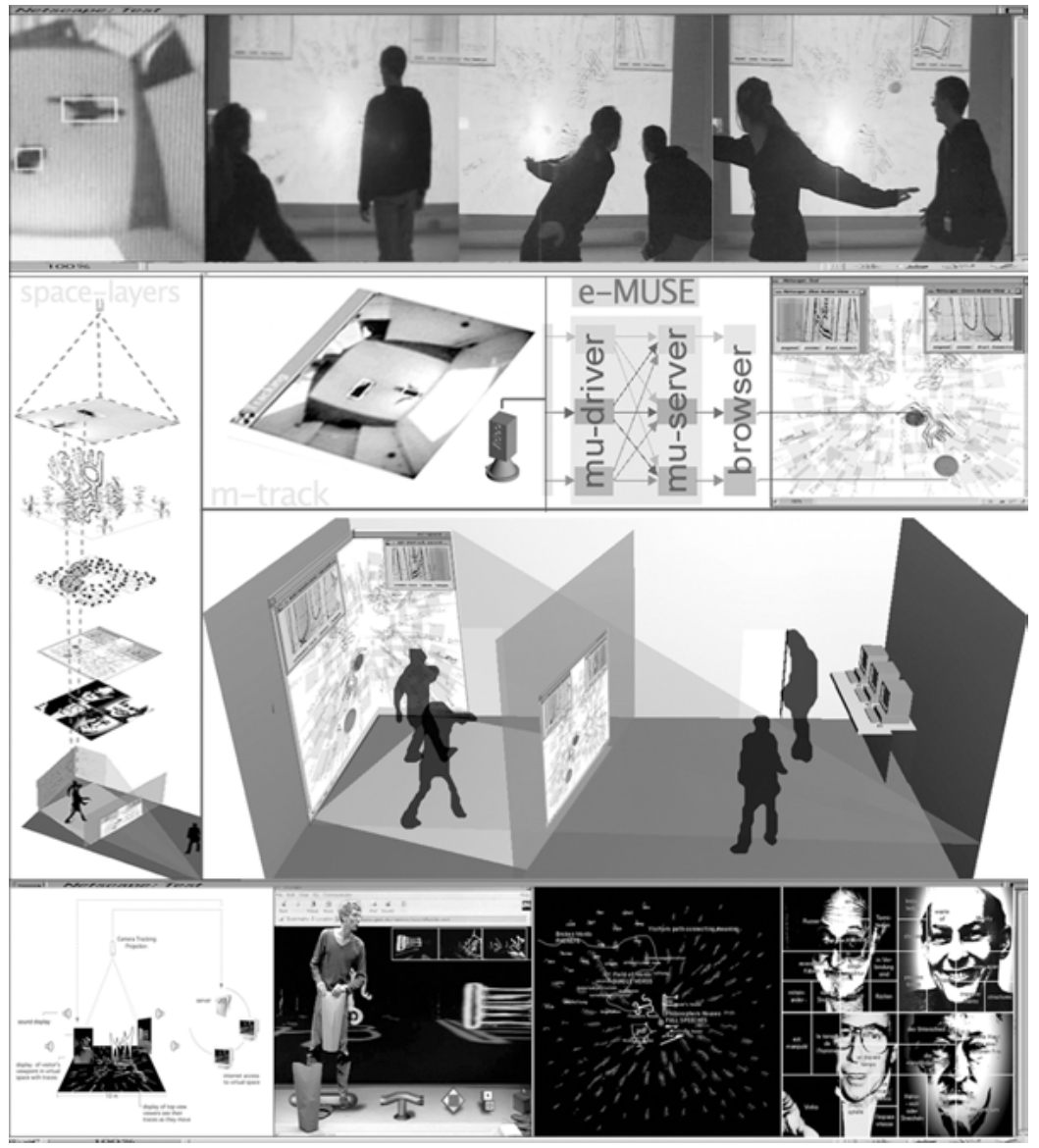

Fig. 2. Murmuring Fields - electronic multi user stage environment (1991)

the body is captured with an optical body-tracking technique. ${ }^{35}$ Movement is transferred from real space into data space, and translated into a sound collage. Spoken texts are broken up into words and syllables. Movement in space creates movement in the text. Two interactors produce text samples by Joseph Weizenbaum, Marvin Minsky, Vilém Flusser and Paul Virilio - our keen thinkers from the earlier work "Home of the Brain". "Poli-tic-tic-tic", says Flusser's voice as a performer bows backwards and forwards and thus interprets a part of Flusser's words: "Youngsters at the terminals; they turn their backs to politics and turn to each other." This passage, from an interview

35 Body-Track is part of the eMuse-Systems, which were developed as a production system for "Murmuring Fields" (Strauss / Fleischmann 2007). 
with Vilém Flusser in 1990 on Austrian television, was sampled and built into the sound database. The dancer triggers syllables with her body and forms speech. She plays with the meaning of the concepts. Text is translated into a texture of sound and movement. Theatre Scientist Martina Leeker sees that "in such information-technological modifications of theatre and stage (...) the internalisation of thought and imagination are overridden inasmuch as the actors become bodily involved. The externalisation of thought and imagination through new media forms allows the mental 'Mixed Reality Room' to develop, and at the same to operate alongside our written cultural heritage." 36 In "Murmuring Fields" movement of the body moves sound and image. The art historian Oliver Grau judged that with "Murmuring Fields" a new type of space of mind had been created. ${ }^{37}$

\section{Media Art as Public Thinking Space}

The question of how digital information can translate not only the metaphoric virtual, but also the physically real, into accessible and understandable domains marked the passage to media architecture. By this we understand an architecture which connects people, space and data with one another. It creates an extended area of activity. Our first experiment interlinking data and virtual space resulted in "Home of the Brain", an immersive environment for a single person. Connected to this work we developed "Murmuring Fields", a sound environment for two performers on a public stage. With "EnergiePassagen"38 (2004) we explored data connected to public space with an audience passing by day and night. It was strolling through news: promenading through information space. This model of public space was possible for us, following the inclusion of text and natural language, after our research and development of linguistic tools. The project "Energie-Passagen" is about reading and describing the city. It schematises public and private interest in information. This public installation offers the possibility of association, filtering and choice of a flow of words, which allow participant-oriented opinion formation and therefore a public interactive thinking space (Denkraum). ${ }^{39}$

The starting point of "Energie-Passagen" is texts from mass-media daily news. An automatic computer technique analyses the daily newspaper and reduces it to the 500 most-used keywords. In this case it was the "Süddeutsche Zeitung", which was analysed over four weeks by a semantic tool. The filtered terms appeared as a large screen-projected "flow of infor-

36 Leeker 2000

37 Grau 2004

38 <http://energie-passagen.de>

39 The notion of the German Denkraum refers to Aby Warburg and Hanna A,rendt. 


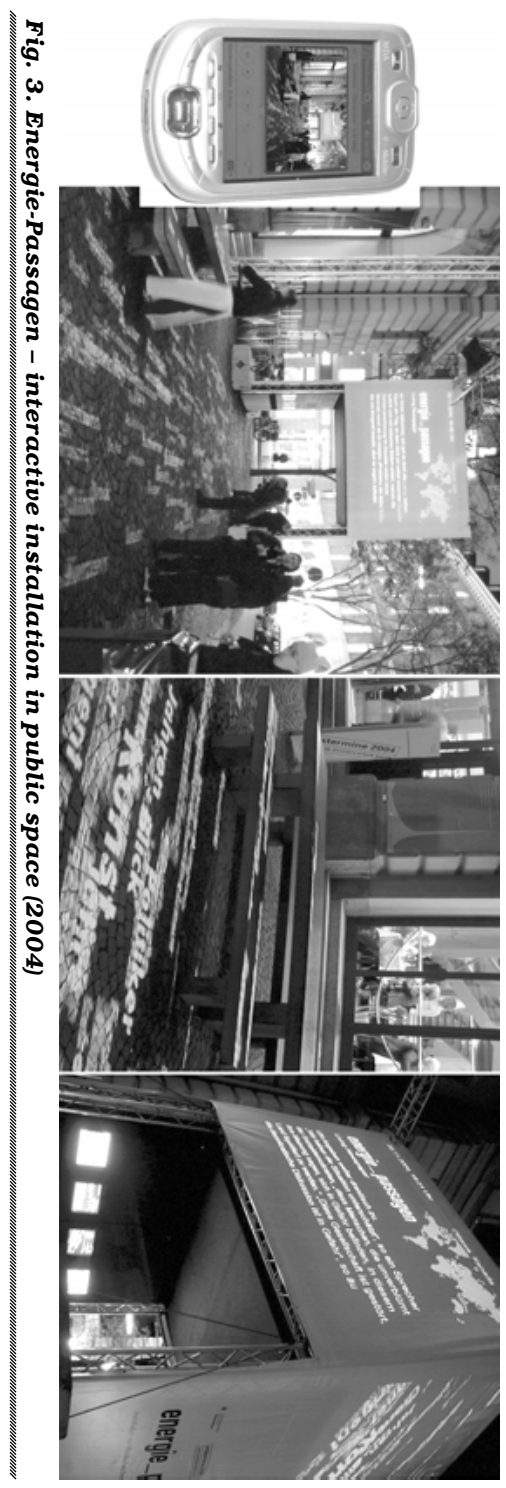

mation" in November 2004 in front of the "Literaturhaus" in Munich. Visitors could choose definitions onsite and "interpose" them into the flow. In this way text movements are set into motion, which allow connections between the definitions to emerge. The definition network creates new meanings, which differentiate themselves from the original linear texts. Computer voices react directly to the intervention of the visitor and accompany him or her as multi-voiced echo. In addition, a world map visualises the journey, which then takes the chosen definition through the geographic landscape of the news. The visible result of the visitors' preferred words is the "Living Newspaper". Its dynamically generated choice is projected within their original sentence onto the "information cube". The deconstruction of the newspaper, which results from the fragmentation of its original contents, leads to an unaccustomed reading and understanding. The artificial voices of the flow invite the discovery of new sense correlations. The spectators find themselves in an aura of speech and luminous symbols. It creates an atmosphere of liveness between audience and place that allows immersion (consumption) as well as reflexion (evaluation).

Words in motions set the visitors' own associations free. This unusual view of familiar daily news leads to surprising actions and reactions. Every day and evening of the four weeks, the on-site public discussed their own associations related to the daily news reports. An especially pleasing aspect was the discussions with older visitors, who felt themselves involved in the current developments and enjoyed 
being able to experience something other than established forms of art. With "Energie-Passagen" we schematise speech in public space. The work shows how meaning can emerge through deviation, and offers the on-site public a sensual, tangible space for action, which sets free new potential for communication. Communicative performance and performative presence emerge through interactivity of audience and virtual space. The greater part of the approximately 4000 on-site visitors to the installation were between 50 and 70 years old. During the one-month duration of the installation there were a further 3,000 internet visitors, some of whom also sent comments. ${ }^{40}$ More than four years later, in June 2008, there have been nearly 50,000 visitors who have seen the installation on the internet. ${ }^{41}$ The audience on site creates performative presence. The interactive spectator online creates a different form of performative presence with the web installation.

\section{Conclusion}

Film lives from its heroes, whilst the interactive players can themselves be actors and heroes - even in an art installation. Film needs an audience which gets inside a story and loses itself in it. This narrative art usually requires a plot, which includes a storyline and action. Normally, a plot is characterised by a beginning, middle and an end. This rule does not apply to interactive stories. Interactive media art assumes that observers are themselves active. Thus, the activity of participants can be interpreted as communicative performance. One's own participation is a precondition for the experience of story and plot.

The core of the interactive plot is not about the communication of one single reality, but lies in differing viewpoints and positions based on hypertext structures and generative processes. The interactive performance is more about the volatile association of thoughts than the linear narrative of a film. As an analogy, one can describe the process of the exposure to interactive installations as thought in action and action in thought. This in turn indicates making visible the activity of thought processes. Communicative performance and performative presence evoke the atmosphere of the artwork as a thinking space (Denkraum).

A shortened re-working from Medienkunst im Aufbruch - Geschichten erzählen von interaktiv bis reaktiv, ed. Klaus Rebensburg, Aachen: Shaker 2005, pp.65-84

40 Visitors' comments: see <http://www.energie-passagen.de/presse2.html> (last access: June 2008).

41 Energie-Passagen as a web installation: see <http://www.energie-passagen.de/ webinstallation> (last access: June 2008). 


\section{References}

Bates, John (1991): Virtual Reality, Art, and Entertainment. Online available: <http:// www.soe.ucsc.edu/classes/cmps 148/Spring08/BatesVRAE.pdf> (last access: June 2008).

Bleecker, Julian/Knowlton, Jeff (2006): »Locative Media: A Brief Bibliography And Taxonomy of GPS-Enabled Locative Media«. In : Leonardo 14(3). Online available: <http: / /leoalmanac.org/journal/Vol_14/lea_v14_n03-04/jbleecker.asp> (last access: Juli 2008)

Fischer-Lichte, Erika (2008): »Reality and Fiction in Contemporary Theatre«. Theatre Research International 33(1), 84-96.

Fleischmann, Monika/Strauss, Wolfgang (Art+Com) (1990-1992): »Home of the Brain«. Online available: <http://netzspannung.org/database/home-of-the-brain/ en> (last access: June 2008).

Fleischmann, Monika/Strauss, Wolfgang (1997-99): »Mixed Reality Performance Space«. Online available: <http://netzspannung.org/cat/servlet/CatServlet?cmd =netzkollektor\&subCommand=showEntry\&entryId=148563\&lang=en $>$ (last access: July 2008).

Gemeinböck, Petra (2004): »Negotiating the In-Between: Space, Body and the Condition of the Virtual«. Crossings: eJournal of Art and Technology 4(1). Online available: <http: / crossings.tcd.ie/issues/4.1/Gemeinboeck/> (last access: October 2007).

Gilliatt, Penelope (1970): »After Man«. Reprinted from The New Yorker. In: Jerome Agel (Ed.), The Making of Kubrick's 2001, New York: The New American Library.

Grau, Oliver (2003): Virtual Art. From Illusion to Immersion, Cambridge: MIT Press.

Grau, Oliver (2004): »Immersion und Interaktion. Vom Rundfresko zum interaktiven Bildraum«. In: MedienKunstNetz. Online available: <http://www.medienkunstnetz. $\mathrm{de} /$ themen/medienkunst_im_ueberblick/immersion/scroll> (last access: October 2007).

Leeker, Martina (1999): »Theater, Tanz, Performance and Informationstechnologie: Die Rolle des Schauspielers und des Publikums in Mixed Realities « (= lecture at the "Memoria Futura" Symposium of the GMD, 2000). Online available: <http://maus. gmd.de/imk_web-pre2000/docs/ww/mars/cat/memoria/leeker_dt.htm> (last access July 2008).

Packer, Randall/Jordan, Ken (2002): »Multimedia: From Wagner to Virtual Reality«. Online available: <http://www.artmuseum.net/w2vr/Book.html\#Book> (last access: October 2007).

Penny, Simon (1995): Critical Issues in Electronic, New York: State University of New York Press.

Strauss, Wolfgang et al. (1999): „Staging the space of mixed reality - reconsidering the concept of a multi-user environment«. In: Proceedings of the 4th Symposium on Virtual Reality Modeling Language, New York: ACM, 93-98.

\section{0}


Strauss, Wolfgang/Fleischmann, Monika (2007): »Imagine Space Fused with Data«. In: Cast01 Proc. 2001. Online available: <http://netzspannung.org/version 1/cast01/ proceedings/index.html> (last access: November 2007).

Sutherland, Ivan E. (1965): „The Ultimate Display«. In: Proceedings of the IFIP Congress 2, 506-508. 


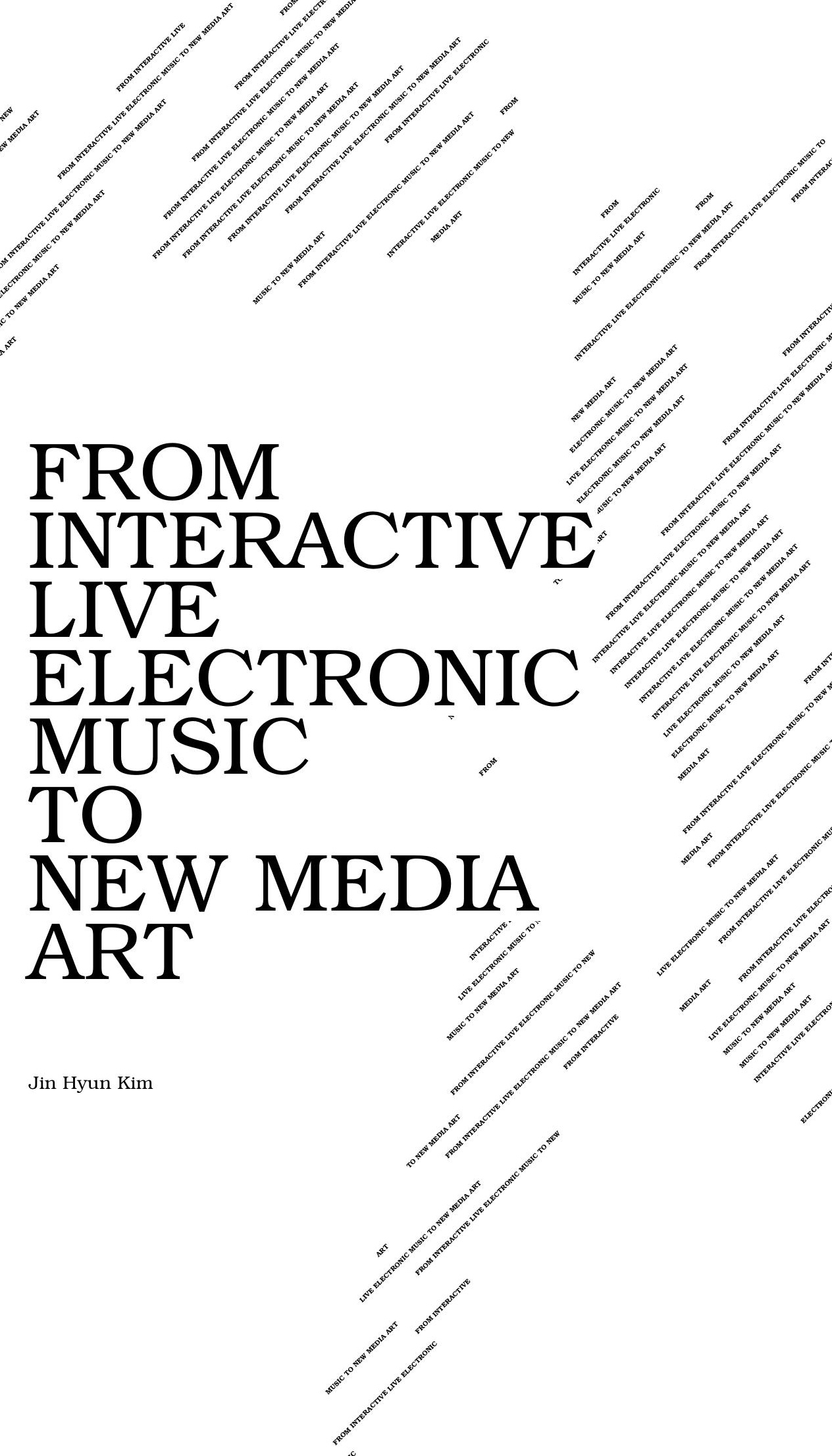




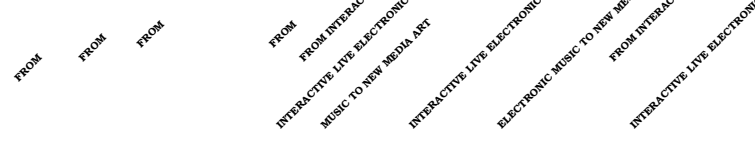

\section{Prologue}

Recent diverse artistic projects using interactive technologies are indeed complex, but many of them together form a new direction, which relocates and dissolves traditional boundaries between different categories of art. This article is particularly concerned with a shift related to the concept of music. In the culture preserving the heritage of Western art music, music often refers to works of art consisting of well-formed sound structures which are free of any purpose, i.e. autonomous, and can be understood by distant attentional listening. This understanding of music as an autonomous work of art was supported by autonomous aesthetics grounded in romanticism on the one hand, and by the philosophy of history oriented towards the idea of progress of Western history towards the point of modern times on the other. ${ }^{1}$

Even though metanarrations acting as a legitimation of Western art music began losing their validity at least due to the postmodern discourses on delegitimation and plurality, ${ }^{2}$ it is remarkable that discourses on legitimation of one category of music definitely decay in the digital era. It may be observed that there is an unexpected shift from a traditional concept of music, due to the essentially changed format of production and reception of "art" and of formation of artistic experience in New Media Art, even in the cases where sonic materials are primarily used and therefore auditory perception is strongly engaged.

\section{"Interactivity" as a key concept calling into question a traditional understanding of music in the Western music tradition}

"Interactivity" is a concept which came into focus in Western music tradition through a dadaistic trend, in which John Cage is a key character. ${ }^{3}$ This concept is closely related to questioning the traditional concept of music as an autonomous work of art which is considered as intentionally produced by a 'genius' on the one hand and as receptively perceived by the audience on the other. Cage aims at 'indeterminacy' of the relationship between composition and realisation using a graphical, meaning-free notational system with which musical parameters are not determined as absolute variables, but in a relative relation to each other. He carried out the performances of happenings and fluxus arts with Merce Cunningham, Robert Rauschenberg, and

1 Kim 2004, Chapter 3-4

$2 \quad$ Lyotard 1979; Kim 2004, Chapter 6

3 I refer to "Western music tradition", since the concept of interactivity should be discussed from a different point of view when taking into account other musical cultures.

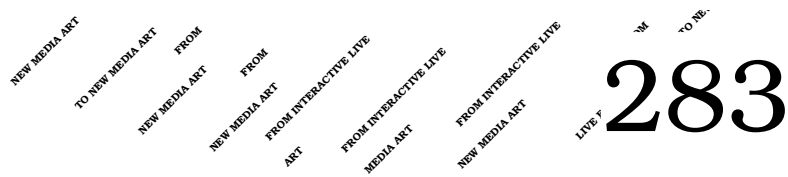


David Tudor, among others, at the Black Mountain College in North Carolina (U.S.A.) at the beginning of the 1950s, reformulating the concept of art and the relationship between artist, work of art, audience/public and environment: In these performances, creativity is not manifested in a durable work of art, but brings forth action. "Interactivity" becomes a core concept which makes a musical work emerge from the interplay between the composer, the performer, and the audience, from which an unpredictable effect arises during each processual performance. In this way, the artist becomes part of her or his work of art. ${ }^{4}$

Cage opens a new conception of music. Music becomes an aesthetic experience of the unpredictable, the source of which can be found elsewhere than in the intention of a musician being considered as the subject of music. Musical performance is not conceived of as a stage on which an intentional subject expresses her- or himself, but as an occasional situation in which sounds come into the focus of attention. Such desubjectivisation gives rise to the demise of the progress idea of musical structure underlying Western art music, which is oriented towards the modern philosophy of history, and furthermore to the abolition of the category of the 'closed' work of art into an open aesthetic process.

Cage's musical compositions include diverse experiments with musical materials, instruments, and performance constellations. However, it is remarkable that he is one of the pioneers in new directions of music composed electronically. ${ }^{5}$ In the 1930s and 1940s he used a film phonograph and electronic musical instruments (e.g. "Novachord", a polyphonic synthesiser manufactured by Hammond, and "Theremin", an antennae-based musical instrument played with free hand gestures) for The Future of Music: Credo (1937) and oscillators, turntables, and generators for his composition series Imaginary Landscape No. 1-4 (1939-1951). The use of sound materials created by electronic means and the exploration of different electrotechnical methods of sound collection, generation, and control are closely related to his search for a new concept of music.

In the 1950s and 1960s, after he introduced the principle of "indeterminacy", a live character was assigned to his compositions for sounds created electronically, taking the process of electronic sound generation out of the studio. A musical event of electronic sounds which is formed as composition results from a series of actions which can vary in each performance. A predetermined compositional idea is only concerned with the whole structure including duration, possible actions, sound materials prepared, and if

\section{$4 \quad$ Harris 1987}

5 Since the term "Electronic Music" is generally assigned to the musical genre in which a sine wave generator has been used for sound generation in Cologne since the 1950s, I avoid applying this term to early compositions of Cage.

\section{4}


necessary a kind of dramaturgy. The completion of this composition relies rather on (partially unpredictable) performative actions. Variations V (1965), which Cage realised with Merce Cunningham as an interactive composition for dancers, can be taken as an example. The performance stage for dancers equipped with antennae measuring the electromagnetic capacities produced by the various distances from the dancers to each antenna, and with photoelectrical cells measuring light conditions on the stage changed by the dancing movements - serves as a stage for actions which lead to musical interaction between pre-recorded sound materials on tape recorders and short wave radios resulting in a sound mix. With the vertical movements of their bodily parts, the dancers were capable of influencing the sound intensity; the horizontal direction of dancing movements led to varying proportions of sound distribution on the different amplifiers. In his Remarks 37 (1965), Cage makes it clear that the composition of Variations $V$ consists in the medial configuration, i.e. elements of sound system and control units. ${ }^{6}$

\section{The early stage of interactive live electronic music}

The experiments with live electronic music which Cage's new concept of music and musical composition underlies, however, seem to derive from the main trend of live electronic music, taking into account, in particular, the European scene of live electronic music. Even though an integration of live music performance into concerts of Electronic Music is also an essential aspect of the latter, the idea of interactivity which Cage explicitly deals with, questioning the concept of Western art music as autonomous work of art, is hardly found in the early stage of live electronic music rooted in European Musique Concrète and Electronic Music. ${ }^{7}$ This might consist in the fact that a number of the composers of Musique Concrète and Electronic Music tend to extend new musical materials and electrotechnical procedures to create an autonomous musical piece, following the tradition of Western art music.

In most performances of live electronic music, composers tried to experiment with the possibilities of integrating live performing musicians (singers and instrumentalists) into the performances of electronic music, which otherwise are purely based on the reproduction of a pre-composed piece via

$6 \quad$ Cage 1965

7 The French sound engineer and composer Pierre Schaeffer founded Musique Concrète, which is created with "concrete" sound objects by an electronic means. Everyday noises, speech sound, or acoustic sounds are recorded using a microphone on magnet tape and processed using montage, filtering, mix, and transposition etc. (Schaeffer 1966). The centre for Electronic Music in Cologne was engaged in techniques of sound generation in a purely electronical way. Sinus tones were generated by an generator, and then either overlapped or mixed with filtered noises and impulses and recorded on a tape (Manning 1985). 
loudspeakers. In this way, they aimed at the interplay between traditional (live) music performance and reproduction of electronic musical compositions. Live electronic music can in this context be seen rather as a complementary approach to traditional electronic music performances, giving them a live stage character and enabling the use of an ensemble of instrumental (or vocal) and electronic music. A relocation of the roles of composer, performer, and the audience or the concept of music as a work of art, however, does not seem to be a topic in the European trends of live electronic music.

Interactive live electronic music has been developed in the context of live electronic music as an approach to solving the lack of context-sensitive variations of usual live electronic music consisting of a live performance of instrumental or vocal music accompanied by pre-composed, fixed tape music. Interactive live electronic music tries to render electronic music parts capable of reacting to live performance situations and varying contextsensitively according to each performance situation. For this purpose, a computer-aided analysis of information coming from live performance - utilising interactive software - serves as a basis for an 'interactive' output of electronic sounds. "Interactivity" is here related to the capacity of the computer system to "change [its behaviour] in response to musical input", as the composer and researcher of computer music Robert Rowe defines "interactive music systems". ${ }^{8}$ This is similar to a technical concept of interactivity used in early information technological research on human-computer interaction (HCI). In those so-called interactive music systems, the tasks of the computer consist in an interpretation of raw data captured during a live performance and in their use for musical composition and for sound generation. In the early stage of live electronic music, the role of interactive music systems was computeraided automatic accompaniment of a live performance. The composer Joel Chadabe who introduced the term "interactive composing" in 1983 gives an overview of an (early) interactive music system (Fig. 1).

In most performances of early interactive live electronic music, musical information - e.g. pitch, loudness, dynamics etc. - served as the input data of interactive music systems. The so-called score following techniques were developed for this purpose: ${ }^{9} \mathrm{~A}$ musical score for the live performance is put into a computer system in a certain form. The live performance is captured via a microphone or a MIDI interface and analysed by the computer system in real-time. The analysed sound events are compared to the score stored in the computer. If there is a match, the computer accompanies the live performance, generating sound events algorithmically - based on the score storage.

8 Rowe 1993, p. 1; This book entitled "Interactive Music Systems" is the first book dealing with those systems and Interactive Music systematically.

9 Vercoe 1984; Dannenberg 1984

\section{6}




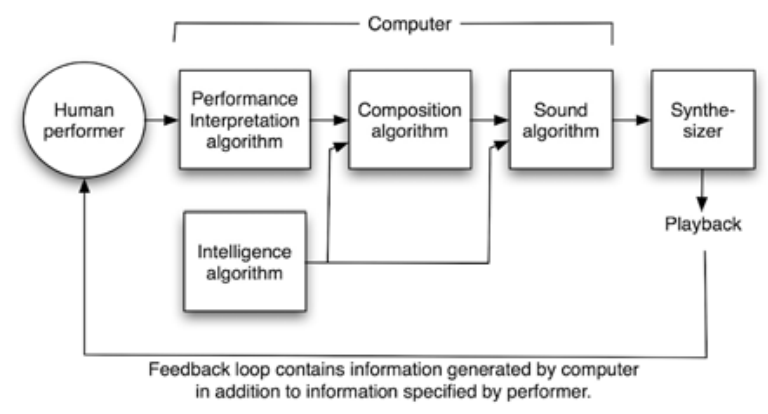

Fig. 1. The general principle of procedure of an interactive music system (Chadabe 1983)

Most score following techniques, however, are based on the principle of a knowledge-based system developed by a traditional approach of artificial intelligence. A musical score, which is put into a computer system, acts as a kind of represented knowledge. A score-following technique, which allows the computer system to monitor input events coming from live performances of an instrumentalist and to compare these with the knowledge - the score - of the computer system so as to process computer-generated sound parts, has a hierarchical structure of interaction processes - from the sensing up to the processing and down to the response stage. ${ }^{10}$ A knowledge-based process of interpretation of information coming from the sensing stage takes place in the processing stage, which is separated from the sensing and response stage. In other words, an exchange between internal and external processes does not take place during the processing stage. Output events of machines as a response to input events are determined in this isolated stage and realised by top-down organisation. Hence, knowledge-based interactive music systems are conceived of as decoupled from the environment and therefore as not truly interactive. What is realised in the early form of interactive live electronic music is a more flexible accompaniment of electronic music generated by algorithms to a live instrumental or vocal performance. In most cases, the traditional concept of music as "work of art" still remains.

\section{Bodily-based interaction with sound events}

Contrary to score following techniques, which are almost exclusively used for interactive live electronic music originating from the tradition of Western art music, further techniques of motion tracking are applied in broad artis-

10 Rowe 1993 
tic contexts. Since the 1980 s, in which technological possibilities to capture bodily actions and to use them as input data for the computer system were standardised, many artists have increasingly experimented with a variety of interactive art based on bodily actions of an observer/performer, whether interactive sculpture, installation, or performance.

In interactive live electronic music, motion tracking techniques, which became the focus of attention in the 1980s, have met with large interest since the 1990s, so as to lead to diverse experiments from both artistic and information technological perspectives. Motion tracking techniques are combined with so-called gesture mapping, which means an effective computable mapping from bodily gestural parameters (e.g. position of a body part or intensity of finger pressure) into parameters for sound synthesis (e.g. frequency or amplitude of acoustic wave form). Hence the development of different strategies of gesture mapping has become a hot issue of information technological research on interactive live electronic music. Most of gesture mapping, however, consists of ad-hoc solutions, not based on a general rule. Gesture mapping, however, seems to offer diverse possibilities of designing the interactive relationship between bodily actions and sound events.

Musical interaction which is not based on score-following techniques, but on strategies of gesture mapping, does not only behave as an "ensemble" (live performer and computer accompaniment). Some modes of musical interac-

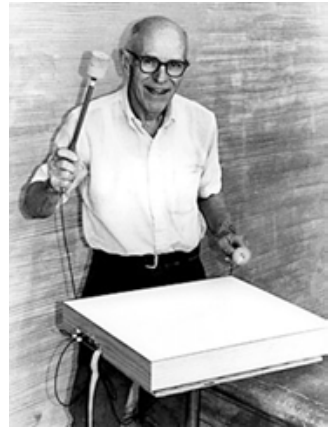

Fig. 2. Max Mathews with the Radio Baton/ Drum in 1992. Photo by Patte Wood (Chadabe 1997, p. 231) tion can also be described with further metaphors inspired by traditional music practices such as playing an instrument or conducting. A multiplicity of musical interfaces simulating, extending or re-configuring traditional musical instruments have been used in interactive live performances in which a performer has the role of a player of this new "instrument" and the computer acts as a musical instrument. ${ }^{11}$ The definition of a rule of gesture mapping is a main task of composition, which however is often not decoupled from performance. Therefore most composers act at the same time as a performer "playing" her or his musical interface designed especially for her or his compositions, which can be realised and completed during the process of performance.

Some interfaces allow the performer to act as a conductor shaping a musical composition expressively. For example, the Radio Baton/Drum developed by Max Mathews (Fig. 2) renders the performer

11 Editorial footnote: see for a few examples Jäger/Kim and Goto for an example of his violin interface SuperPolm in this book.

\section{8}


capable of controlling musical expressiveness during the performance, moving two batons equipped with different radio emitters over a square surface equipped with receivers. Each baton provides information about its horizontal $(\mathrm{x})$ and vertical (y) position and its height ( $\mathrm{z}$ ). In this way, a three-dimensional movement of each baton can be followed and mapped into parameters for digital sound manipulation. ${ }^{12}$

Such modes of musical interaction inspired by traditional music practices give rise to a rethinking of principles of bodily-based musical interaction, which is basic for each music performance. Especially the coordination of auditory and tactile sensations has become the focus of newer research on the design of musical interfaces based on approaches of physical, haptic, and tangible computing. ${ }^{13}$ A number of so-called haptic musical interfaces are capable of offering haptic feedback so that a user/performer can touch, press, or pull a physical material to enter into musical interaction mediated by algorithmic computation. A group of tangible interfaces can be grasped, squeezed, or moved from one place to the other so that a user/performer can use physical actions with the help of physical objects directly situated in a real environment to control and represent digital information. ${ }^{14}$ Furthermore, force feedback or vibrotactile feedback have been additionally simulated in some musical interfaces in order to improve musical interaction with new interfaces. Usually a user/performer dealing with haptic or tangible musical interfaces is requested to concentrate on physical actions which are rarely guided by visual representation. Hence she or he can develop the skill of coordination between tactile and auditory feedback the computer system offers, which is similar to the experience underlying the playing of traditional musical instruments. In this way, music can be dynamically composed, "feeling" it at the same time during the bodily-based interactive performance guided by a loop of double feedbacks.

Some strategies of gesture mapping enable a kind of dance-music interaction. The whole bodily movement of a performer can be tracked to trigger and control sound events. Dancing here no longer means an adjustment to pre-composed music, but a process of composing and modifying a musical structure which adjusts to dancing movements. A choreography of dance

\section{Boulanger/Mathews 1997}

13 Igoe/O'Sullivan 2004, Brewster/Murray-Smith 2001; McGookin/Brewster 2008; for information about current research on tangible computing see Proceedings of the 1st International Conference on Tangible and Embedded Interaction 2007, online available: <http: / / portal.acm.org/toc.cfm?id=1226969\&type=proceeding>; Proceedings of the 2nd International Conference on Tangible and Embedded Interaction 2008, online available: <http: / / portal.acm.org / toc.cfm?id=1347390\&idx=SERIES1 1433\&type=proceeding\&col $\mathrm{l}=\mathrm{ACM} \& \mathrm{dl}=\mathrm{ACM} \&$ part=series $\&$ WantType $=$ Proceedings $\&$ title $=\mathrm{TEI} \& \mathrm{CFID}=17833818 \& \mathrm{CFT}$ OKEN=87925403> (last access: March 2008).

14 Editorial footnote: see for some examples of tangible musical interfaces Weinberg in this book. 
serves here not as an interpretation of musical composition, but rather as a main part of musical composition which can only be realised by dancing performance. A complete composition which can be reproduced independent of each performance does not exist. Experimental computer-aided dance-music interaction is a new field which dissolves traditional categories of dance and music. Besides camera tracking techniques, some wearable interfaces such as the DIEM digital dance system by the researchers at the Danish Institute of Electroacoustic Music, the MIDI dancer by the artists group Troika Ranch and the SSPeaPer (Sensor/Speaker Performance Interface) by the composer and researcher Curtis Bahn have been developed especially for the purpose of dance-music interaction.

The principle of dance-music interaction also underlies many interactive sound installations which are not from a stage-oriented performance genre, but originate from the category of fine arts. Interaction often takes place in this context involuntarily, such as by entering into an installation room and triggering a sound generator. Further modes of interaction with sound events, however, can be actively explored. This act of interactive exploration may be compared to dancing. For instance, the Very Nervous System developed by the Canadian media artist David Rokeby is used both for dance-music interactions and for interactive sound installations. The Very Nervous System, which is based on a camera-tracking technique, is a sonically oriented system, since a virtual environment designed by Rokeby does not provide a visual representation, but consists solely of sound events. Therefore an observer cannot remain passive in order to enter into a computer-generated world, but attains an artistic experience only through an active improvisation. Rokeby intended to develop an improvisation system with which an observer/performer can explore an interactive relationship between her or his dancing actions and the sonifying installation environment. ${ }^{15}$ Rokeby's Very Nervous System views each movement not as an individual static image, but as a movement flow in the context of linear movement sequence. The temporal aspect comes in via the computation of movement analysis. The movement of the observer/ performer is interpreted in a horizontal linear flow, so that a certain movement can be transformed into different sound events according to the whole movement context. The interaction that the Very Nervous System provides is not based on the metaphor of dialogue which underlies the most traditional approaches of HCI. According to Rokeby, a dialogue implies a separation of functions of perception and reaction. ${ }^{16}$ His system, however, organises per-

\section{0}



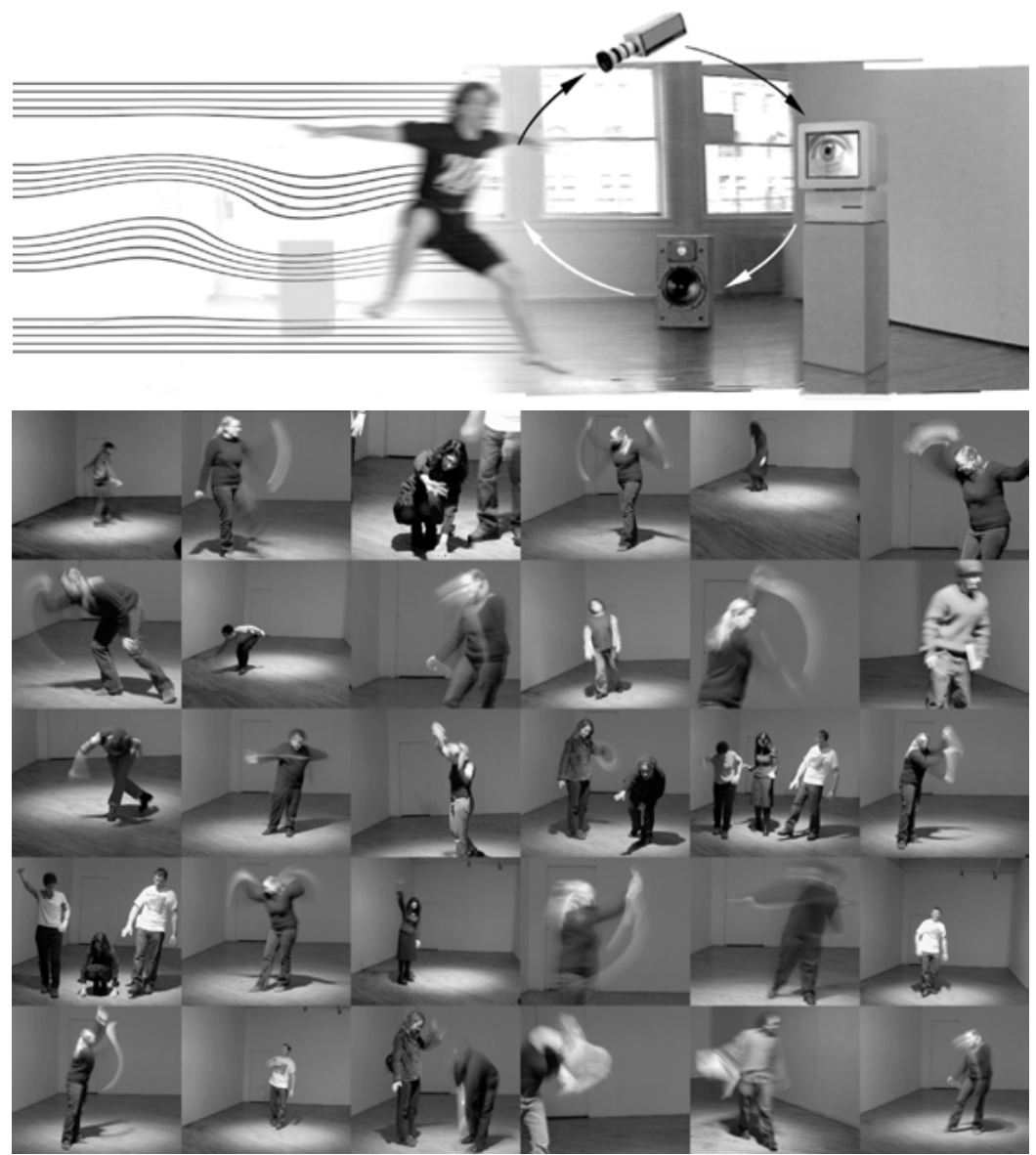

Fig. 3. Very Nervous System. Courtesy of David Rokeby

ception and expression at the same time, so that the observer and the computer system form a loop of feedback which is very close and complex. ${ }^{17}$

\section{Interactive emergence}

The concept of interactivity has been a topic which in turn needs rethinking due to the recent approaches of information technological research, artificial intelligence, and cognitive science, which place emphasis on new para-

17 Rokeby 1990 
digms questioning traditional concepts and their relations (e.g. perception, cognition, action). New technological methods which have been developed in the course of paradigm shift flow into New Media Art, which acts partially as an experimental environment for theoretical questions.

Aritificial Life (A-Life) Art, for instance, is a typical field which was instigated utilising a newer approach of artificial intelligence, A-Life procedures. ${ }^{18}$ A-Life uses the concepts of information processing and computational modeling to understand life in general. ${ }^{19}$ A-Life research aims at the definition of simple rules from which a complex behaviour emerges ${ }^{20}$ - contrary to traditional approaches of artificial intelligence, which give a machine a task to be solved, writing a program accordingly so that the machine can execute this task. Hence A-Life procedures focus on simple processes interacting among each other and in this way generating a high-order system behaviour. ${ }^{21}$ A-Life Art is based on a procedure to generate living behaviour of artificial agents, which is characterised as a bottom-up approach. An A-Life approach of computational modeling has recourse to the biological nature of creatures, so that properties such as self-organisation, emergence, reproduction, and adaption are assigned to a machine. Artificial Life (A-Life) Art accordingly experiments with different visual, sonic, or physical agents (e.g. robots) which show an emergent behaviour.

In communities of computer music research, a discourse on live algorithms for music (= LAM) has very recently been instigated, which is also the title of a series of conferences taking place since 2004. The main interest is directed towards autonomous interactive algorithms that are characterised by "adaptation and creative contributions of algorithms to the musical dimensions of sound, time and structure."22 It is concerned with interactive aspects of algorithms inspired by swarm intelligence, evolutionary computation, artificial life and complex dynamics. Live algorithms are intended to avoid "systems pre-loaded with syntax derived from music theory" and "rule-based approaches that relate input to output in a simple way." 23 Some composers and media artists who are engaged in interactive composition and improvisation up to now have experimented with live algorithms approaches in which interactivity is characterised by emergence.

The interactive sound installation Natural Selection (2005) by Tom Davis and Pedro Rebelo can be taken as an example of LAM projects. Davis and Rebelo use ten mechanical "sound objects", each of which consists of a reso-

18 Bird/Webster 2001; Sommerer 2001; Wilson 2003; Whitelaw 2004

19 Boden 1996, p. 1

20 Boden 1996, pp. 3-4; Braitenberg 1984

21 Boden 1996, p. 4

22 See <www. livealgorithms.org> (last access: March 2008).

23 <www. livealgorithms.org>

\section{2}


nator driven by a motor. Interaction takes place both among these sound objects and between them and the observer. Natural Selection is based on an algorithm which is inspired by frogs' behaviour, especially a female frog's choice of her mating partner out of the calling chorus of male frogs. ${ }^{24}$ Davis and Rebelo summarise relevant properties of frogs' mating calls from current research results and model them in sonic behaviour of the installation Natural Selection: The dominant call frequency is related to the size of the frog, the pulse

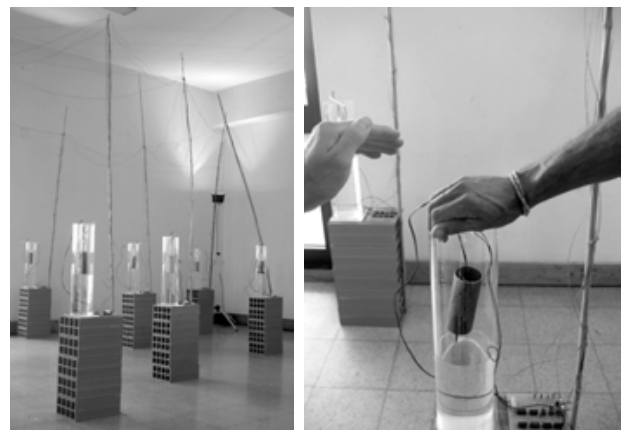

Fig. 4. The installation Natural Selection (2005) at the Música Viva-Festival in Portugal. Courtesy of Tom Davis rate to the temperature of the environment, and call rate and duration to the preference of each individual creature. ${ }^{25}$ Natural Selection uses a simple model of interactions between male frogs in a chorus, which are symbolised as sound objects, while the observer has a role of the female frog (Fig. 4).

The researchers from the University of Tokyo Jean-Julien Aucouturier, Yuta Ogai, and Takashi Ikegami have very recently experimented with a technique to make a robot dance to music autonomously and synchronously. They avoided a pre-programming of dance patterns. Instead, they built basic dynamics into the robot which render it capable of developing emergent behaviour. The dance movements of the robot were controlled by motor commands generated by using an artificial neural network (ANN), a network of artificial spiking neurons, each controlled by a biologically-inspired model (FitzHughNagumo (FHN)). ${ }^{26}$ A sequence of pulses detected from the beats of the music was processed by this ANN, and the output of the FHN network was mapped into the sequence of pulses being used for the robot dance corresponding to the beats of the music. Although this project has not been applied in an artistic context yet, the increasing number of robotic art and musical robotics projects indicates the current directions of using robots as agents of A-Life, contrary to traditional approaches of robotics based on top-down rules. ${ }^{27}$

24 Davis / Rebelo 2005

25 Davis/Rebelo 2005, section 2

26 Aucouturier/Ogai/Ikegami 2007, available online: <http://www.jj-aucouturier. info/papers / ICONIP-2007.pdf>

27 For detailed discussion on musical robotics projects see Seifert/Kim 2007, 2008. 
Christoph Lischka's project par_cho $\mid r$ (2001-2004), which exists in different implementations such as par_cho $\mid r$ : mono, par_cho $\mid r$ :fugue (as performances) and par_cho $\mid r$ : trans (as an installation), deals with sound-generat-

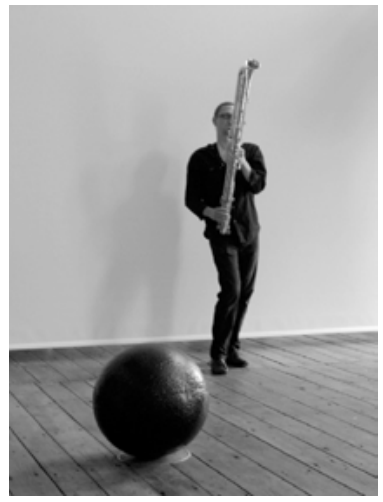

Fig. 5. par_cho|r : fugue. Performance at the International Symposium on Music, Art, and Robotics (SMARt) in Bremen (14 June 2006). Courtesy of Christoph Lischka ing algorithms embodied in the form of a ball robot. In performance projects, a human bass clarinettist interacts live via a 'sound language' with a ball robot which 'listens to' and 'analyses' music played on a contrabassoon and acquires in this way some kind of 'hearing' knowledge. ${ }^{28}$ Accordingly this ball robot moves within a certain defined space and plays a contra part. What is observed is an improvisation of two "musicians", which becomes evident in the emergent musical structure. ${ }^{29}$ Lischka describes the project as following: The project deals with "artificial ecologies, i.e. artificially generated 'creatures' with their ever particular environments." "An artificial lived-in world of 'ball creature' is created through simulation and reconstruction of this organism by sonic and ultrasonic sensor and actuator technologies. This 'ball creature' develops in each individual and collective characteristic (»Swarm«)

sonic, visual and choreographic interaction patterns which become in turn an object of artistic experience and performance."30

Such algorithms which allow a machine or/and a unity of human and machine to display emergent behaviour become an essential means of creating works of New Media Art. An experience with evolving sonic behaviour related to her or his behaviour makes an observer act as an actant ${ }^{31}$ who, however, does not always possess agency, but is affected at the same time as a patient. A true interaction is based on oscillation between agency and patienthood among the actants participating in this interaction, in a continuous circle of affecting and being affected, in short: reciprocal turn-taking. This concept of interactivity which allows an observer to shape a dynamic process of artistic creation leads to a calling into question of the connotation of "music", which is not limited to a concert hall for Western art music

28 <http: / / www.zeitmedien.de/AAS.html> (last access: March 2008)

29 Kim 2007

30 <http://www.zeitmedien.de/AAS.html> (translated quoting)

31 The term "actant" is used by Bruno Latour in the context of the actor-network theory to refer to entities which carry out an action. This term differs from that of "actor", which refers exclusively to an intentionally acting human subject (Latour 1996).

\section{4}


or opera house culture demanding only one musical behaviour: a passive distant behaviour of music listening. "Interactivity" as a main subject in New Media Art which is of great interest in our digital era makes clear the necessity to delegitimate the hegemony of Western art music and at the same time to rethink the nature of music, which may be conceived of as interactive from the outset. 


\section{References}

Aucouturier, Jean-Julien / Ogai, Yuta/Ikegami, Takashi (2007): »Making a Robot Dance to Music Using Chaotic Itinerancy in a Network of FitzHugh-Nagumo Neurons«. In: Proceedings of the 14th International Conference on Neural Information Processing (ICONIP 2007). Online available: <http://www.jj-aucouturier.info/papers/ ICONIP-2007.pdf> (last access: March 2008).

Bird, Jon/Webster, Andy (2001): »The Blurring of Art and Alife«. In: Proceedings of Second Iteration $\ll, 38-46$.

Boden, Margaret A. (Ed.) (1996): The Philosophy of Artificial Life, Oxford: Oxford University Press.

Boulanger, Richard/Max Mathews (1997): »The 1997 Mathews Radio-Baton and Improvisation Modes«. In: Proceedings of the 1997 International Computer Music Conference, San Francisco: International Computer Music Association, 395-398.

Braitenberg, Valentino (1984): Vehicles: Essays in Synthetic Psychology, Cambridge, MA: MIT Press.

Brewster, Stephen/Murray-Smith, Roderick (Eds.) (2001): Haptic Human-Computer Interaction, Berlin: Springer.

Cage, John (1965): Variations V. Thirty-Seven Remarks re an Audio-Visual Performance [score with remarks], New York: Henmar Press.

Chadabe, Joel (1983): »Interactive Composing: An Overview«. Computer Music Journal $8(1), 22-27$.

Chadabe, Joel (1997): Electric Sound. The Past and Promise of Electronic Music, New Jersey: Prentice-Hall.

Dannenberg, Roger (1984): »An On-Line Algorithm for Real-Time Accompaniment«. In: Proceedings of the 1984 International Computer Music Conference, San Francisco: International Computer Music Association, 193-198.

Davis, Tom/Rebelo, Pedro (2005): »Hearing Emergence: Towards Sound-Based SelfOrganisation«. In: Proceedings of the 2005 International Computer Music Conference. Online available: <http://www.sarc.qub.ac.uk/ tdavis/Hearing\%20Emergence. pdf> (last access: March 2008).

Harris, Mary Emma (1987): The Arts at Black Mountain College, Cambridge, MA: MIT Press.

Igoe, Tom/Dan O'Sullivan (2004): Physical Computing: Sensing and Controlling the Physical World with Computers, Boston MA: Course Technology PTR.

Kim, Jin Hyun (2004): Musikwissenschaft in der Postmoderne. Zur Legitimationsproblematik von Musikwissenschaft, Osnabrück: epOs.

Latour, Bruno (1996): »On Actor-Network Theory. A Few Classifications«. Soziale Welt 47(4), 369-381.

Lyotard, Jean-François (1979): La condition postmoderne: Rapport sur le savoir, Paris: Minuit.

\section{6}


McGookin, David / Brewster, Stephen (Ed.) (2008): Haptic and Audio Interaction Design: First International Workshop, HAID 2006, Glasgow, UK, August 31 - September 1, 2006, Proceedings (Lecture Notes in Computer Science), Berlin: Springer.

Manning, Peter (1985): Electronic and Computer Music, Oxford: Oxford University Press.

Rokeby, David (1990): »The Harmonics of Interaction«. Musicworks 46: Sound and Movement, Spring. Online available: <http://homepage.mac.com/davidrokeby/ harm.html> (last access: March 2008).

Rowe, Robert (1993): Interactive Music Systems: Machine Listening and Composing, Cambridge, MA: MIT Press.

Schaeffer, Pierre (1966): Traité des Objects Musicaux: Essai Interdisciplines, Paris: Seuil.

Seifert, Uwe/Kim, Jin Hyun (2007): »Entelechy and Embodiment in (Artistic) HumanComputer Interaction«. In: Julie A. Jacko (Ed.), Human-Computer Interaction, Part I, HCII 2007, LNCS 4550, Berlin/Heidelberg: Springer, 929-938.

Seifert, Uwe/Kim, Jin Hyun (2008): »Towards a Conceptual Framework and an Empirical Methodology in Research on Artistic Human-Computer and HumanRobot Interaction«. In: Aleksander Lazinica (Ed.), Advances in Human-Computer Interaction, Vienna: I-Tech Education and Publishing 2008, 177-194.

Sommerer, Christa (2001): »Special Section Introduction - A-Life in Art, Design, Edutainment, Games and Research«. Leonardo 34(4), 297-298.

Vercoe, Barry (1984): »The Synthetic Performer in the Context of Live Performance «. In: Proceedings of the 1984 International Computer Music Conference, San Francisco: International Computer Music Association, 199-200.

Whilelaw, Mitchell (2004): Metacreation. Art and Artificial Life, Cambridge MA: MIT Press.

Wilson, Stephen (2003): Information Arts: Intersections of Art, Science, and Technology, Cambridge MA: MIT Press. 
8

EXTENDING

THE

MUSICAL

EXPERIENCE

FROM

THE

PHYSICAL

TO

THE

DIGITAL

AND

$\mathrm{BACK}$ 


\section{Introduction}

It is widely perceived that the computer has enriched and advanced the art form of music. Digital technology brought new palettes of sounds, composition techniques, and production methods; innovations in digital compression and distribution changed music consumption and listening practices; for performers, novel musical instruments and controllers have been developed based on a variety of sensing, interaction, and mapping approaches. But after more than two decades of research in computer music, a fundamental question must be asked - has digital technology truly innovated and enriched the expressive, emotional, and creative core of the musical experience? It is not clear that the answer to this question is as positive as we music technologists would like to think.

During the last ten years, inspired and motivated by the prospect of innovating the core of the musical experience, I have explored a number of research directions in which meaningful use of digital technology bears the promise of revolutionising the medium. The research directions identified - gestural expression, collaborative networks, and constructionist learning - can lead to musical experiences that cannot be facilitated by traditional means. The first direction builds on the notion that through novel sensing and mapping techniques, new expressive musical gestures can be discovered that are not supported by current acoustic instruments. Such gestures, unconstrained by the physical limitation of acoustic sound production, can provide infinite possibilities for expressive and creative musical experiences for novice as well as trained musicians. The second research direction utilises the digital network in an effort to create new collaborative experiences, allowing players to take an active role in determining and influencing not only their own musical output but also that of their co-performers. By using the network to interdependently share and control musical materials in a group, musicians can combine their musical ideas into a constantly evolving collaborative musical activity that is novel and inspiring. The third research direction utilises constructionist learning, which bears the promise of revolutionising music education by providing hands-on access to programmable music making. Through interaction with physical computational objects, learners can construct personally meaningful musical artifacts that enhance and deepen their learning.

While facilitating novel musical experiences that cannot be achieved by traditional means, the digital nature of these research directions often leads to flat and inanimate speaker-generated sound, hampering the physical richness and visual expression of acoustic music. In my most recent work, therefore, I attempt to combine the benefits of digital computation and acoustic richness, by exploring the concept of "robotic musicianship". I define this concept as a combination of musical, perceptual, and social skills with the

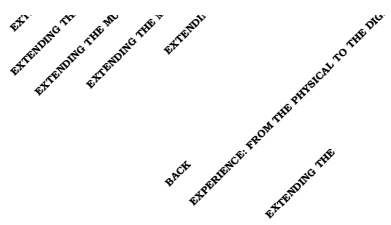


capacity to produce rich acoustic responses in a physical and visual manner. The robotic musicianship project aims to combine human creativity, emotion, and aesthetic judgment with computational capabilities, allowing human and robotic players to cooperate and build off one another's ideas. A perceptual and improvisatory robot can best facilitate such interactions by bringing the computer into the physical world both acoustically and visually.

In this paper I will describe my projects portraying a musical journey that was initiated by my interest in extending acoustic music with digital technology and reached its most recent period by investigating the enhancement of digital music through physical-acoustical means. Each station in this journey presents a different set of novel expressive and creative possibilities along with a set of limitations and constraints imposed by technology.

\section{Related Work, Goals, and Challenges}

The field of New Interfaces for Musical Expression ${ }^{1}$ has received significant interest in recent years as researchers and musicians explore new sensing techniques, design approaches, mapping schemes, and sound generation methods to enhance and enrich musical expression. Research in this area can be categorised into two main areas - Imitated and Augmented Instruments, and Alternate Controllers. Building on the vast repertoire of familiar musical gestures, researchers have created imitated and augmented versions of traditional instruments such as percussions, strings and woodwinds, among others. Alternative ways to play music have also been explored by using various sensing and mapping techniques such as in non-contact instruments wearable music and alternate tangible controller. Most of these instruments, however, have been created for particular compositions (usually by the inventor) and have been effective only within specific aesthetics boundaries. Only few controllers have shown durability and adaptability to multiple compositions in a variety of musical styles. Inspired by the tradition of great versatile acoustic instrument such as the piano, one of the main goals of my work was to develop controllers that are durable, versatile, and adaptable to multiple compositions, styles, and playing techniques.

The second area of related work is in the field of Interconnected Musical Networks (IMNs) - live performance systems that allow players to influence, share, and shape each others' music in real-time. Such systems, whether they operate in one physical space or over a wide-area network, provide an interdependent framework that can lead to rich social and musical experiences that are not supported by traditional group play. The development of

$1 \quad<$ http: / / www.nime.org >

\section{0}


IMNs since the 1950s has been connected to the development of technological innovations - from John Cage's early experimentations with interconnected transistor radios through the use of networked PCs by groups like the League of Automatic Music Composers and the Hub, to the current proliferation in collaborative Internet music. These experiments, however, usually require advanced musical skills and understanding by players and audiences, and often lead to inaccessible "high art" musical outcome. More recent collaborative musical installations for novices on the other hand, tend to simplify the musical experience for novices and are not geared to interdependently connect between novices and professionals. To address this gap, my work attempts to explore novel interdependent musical interactions that would provide both novices and experts with rich and inspiring, yet intuitive and easy to follow, collaborative musical experiences.

The educational goal of my research is informed by related work in the field of constructionist learning. The constructionist approach emphasises the unique ability of digital technology to provide personal and configurable learning experiences to a wide variety of learners. The approach was conceived by Seymour Papert, who demonstrated how learning is most effective when students construct personally meaningful technological artifacts. Other researchers have elaborated on Papert's ideas, showing how interaction with digital physical objects enhances children's and adults' learning. In music, however, little has been done to develop constructionist systems that attempt to connect between figural expressive musical experiences and formal aspects of theory and technique. In conventional music education systems, when music students are introduced to formal theory, certain important expressive aspects that came naturally in the early figural mode are temporarily hidden when learners try to superimpose analytical knowledge upon felt intuitions. My work attempts to utilise constructionist-learning methods to bridge the gap between the figural and formal learning modes through hands-on interaction with programmable musical controllers.

And lastly, I introduce the concept of robotic musicianship, taking up Rowe's concept of machine musicianship. In this research area, scholars develop interactive systems that analyse, perform, and compose music with computers based on theoretical foundations in fields such as music theory, computer music, music cognition, and artificial intelligence. Several effective approaches for the design of such interactive musical systems have been explored over the years by researchers and musicians such as Dannenberg ${ }^{2}$, Cope $^{3}$, Lewis ${ }^{4}$, Pachet ${ }^{5}$, and others. Such digital interactive systems, however,

2 Dannenberg 1984

3 Cope 1996

4 Lewis 2000

5 Pachet 2002 
are limited by the inanimate and flat nature of their digital musical reproduction. Current research directions in musical robotics, on the other hand, focus mostly on sound production and rarely address social aspects such as listening, analysis, group improvisation, or collaboration. Both "robotic instruments" 6 - mechanically automated devices that can be played by live musicians or triggered by pre-recorded sequences - and "anthropomorphic robots"7 - hominoid robots that attempt to imitate the action of human musicians - function mostly as mechanical apparatuses that follow deterministic rules. The motivation for establishing the field of robotic musicianship is to develop robots that can produce rich acoustic sound and visual cues, while utilising computational power and techniques of machine musicianship that are not possible with traditional acoustic instruments.

\section{The Projects}

\subsection{The Musical Playpen (1997-1998)}

The Musical Playpen was the framework for my preliminary experimentation with gestural musical interaction in a constructionist-learning environment. The instrument was designed for toddlers and infants in an effort to explore whether very young children can participate in a meaningful, active

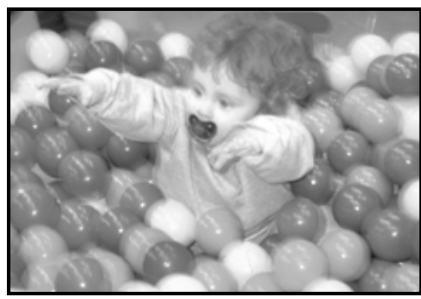

Fig. 1. A child playing in the Musical Playpen musical experience. The environment allows young children to control two highlevel musical aspects - contour and rhythmic stability - in an environment which is both familiar and fun: a $1.5-\mathrm{x}-1.5-\mathrm{m}$ playpen filled with 400 colourful plastic balls (Fig. 1). The playpen was designed to generate musical responses in correlation to children's activity. Players' movements around the playpen propagated from ball to ball and triggered four piezo-electric

sensors that were hidden inside four balls, one in each corner of the playpen. The balls' ability to transmit hits to neighboring balls, combined with the sensors' high sensitivity allowed for almost any delicate movement around the playpen to be captured by at least one sensor. The analog signal was then digitised and sent to a Macintosh computer running Max/MSP where it was mapped to musical output played from speakers below the playpen. Two opposite corners were mapped to control the melodic contour of an Indian raga,

6 For example, see Dannenberg et al. 2005, Jordà 2002, Singer et al. 2004.

7 For example, see Takanishi et al. 1998, Toyota 2004.

\section{2}


so that the more energetic the players' movements in these corners were, the higher the played Indian raga pitches became. Children could therefore create melodic phrases and manipulate their curves by changing the intensity of their body movements in these corners. Player's physical activity in the other two corners were mapped to an algorithm that controlled the tempo, rhythmic variation, and timbre of percussive sequences in an effort to provide access to controlling rhythmic stability. The more energetic the players were near these corners, the more versatile and uneven the rhythmic values became. The tempo curve also fluctuated more sharply, as did the rate of timbral change.

A number of observation sessions were conducted with the playpen at MIT and at the Boston Children's Museum from 1998 to 1999. These sessions have shown a wide range of responses to the environment and the high-level musical control that it offered. For example, a 1-year-old infant started her session by triggering a sequence of notes as she was placed near one of the melodic curve corners. The infant looked in the direction of the sound source and tried to move her hand towards that corner, seemingly trying to repeat the music she heard. When she succeeded and another melodic phrase was played, she smiled, took one ball and tried to shake it, obviously without audible results. Frustrated, she then threw the ball towards a rhythmic corner, generating a short percussive sequence. She approached this corner while moving her torso back and forth, laughing when discovering that her movements controlled the music. After a short break the infant started to move her body again back and forth, gradually accelerating her movements, generating less and less stable percussive sequences. Only after repeating this behaviour in another corner did the infant seem to be ready to use more expressive, less restricted gestures all over the playpen.

These responses can indicate that with the right instruments and controls, young children can have access to spontaneous, expressive musicmaking as well as to more serious and thoughtful musical explorations. These findings encouraged me to develop a new set of instruments, which I entitled "The Squeezables", in an effort to continue and develop models for high-level musical control, and to explore novel methods for networked group collaboration with older players, who can express and discuss their impression of the experience.

\subsection{The Squeezables (1998-1999)}

In the Squeezables project, I attempted to add the concept of musical networks to my initial interest in gestural controllers and constructionist education. The goal of the project was to allow a group of players, novices and proficient musicians, to interdependently collaborate in constructing a 
meaningful musical composition using unconventional expressive gestures. The instrument consisted of six squeezable and retractable gel balls mounted on a small podium, which players could simultaneously squeeze and pull to manipulate a set of low- and high-level musical percepts. The combination of pulling and squeezing allowed players to utilise familiar and expressive gestures and to control multiple synchronous and continuous musical parameters. Several materials were tested and for the final prototype, soft gel balls were chosen, which proved to be robust and responsive, providing a sense of force feedback control that derived from the elastic qualities of the gel. Buried inside each ball was a $0.5-\mathrm{x}-2.0-\mathrm{cm}$ plastic block covered with five pressure sensors, protected from the gel by an elastic membrane. The analog pressure values from these sensors were transmitted to a digitiser and converted to

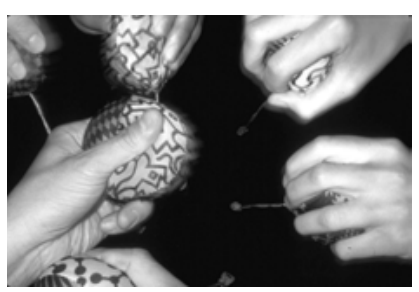

Fig. 2. Three networked players play The Squeezables MIDI. Pulling gestures were sensed by six variable resistors installed under the table. An elastic band connected to each ball added opposing force to the pulling gesture, helping to retract the balls back onto the tabletop (Fig. 2).

In an effort to evaluate the high-level algorithms in the instrument, a number of straightforward mappings were designed to control relatively low-level musical parameters. For example, one of the balls formed a one-to-one connection between squeezing and pulling gestures to the modulation rate and range of two low-frequency oscillators, respectively. For other balls higher-level algorithms were developed to control percepts such as contour and stability. For example, pulling and squeezing gestures of the "Arpeggiator" ball controlled a combination of musical parameters including tempo, pitch commonality, dissonance and rhythmic variation, so that the more the ball was squeezed and pulled, the more unstable an arpeggiated sequence became. To facilitate a coherent hierarchical interconnected interaction, the balls were divided into five accompaniment balls and one melody soloist. The five accompaniment balls provided players with autonomous control - no input from the other balls influenced their output. However, these balls' output was mapped not only to the accompaniment parameters but also to transform the sound of the "melody" ball. While pulling the "melody" ball manipulated its own contour so that the higher it was pulled, the higher the melodic curve became. The actual pitches, as well as the MIDI velocity, duration and pan values, were determined by the level of pulling and squeezing of the accompaniment balls. This allowed the accompaniment balls to "shape" the character of the melody while maintaining a comprehensive scheme of interaction among themselves.

\section{4}


To experiment with these mappings I composed a short piece for three players. The piece, which was featured in Ars Electronica $2000^{8}$, starts with a high-level of instability and builds gradually towards a repetitive rhythmic peak. Special notation was created for the piece - two continuous graphs were assigned to each one of the six balls. One graph indicated the level of squeezing over time and the other indicated the level of pulling. The process of writing and performing the piece served as a useful tool for evaluating the mapping and sensing techniques used. In addition, discussions were held with novices and professionals who played the instrument. In general, children and novices were more inclined to prefer playing the balls that provided high-level control such as contour and stability. They often stated that these balls allowed them to be more expressive and less analytical. Proficient musicians, on the other hand, often found the high-level control somewhat frustrating, because it did not provide them with direct and precise access to specific desired parameters. Some experts complained that their personal interpretation of the high-level controllers for stability differed from the one implemented in designing the instrument. Both novices and professional players found the multiple-channel synchronous control expressive and challenging and the pulling and squeezing gestures comfortable and intuitive.

These gestures allowed delicate and easily learned control of many simultaneous parameters, which was especially compelling for children and novices. The organic and responsive nature of the balls was one of the features mentioned as contributing to this expressive experience. When asked about the interdependent networked connections, one melody ball player described her experience as a constant state of trying to expect the unexpected. To another player, the experience felt like controlling an entity with a life of its own. In a manner similar to chamber music group interaction, body and facial gestures served an important role in coordinating the accompaniment players' gestures and establishing an effective outcome. Such collaborations turned out to be especially compelling for children, who found the accompaniment balls conducive to social interaction, intuitive and easy to play with. Some complaints were made, however, regarding the difficulty for individual accompaniment players to create their own musical phrases without being constantly subjected to interdependent transformation from the group. Other criticism addressed the lack of discrete input, which prevented players from generating and controlling specific musical events in detail. 


\subsection{The Musical Fireflies (1999-2000)}

The Musical Firefly project was designed to address some of the weaknesses in the Squeezables. In particular, it aimed to facilitate a more discrete and autonomous interaction that would allow for clearer interaction schemes and more focused constructionist-learning goals. The project attempted to provide players with expressive hands-on experiences that can be easily transformed into an analytical and formal exploration of music and mathematics. Through simple tapping gestures players could input rhythmic patterns and embellish them in real-time by adding multiple rhythmic layers. This functionality provided players with figural and formal familiarisation with musical concepts such as accents, beats, patterns, and timbre. During the multi-player interaction, a wireless network was formed between Fireflies, which allowed players to synchronise patterns and trade instrument sounds. This interactive group experience was designed to lead to deeper internalisation of advanced musical concepts such as the correlation between monorhythmic and polyrhythmic structures. Access to and manipulation of LOGO code for customising the controllers provided an introduction to MIDI programming and electronic sound. Advanced players could, therefore, deepen their learning experience by reprogramming the controllers and adjusting their functionality to match personal musical interests and abilities.

The 3D printed Musical Firefly's case was designed to be held by two hands while thumb-tapping two top-mounted buttons. Signals from the buttons were sent to an embedded "Cricket" Microchip PIC microprocessor. An infrared communication port allowed for communication with other Fireflies as well as for downloading LOGO based application programs. The

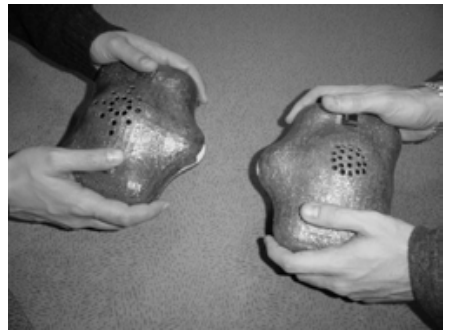

Fig. 3. Two players interact with each other with the Musical Fireflies

players converted numerical patterns into rhythmical structures, and the Multi Player Mode, where collaboration with other players enhanced the basic rhythmic structures into polyrhythmic compositions (Fig. 3). In Single Player played rhythmic patterns were converted into musical messages using Cricket LOGO general MIDI commands and sent through the Cricket's serial bus port to the MidiBoat - a small General Midi circuit that supported up to 16 polyphonic channels, 128 melodic timbres and 128 percussive timbres. The audio from the MidiBoat was then sent to the topmounted speaker.

Interaction with the Musical Fireflies occurred in two distinct and sequential modes - the Single Player Mode, where

\section{6}


Mode, players could trigger and play with two default percussive sounds. The left button triggered accented notes and the right button triggered non-accented notes. The patterns of accented and non-accented notes were recorded and after two seconds of inactivity, played back in a loop, using an adjustable default tempo. This activity provided players with a tangible manner of entering and listening to the rhythmical output of any numerical pattern they envisioned, leading to an immediate conceptualisation of the mathematical-rhythmical correlation. For example, Figure 4 depicts the playing of the numerical pattern 43522 :

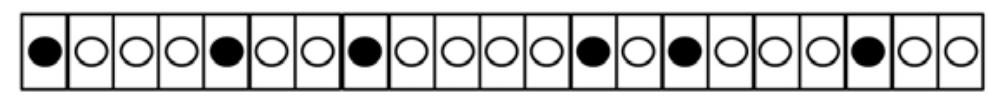

Fig. 4. A pattern of accented and non-accented notes as played by the Musical Fireflies. $\bullet=$ Accented note played by the left button; $0=$ non accented note played by the right button

During playback, players could enter a second layer of accented and nonaccented notes in real-time, using a different timbre. Each tap on a button triggered a note aloud and recorded its quantised position so that the pattern became part of the rhythmic loop. Pressing both buttons simultaneously at any point stopped the playback and allowed the player to enter a different pattern. In Multi Player Mode, when two loop playing Fireflies "saw" each other (i.e., when their infrared signals were exchanged), they automatically synchronised their rhythmic patterns. (A similar interaction occurs when the Firefly insects synchronise their light pulses to communicate in the dark). This activity provided participants with a richer, more complex rhythmical composition and allowed for an interactive introduction to polyrhythm. Figure 5 depicts how a 7 beat pattern played by one Firefly and a 4 beat pattern played by another diverge and converge as the patterns go in and out of phase every 28 beats, the smallest common denominator:

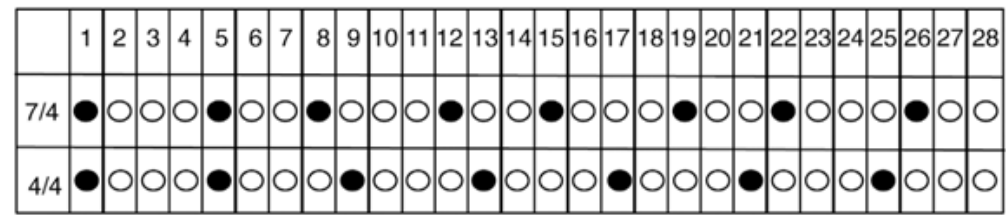

Fig. 5. Two patterns (7/4 and 4/4) played by two Fireflies divergence and convergence as they go in and out of phase every 28 beats 
While the two Fireflies were synchronised, players could also initiate a "Timbre Trade" in which instrument sounds were exchanged between the devices. Pressing either the left or right button traded both layers of the accented or non-accented timbres respectively. Each Firefly continued to play its original pattern using the new received timbre. This interaction provided players with a higher-level of musical abstraction as they separated the rhythmical aspect of the beat from the timbre in which it was played. Because the Fireflies network became richer after the interaction (i.e., each instrument contained four different timbres) the system encouraged collaborative play where players were motivated by trading, collecting and playing games by sending and receiving different timbres from their peers.

Observations of play sessions with the Musical Fireflies have been conducted followed by discussions with the players. Participants were asked about the expressive and the educational aspects of the session as well as for their suggestions for improvements. A software version of the application was prepared and tested. Both novices and experienced users found the concrete aspects of playing with a physical object compelling in comparison with the graphical user interface of the software version, mentioning the unmediated connection that was formed with the instrument as contributing to the creation of personal connection with their music they created. Listening to the music from distinct physical sources also helped players to follow the interaction in a more coherent manner in comparison to listening to computer speakers. The observations and interviews also led to the identification of points for improvement and future work. For example, it was clear that the focus on a specific constructionist learning activity hampered the open-ended expressive gestural interaction goal of the project. Moreover, the simple interaction using only two discrete buttons and the low-quality MIDI sounds led to a disappointing musical outcome, consisting mostly of monotonous interlocking clicks with no pitch, time-based rhythmic values, rests, or continuous transformation. The network interaction in multi-user mode, while effective for learning, did not provide a satisfactory collaborative experience. The restricted interconnectivity of the system, where discrete timbre-trading was the only interpersonal act, did not provide long-lasting rich play value and led players to lose interest in the interaction after a few trades. In addition, due to the limitations imposed by the line-of-sight infrared communication, the application only allowed for synchronisation and timbre trading between two players at a time. Many interviewees expressed their wishes to interact and collaborate in larger groups comprised of several simultaneous players.

\section{8}




\subsection{The Beatbugs / "Nerve" (2001-2003)}

For the Beatbug project, new hardware and software applications were developed in an effort to address the weaknesses identified in the Musical Fireflies. The binary buttons were replaced with a piezo electric sensor that could sense hit strength, providing more expressive physical interaction through large full-arm drumming gestures. The single user application was enhanced to record rhythmic values, rests, pitches, and amplitudes, allowing for more versatile and expressive musical input. Two new bend sensors were added to the design, allowing players to continuously modify and transform the recorded musical phrases using low- and high-level transformation algorithms (Fig. 6). In addition, the embedded MIDIBoat was replaced with a high-quality software synthesiser, which significantly enhanced sound quality and versatility. Several important enhancements were also made to improve the multi-user collaborative interaction. The network was enhanced to support up to eight simultaneous Beatbugs, while coloured LEDs were installed in each Beatbug to help convey complex multi-user interactions in a visual manner. The interpersonal application was improved to provide longer lasting collaborative interactions, allowing players to continuously develop each other's music by bending and manipulating the Beatbug antennae. In order to support these improvements, the new Beatbugs communicated with each other through wires via a central computer system, which was titled the "Nerve Center". To showcase the improved system, a musical composition was composed, titled "Nerve", which was presented in workshops and concerts as part of the Tod Machover's Toy Symphony project.

In an effort to provide a familiar and fun interface for children and novices, the "Nerve" Beatbug was designed as a bug, having a speaker for a mouth, two bend-sensors for antennae, and a velocity-sensitive piezoelectric sensor on its back. White and coloured LEDs mounted in its translucent shell provided visual feedback when hit or played through. An embedded Microchip PIC microcontroller was responsible for reading input from the sensors, controlling the LEDs, and communicating with the central system via tail-like cable that carried MIDI, trigger, audio, and power. The piezo electric sensor measured when and how hard it was hit,

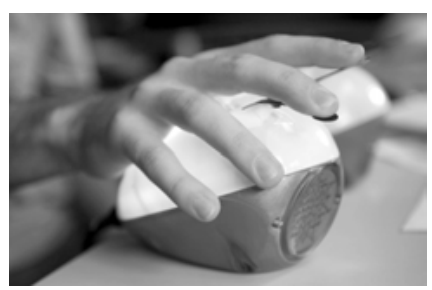

Fig. 6. Manipulating the Beatbugs antennae while the two antennae allowed for subtle control over different aspects of the sound. Bending the antennae caused a proportional change in the colour of three LED clusters, and a ring of white LEDs flashed each time the bug was hit, providing additional visual feedback 
to the player and audience. The embedded processor was responsible for operating the sensors and LEDs, while the central computer system controlled the actual musical interactions and behaviours. The "brain" of the system was written in Max/MSP environment. Controlling all of the behaviour from the central computer made it easy to quickly experiment with a broad range of interaction schemes. Similarly, sound synthesis occurring on the central computer and played through the corresponding Beatbug's speaker, provided high quality sound with an embedded, self-contained feel. For the software synthesiser, 'Reason' by Propellerhead was chosen, providing a broad palette of timbres and continuous control over multiple sound parameters. Up to eight Beatbugs could be connected to one central rack, which consisted mostly of standard off-the-shelf equipment including an audio interface, a MIDI interfaces, an 8-channel amplifier, and a mixer. The only non-standard device in the system was a custom patch box, which provided power to the bugs and converted the 10-pin connector in each cable to MIDI in, MIDI out, trigger, and audio in (Fig. 7).

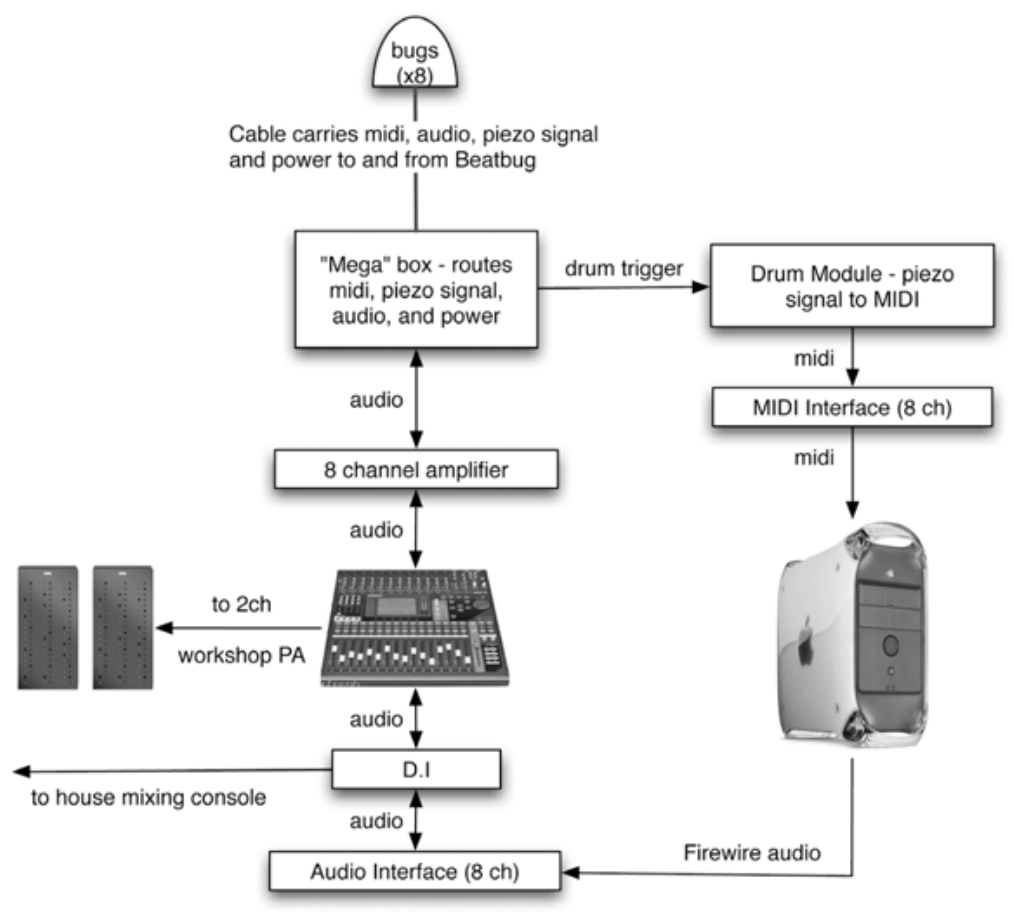

Fig. 7. The Nerve Beatbug system's schematics 
Similarly to the Musical Fireflies, players interacted with the "Nerve" Beatbug in two distinct modes - Single Player Mode, and Multi Player Mode. In Single Player Mode, each player could enter a short rhythmic pattern over a predefined metronome beat. The system automatically played back the recorded pattern in a loop through the corresponding Beatbug's speaker. A quantisation algorithm pushed the notes towards the closest quarter, eighth or triplet note. While the entered pattern was playing back, the player could manipulate the pattern by bending the two antennae. The left antenna continuously transformed the pitch and timbre using a variety of predefined scales and audio effects. The right antenna added rhythmic ornamentation to the pattern by controlling the values, length, accentuation, and feedback level of a delay line. The goal of these transformation algorithms was to allow players to modify the pattern but to keep the feel of the original motif, supporting the "motif-and-variation" nature of the interaction. In Multi Player Mode players could form large-scale collaborative compositions by interdependently sharing and continuously developing each other's motifs. Each Beatbug player could play a rhythmic motif that was then automatically sent through the stochastic computerised "Nerve Center" to another player in the group. The receiving player could decide whether to further develop the received motif (by continuously manipulating pitch, timbre, and rhythmic elements with the two bend sensor antennae) or to keep the motif in his or hers personal bug (by entering and sending a newly generated motifs to a different random player in the group). The antennae transformations were recorded and layered in each cycle until a new pattern was entered. The tension between the system's stochastic routing scheme and the players' improvised real-time decisions led to an interdependent, dynamic, and constantly evolving musical outcome. In a different section of Multi Player Mode, after all players entered their patterns, the system awaited a series of simultaneous hits by all players that led to random segmentation of the participants to sub-groups, allowing players to interdependently collaborate with a gradually

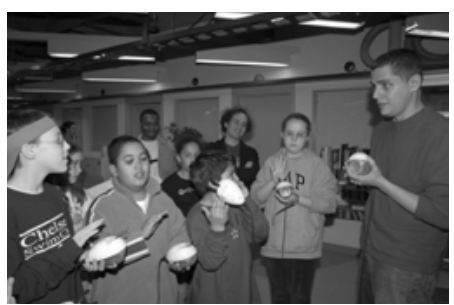

Fig. 8. A Beatbug workshop at MIT Media Lab growing number of co-players. ${ }^{9}$

During 2002-2003 the "Nerve" Beatbugs were featured in workshops and concerts in Berlin, Dublin, Glasgow, Boston and New York in collaboration with local symphonies and educational programs (Fig. 8). During each week-long workshop, children and orchestra members were introduced to

9 See a video clip of the interaction as performed in concert at <http: / / www.cc.gatech. edu / gilwein/videos / Glasgow\%20-\%20Concert.mov>. 
the Beatbugs, explored the system, and rehearsed towards a public concert. The workshops also featured a new constructionist pedagogy developed in collaboration with Kevin Jennings. The pedagogy was designed to allow players to physically create and phrase rhythmic patterns and transform them by employing melodic, timbral, and rhythmic contours. The balance among aural, kinesthetic and social modalities provided the children with a rich and highly immersive musical environment. A report by Project Zero from Harvard's Education School said that "[the project] provided an overwhelmingly positive experience either from the musical, social and personal standpoint... the experience provided a good foundation on which to build one's musicianship, social skills, self-confidence, and general learning dispositions focusing, listening, and practicing."

Several problems and areas for improvement became apparent as well. The musical mappings in Single Player Mode, although more versatile than in the Musical Fireflies, were still limited and unsatisfactory for many proficient musicians, who expressed their interest in creating and manipulating more advanced and non-quantised melodic and harmonic musical content. Novices too showed interest in controlling more sophisticated musical mate-

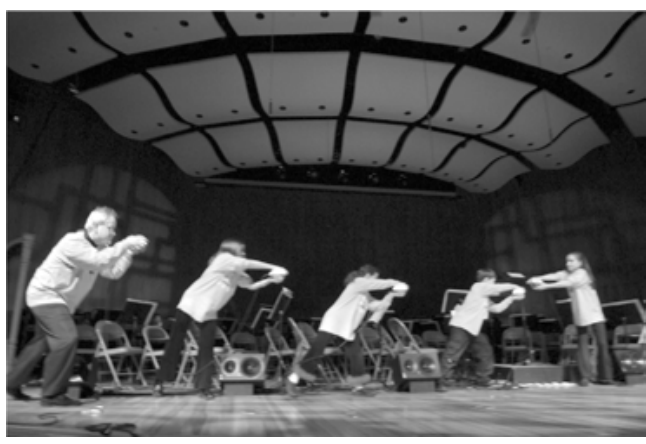

Fig. 9. Large play gestures in a "Nerve" concert, Cambridge, MA rial even if they could not create it themselves. In multiplayer interactions, the velocity sensing piezoelectric sensor and the large scale of the system encouraged players to use wide playing gestures and expressively point to indicate their actions to each other and to the audiences (Fig. 9). However, while these large gestures brought elements of visual expression and excitement to the performance, they were not sensed by the central system and therefore did not have audible consequences. In terms of hardware, it was clear that the central system was too large and complex, and that the 18-unit rack was not easily portable. An additional hardware weakness was the durability of the bend sensor antennae, which proved to be fragile, especially when large groups of energetic children experimented with the system during week-long workshops. 


\section{5 iltur (2003-2005)}

The iltur project utilised an improved version of the Beatbug controllers, which were enhanced both in hardware and software in an effort to address the weaknesses observed in Nerve. Hardware improvements included replacing the unreliable bend sensors with robust Hall effect sensors, installing 2D accelerometers to sense larger and more expressive arm gestures, and reducing the size and complexity of the system. The software was rewritten to address users' requests to control and manipulate advanced melodic and harmonic content in a more expressive and gestural manner. The new application supported interaction between Beatbug players and proficient musicians, allowing Beatbug players to record live input from MIDI and acoustic instruments and to respond by transforming the recorded material gesturally, creating motif-and-variation call-and-response routines on the fly. The central computer host was programmed to analyse MIDI and audio signals and to allow Beatbug players to personalise the analysed material using a variety of transformation algorithms. Capturing and personalising richer musical content through expressive gestures gave Beatbug players the opportunity to create a more sophisticated musical outcome, while forming elaborate musical dialogs with their peers.

The main hardware improvement in the iltur Beatbugs was the addition of the $2 \mathrm{D}$ accelerometers. The accelerometers were used to sense tilting and shaking gestures, providing the central system with information regarding players' large arm movements. Hardware improvements were also made in an effort to make the antennae more robust, utilising Hall effect sensors and magnets mounted under the antennae. This electromagnetic sensing method proved to be robust and effective, although it provided lower bending resolution in comparison with the original resistance-based bend sensors. Other hardware improvements addressed the system size and portability. As opposed to the complex 18-unit rack Nerve system, the new iltur system, utilised a laptop instead of a desktop, a software mixer instead of a physical one, and no MIDI drum controller, as audio from the piezoelectric sensors was captured directly through an audio interface. The system, therefore, was housed in a small 6-unit rack (Fig. 10).

Play gestures and interaction in iltur were modified to allow for recording, triggering, and manipulation of MIDI and audio in real-time. Recording was conducted by simultaneously bending both antennae while tapping the Beatbug. The system then segmented the recorded phrases, looking for sections of silence in the MIDI and/or audio buffers. The audio Beatbugs were programmed to detect onset notes, pitches, and amplitudes in real-time. The analysis algorithm was optimised for brass instruments and was used successfully with instruments such as trumpet, trombone and saxophone. Onset 


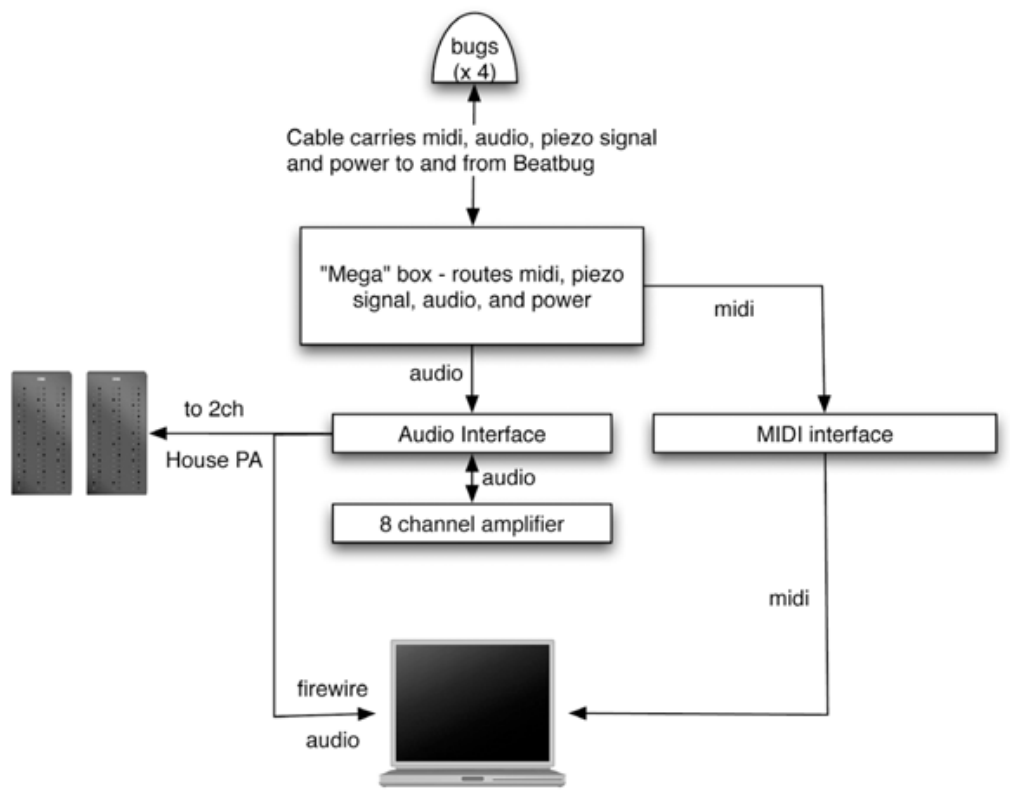

Fig. 10. The iltur Beatbug system's schematics

identification and segmentation of MIDI was trivial due to the discrete nature of the MIDI protocol. After the system recorded and segmented the captured musical input, players could immediately trigger the recorded phrase by tapping the Beatbug again. Hit velocities were mapped to different segments in the phrase, allowing players to rearrange the recorded motifs. Two synthesis methods - Wavetable Synthesis and Granular Synthesis - were used for retriggering audio. The Wavetable technique provided close resemblance to the sound of original recording but suffered from noise artifacts during continuous transformations. Granular Synthesis, on the other hand, provided harsher sounds in comparison to the original recording but allowed for smoother continuous transformation. A number of different mapping schemes were experimented with for antennae bending and accelerometer-based gestures. Some of these algorithms utilised direct mappings between continuous gestures and fundamental musical aspects such as pitch, volume and tempo. Other mapping approaches allowed for the manipulation of higher-level musical percepts such as melodic similarity or rhythmic density. Shaking gestures were most successful when mapped to control vibrato and tremolo effects, while antennae manipulations were effective in controlling pitch. When interacting with a MIDI instrument, Beatbug players could also trigger the recorded

\section{4}


motif in inversion and retrograde by tapping the Beatbug while bending the left or right antennae, respectively. The audio Beatbugs allowed players to control transformations such as pitch bending, speed alteration, and filtration, through a combination of bending, tilting, and hitting gestures. During group interaction, players could trade their motifs by simultaneously hitting the Beatbug while bending one of the antennae. Receiving players could then further transform the phrase and send it back to their peers. In comparison to the random involuntarily routing scheme in Nerve, iltur players could trade their motifs only when simultaneously agreeing to synchronise their gestures. Three Jazz compositions were written for the iltur system and performed in cities such as Atlanta, San Diego, Miami, Vancouver, and

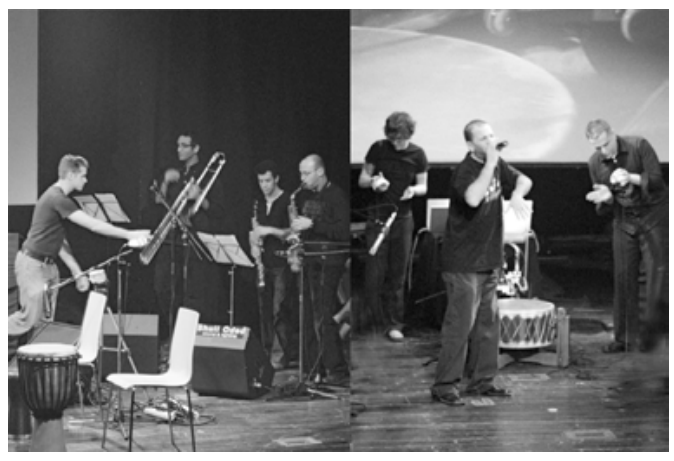

Fig. 11. iltur 3 audio Beatbug players interact with a brass section (left) and a hip-hop vocalist (right) in Jerusalem, Israel

Jerusalem. iltur 1 featured MIDI interaction, iltur 2 focused on audio transformation and manipulation, and iltur 3 introduced group interaction and motif trading. Voice manipulation experimentations were also conducted, allowing Beatbug players to interact with a hip-hop vocalist. ${ }^{10}$

Observations of and discussion with iltur players led to a number of findings regarding the improved Beatbug functionalities. For example, it was clear the iltur Beatbugs were more effective than the Nerve Beatbugs in providing richer musical experiences for individuals through a larger set of expressive gestures and more complex melodic and harmonic transformations. The new application also led to more meaningful and versatile collaborations between novices and professional musicians. Both players and audiences perceived the new accelerometer-based gestures as intuitive, expressive, and visually compelling. However, the introduction of gesture combinations (such as hitting the Beatbug while bending the antenna) was problematic for novices and children, who found it physically and mentally challenging. Novices and children also found the higher-level transformation algorithms (such as musical density and stability) less intuitive to control and preferred the simple and predictable one-to-one mappings between gestures and low-level musical

10 See videos at <http: / / www.cc.gatech.edu/ gilwein/iltur.htm>. 
aspects. More proficient musicians, on the other hand, preferred to interact with the high-level musical operations, stating that these encouraged them to concentrate on the correlation between their actions and the musical output.

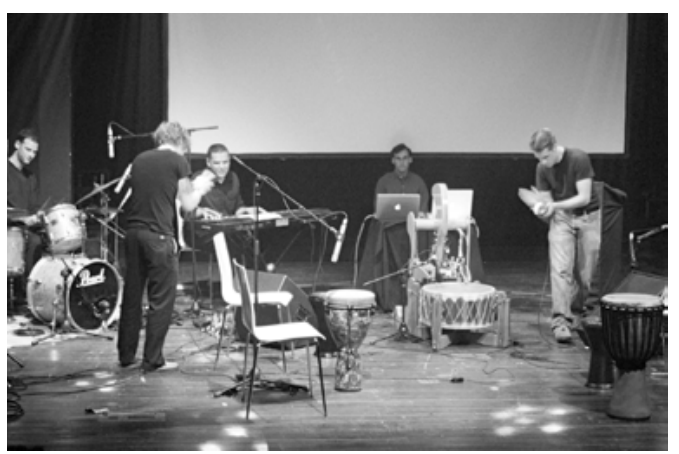

Fig. 12. Interaction between two iltur 3 MIDI Beatbug players In general, the effectiveness of the experience was closely related to the musical and harmonic context of the compositions. Due to segmentation and audio stretching, in a harmonically structured composition it was difficult for players to improvise while following the harmonic progression. Many players, therefore, preferred free musical structures, stating that open-ended experience posed less boundaries and allowed more creativity and expression.

\subsection{Haile (2004-2007)}

The instruments and controllers discussed above explored different ways in which meaningful embodiment of technology can enhance the musical experience by facilitating new expressive gestures, networked group collaborations and constructionist learning. Although these projects provided satisfying results, the instruments were limited by the electronic reproduction and amplification of sound through speakers, which did capture the richness of acoustic sound. My most recent project - an interactive robotic percussionist named Haile - addressed this limitation by utilising a mechanical apparatus that converts digital musical instructions into acoustic and physical generation of sound. Haile was developed in an effort to bring together the advantages of computational power with the expression and richness of creating acoustic sound using physical and visual gestures.

The project aimed to combine that are not possible by humans with rich sound and visual gestures that cannot be reproduced by speakers in an effort to facilitate new musical experiences, and new music, that cannot be conceived by acoustic or means.

As part of the project, a robotic percussionist that listened to and analysed live musical input in real-time and reacted by generating relevant, but

\section{6}


at times surprising, acoustic responses was developed. The project posed challenges in areas such as perception modeling, mechanics, and interaction design. In perception, the main challenge was to implement models for lowand high-level musical percepts, allowing the robot to develop a meaningful representation of the music it listened to. In mechanics the challenge was to develop a dexterous robotic apparatus that would translate perceptually based performance algorithms into a rich acoustic and visually informative performance. In interaction design, our aim was to develop performance algorithms that would enable the robot to collaborate with human players in a meaningful and intuitive manner, using transformative and generative methods both sequentially and synchronously.

In order to support familiar interactions with human players, Haile's design is anthropomorphic, utilising two percussive arms that can move to different locations and strike with varying velocities (Fig. 13). The first prototype was designed to play a Native American Pow Wow drum - a multi player instrument that supported the collaborative nature of the project. For pitch-oriented applications, the robot was later adjusted to play a one-octave xylophone. In order to match the aesthetics of these musical instruments, Haile was constructed from wood using a $\mathrm{CnC}$ cutting machine. Metal joints were designed to allow shoulder and elbow movement as well as leg adjustability for different instrument heights. While attempting to create an organic look for the robot, it was also important that the technology was not completely hidden, so that co-players could see and understand the robot's operation. The mechanical apparatus was therefore left uncovered and LEDs were embedded on Haile's body, providing an additional representation of the mechanical actions. Haile's right arm was designed to play fast notes, while the left arm was designed to produce larger and more visible motions that produce louder sounds. Both arms could adjust the strikes sound in two manners: different pitches were achieved by striking the instruments in different locations, and volume was adjusted by hitting with varying velocities.

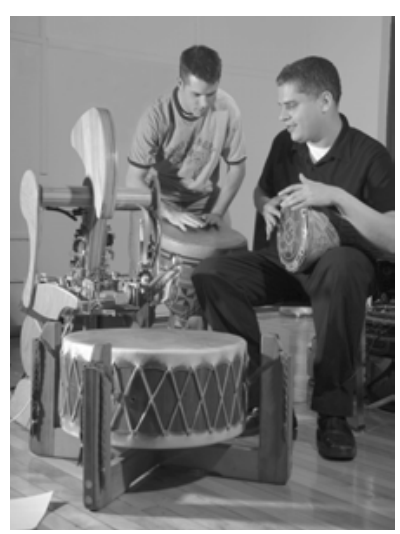

Fig. 13. Haile, the perceptual robotic percussionist, listens to and interacts with a human player To move to different vertical positions, each arm employed a linear slide, a belt, a pulley system, and a potentiometer to provide feedback. Unlike robotic drumming systems that allow hits at only a few discrete locations, Haile's arms moved continuously over a distance of 10 
inches (movement timing is $250 \mathrm{~ms}$. from end to end). The right arm's striking mechanism was loosely based on a piano hammer action and consisted of a solenoid driven device and a return spring. The right arm stroked at a maximum speed of $15 \mathrm{~Hz}$, faster than the left arm's maximum speed of 11 $\mathrm{Hz}$. However, the right arm did generate a wide dynamic range or provided easily noticeable visual cues, which limited Haile's expression and interaction potential. The left arm was designed to address these shortcomings, using larger visual movements, and a more powerful and sophisticated hitting mechanism.

The first phase of the project aimed at facilitating rhythmic collaboration between human drummers and Haile, addressing aspects such as rhythmic perception, improvisation, and interaction design. Perceptual models were developed for low- and high-level rhythmic percepts, from beat and density analysis, to rhythmic stability and similarity perception. Some relatively lowlevel perceptual modules included beat analysis, where domain detection was followed by autocorrelation of tempo and phase, and density analysis, where we looked at the number of note onsets per time unit to represent the density of the rhythmic structure. Higher-level rhythmic analysis modules were also developed for percepts such as rhythmic stability, based on research by Desain, et al. ${ }^{11}$, and rhythmic similarity based on Tanguiane's survey ${ }^{12}$. The stability model calculated the relationship between pairs of adjacent note durations, rated according to their perceptual expectancy based on three main criteria: perfect integer relationships were favoured, ratios had inherent expectancies (i.e., 1:2 was favoured to $1: 3$ and 3:1 was favoured to $1: 3$ ), and durations of 0.6 seconds were preferred. The similarity rating was derived from Tanguiane's binary representation, where two rhythms are first quantised, and then given a score based on the number of note onset overlaps and near-overlaps.

The main challenge in designing the rhythmic interaction with Haile was to implement the perceptual modules in a manner that would lead to an inspiring human-machine collaboration. The approach taken was based on a theory of interdependent group interaction in interconnected musical networks. At the core of this theory is a categorisation of collaborative musical interactions in networks of artificial and live musicians based on sequential and synchronous operations with centralised and decentralised control schemes. Based on this framework, six interaction modes were developed: Imitation, Stochastic Transformation, Perceptual Transformation, Beat Detection, Simple Accompaniment, and Perceptual Accompaniment. These interaction modes utilised different perceptual modules and were embedded

11 Desain et al. 2002

12 Tanguiane 1993

\section{8}


in different combinations in interactive compositions and educational activities. In the first mode, Imitation, Haile merely repeated what it heard based on its low-level onset, pitch, and amplitude perception modules. Players could play a rhythm and after a couple of seconds of inactivity Haile imitated it in a sequential call-and-response manner. Haile used one of the arms to play lower pitches close to the drumhead centre and the other arm to play higher pitches close to the rim. In the second mode, Stochastic Transformation, Haile improvised in a call-and-response manner based on players' input. Here, the robot stochastically divided, multiplied, or skipped certain beats in the input rhythm, creating variations of users' rhythmic motifs while keeping their original feel. Different transformation coefficients were adjusted manually or automatically to control the level of similarity between humans' motifs and Haile's responses. In the Perceptual Transformation mode, Haile analysed the stability level of users' rhythms, and responded by choosing and playing other rhythms that had similar levels of stability to the original input. In this mode Haile automatically responded after a specified phrase length. Imitation, Stochastic Transformation, and Perceptual Transformation were all sequential interaction modes that formed decentralised call-and-response routines between human players and the robot. Beat Detection and Simple Accompaniment modes, on the other hand, allowed synchronous interaction where humans played simultaneously with Haile. In Beat Detection mode, Haile tracked the tempo and beat of the input rhythm using complex domain detection function and autocorrelation, which led to continuously refined assumptions of tempo and phase. A simpler, yet effective, synchronous interaction mode was Simple Accompaniment, where Haile played pre-recorded MIDI files so that players could interact with it by playing their own rhythms or by modifying elements such as drumhead pressure to modulate and transform Haile's timbres in real-time. This synchronous centralised mode allowed composers to feature their structured compositions in a manner that was not susceptible to algorithmic transformation or significant user input. The Simple Accompaniment mode was also useful for sections of synchronised unisons where human players and Haile played together. Perhaps the most advanced mode of interaction was the Perceptual Accompaniment mode, which combined synchronous, sequential, centralised and decentralised operations. Here, Haile played simultaneously with human players while listening to and analysing their input. It then created local call-and-response interactions with different players, based on its perceptual analysis. In this mode amplitude and density perceptual modules were utilised - while Haile played short looped sequences (captured during the Imitation and Stochastic Transformation modes) it also listened to and analysed the amplitude and density curves of human playing. It then modified its looped sequence, based on the amplitude and density coefficients of the human players. When the 
rhythmic input from human players was dense, Haile played sparsely, providing only the strong beats and allowing humans to perform denser solos. When humans played sparsely, on the other hand, Haile improvised using dense rhythms that were based on stochastic and perceptual transformations. Haile also responded in direct relationship to the amplitude of human players so that the louder humans played, the stronger Haile played to accommodate the human dynamics, and vice versa. ${ }^{13}$

As a creative outcome for these interactive applications, two compositions were written for the system, each utilised a different set of perceptual and interaction modules. The first composition, titled Pow, was written for one or two human players and a one-armed robotic percussionist. It served as test

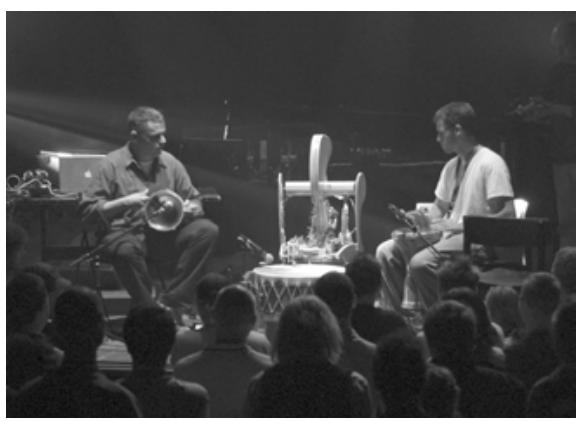

Fig. 14. A performance of Jam'aa in Odense, Denmark case for Haile's early mechanical, perceptual, and interaction modules. The second composition, titled Jam'aa ("gathering" in Arabic), built on the unique communal nature of the Middle Eastern percussion ensemble, attempting to enrich its improvisational nature, calland-response routines, and virtuoso solos with algorithmic transformation and humanrobotic interactions (Fig. 14). Jam'aa, was commissioned by Hamaabada Art Centre In Jerusalem, and later performed in invited and juried concerts in France, Germany, Denmark, and the United States. ${ }^{14}$

As part of our effort to expand the exploration of robotic musicianship into pitch and melody, Haile was later adapted to play a pitch-based mallet instrument. A one-octave xylophone was built for this purpose to accommodate Haile's mechanical design - the left arm covered a range of 5 keys while the right arm, whose vertical range was extendable, covered a range of 7 keys. (Fig. 15). Following the idiom "listen like a human, improvise like a machine", computational models for melodic similarity were developed ("listen like a human") as the fit function of a genetic algorithm based improvisation engine ("improvise like a machine"). The algorithmic responses were based on the analysed input as well as on internalised knowledge of contextually relevant

13 See a video excerpts of some of the interaction modes at <http:/ /www.cc.gatech. edu/ gilwein/Haile.htm>.

14 See a video excerpts from Jam'aa at <http://coa.gatech.edu/ gil/RoboraveShort. mov>.

\section{0}


material. The algorithm fragmented MIDI and audio input to short phrases. It then attempted to find a "fit" response by evolving a pre-stored human-generated population of phrases using a variety of mutation and crossover functions over a variable number of generations. At each generation, the evolved phrases were evaluated by a fitness function that measured similarity to the input phrase, and the least fit phrases in the database

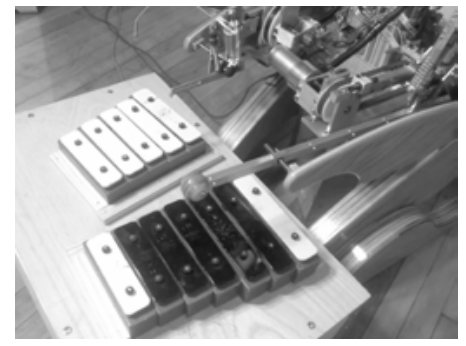

Fig. 15. Haile's adaptation for xylophone are replaced by members of the next generation. A unique aspect in this design was the reliance on a pre-recorded human-generated phrase set that evolved over a limited number of generations. This allowed musical elements from the original phrases to mix with elements of real-time input to create hybrid, and at times unpredictable, responses for each given input melody. Two compositions were written for the system and performed in concerts in

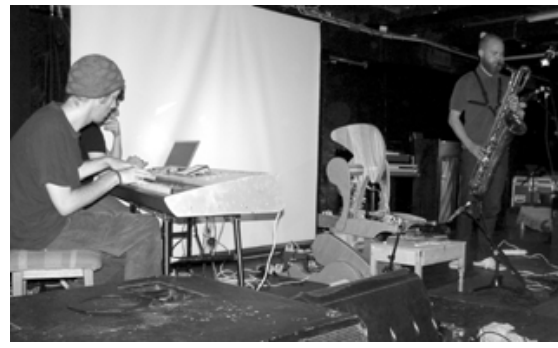

Fig. 16. A performance of Suobod in Copenhagen, Denmark
Atlanta and Copenhagen. In the first piece, titled "Svobod", a piano and a saxophone player freely improvised with a semiautonomous robot (Fig. 16). The second piece, titled "iltur for Haile", involved a tonal musical structure utilising genetically driven and non-genetically driven interaction schemes, as the robot performed autonomously with a jazz quartet. ${ }^{15}$

15 See a video clip of iltur for Haile at <http://www.coa.gatech.edu/ gil/iltur4haile. mov>. 


\section{References}

Aimi, Roberto (2002): New Expressive Percussion Instruments, M.S. Thesis, MIT Media Laboratory.

Aimi, Roberto/Young, Diana (2004): »A New Beatbug: Revisions, Simplifications, and New Directions«. In: Proceedings of the 2004 International Computer Music Conference, San Francisco: International Computer Music Association.

Bamberger, Jeanne (2000): Developing Musical Intuitions, New York: Oxford University Press.

Barbosa, Alvaro (2003): »Displaced Soundscapes: A Survey of Network Systems for Music and Sonic Art Creation«. Leonardo Music Journal 13, 53-59.

Bischoff, John/Gold, Rich/Horton, Jim (1978): »Microcomputer Network Music«. Computer Music Journal 2(3), 24-19.

Buchla, Don (2005): »A history of Buchla’s musical instruments«. In: Sidney Fels/ Tina Blaine (Eds.), New Interfaces for Musical Expression, NIME-05, Proceedings, Vancouver: University of British Columbia, Media and Graphics Interdisciplinary Center (MAGIC), 1.

Cope, David (1996): Experiments in Music Intelligence, Madison WI: A-R Editions.

Cycling74 (2005): Max/MSP <http:/ / www.cycling74.com> (last access: October 2007).

Dannenberg, Roger B. (1985): »An On-line Algorithm for Real-Time Accompaniment«. In: William Buxton (Ed.), Proceedings of the 1984 International Computer Music Conference, San Francisco: International Computer Music Association, 193-198.

Dannenberg, Roger B./Brown, Ben/Zeglin, Garth/Lupish, Ron (2005): »McBlare: A Robotic Bagpipe Player«. In: Sidney Fels/Tina Blaine (Eds.), New Interfaces for Musical Expression, NIME-05, Proceedings, Vancouver: University of British Columbia, Media and Graphics Interdisciplinary Center (MAGIC), 80-84.

Desain, Peter/Honing, Henkjan (2002): »Rhythmic stability as explanation of category size $\ll$. In: Proceedings of the International Conference on Music Perception and Cognition, Sydney: UNSW, CD-Rom.

Feldmeier, Mark C./Malinowski, Mateusz/Paradiso, Joseph A. (2002): »Large Group Musical Interaction Using Disposable Wireless Motion Sensors«. In: Proceedings of the 2002 International Computer Music Conference, San Francisco: International Computer Music Association, 83-87.

Gardner, Howard (1983): Frames of Mind: The Theory of Multiple Intelligences, New York: Basic Books.

Gresham-Lancaster, Scot (1998): »The Aesthetics and History of the Hub: The Effects of Changing Technology on Network Computer Music«. Leonardo Music Journal 8, 39-44.

Jennings, Kevin (2003): »Toy Symphony: An International Music Technology Project for Children«. Music Education International 2, 3-21.

\section{2}


Jordà, Sergi (2002): »Afasia: The Ultimate Homeric One-man multimedia-band«. In: Eoin Brazil (Ed.), New Interfaces for Musical Expression, NIME-02, Proceedings, Dublin: Media Lab Europe, 132-137.

Levin, Golan (2001): »Dialtone: A Telesymphony«. Online available: <http:/ /www.flong. com/projects/telesymphony/> (last access: October 2007).

Lewis, George E. (2000): »Too Many Notes: Computers, Complexity and Culture in Voyager«. Leonardo Music Journal 10, 33-39.

Machover, Tod (1992): Hyperinstruments: A Progress Report 1987-1991, Cambridge: MIT Media Laboratory.

Machover, Tod (2004): »Shaping Minds Musically«. BT Technology Journal 22(4), $171-179$.

Martin, Fred/Mikhak, Bakhtiar/Silverman, Brian (2000): »MetaCricket: A designer's kit for making computational devices«. IBM Systems Journal 39, 3-4.

Mathews, Max V. (1991): »The Radio Baton and Conductor Program, or: Pitch, the Most Important and Least Expressive Part of Music«. Computer Music Journal 15(4), 37-46.

Nikitina, Svetlana (2004): »Toy Symphony Report«, Cambridge: Harvard University School of Education.

Pachet, Francois (2002a): »The continuator: Musical interaction with style«. In: International Computer Music Association (Ed.), Proceedings of the 2002 International Computer Music Conference. San Francisco: International Computer Music Association.

Papert, Seymour (1980): Children, Computers, and Powerful Ideas, New York: Basic Books.

Paradiso, Joseph A. (2004): »Wearable Wireless Sensing for Interactive Media«. In: Proceedings of the 1st International Workshop on Wearable and Implantable Body Sensor Networks, 2004, 30-31.

Patten, James/Recht, Benjamin/Ishii, Hiroshi (2002): »Audiopad: a tag-based interface for musical performance«. In: Eoin Brazil (Ed.), New Interfaces for Musical Expression, NIME-02, Proceedings, Dublin: Media Lab Europe, 11-16.

Proceedings of International Conferences on New Interfaces for Musical Expression (NIME), <http: / /www.nime.org/> (last access: October 2007).

Resnick, Mitchel/Martin, Fred/Sargent, Randy/Silverman, Brian (1996): »Programmable Bricks: Toys to Think With«. IBM Systems Journal 35(3-4), 443-452.

Rowe, Robert (2004): Machine Musicianship, Cambridge: MIT Press.

Scheirer, Eric D. (1998): »Tempo and beat analysis of acoustic musical signals«. In: Journal of the Acoustical Society of America 103(1), 588-601.

Singer, Eric/Feddersen, Jeff/Redmon, Chad/Bowen, Bil (2004): »LEMUR's Musical Robots«. In: Yoichi Nagashima/Michael J. Lyons (Eds.), New Interfaces for Musical Expression, NIME-04, Proceedings, Hamamatsu: Shizuoka University of Art and Culture, $181-184$. 
Smith, Joshua R./Strickon, Josh (1998): »The MIDIBoat«. Online available: <http:// web.media.mit.edu/ jrs/minimidi/> (last access: October 2007).

Steiner, Nyle A. (2004): »The Electronic Valve Instrument (EVI), an electronic musical wind controller for playing synthesizers«. Journal of the Acoustical Society of America 115(5), 2451-2451.

Takanishi, Atsuo/Maeda, Maki (1998): »Development of Anthropomorphic Flutist Robot WF-3RIV«. In: Proceedings of the 1984 International Computer Music Conference, San Francisco: International Computer Music Association, 328-331.

Tanguiane, Andranick S. (1993): Artificial Perception and Music Recognition, New York: Springer.

Theremin, Leon (1996): »The Design of a Musical Instrument Based on Cathode Relays«. Leonardo Music Journal 6, 49-50.

Toyota (2004): "Trumpet Robot«. <http://www.toyota.co.jp/en/special/robot> (last accessed October 2007).

Waiswisz, M. (1985): »The Hands: A Set of Remote MIDI Controllers«. In: Proceedings of the 1985 International Computer Music Conference. San Francisco: International Computer Music Association, 313-318.

Weinberg, Gil (2001): »The Squeezables: Toward an Expressive and Interdependent Multi-player Musical Instrument«. Computer Music Journal 25(2), 37-45.

Weinberg, Gil (2005): »Interconnected Musical Networks - Toward a Theoretical Framework«. Computer Music Journal 29(2), 23-39.

Weinberg, Gil/Lackner, Tamara M./Jay, Jason (2000): »The Musical Fireflies Learning About Mathematical Patterns in Music Through Expression and Play«. In: Proceedings of the 2000 Colloquium on Musical Informatics. LAquila, Italy: Instituto GRAMMA, 146-149.

Weinberg Gil/Aimi, Roberto/Jennings, Kevin (2002): »The Beatbug network: a rhythmic system for interdependent group collaboration«. In: Eoin Brazil (Ed.), New Interfaces for Musical Expression, NIME-02, Proceedings, Dublin: Media Lab Europe, 1-6.

Weinberg, Gil/Driscoll, Scott (2005): »iltur : Connecting Novices and Experts Through Collaborative Improvisation«. In: Sidney Fels/Tina Blaine (Eds.), New Interfaces for Musical Expression, NIME-05, Proceedings, Vancouver: University of British Columbia, Media and Graphics Interdisciplinary Center (MAGIC), 17-22.

\section{4}




\section{Acknowledgements}

The projects described in this paper would not have been possible without the valuable contribution of colleagues and students at MIT Media Lab and Georgia Tech Music department. In particular, I would like to thank Seum Lim Gan, Roberto Aimi, Tamara Lackner, Jason Jay, Scott Driscoll, Travis Thatcher, Mark Godfrey, Alex Rae, and John Rhoads for their indispensable contribution for the development of the musical instruments and applications. I would also like to thank Tod Machover, director of the Hyperinstrument group at MIT Media Lab, and Frank Clark, director of the Music Department at Georgia Tech for their support. 


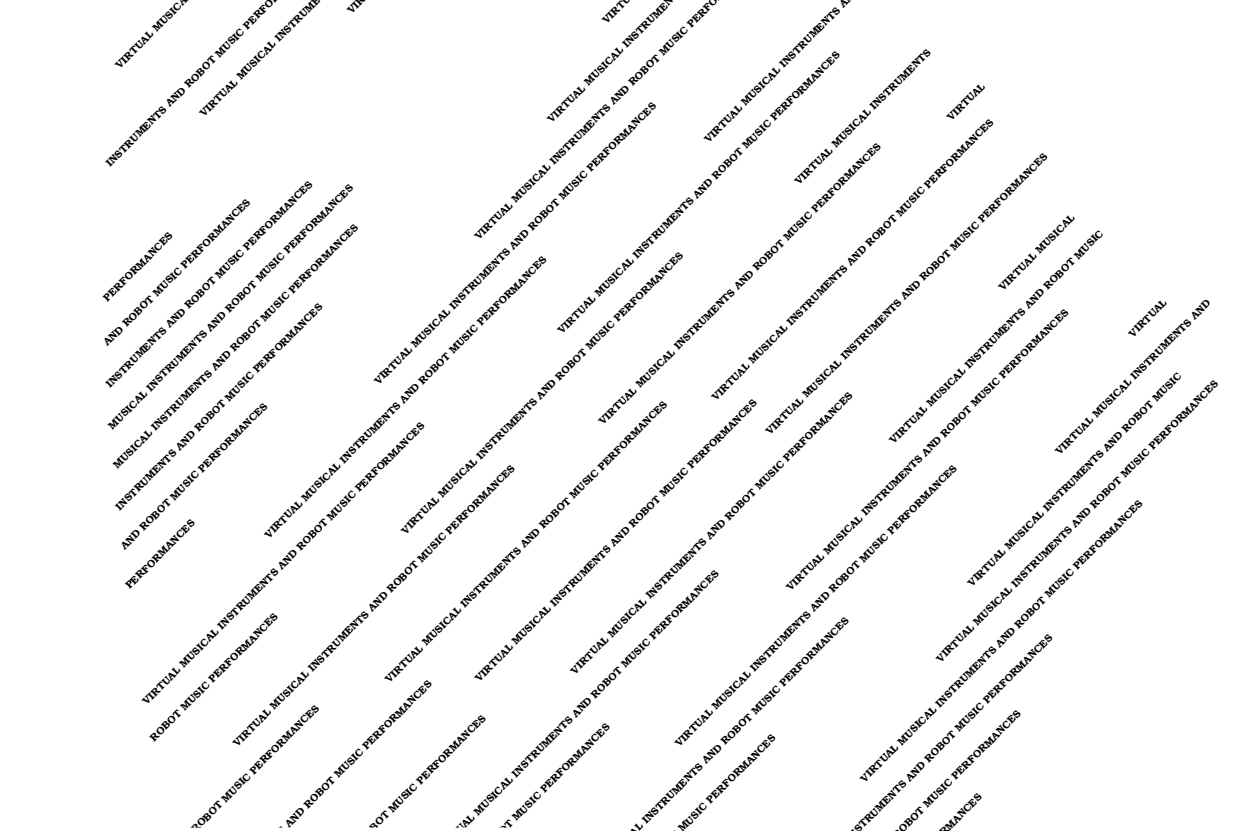

\section{VIRTUAL}

MUSICAL

INSTRUMENTS

AND

ROBOT MUSIC

PERFOR-

MANCES

Suguru Goto

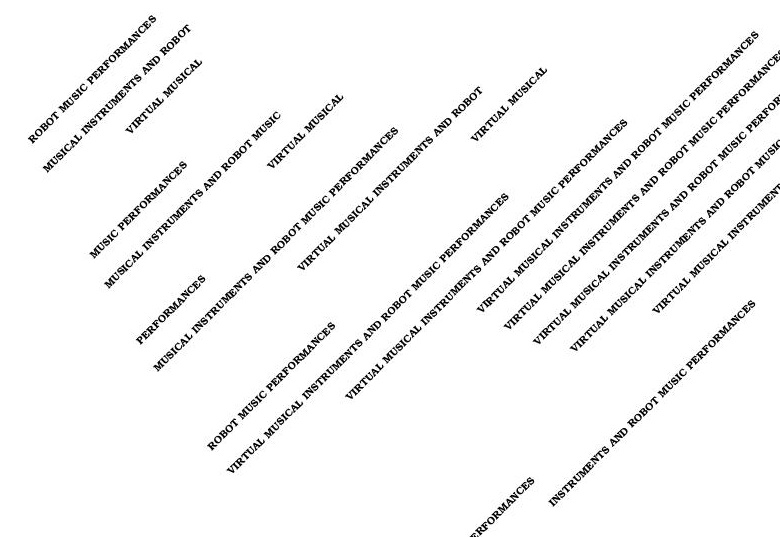




\section{Introduction}

The rapid evolution of the computer in the 90s introduced further developments in Artificial Intelligence, Artificial Life, and Virtual Reality, not to mention advances in multimedia and internet technology which are now an essential part of our lives. The people who have applied these technologies, are not only researchers, but also artists whose work could potentially change the way art is viewed. During the interplay of art and digital technology, many new amalgamated fields in the world of art have arisen: Interactive Music, Interactive Installations combining sound and imagery, Performance Art, Interactive Imagery etc.

Since the 1990s, I experimented with numerous compositions and performances using new technologies. Besides compositional concerns, my work has been based on questions of whether we are able to further develop the aesthetics that have been built by various artists in these different fields using interactive computer technology, and to create a new field of which the robot is the point of contact.

This paper is intended to cover my recent works: the virtual violin "SuperPolm" (1996), the "BodySuit" (since 1997), "RoboticMusic" (2003) and the project "Augmented Body and Virtual Body" (since 2002), both from the technical and aesthetic points of view.

\section{2. "SuperPolm"}

The Virtual Violin "SuperPolm" which was created with the collaboration of Patrice Pierrot and Alain Terrier in IRCAM, France in 1996, is one of the Virtual Musical Instruments that I created. Virtual Musical Instruments, which are defined as systems containing gesture, gesture interface, mapping interface, algorithm, and sound synthesis, consist of a gesture interface or controller, which cannot produce sounds by itself (Fig. 1). ${ }^{1}$

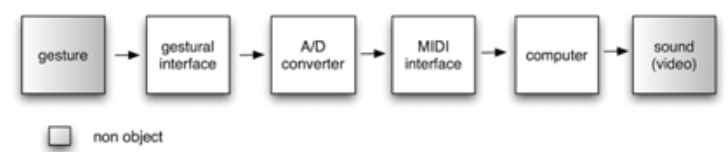

Fig. 1. Components of Virtual Musical Instruments

1 Goto 2000, p. 220 
It merely sends signals that produce sounds by means of a computer or a sound module. It may be regarded as an interface between the performer and the computer insofar as it translates the energy derived from body movements into electrical signals. At the same time however, it allows the performer to express complex musical ideas. With the capabilities of gestural interfaces of a controller, which can be modified by programming, a tiny gesture can trigger any number of complex musical passages at one and the same time in a real time context, whereas a traditional instrument can produce only a limited range of sounds. I use this principle of gesture interfaces as an essential factor in my compositions. One of my gestures at one moment might produce a sound similar to a traditional instrument but in the following section the same gesture might trigger a very different sound. As well as allowing for more possibilities in terms of sound, it also allows for a certain theatricality in performance. A controller is adapted to a specific type of gesture. In this case a controller refers to the gestural interface, but it also means a remote control device for manipulating a computer from a distance through MIDI (Musical Instrument Digital Interface), OSC (Open Sound Control) etc. ${ }^{2}$

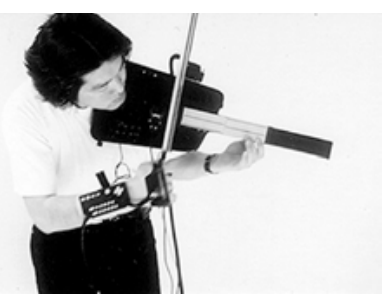

Fig. 2. The Virtual Violin, the "SuperPolm," is based upon the idea of short-range motion capture, such as finger, hand and arm movements.

The basic idea behind the SuperPolm is to interface gestures that resemble the playing of a musical instrument - in this case a violin - in order to control sound or images. These gestures are translated into parameters of position, pressure or distance by sensors based on the idea of short-range motion capture, such as finger, hand and arm movements. The resulting voltage is converted by an analogue-to-digital interface into MIDI signals that can be fed into a computer. The computer controls or generates the sounds in real time and can modify these signals by means of algorithms. For example, a single channel signal can be altered to become a rich and complex sound such as that of an orchestra (Fig. 2). The SuperPolm contains a force sensor placed in the chin rest and an inclinometer measuring respectively the performer's constraint to maintain the instrument and the angle impressed towards the vertical. Therefore the performer can control two added parameters without hand movements using chin pressure and/or bending the upper body forwards.

The SuperPolm can be played in a similar manner to the violin, except that the fingers touch sensors on a fingerboard instead of pressing strings,

$2 \quad$ Goto 1999

\section{8}


since there is neither string nor hair of bow: A gesture of performance with a violin is merely modeled (Fig. 2). However, movements of the bow causing variations in resistance can, assign new functions as well as modify sounds. An eight-button keyboard situated on the body of the instrument can change both the program in Max/MSP/Jitter and the sounds, as well as triggering different pitches, like a normal keyboard. Hence, new functions of programming can be taken into account according to the compositional needs of each piece: for instance a sensor can be used to trigger sounds in one composition, whereas in another it can be used to change the pitch.

The SuperPolm was originally intended for use in a piece I composed at IRCAM in 1995-1996, entitled "VirtualAERI". The first performance of this piece was given in 1997 at IRCAM's Espace de Projection. It consisted of four sections, each of which dealt with a different kind of space, large, medium and small. The SuperPolm was designed for one particular section of this composition focusing specifically on the possibilities opened up by the controller.

The SuperPolm can control not only the parameters of sound synthesis, but also those of images in real time (Fig. 3). For instance, it can superimpose live or sampled images on top of each other, add effects, such as delay, and speed up, reverse or repeat these images. It can also mix several images in different proportions and modify their colour, brightness and distortion, while the sampled images can be started and stopped at any point.

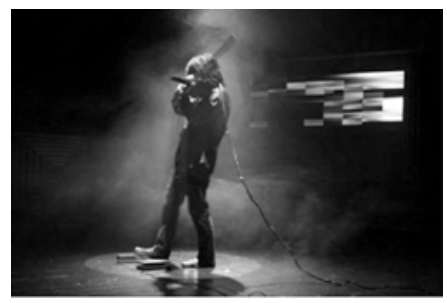

Fig. 3. The SuperPolm can also control the parameters of images in interactive videos (Photo and Copyright: Arianna D'Angelica).

\section{3. "BodySuit"}

In my projects, Virtual Musical Instruments have been used in a performance context. Another instrument I have designed is the "BodySuit", a suit fitted with bending sensors that are attached to each joint of the body. It was built between 1997 and 1999 with the aim of a motion capture for the entire body, so that a performer wearing it can merely produce sounds with his gestures by bending and stretching each joint, without controlling any instruments in his hands.

The "BodySuit" is equipped with 12 sensors which are attached on each joint of the body. Depending on a movement, sound and video images are changed in real time. This differs from a traditional instrument or an instrument-inspired controller. A player performs with larger movements, such as 
stretching and bending joints, twisting arms and so on. This gesture does not function like dance or theater. It contains, however, an element of "performance" within the live, musical context. The gesture is not previously decided in

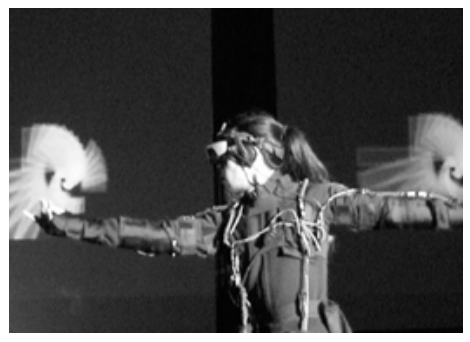

Fig. 4. The BodySuit enables to make wide, sweeping movements that can easily be observed by the audience. Performance at the University of Cologne in April 2004 (Photo: Franca Lohmann)

a strict sense. An audience may observe an obvious difference of intensity of movement between a static section and a kinetic section in the composition (Fig. 4). This suit is therefore an ideal tool in a musical theater situation.

Although the performer's gesture does not resemble those used for playing a musical instrument, I used the BodySuit exclusively as a virtual musical instrument. In particular, this works efficiently with percussionist-like gestures, as designed for my project "RoboticMusic" which will be described in section 3 in detail.

\section{4. "RoboticMusic"}

The act of performing music is not only about the control of a complicated set of body movements. On the one hand, music can be seen as a logical sequence of events over time which occur as a result of problem solving and rely on the interaction of a set of parameters. On the other hand, music derives itself from less calculable things such as 'good' rhythm sense and poetic significance and expression.

Taking into account such aspects of music performance, I was interested in the question of whether it would be possible for a robot making music to think logically, to play with emotion, to have a good sense of rhythm, to realise poetic expression, to achieve proper pitch (frequency) and delivery of sound, and to have a sense of proportion through comparison. Therefore I have been engaged in projects using robots in music performances, especially robots playing musical instruments.

My project "RoboticMusic" was designed for robots that consist of Snare Drum, Bass Drum, Cymbal, Gong, and Pipe. These have a specially designed springs to imitate human muscles. Each holds a mallet at the end of its arm (Fig. 5). One of the robots plays numerous pipes, and rapidly spins to create Flute-like sounds, which are generated as the air goes through them. These pipes are different lengths according to the pitches one desires. As it spins faster, the pitches become higher, moving up the overtone series. These 
mechanisms are created by me with technical help from Fuminori Yamazaki, iXs Research Corporation in 2003.

These robots performing on musical instruments are connected by computer and controlled with a program. Max from Cycling'74 is utilised both as an interface and to generate musical data. With this, one can also send basic parameters to the robots such as a position of the robot's arm, an offset position, intensity (how hard it hits) and so on. This sends the signals to another computer running Linux via UDP (Universal Data Protocol). This software in Linux is developed by iXs Research Corporation. This plays an important role since it controls the robot's movement. The Linux computer and robots are connected via USB (Universal Serial Bus). Each robot has its own interface which is connected with an actuator and a sensor.

The major advantage of "RoboticMusic" is that it interactively plays an acoustic instrument with the aide of a computer. There is no problem with playing complex rhythms which easily outperform human capabilities. Therefore, it gives new potentialities in composition for acoustic instruments. While a computer generated sound has many capabilities, an acoustic instru-

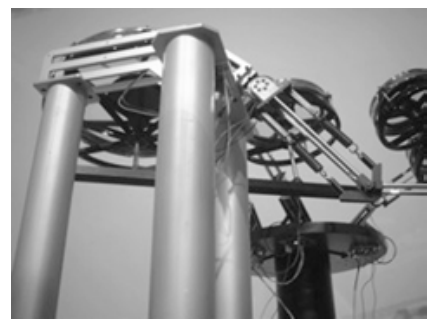

Fig. 5. Each holds a mallet at the end of his arm and can play any instruments as long as these can be played with Mallets. ment has rich sonority and enormous pos-

sibilities of expression, especially from the composer's point of view. With staged peformance the vast possibilities of the acoustic aspect are obvious when compared to sound coming from speakers. Another benefit is that the audience may observe both the source of the sound and the accompanying gestures necessary for its production.

I explored some musical compositions in order to see what only these robots could play. For instance, five robots in the project "RoboticMusic" played on musical instruments with different tempos at the same time, or intricate accelerando and ralentando, and yet synchronisation was maintained during the playing process. Each algorithm is assigned to each robot, but these 5 algorithms are controlled by one central program.

Other possibilities allow the robots to improvise and compose by themselves in real time during their performances with the aide of the computer's algorithm. During these performances, they sometimes played with computer-generated sounds at the same time. While I played a laptop, the robots accompanied me. In these performances, the lights were much emphasised, as well. As the robots changed their performances, the automated lights communicated with them via MIDI. The music, gestures on stage, and the 


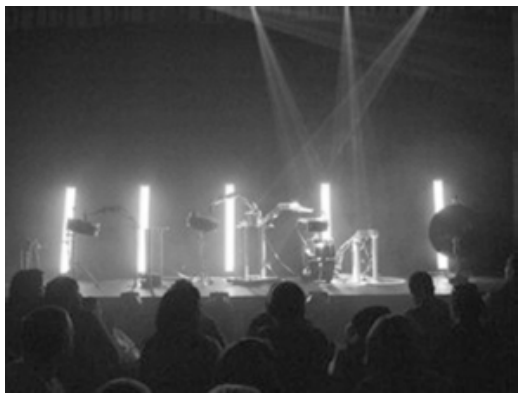

Fig. 6. Especially, these robots can show a lot of potentialities in concerts. The robots can certainly perform faster and more accurately than human players.

visual elements of the lights were integrated into one whole stage performance in RoboticMusic (Fig. 6).

\section{5. "Augmented Body and Virtual Body"}

This project "Augmented Body and Virtual Body" originally started in 2002. The system used for this project consists of the Data Suit, "BodySuit", and the Percussion Robots, "RoboticMusic", controlled in real-time by gestures produced with "BodySuit". This system

was intensively experimented with and shown on several occasions during 2005. The last performance was realised in, "Le Cube," in France in April 2006. The idea behind the system is that a human body is augmented by electronic signals in order to be able to perform musical instruments interactively. A gesture of a performer with "BodySuit" is translated to gestures of "RoboticMusic." One of the important elements is the relationship and the communication method explored within this system. One may consider "BodySuit" and "RoboticMusic" as a relationship between a conductor and an orchestra, where dance-like gestures trigger instruments. In other words, this is an instrument that relies on physical gestures. Another point is the method of translation used by the computer. For example, signals from "BodySuit" are transformed by mapping interface and algorithm in a computer, and routing them into "RoboticMusic." One gesture may trigger one attack on one instrument. However, it is also possible to trigger five instruments at the same time. Otherwise complex, musical data, which is automatically generated by a computer and then reproduced by "RoboticMusic", is altered with gestures from "BodySuit" to modify the parameters of an algorithm in real time. ${ }^{3}$

The robots' reactions to the "BodySuit" performer's gestures can be either direct or indirect. For example, a rapid arm gesture from a higher position to lower could trigger the percussion robot to hit an instrument, or a gesture triggers an algorithm of particular behaviour that sends signals with various values of delay to each robot. The robots which are controlled by the computer, only play in certain sections of the composition. While the passages which are controlled by "BodySuit" make it possible to create a more complex musi-

3

Goto 2006

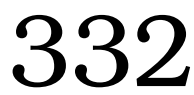


cal material. The robot solo with the computer allows realisation of complex, high speed performance, which is impossible for human instrumentalists.

While the communications of gesture with "BodySuit" and "RoboticMusic" are observed, one notices different phases of interaction, which are the interaction with its perception, and the interaction with its consciousness. These two poles are important keys in this field. With the articulated visual and oral experience in this work, one may recognise different experiences that constantly deal with something to expect, to understand, to notice, and to perceive. Furthermore, the relationship between gesture and sound is also seen differently with this system. In other words, the idea of, "music to see, visibility to hear" brings a different context into theatrical performance.

Let us see the relationship between "BodySuit" and "RoboticMusic". The first is designed to control others with the will of a performer. The latter is designed to be controlled by someone else. However, both of these equate to bodies that are extended with the medium of electronic devices. The meaning of body, which can exist as being virtual or being augmented, are intentionally mixed. On the other hand, when we see the relation-

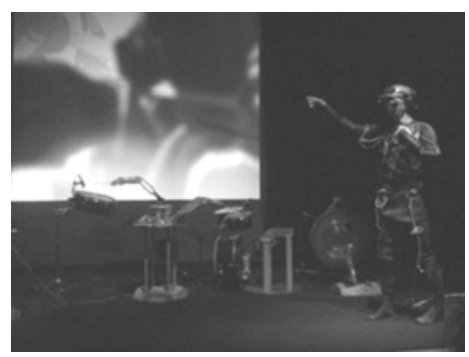

Fig. 7. The BodySuit works well with percussionist-like gestures. This is one of the best controller conjunctions with "RoboticMusic".

ship between "BodySuit" and "RoboticMusic", this appears as a relationship between the physical world and the virtual world. While the robots consist of artificial bodies compared with human bodies, they could also be physical bodies contrasted with virtual bodies.

\section{Conclusion}

The idea underlying my projects sketched above is to explore the dualism of the real and the virtual and the relationship between artificiality and reality of the human body. Artificiality and reality sometimes seem to be in conflict with each other, but they can work together, or their meaning can be transformed for an audience depending on the context. The context provokes the audience into playing with the ideas of reality and artificiality. A performance involving "BodySuit", and "RoboticMusic" challenges the audience by confusing the line between virtuality and reality. As a composer I intend to create such a composition which emphasises the importance of performance aspects with this system. 
While the concept of "Extended Body" was conceived to be realised with these systems mentioned above, the theme "Augmented Body and Virtual Body" is meant to question what a human body is and what its own identity is with this. Man and Machine seem to be dualistic, more precisely, one may think that they are conflicted against each other. In my projects, however, they coexist within an interactive, artistic system: man and machine are regarded as being one - an "Extended Body". As a result, our identity is not merely confined within the boundaries set up by our body, but becomes extended. 


\section{References}

Goto, Suguru (2000): „Virtual Musical Instruments: Technological Aspects and Interactive Performance Issues«. In: Marcelo M. Wanderley/Marc Battier (Eds.), Trends in Gestural Control of Music, Paris: IRCAM, 217-230.

Goto, Suguru (1999): »The Aesthetics and Technological Aspects of Virtual Musical Instruments: The Case of the SuperPolm MIDI Violin«. Leonardo Music Journal 9, 115-120.

Goto, Suguru (2006): »The Case Study of An Application of The System, "BodySuit" and "RoboticMusic": Its Introduction and Aesthetics«. In: Norbert Schnell/Frédéric Bevilacqua/Michael J. Lyons/Atau Tanaka (Eds.), New Interfaces for Musical Expression, NIME-06, Proceedings, Paris: IRCAM - Centre Pompidou in collaboration with Sorbonne University, 292-295.

Augmented Body and Virtual Body: <http: / 0141712186.free.fr/Contents2/ABVB-E/ AugmentedBodyAndVirtualBody-e.html>; <http://0141712186.free.fr/Contents2/ LeCube/LeCube-e.html> (last access: July 2008).

BodySuit: <http://suguru.goto.free.fr/Contents/Works /BodySuit/BodySuit-e.html> (last access: July 2008).

RoboticMusic:<http: / / suguru.goto.free.fr/Contents/Works/RoboticMusic/RoboticMusic-e .html>; <http: / / 0141712186.free.fr/Contents2 / AVFestival/AVFestival-e.html > (last access: July 2008). 


\section{Authors' Biographies}

Antonio Camurri (born in Genoa in 1959; 1984 Master's Degree in Electric Engineering; $1991 \mathrm{PhD}$ in Computer Engineering) is Associate Professor at the DIST University of Genoa (Faculty of Engineering), where he teaches Software Engineering and Multimedia Systems. His research interests include computer music, multimodal intelligent interfaces, interactive systems, kansei information processing and artificial emotions, interactive multimodal-multimedia systems for theatre, music, dance, and museums. He is founder and Scientific Director of the InfoMus Lab at the DIST University of Genoa (www.infomus.org). He was President of AIMI (Italian Association for Musical Informatics), is member of the Executive Committee (ExCom) of the IEEE CS Technical Committee on Computer Generated Music, Associate Editor of the international "Journal of New Music Research". He is in charge of EU IST Projects at DIST InfoMus Lab of the University of Genoa. He is the author of more than 80 international scientific publications. Since 2005 he has been Director of the Casa Paganini International Centre of Excellence for science and multimedia technologies for music and performing arts (www. casapaganini.org).

Hans H. Diebner has been head of the project "Basic Research into Performative Science" at the Institute for New Media, Frankfurt am Main since January 2006. He studied Physics in Tübingen, graduated in 1994 with a Diploma Thesis on exactly reversible molecular dynamics simulations and received his PhD in 1999 with a Doctoral Thesis on "Time-dependent deterministic entropies and dissipative structures in exactly-reversible Newtonian molecular-dynamics universes." Diebner's research is a continuation of endophysics, a notion coined by his supervisor, the eminent chaos researcher Otto E. Rössler. The annulment of the object-subject distinction in endophysics is emphasised in performative science by adding corporeality to the methodological canon of science. From 1999 until 2005 Diebner headed the Institute for Basic Research at the Centre of Art and Media in Karlsruhe, where he worked on the concept of performative science and collaborated in numerous projects with artists as well as scientists from different disciplines. He feels at home both in general system theory and in the arts. The achievements of the ZKM period have been published in 2006 by Springer-Verlag Vienna, entitled "Performative Science and Beyond - Involving the Process in Research." Besides his scientific practice he is deeply committed in education, and endeavours to establish performative science as an interdisciplinary area of studies.

\section{6}


Monika Fleischmann is a German media artist and scientist and Hon. Professor for Media Art and Theory of the University of Applied Sciences, Bremen. Since 1997 she has been head of Media Arts \& Research Studies at the MARS - Exploratory Media Lab at the Fraunhofer Institute IAIS. Her main research topic is to extend the concept of communication through interaction and participation on the base of perceptive processes. She has published more than 100 scientific papers, artistic essays and theoretical articles. In 1987 she co-founded Art+Com in Berlin with artists, architects and computer scientists. Since then she has produced a large number of media art and design objects in cooperation with her partner Wolfgang Strauss. In co-operation with her partner she has received 22 awards (June 2008). Her important works of art include "Home of the Brain" (1992) (first artistic HMD - VR Installation), "Liquid Views” (1992-93) - Narcissus' mirror and "netzspannung.org - performing the archive" (2006).

Stefan Göllner studied Landscape Architecture at the Technical University Berlin (1997-2000) and Communications Design at the Düsseldorf University of Applied Sciences (2000-2006). Since February 2007 he has been working at the Academy of Media Arts Cologne (KHM) in the EU-funded research project "Citizen Media". His focus in the project is on civic media in local communities and the development of a map-based platform for participatory urban development ("Unortkataster Köln”).

Suguru Goto is a composer/performer, an inventor and a multimedia artist, and he is considered one of the most innovative of and the mouthpiece of a new generation of Japanese artists. He is highly connected to technical experimentation in the artistic field and to the extension of the existing potentialities in the man-machine relation. In his works the new technologies mix in interactive installations and experimental performances, he is the one who invented the so-called virtual music instruments, able to create an interface for the communication between human movements and the computer, where sound and video image are controlled by virtual music instruments in realtime through computers. Lately, he has been creating robots which perform acoustic instruments, and he is gradually constructing a robot orchestra. $\mathrm{He}$ has been internationally active and has received numerous prizes and fellowships, such as the Koussevitzky Prize, BSO fellowships, the first prize at the Marzena, Berliner Kompositionaufträge, a prize from the IMC International Rostrum of Composers in the UNESCO, Paris, DIRECAM, the French Cultural Ministry, and so on.

http: / / suguru.goto.free.fr / 
Ludwig Jäger has held a professorship in linguistics and communications studies at the RWTH Aachen since 1982. He is at present spokesman of the Collaborative Research Centre SFB/FK 427 "Media and Cultural Communication" of the Universities of Aachen, Bonn, and Cologne. He has been a member of the Review Panel of the Swiss National Science Foundation, the "Cercle Ferdinand de Saussure", and the "Société de Linguistique de Paris" among others. He is co-editor of the journal "Sprache und Literatur" and editor of the series "Mediologie" in DoMont press (henceforth Fink). His main research issues include media theory, sign theory, and the history of linguistic theories. Recent publications: Medienbewegungen. Praktiken der Bezugnahme. München: Fink (co-ed. with Gisela Fehrmann and Meike Adam, in print); Deixis und Evidenz. Freiburg i.Br.: Rombach (co-ed. with Horst Wenzel, in print).

Rudolf Kaehr was born in Switzerland and studied Philosophy, Psychology, Linguistics, Logic, and Mathematics at the universities of Zürich, Münster (Westfalen) and the Free University of Berlin and earned a Dr. phil. (PhD) - summa cum laude - with the philosopher and researcher of cybernetics Prof. Gotthard Günther (BCL, Ill., USA) at the University of Hamburg. He was Director of the Institute of Theoretical Biosciences at the Private University Witten/Herdecke (1986 to 1990), has been lecturer at international universities and academies of arts since 1971, and was Visiting Professor at the Academy of Fine Arts, Philosophy, Frankfurt/M., Germany (1998 to 2000), Research Associate at the Academy of Media Arts, Computer Sciences, Cologne (1999 to 2002) and Research Associate at the Goldsmiths College, Centre for Cultural Studies, University of London, 2004-2005. He is at present Director of the ThinkArt Lab Glasgow (http: / www.thinkartlab. com) in Scotland, where he lives. He is a founding member of the Institute for Cybernetics and Systems Theory e.V. Bochum, Germany (1991).

Jin Hyun Kim studied Musicology and Philosophy at the Seoul National University (Korea) and at the University of Hamburg and received her $\mathrm{PhD}-$ summa cum laude - at the University of Osnabrück, with a Doctoral Thesis on embodiment in interactive music and media performances - taking into account perspectives from media theory and cognitive science. Since 2002 she has been a member of the Collaborative Research Centre SFB/FK 427 "Media and Cultural Communication" at the Universities of Aachen, Bonn, and Cologne. Within the scope of this, she is currently engaged in the research project "Artistic Interactivity in Hybrid Networks" dealing with interactive audio programming (live coding), robotic arts and Artificial Life Art in cooperation with the Academy of Media Arts Cologne. Her current research issues include New Media Theory, embodiment and mediality of (musical) aisthesis

\section{8}


and (music) cognition, interdisciplinary approaches to musical expressiveness, interactive music and media performances. She has published articles on media theory, media art, music aesthetics, music cognition, interactive music, sound computing, and human-computer and human-robot interaction, among other things.

Sybille Krämer is Professor of Philosophy at the FU Berlin and a permanent fellow at Wissenschaftskolleg Berlin. She is a founding member of the Helmholtz-Zentrum für Kulturtechnik and has been a member of the German Council of Science and Humanities from 2000 to 2006 and a referee of the European Resarch Council since 2007. She is supervisor of both the project as part of the Collaborative Research Centre "Kulturen des Performativen" and the interdisciplinary research group "Bild, Schrift, Zahl", and is spokewoman of the research training group 1458 "Notational iconicity: On the materiality, perceptibility and operativity of writing". Furthermore, she has conducted several projects in the field of philosophy of language and media theory funded by the German Research Foundation. Her main research isseus include philosophy and mathematics in the early modern age, theory of mind and of consciousness, interpretations of computers, semiotics and media theory, philosophy of language and basic problems of cultural studies. Selected recent publications: (ed.) Performativität und Medialität 2004; (co-ed. with Grube/Kogge) Schrift. Kulturtechnik zwischen Auge, Hand und Maschine 2005; (co-ed. with Doris Kolesch) Stimme 2006; (co-ed. with Grube/ Kogge) Spur. Spurenlesen als Orientierungstechnik und Wissenskunst 2007; Medium, Bote, Übertragung. Kleine Metaphysik der Medialität 2008.

Martina Leeker is at present a Junior Professor of Theatre and Media at the Universität Bayreuth. She studied Theory of Theatre, Philosophy and German Studies in Berlin and Paris, and finished her education in theatre in Paris under Etienne Decroux and Jacques Lecoq. Combination of theory and practice in "Theorietheater" is a part of the profile of her work. The main focuses of her research are theatre and media (intermediality), the history and theory of computers, the combination of science, media and art, theatrality and performativity of Web 2.0. Her most important publications are Mime, Mimesis und Technologie, München 1995; Maschinen, Medien, Performances. Theater an der Schnittstelle zu digitalen Welten [with CD-ROM "Interfaces, Interaktion, Performance" of Irina Kaldrack and Martina Leeker], Berlin 2001; (with Söke Dinkla as coauthor) Tanz und Technologie. Auf dem Wege zu digitalen Inszenierungen [with DVD], Berlin 2002; and (co-ed. with Derrick de Kerckhove and Kerstin Schmidt) McLuhan neu lesen. Kritische Analysen zu Medien und Kultur im 21. Jahrhundert, Bielefeld 2008 [with DVD]. She has also written various papers about theatre/performance and 
media, about the history of theatre of computers, and about the history of computer of theatre.

Christoph Lischka studied Composition, Piano, Musicology, Philosophy, and Mathematics at Cologne University of Music, the University of Cologne, and the University of Bonn. He has worked as a software engineer, artist, research scientist, and university lecturer at several institutions (e.g. the Fraunhofer Institute St. Augustin, Cologne University of Music, University of Cologne, and the Academy of the Arts Düsseldorf) in the fields of Artificial Intelligence, Music Theory, Cognitive Science, Philosophy, and Robotics. In his current research the focus is put on the interplay of art and convergent technologies (NBIC), particularly nanobiotechnology. Since 2007, Christoph Lischka has been Professor of Poietic Machines (Autoaktive Systeme) at the University of the Arts Bremen.

Barbara Mazzarino, $\mathrm{PhD}$, computer engineer, is research assistant at University of Genova, Casa Paganini - InfoMus Lab. Her research interests include real-time analysis of expressive content in human full-body movement and gesture, multimodal interfaces, multimodal interactive systems for performing arts and for therapy and rehabilitation. She is currently working as local project manager at the EU-ICT Coordination Action CAPSIL on ambient assisted living and remote monitoring of elderly and disabled people.

Anthony Moore is a composer and media artist and, since 1996, tenured Professor at the Academy of Arts and Media Cologne in the department of Art and Media Science, where he works on the theory and history of sound. $\mathrm{He}$ is the author of numerous musical pieces, songs, sound installations and film compositions which have received international prizes; in 1972 he was founder of the band "Slapp Happy"; he was co-writer with the music group "Pink Floyd" during the recording of two albums, working on concepts, sounds and lyrics. He composed the television opera "Camera", a commissioned work from Channel 4. Polygram recorded three albums of his work, "Pieces from the Cloudland Ballroom", "Secrets of the Blue Bag" and "Reed, Whistle \& Sticks", for voices, strings, woodwind and percussion. In 1996 he became Professor for Auditive Design in the media and head of the Music Department at the Academy of Arts and the Media Cologne (KHM); from 2000 to 2004 he was elected Principal of the Academy in Cologne. He has been the initiator and art director of a number of sound events, including "per $>$ SON". Besides lecturing he continues to write and compose.

\section{0}


Frieder Nake is a mathematician who is also recognised, through national and international exhibitions, as an artist. However, for decades his professional career has been in computer science, specialising in computer graphics and interaction. He has held positions at the universities of Stuttgart, Toronto, British Columbia (Vancouver), and Bremen. Since 1972 he has developed a critique and theory of computing which led him into the design of digital media. His concepts are machinisation of mental labor, instrumental medium, semiotic machine, and algorithmic sign. Since 2004, he has been with the University of the Arts in Bremen. Selected publications: Ästhetik als Informationsverarbeitung, Vienna/New York 1974; Die erträgliche Leichtigkeit der Zeichen. Ästhetik, Semiotik, Informatik, Agis, BadenBaden 1994; (with Diethelm Stoller) Algorithmus und Kunst. Die präzisen Vergnügen, Hamburg, 1993.

Werner Rammert is Professor of Sociology and Social Studies of Technology and Director of the Centre for Technology and Society at the Berlin Institute of Technology. He was the co-founder of the yearbooks "Technology and Society" (10 vol.) and of the interdisciplinary DFG program "Socionics" (6 years). He has been a visiting professor/fellow at the Vienna Institute of Technology, at Stanford University, and at the Advanced Centrer $\mathrm{ZiF}$ at the University of Bielefeld. His research interests include philosophy and the history of technology, the social theory of pragmatism, technology and innovation studies, human-technology relations, and media studies (telephone, PC, knowledgebased systems, ubiquitous computing). His TUTS research group develops the tools and methods of "socio-technical constellations analysis", "technography", and "innovation biography".

Julian Rohrhuber currently works in the research project "Artistic Interactivity in Hybrid Networks" at the University Cologne and the Academy of Media Arts. Investigating the culture of abstraction and foundational discourses, he works on algorithmic acoustics, art theory and philosophy of science. Specific projects include: a system for interactive programming, sonification in science, algorithms for cultural anthropology. His interests also include video and sound installations and film sound. Select recent publications: New Mathematics and the Subject of the Variable (in: Variantologie 4 in print); Network Music (in: The Cambridge Companion to Electronic Music 2008); Mengenlehre (in: Unmenge - Szenen verteiler Handlungsmacht 2008); Artificial, Natural, Historical. Acoustic Ambiguities in Documentary Film (in: Transdisciplinary Digital Art: Sound, Vision and the New Screen, Communications in Computer and Communication Science 2008); (with Renate Wieser) The Invisible Hand (in: Readme 100. Temporary Software Art Factory 2007). 
Lasse Scherffig studied Cognitive Science at the universities of Osnabrück/Germany and Oswego/USA and Digital Media at the University of Bremen/Germany and the Academy of Art and Design Zürich/Switzerland. His master thesis was written during a scientific residence at the Insitute for Basic Research of the ZKM, Karlsruhe. Since 2006 he has been a member of the artistic/scientific staff of the Academy of Media Arts Cologne working in the fields of experimental computer science and human-computer interaction.

Uwe Seifert studied Musicology, Computer Science and Philosophy at the University of Hamburg, from which he received the Magister Artium, the Promotion (PhD) and the Venia Legendi or Habilitation (postdoctoral qualification) in Systematic Musicology. Since 1999 he has held a professorship in Systematic Musicology at the University of Cologne. He has been a member of the Collaborative Research Centre SFB/FK 427 "Media and Cultural Communication" since 2002, in which he has been leader of the research projects "Electronic Music Transformation since 1950 - Transcriptive Interaction" (2002-2004) and "Artistic Interactivity in Hybrid Networks" (since 2005). Since 2006 he has been partner of the International Summer School in Systematic Musicology funded by the European Union. His current research interests include New Media Theory, New Media Art, (embodied) cognitive science of music, cognitive musicology, and methodological problems in music research.

Wolfgang Strauss is a German architect, media artist and scientist. He studied Architecture at the Hochschule der Künste in Berlin and has been head of the MARS - Exploratory Media Labs R\&D since 1997 for interactive and mixed reality environments. He creates real buildings as well as electronic architecture, knowledge spaces and digital archives. He has had guest professorships and fellowships, and has given talks internationally. Since 1987, when he co-founded Art+Com in Berlin, he has produced a large number of media art and design objects and environments on the topics of networked information space and the visualisation of knowledge in cooperation with Monika Fleischmann. In co-operation with his partner he has received 22 awards (June 2008). He has published more than 100 scientific papers, artistic essays and theoretical articles. Recent R\&D activities have been knowledge discovery tools, artistic installations such as "Energie-Passagen", applications such as the "interactive poster" and patented interfaces like the contactless "PointScreen". http: / / netzspannung.org/about/mars/projects/en

\section{2}


Georg Trogemann has been a full Professor for Experimental Computer Science at the Academy of Media Arts Cologne since 1994. He studied Information Technology and completed his doctoral degree in 1990. His research focuses on pattern recognition and mathematics. He is a member of the scientific staff at the University in Erlangen. He received the 1993 Prix Ars Electronica, Linz, as well as the Golden Nica Award for Interactive Art with the Knowbotic Research Group. He had a study residence in England in 1993 and was a guest at the Royal College of Art, London. His research interests include Art und Design Research, Interface Cultures, and the Theory of Artefacts.

Gualtiero Volpe, $\mathrm{PhD}$, computer engineer, is assistant Professor at University of Genova. His research interests include intelligent and affective human-machine interaction, modeling and real-time analysis and synthesis of expressive content in music and dance, and multimodal interactive systems. He is member of the Board of Directors of AIMI (Italian Association for Musical Informatics). He was Chairman of the V Intl Gesture Workshop and of the 8th Intl Conference on New Interfaces for Musical Expression (NIME 2008), and Guest Editor of a special issue of Journal of New Music Research on "Expressive Gesture in Performing Arts and New Media" in 2005.

Gil Weinberg is the Director of Music Technology at Georgia Institute of Technology, where he founded the Master of Science in Music Technology program and the cross-campus Music Technology Research Center. In his academic work Weinberg attempts to expand musical expression, creativity, and learning through meaningful applications of technology. His research interests include new instruments for musical expression, musical networks, machine and robotic musicianship, sonification, and music education. Weinberg's music has been featured in festivals and concerts such as Ars Electronica, SIGGRAPH, ICMC, and NIME, and with orchestras such as Deutsches Symphonie-Orchester Berlin, the National Irish Symphony Orchestra, and the Scottish BBC Symphony. He has published more than 30 peer-reviewed papers. His interactive musical installations have been presented in museums such as the Smithsonian Museum, Cooper-Hewitt Museum, and Boston Children's Museum. With his perceptual robotic percussionist, Haile, he has traveled around the world, giving dozens of concerts. As a result of this project, Weinberg has recently been awarded a National Science Foundation grant to continue to explore the concepts of machine and robotic musicianship. He received his M.S. and Ph.D. degrees in Media Arts and Sciences from the Massachusetts Institute of Technology, after cofounding and holding a number of positions in the music and media software industry in his home country Israel. 
